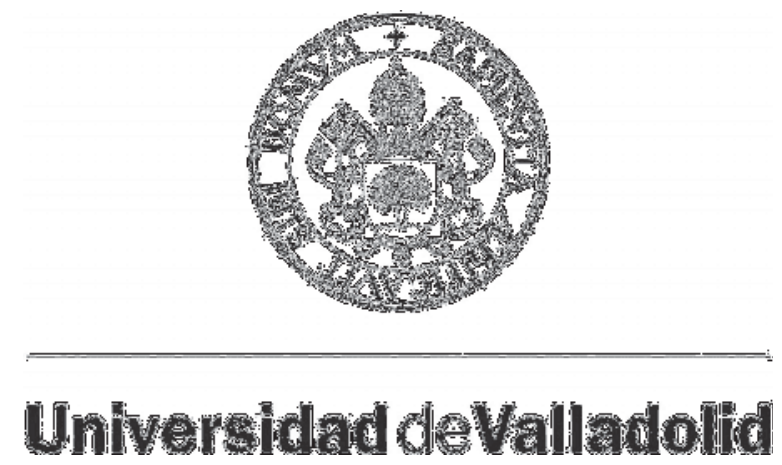

Facultad de Ciencias

Departamento de Física de la Materia Condensada,

Cristalografía y Mineralogía

TESIS DOCTORAL:

Transporte térmico y caracterización Raman de nanohilos semiconductores de Silicio y Silicio-Germanio

Presentada por Julián Anaya Calvo para optar al grado de doctor por la Universidad de Valladolid

Dirigida por:

Juan Jiménez López 



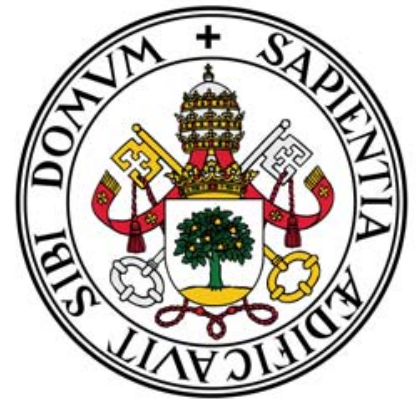

UNIVERSIDAD DE VALLADOLID

\title{
TRANSPORTE TÉRMICO Y \\ CARACTERIZACIÓN RAMAN DE \\ NANOHILOS SEMICONDUCTORES DE \\ SILICIO Y SILICIO-GERMANIO
}

\author{
Tesis doctoral presentada por Julián Anaya Calvo \\ dentro del Programa de Doctorado en ciencias Físicas \\ Dirigida por el Prof. Juan Jiménez López \\ El doctorando \\ El director
}

Valladolid, agosto 2013 

Transporte térmico y caracterización Raman de nanohilos semicondutores de Silicio y Silicio-Germanio

Autor: Julián Anaya Calvo

Director: Juan Jiménez López

Manuscrito impreso en Valladolid

Primera edición, agosto 2013 

A mis padres, sin vuestro apoyo y paciencia nunca habría llegado hasta aquí.

Pg9qp/I6shqjjbIUdjCUN8n47HxOaTDPgCDOE1 tipB8 8a3h/svGd66IBTAQ/IhQSOLhXObXhHha6sEo6/gER4 RtAoeukFmupIsPA8S4nHdZ/aER5VORi405Pb+RYY3tz

Julian Anaya

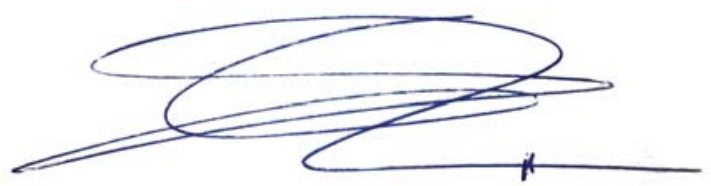





\section{Agradecimientos}

Primeramente me gustaría agradecer a mi director Juan Jiménez la oportunidad que me brindó hace algo más de tres años y que me ha permitido llegar hasta aquí. Gracias a sus consejos y supervisión constante he podido concluir la investigación desarrollada en esta tesis, pero también he podido aprender de él más física de la que jamás podría haber imaginado, siendo sin ninguna duda la persona que más ha influido en mi formación académica.

También me gustaría dar las gracias por toda la ayuda y soporte que me han dado durante estos años de investigación a mis colegas del grupo de investigación OptronLab de la Universidad de Valladolid. Vanesa, Benito, Oscar, Ángel Carmelo, Miguel Ángel, Alberto, Jorge etc... sin vuestro apoyo y ayuda no podría haber terminado este proyecto.

Mención especial merecen mis amigos Victor, Sergio, Javier, Daniel, Cristina, Ana, Soraya, etc... por distraerme y ayudarme en los momentos de desesperación acaecidos durante la investigación y confección de esta tesis

Por último, pero no por ello menos importante, debo agradecer a mis padres y a mi novia todo lo que me han ayudado durante estos años de investigación, ellos han sido los hombros sobre los que he llorado en mis momentos de desesperación y los que más han disfrutado con mis alegrías, esta tesis no podría haberse hecho sin vuestra ayuda, comprensión y cariño, gracias.

Julián Anaya

agosto 2013

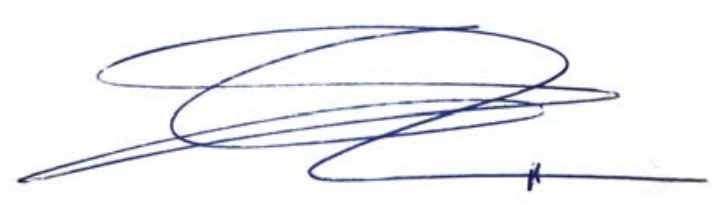





\section{Índice general}

Índice de figuras $\quad$ ix

Índice de cuadros $\quad$ xxxiii

1 Introducción 3

1.1 Nociones básicas sobre el scattering Raman . . . . . . . . . . . 4

1.1.1 Scattering Raman desde un punto de vista clásico . . . . . 5

1.1.2 Scattering Raman desde la interpretación de la mecánica cuántica . . . . . . . . . . . . . . . . 9 9

1.2 Descripción del equipo Raman . . . . . . . . . . . . . . . 11

1.3 Microscopio electrónico . . . . . . . . . . . . . . . . . . 14

1.4 Otras consideraciones . . . . . . . . . . . . . . . 16

1.5 Bibliografía ........................ 17

2 Transporte térmico en NWs semiconductores 19

2.1 Introducción . . . . . . . . . . . . . . . . . . . . . . . . . 19

2.2 Desarrollo de un modelo predictivo para la conductividad térmica en NWs de silicio y $\mathrm{Si}_{-} 1-\mathrm{xGe} \mathrm{e}_{-} \mathrm{x}$. . . . . . . . . . . . . . . . . 34

2.2.1 El formalismo de Callaway-Holland para la conductividad térmica en semiconductores . . . . . . . . . . 35

2.2.2 Mecanismos de scattering fonónico . . . . . . . . . 39

2.2.3 Cálculo de la conductividad térmica . . . . . . . . . . . 44

2.2.3.1 Validación del modelo para semiconductores volúmi$\cos \ldots \ldots \ldots \ldots \ldots \ldots \ldots$ 
2.2.3.2 Aplicación del modelo a NWs de silicio . . . .

2.2.3.3 Modelo predictivo para la conductividad térmica de NWs de silicio lisos . . . . . . . . . . 54

2.2.3.4 Modelo predictivo para la conductividad térmica de NWs de silicio rugosos . . . . . . . . . 60

2.2.3.5 Modelo para la Conductividad térmica de NWs de silicio-germanio . . . . . . . . . . 69

2.3 Bibliografía . . . . . . . . . . . . . . . . 81

3 Estudio teórico de la distribución de temperatura y su efecto en la señal Raman de NWs semiconductores bajo un haz láser

3.1 Introducción . . . . . . . . . . . . . . . . . . . . . . 87

3.2 Modelado de los efectos térmicos debidos a la interacción láser-NW 89

3.2.1 Modelo FEM para el trasporte térmico de un NW bajo un haz láser . . . . . . . . . . . . . . . . . . . 94

3.2.2 Influencia de la potencia, longitud de onda y medio de inmersión en la temperatura alcanzada en un NW de silicio iluminado por un haz láser . . . . . . . . . . . . . 100

3.2.3 Influencia de las dimensiones geométricas en la temperatura inducida por un haz láser en un NW de silicio . . . . 101

3.2.4 Influencia de la conductividad térmica en la temperatura alcanzada por un NW rugoso de silicio iluminado por un haz láser . . . . . . . . . . . . . . . 105

3.2.5 Efecto de la posición que ocupa el NW dentro del spot del haz láser . . . . . . . . . . . . . . . . . . . 113

3.3 Influencia de la temperatura alcanzada por distintos NWs de silicio en la señal Raman . . . . . . . . . . . . . . . . . . . 116

3.4 Conclusión . . . . . . . . . . . . . . . . . . . . 126

3.5 Bibliografía . . . . . . . . . . . . . . . . 129

4 Espectroscopia Raman en NWs de Silicio y Silicio-Germanio 135

4.1 Introducción . . . . . . . . . . . . . . . . . . . . . . 135

4.2 Espectroscopia Raman en NWs de silicio. . . . . . . . . . . . . . 137

4.2.1 Señal Raman procedente de conjuntos de NWs . . . . . . 137 
4.2.1.1 Influencia del medio en el que se encuentran los NWs .................... 140

4.2.1.2 Análisis del espectro Raman procedente de un conjunto de NWs en función de la potencia del haz incidente . . . . . . . . . . . . . . 143

4.2.2 Señal Raman procedente de NWs individuales . . . . . . 146

4.2.2.1 Señal Raman procedente de un NW inmerso en aire ................. . . 147

4.2.2.2 Espectro Raman procedente de un NW con buena disipación térmica en función de la potencia y longitud de onda del haz láser incidente . . . . 151

4.2.3 Anomalías observadas en los espectros Raman obtenidos de NWs de silicio . . . . . . . . . . . . . . 156

4.2.3.1 Pico adyacente a $495 \mathrm{~cm}^{-1} \ldots \ldots . . . . . .157$

4.2.3.2 Intensidad Raman procedente de los NWs comparada con la intensidad esperada. . . . . . . 167

4.3 Espectroscopia Raman en NWs de $S i_{1-x} G e_{x} \ldots \ldots$. . . . . . . . 171

4.3.1 Efecto de la temperatura inducida por el haz láser en NWs de $S i_{1-X} G e_{X} \ldots \ldots \ldots \ldots$. . . . . . . . 175

4.3.2 Caracterización composicional de NWs de $S i_{1-X} G e_{X}$ por espectroscopia Raman . . . . . . . . . . . . 180

4.3.3 Anomalías encontradas al analizar el espectro Raman de los NWs de $S i_{1-X} G e_{X}$. . . . . . . . . . . . . . . 192

4.3.3.1 Segregación del Germanio en la aleación y existencia de una coraza rica en $\mathrm{Ge}$. . . . . . . . 193

4.3.3.2 Pico adyacente localizado a $\sim 495 \mathrm{~cm}^{-1} \ldots 197$

4.4 Bibliográfia . . . . . . . . . . . . . . . . 203

5 Estudio del Campo electromagnético en el interior de un NW semiconductor excitado con un haz láser polarizado 209

5.1 Introducción . . . . . . . . . . . . . . . . . . . . . . . . . . 209

5.2 Modelado de la interacción electromagnética para el conjunto láserNW-substrato . . . . . . . . . . . . . . . . . . 211 
5.3 Influencia de las dimensiones del NW, longitud de onda y efecto del substrato en el valor de $|E|^{2}$ en el interior de los NWs. . . . . 215

5.4 Intensidad Raman en NWs sobre diferentes substratos . . . . . . . 220

5.5 Efectos de polarización en NWs de silicio. . . . . . . . . . . . 224

5.5.1 Efectos de polarización el los espectros Raman adquiridos en NWs de silicio . . . . . . . . . . . . . . . . 229

5.6 Interacción electromagnética láser-NW y efectos de polarización en NWs de silicio-germanio . . . . . . . . . . . . . 234

5.6.1 Efectos de Polarización en NWs de silicio-germanio . . . 237

5.7 Bibliografía ...................... 241

6 Resumen y Conclusiones 245

7 Apéndices 253

7.1 Apéndice 1: Codigo Mathematica para el cálculo de la conductividad térmica en NWs de silicio, germanio y silicio-germanio . . . . 254

7.2 Apendice 2: Publicaciones generadas durante la realización de este trabajo de investigación . . . . . . . . . . . . . . . 260 


\section{Índice de figuras}

1.1 Diagrama de niveles de energía para los procesos de scattering Rayleigh, anti-Stokes y Stokes. . . . . . . . . . . . . . . 10

1.2 Equipo Raman Horiba Labram HR 800 de Yobin Ybon utilizado para la caracterización experimental de los NWs. A la derecha se muestra un esquema simplificado del equipo. . . . . . . . . . . 12

1.3 A) Equipo de microscopia electrónica LEO 1530 VP SEM utilizado para la caracterización experimental de los NWs. B) Imagen SEM de un NW de silicio adquirida a $5 \mathrm{KV}$ y $12500 \mathrm{X}$ aumentos. C) Imagen SEM a 5KV y 75000X aumentos del mismo NW. . .

2.1 Conductividad térmica experimental en función de la temperatura para el Silicio volúmico [14] y NWs de Silicio con distintos diámetros [11] A), la conductividad térmica está en escala logarítmica. Conductividad térmica de los NWs de silicio en una escala expandida $($ B $) \ldots \ldots \ldots \ldots \ldots \ldots \ldots \ldots$

2.2 Conductividad térmica de dos NWs de germanio por debajo del límite de confinamiento cuántico, puede observarse como aparece una dependencia pseudo-lineal con la temperatura como la observada en NWs de silicio de similares dimensiones $[20]$. . . . .

2.3 Conductividades térmicas de dos NWs de Si de iguales diámetros, $115 \mathrm{~nm}$, siendo uno de ellos de superficie rugosa [2] y otro de superficie lisa $[11] \ldots \ldots \ldots \ldots \ldots$ 


\section{ÍNDICE DE FIGURAS}

2.4 Comparación de la Conductividad térmica experimental de un NW liso de silicio y $115 \mathrm{~nm}$ (circulo negro)[11], frente a un NW liso de silicio-germanio con un 0.4 de germanio y $147 \mathrm{~nm}$ de diámetro (triángulos azules). También se compara la conductividad térmica de dos NWs de silicio-germanio de igual composición pero uno de ellos liso y de $205 \mathrm{~nm}$ de (triángulos blancos) y otro rugoso de 250 $\mathrm{nm}$ (rombos rojos) . . . . . . . . . . . . . . . . .

2.5 Conductividades térmicas experimentales para cristales volúmicos de silicio (A) y germanio (B) -puntos rojos- y conductividad térmica calculada con nuestro modelo de Callaway-Holland. Puede observarse el buen ajuste a los datos experimentales tomados de la referencia $[14]$ en todo el rango de temperaturas . . . . . . . . .

2.6 Conductividades térmicas experimentales de cuatro NWs lisos de silicio con diámetros de $115 \mathrm{~nm}$-círculos blancos-, $56 \mathrm{~nm}$-triángulos negros-, $37 \mathrm{~nm}$-círculos negros- y $22 \mathrm{~nm}$-triángulos blancosobtenidos de la referencia 11. Las lineas muestran la conductividad térmica calcula mediante nuestro modelo para estos NWs, exhibiendo un excelente ajuste en todo el rango de temperatura para el existen datos experimentales . . . . . . . . . . . . .

2.7 Comportamiento de la conductividad térmica en función del parámetro $\zeta-\Lambda_{0}^{\prime}=\zeta \Lambda_{0}$ - que modifica el MFP de los fonones debido a la rugosidad de la superficie de los NWs . . . . . . . . . . .

2.8 Conductividades térmicas experimentales de NWs de silicio rugosos obtenidas de las referencias $[2](A)$ ) y $[22](B)$ y $C$ ) (símbolos rojos) y conductividades térmicas calculadas con nuestro modelo para estos NWs con los parámetros de la tabla 2.3 (lineas) . . . . .

2.9 Velocidades del sonido de las bandas transversal $\left.\left(v_{t}, \mathrm{~A}\right)\right)$ y longitudinal $\left.\left(v_{L}, \mathrm{~B}\right)\right)$ en función del diámetro del NW. Temperaturas de corte de las bandas transversal $\left.\left(\theta_{t}, \mathrm{C}\right)\right)$ y longitudinal $\left.\left(\theta_{L}, \mathrm{D}\right)\right)$ en función del diámetro del NW. . . . . . . . . . . . . . .

2.10 Conductividad térmica experimental para diversos NWs de silicio de la referencia [22] y curvas predichas para la conductividad térmica mediante nuestra aproximación predictiva. . . . . . . . . . 


\section{ÍNDICE DE FIGURAS}

2.11 Conductividades térmicas calculadas para NWs de silicio lisos en un amplio rango de diámetros mediante nuestro modelo predictivo

2.12 Conductividades térmicas a 300 y $400 \mathrm{~K}$ calculadas para NWs de silicio lisos en función del diámetro en el rango de validez del modelo mediante nuestro modelo predictivo (símbolos). Las curvas corresponden al ajuste ajuste dinámico a la expresión 2.32 . . . .

2.13 Datos experimentales de las conductividades térmicas de NWs con distintas características superficiales en función de su diámetro. Puede observarse una distribución bastante aleatoria de las conductividades térmicas en función del diámetro de los NWs A). En $\mathrm{B}$ ( puede verse como la nube de puntos mostrada en A) se ordena cuando se representa $\kappa$ en función de $\zeta \times D$. Lo mismo sucede para 100 y $200 \mathrm{~K}$, ver recuadro interior. . . . . . . . . . . . .

2.14 Cociente $\kappa_{\text {liso }} / \kappa_{\text {rugo }}$ frente a $\zeta^{-1}$, podemos observar la fuerte correlación que aparece entre ambos . . . . . . . . . .

2.15 Representación de los valores teóricos de RMS o $\eta$ frente a los valores experimentales de $\eta$ A) y Representación de los valores teóricos de $L_{\text {correlacion }}$ frente a los valores experimentales de $L_{\text {correlacion }}$ B). Los datos experimentales proceden de [22]. Puede observarse en ambos casos una buena correlación lineal de pendiente 1. . .

2.16 Conductividades térmicas experimentales para el $S i_{1-X} G e_{X}$ volúmico en todo el rango de composición a $300 \mathrm{~K}$. Los datos experimentales proceden de la referencia [73] , A) y referencia [74] B) . . . .

2.17 Conductividades térmicas predichas con el modelo de la referencia [31] (símbolos) y Conductividades térmicas predichas con nuestro modelo para cuatro NWs lisos de germanio de diferentes diámetros.

2.18 Conductividad térmica para 4 cuatro NWs lisos de $S i_{1-X} G e_{X}$ con diferentes composiciones y diámetros procedentes de la referencia[75] (símbolos) y conductividades térmicas calculadas con nuestro modelos para NWs de $S i_{1-X} G e_{X}$ con los mismos diámetros (lineas). Puede observarse el buen acuerdo con los datos experimentales en todos los casos (nótese la escala es logarítmica). . . . . . . . . 76 


\section{ÍNDICE DE FIGURAS}

2.19 A) Conductividad térmica en función de la composición de la aleación para un NW liso de $S i_{1-X} G e_{X}$ de $56 \mathrm{~nm}$ de diámetro (linea), se muestran también las conductividades térmicas experimentales procedentes de [11] y [75], observándose un excelente ajuste a la curva predicha por nuestro modelo. B) Conductividad térmica de NWs de $S i_{1-X} G e_{X}$ en función del diámetro para tres composiciones diferentes. Puede observarse como la influencia del diámetro va disminuyendo según se enriquece la aleación en el componente minoritario . . . . . . . . . . . . . . .

2.20 Conductividad térmica calculada en función de la composición para dos NW de $100 \mathrm{~nm}$, uno liso y otro rugoso. Puede observarse como el efecto de la rugosidad se superpone al de la aleación, obteniéndose una conductividad térmica muy baja . . . . . . . .

3.1 A) Representación tridimensional de la distribución de Airy para la intensidad de un haz láser (Fuente: Wikimedia Commons). B) Representación 2D de la distribución gaussiana equivalente para la intensidad de un haz láser. C) Representación de como son iluminados diferentes NWs de $100 \mathrm{~nm}$ dentro de un spot láser de $1 \mu \mathrm{m}$ de diámetro. . . . . . . . . . . . . . . . . .

3.2 A) Esquema de como afecta el valor de $Q_{a}$ a la absorción de energía en una muestra con una dimensión lateral menor a la longitud de onda del haz láser incidente. B) Índice de refracción complejo para el silicio en función de la longitud de onda necesario para el cálculo de $Q_{a}$, a su lado Reflectividad calculada para el silicio en función de la longitud de onda y la temperatura. C) Cálculo de $Q_{a}$ para NWs de silicio en función del diámetro y para dos longitudes de onda típicas en espectroscopia Raman. Puede observarse como para algunos diámetros se producen resonancias en las que $Q_{a}$ es mayor que $1 . \ldots \ldots \ldots \ldots \ldots \ldots$

3.3 Conductividades térmicas simuladas con el modelo construido en el capítulo 2 para dos NWs de silicio de 115 y $37 \mathrm{~nm}$, tanto con superficies lisas como rugosas. . . . . . . . . . . . 


\section{ÍNDICE DE FIGURAS}

3.4 A) Geometría generada para un NW anclado por uno de sus extremos a un substrato. B) Geometría generada para un NW suspendido entre dos contactos térmicos perfectos. C) Geometría generada para dos NWs que se cruzan en el espacio. D) Aproximación tomada para las superficies de intercambio térmico con el medio que rodea

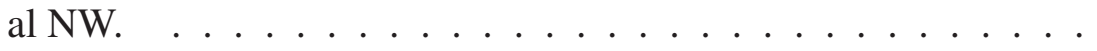

3.5 A) Temperatura alcanzada por un NW liso de silicio de $5 \mu \mathrm{m}$ de longitud y $37 \mathrm{~nm}$ de diámetro completamente inmerso en aire en función de la potencia del haz láser incidente. La linea roja representa la temperatura alcanzada cuando el haz láser tiene una longitud de onda de $514.5 \mathrm{~nm}$ (láser verde), mientras que la curva negra muestra la temperatura en el NW cuando el haz tiene una longitud de onda de $632.8 \mathrm{~nm}$ (láser rojo). A) figura de idéntica descripción a la anterior, pero en este caso el NW está inmerso en agua en vez de en aire. . . . . . . . . . . . . . . . . . 102

3.6 Representación tridimensional de la temperatura alcanzada por un NW de silicio de $5 \mu \mathrm{m}$ de longitud, completamente rodeado por aire, en función del diámetro del NW y de la potencia del láser incidente, el cual tiene una longitud de onda de $632.8 \mathrm{~nm}$. . . . . 104

3.7 Representación tridimensional de la temperatura alcanzada por un NW de silicio liso en función del diámetro y longitud del NW cuando este es iluminado por una haz láser de $200 \mu \mathrm{W}$ y $514.5 \mathrm{~nm}$ de longitud de onda. . . . . . . . . . . . . . . . . 106

3.8 Representación tridimensional de la temperatura alcanzada por un NW de silicio rugoso en función de la conductividad térmica y de su longitud cuando el NW está anclado a un sumidero térmico (substrato) por uno de sus extremos (ver figura 3.4 A) ). El modelo se ha resulto para una configuración dada por un NW de $37 \mathrm{~nm}$ de diámetro, iluminado por un haz láser de $200 \mu \mathrm{W}$, con una longitud de onda de $514.5 \mathrm{~nm}$ y centrado en el punto medio del NW . . . . 108 


\section{ÍNDICE DE FIGURAS}

3.9 A) Distribución de temperatura a lo largo de un NW de silicio con un diámetro de $37 \mathrm{~nm}$ y $5 \mu \mathrm{m}$ de longitud para distintas conductividades térmicas calculadas mediante la ecuación 2.17. La potencia del láser es de $200 \mu \mathrm{W}$ con una longitud de onda de $514.5 \mathrm{~nm}$ y está centrado en el punto medio del NW. B) Distribución de temperatura en un NW idéntico al descrito en A) pero con sus dos extremos conectados a un substrato infinito de silicio. En esté caso la potencia es $500 \mu \mathrm{W}$ en vez de los $200 \mu \mathrm{W}$ utilizados en A) . . .

3.10 Temperatura alcanzada en el punto del NW en el que se focaliza el haz láser representada en función de la posición del punto de focalización del haz láser a lo largo del NW. El NW es de silicio con un diámetro de $37 \mathrm{~nm}$ y $5 \mu \mathrm{m}$ de longitud, estando $4.5 \mu \mathrm{m}$ suspendidos y $0.25 \mu \mathrm{m}$ por cada lado en contacto con el substrato en condición de contacto térmico perfecto. La potencia del haz incidente es de $200 \mu \mathrm{W}$ y su longitud de onda de $514.5 \mathrm{~nm}$. Las lineas son los ajustes obtenidos con la ecuación dada por Tsu et al. en [33] para cada conductividad. . . . . . . . . . . . .

3.11 A) Temperatura inducida en un NW de $37 \mathrm{~nm}$ de diámetro y $5 \mu \mathrm{m}$ de longitud en función de la posición que el NW ocupa dentro del spot del haz láser (ver recuadro interno para una mejor comprensión de la simulación). B) Temperatura inducida en un NW de 37 nm de diámetro y $5 \mu \mathrm{m}$ de longitud en función de la posición a lo largo del NW en la que está enfocado el haz láser. El láser tiene 1 $\mu \mathrm{m}$ de diámetro efectivo, una longitud de onda de $514.5 \mathrm{~nm}$ y 200 $\mu \mathrm{W}$ de potencia en ambos casos. . . . . . . . . . . . 114

3.12 Temperaturas calculadas para un conjunto de NWs de silicio lisos idénticos (37 $\mathrm{nm}$ de diámetro, $5 \mu \mathrm{m}$ de longitud) iluminados simultáneamente por un haz láser de $514.5 \mathrm{~nm}$ de longitud de onda y $200 \mu \mathrm{W}$ de potencia. Se muestran dos configuraciones espaciales de estos NWs, A) y B) separadas por un salto de $100 \mathrm{~nm}$ entre puntos de focalización de haz láser. Puede apreciarse las diferentes temperaturas que se inducen en ambas situaciones pese a la utilización de la misma muestra y la misma fuente de iluminación . . . . 116 


\section{ÍNDICE DE FIGURAS}

3.13 Temperaturas alcanzadas por dos NWs idénticos de $37 \mathrm{~nm}$ de diámetro y $5 \mu \mathrm{m}$ de longitud que se cruzan a 90 grados, estando unos de ellos (U) justo por encima del otro (L) y sin contacto térmico entre ambos (ver esquema de la figura 3.2 C) ). Se muestran 3 configuraciones diferenciadas únicamente por la posición en la que se está focalizando el haz láser en el eje z, estando A) a focalizado a $400 \mathrm{~nm}$ del punto de corte entre los NWs, B) a $140 \mathrm{~nm}$ y C) a 80 $\mathrm{nm}$ (ver esquemas en las figuras como guía aproximada para el ojo). 117

3.14 Espectro Raman calculado para un conjunto de NWs de silicio liso de $5 \mu \mathrm{m}$ de longitud y unos diámetros de $11,6,7$ y $12 \mathrm{~nm}$ respectivamente en dos configuraciones espaciales diferentes ( A) -0.35, $-0.01,0.01$, у $0.35 \mu \mathrm{m}$; B) $-0.05,-0.01,0.015$ y $0.05 \mu m$ del centro del área de iluminación del haz láser respectivamente, el diámetro efectivo del haz láser es $1 \mu \mathrm{m}$ ). En cada figura se muestra la configuración espacial dentro del área de iluminación. En cada caso la curva punteada corresponde al espectro calculado para un único NW de $8 \mathrm{~nm}$ de diámetro para la configuración A)y $10 \mathrm{~nm}$ para la configuración B), mostrando como la redistribución espacial de NWs puede ser confundida con la señal procedente de un único NW de un diámetro determinado. . . . . . . . . . . . . . . . . . 122

3.15 Espectros Raman simulados para un conjunto de NWs iluminados por un haz láser de $632.8 \mathrm{~nm}$ de longitud de onda y una potencia en la muestra de $1.8 \mathrm{~mW}$. Los NWs son de silicio, lisos y con unos diámetros de 12, 10, 11 y $20 \mathrm{~nm}$ respectivamente. En la figura A) están situados a -0.30, 0, 0.15, y $0.4 \mu m$ del centro del spot láser; B) Los NWs están situados a $-0.25,-0.17,0$ y $0.28 \mu \mathrm{m}$ del centro del spot láser. las curvas punteadas representan los ajustes a un único NW que no se calienta de $11 \mathrm{~nm}$ para la configuración A) y de 10 nm para la configuración B) . . . . . . . . . . . . . . 125 


\section{ÍNDICE DE FIGURAS}

3.16 Espectro Raman simulado para un conjunto de NWs de $25 \mathrm{~nm}$ de diámetro y $5 \mu \mathrm{m}$ de longitud situados a una distancia del centro del área de iluminación de $-0.40,-0.20,0.10$ y $0.30 \mu \mathrm{m}$ para A) y -0.45 , $-0.01,0.30$ y $0.40 \mu \mathrm{m}$ para B). Los espectros resultantes muestan diferencias notables dependiendo de como están distribuidos los NWs en el área de iluminación, pese a que la muestra iluminada es la misma. Observesé como incluso aparece una separación del espectro Raman en dos picos únicamente por el efecto de las diferentes temperaturas alcanzadas por cada NW. . . . . . . . . . . 127

4.1 Imagen SEM de una muestra de NWs de silicio tomada sobre el substrato en el que han sido crecidos. En la ampliación se muestra la escala del área de iluminación efectivo del haz láser, puede verse como incluso en una muestra tan densamente poblada como esta, solo unos pocos NWs son iluminados simultáneamente . . . . . . 138

4.2 Espectros Raman obtenidos de una muestra donde son excitados múltiples NWs simultáneamente y el haz láser es enfocado en distintos puntos de la muestra siguiendo una linea con un paso de 0.5 $\mu \mathrm{m}$. El haz láser utilizado es el He-Ne de $632.8 \mathrm{~nm}$ de longitud de onda y con una potencia de $1300 \mu \mathrm{W}$. Los puntos que representan donde está centrado el haz láser deben ser tomados como una guía para el ojo; conviene recordar que el punto donde se sitúa el haz láser se fija por microscopia óptica, y el alineamiento entre ambas técnicas no es perfecto. . . . . . . . . . . . . . 139

4.3 Espectros Raman tomados en la muestra de la figura 4.1 cuando está inmersa en aire (1) y en agua (2). Se muestra también el espectro Raman característico del silicio volúmico para comparar. La potencia utilizada es de $1300 \mu \mathrm{W}$ con el láser de He-Ne de 632.8 nm de longitud de onda . . . . . . . . . . . . . . . . . . 142 
4.4 A) Imagen SEM de dos NWs de silicio paralelos, la linea de puntos corresponde a una guía para ojo que representa el movimiento de láser. Los números representan la posición del láser para los espectros mostrados posteriormente. B) Espectros Raman obtenidos al desplazar el láser a lo largo de la linea transversal mostrada en A) utilizando el láser de Nd:YAG de $532.8 \mathrm{~nm}$ de longitud de onda y $500 \mu \mathrm{W}$ de potencia. C) Espectros Raman adquiridos en las mismas posiciones que en A) con el láser a una potencia de $5 \mu$ W. D) Intensidad Raman normalizada obtenida en cada punto de la linea mostrada en A) para ambas potencias de láser utilizadas. Nótese como tienen un comportamiento gaussiano dado por la distribución de intensidad del haz láser y como al normalizarse los puntos se superponen perfectamente para las dos potencias utilizadas, mostrando que los espectros han sido adquiridos sobre la misma configuración espacial de la muestra. . . . . . . . . . . . . 145

4.5 Espectros Raman adquiridos en la muestra que aparece en la figura 4.4-A) y en el mismo punto para ambas potencias. Puede observarse como para la potencia más elevada aparece una separación en dos picos del espectro Raman y como los segundos ordenes se deforman ostensiblemente por efecto de la temperatura. Cuando se utiliza la potencia más baja puede observarse como ninguno de estos efectos aparece, obteniéndose un espectro prácticamente idéntico al del silicio volúmico. . . . . . . . . . . . . . . . 146 


\section{ÍNDICE DE FIGURAS}

4.6 A) Arriba a la izquierda y con fondo rosa, se muestra la imagen obtenida mediante microscopia óptica confocal y un objetivo 100X de la zona donde se encuentra el NW. En escala de grises se muestra la misma imagen tratada y ampliada. B) Imagen SEM del mismo NW, puede observarse como los perfiles coinciden, si bien las dimension radial estimada de la imagen SEM es muy diferente de lo observado en A). C) Mapas de intensidad Raman obtenidos para el mismo NW utilizando dos potencias diferentes con el láser de Nd:YAG y $532.8 \mathrm{~nm}$ de longitud de onda. Puede observarse como en ambos casos se dibuja el mismo perfil, apareciendo los máximos de intensidad en la misma zona, identificando el punto donde el NW esta centrado en el spot láser gaussiano. . . . . . . . . . . . 148

4.7 A) Imagen SEM del NW de silicio analizado y zona ampliada sobre la que se ha adquirido el espectro. Puede observarse como el NW esta completamente aislado. B) Serie de espectros tomados al variar el haz láser a los largo de la linea transversal que aparecer en a figura A) atravesando el NW. C) Comparación entre uno de los espectros en la zona de máxima intensidad de señal y el espectro correspondiente a al silicio volúmico. Observese como el pico Raman es simétrico pese al desplazamiento y ensanchamiento del pico frente al espectro correspondiente al silicio volúmico. . . .

4.8 A) Perfil de posición del pico Raman en función de la posición que ocupa el NW mostrado en la figura 4.7-A) en el área iluminada por el haz láser. B) Perfil de temperaturas alcanzadas por el NW en función de la posición que ocupa en el área iluminada. Nótese el efecto de la inercia térmica del NW debido al ineficiente intercambio de energía con el aire que lo rodea en la desviación del perfil gaussiano observada en la parte derecha de la figura. . . . . . . 
4.9 Imagen SEM de un NW de silicio depositado sobre un substrato de aluminio. La línea de puntos representa donde se ha centrado el haz láser durante el barrido transversal. B) Espectros obtenidos en los puntos numerados en A) utilizando el láser de Ne:YAG para 500 $\mu \mathrm{W}$ y $5 \mu \mathrm{W}$. Puede observarse como para baja potencia los espectros son independientes de la posición de NW dentro del área de iluminación, mientras que para la potencia más alta se observa un desplazamiento hacia bajas frecuencias y un ensanchamiento por efecto de la temperatura alcanzada en el NW. C) Serie de espectros tomados en los mismos que en B) pero utilizando un láser de He-Ne con una potencia de $1300 \mu \mathrm{W}$. Pude observarse como los espectros son independientes de la posición relativa del NW dentro del área de iluminación, evidenciando que el NW no se está calentando apreciablemente. . . . . . . . . . . . . . . . . 154

4.10 A) Posición del pico Raman en función de la posición del NW respecto al centro del spot láser para diferentes potencias y longitudes de onda. Puede observarse como al bajar la potencia, la posición del pico Raman se va haciendo independiente de la posición que ocupa el NW en el área de iluminación. B) Perfil de temperaturas obtenido de la figura $\mathrm{A})$. La temperatura inducida cuando se utiliza el láser verde más potente se ajusta perfectamente en la parte izquierda a una distribución gaussiana como la predicha en el capítulo 3, mientras que en la parte derecha se puede apreciar el efecto de la inercia térmica mostrado para otros NWs. Nótese la gran diferencia existente al variar la longitud de onda del láser, no apreciándose incremento de temperatura alguno para el láser rojo incluso cuando su potencia nominal es superior a la del láser verde. 155 


\section{ÍNDICE DE FIGURAS}

4.11 Espectros Raman procedentes del NW mostrado en la figura 4.9A). En A) se muestra el espectro adquirido con el láser de Ne:YAG y una potencia de $500 \mu \mathrm{W}$, mostrando como aparece un pico secundario entorno a $495 \mathrm{~cm}^{-1}$ que no tiene correspondencia en el espectro Raman del silicio volúmico, mostrado en en verde en la misma figura. Por otro lado, las intensidades obtenidas para los espectros del NW y del silicio de referencia están representadas en la misma escala, mostrando la enorme intensidad Raman procedente del NW. B) Espectro Raman en el mismo punto del NW en el que se ha obtenido A) pero utilizando en este caso el láser de $\mathrm{He}-\mathrm{Ne}$ y $1300 \mu \mathrm{W}$ de potencia. Puede observarse como aparece el mismo pico adyacente, pero con una intensidad mucho menor que en A). Se muestra también la comparación de intensidades para la misma potencia entre el NW (escala izquierda) y el silicio referencia (escala derecha), mostrando la debilidad de la señal procedente del NW en este caso. . . . . . . . . . . . . . . . . . . . 158

4.12 A) Espectros Raman en función de la potencia del haz láser. Puede observarse como la relación entre las posiciones del pico Raman del silicio y del pico adyacente se mantiene invariable con independencia de la potencia del haz láser. B) Espectros Raman en un rango espectral reducido para observar mejor como para bajas potencias el espectro Raman obtenido es independiente de la potencia del haz láser incidente.

4.13 A) Espectro Raman de un NW de silicio de $52 \mathrm{~nm}$ y $10 \mu \mathrm{m}$ de longitud depositado en un substrato de aluminio. En verde se muestra el espectro procedente del silicio de referencia en la misma escala de intensidades. La imagen SEM corresponden al NW analizado. B) Espectro Raman de un NW de $125 \mathrm{~nm}$ y $5 \mu \mathrm{m}$ de longitud depositado sobre un substrato de germanio. A la izquierda su correspondiente imagen SEM . . . . . . . . . . . . . . . . . 161 
4.14 Imágenes TEM de NWs de silicio crecidos mediante el método VLS sobre substratos de silicio (111). A) NWs crecidos utilizando oro como semilla, en ellos se observan regiones amorfas y densamente pobladas de defectos, especialmente en la zona central. B) NWs crecidos utilizando $A u_{0,5} G a_{0,5}$ como semilla. En este tipo de NWs se observa una estructura monocristalina y libre de defectos.

4.15 A) Espectro Raman adquirido de un NW de silicio crecido con oro como catalizador y depositado sobre aluminio. B) Espectro Raman adquirido sobre un NW de silicio crecido con oro-galio como cata-

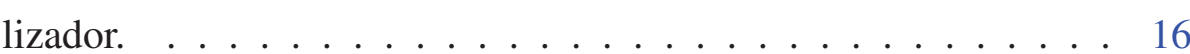

4.16 Espectros Raman adquiridos sobre un NW de silicio crecido con oro-galio y depositado en un substrato de aluminio. Los espectros han sido adquiridos utilizando dos longitudes de onda para el haz láser, $532.8 \mathrm{~nm}(\mathrm{Nd}: Y A G)$ y $632 \mathrm{~nm}(\mathrm{He}-\mathrm{Ne})$ con unas potencias de $500 \mu \mathrm{W}$ y $1300 \mu \mathrm{W}$ respectivamente. La escala de intensidad Raman se muestra en intensidad Raman por unidad de volumen iluminado. La escala perteneciente a los NWs se encuentra a la izquierda, (negro), mientras que los espectros tomados del silicio de referencia se muestran en la escala de la derecha (roja). . . .

4.17 Espectro Raman tomado en un NW de silicio de $110 \mathrm{~nm}$ de diámetro depositado en un substrato de germanio puro. El láser utilizado ha sido el Nd:YAG con una potencia de $500 \mu \mathrm{W}$, encontrándose el NW centrado en el spot láser. Nótese la intensidad Raman del NW en comparación con el pico Raman procedente del substrato de germanio . . . . . . . . . . . . . . . . . . . 172

4.18 Espectro Raman adquirido sobre dos muestras de silicio-germanio con dos composiciones diferentes, $S i_{56} G e_{44}$ y $S i_{45} G e_{55}$. Puede apreciarse la aparición de tres picos diferenciados, correspondientes a los enlaces $\mathrm{Si}-\mathrm{Si}$, $\mathrm{Si}-\mathrm{Ge}$ y $\mathrm{Ge}-\mathrm{Ge}$, y la existencia de otros picos mucho más débiles entre los picos $\mathrm{Si}-\mathrm{Si}$ y Si-Ge. Nótese como al aumentar la cantidad de germanio el pico Si-Si se debilita y se desplaza a bajas frecuencias, mientras que el Ge-Ge aumenta de intensidad y se desplaza a latas frecuencias. . . . . . . . . . . . . 173 


\section{ÍNDICE DE FIGURAS}

4.19 Frecuencias a las que aparecen los picos Raman correspondientes a los enlaces $\mathrm{Si}-\mathrm{Si}(\mathrm{A}), \mathrm{Si}-\mathrm{Ge}(\mathrm{B})$ y Ge-Ge (C) en función de la cantidad de germanio en la aleación (símbolos). Las curvas muestran los ajustes por mínimos cuadrados que permiten interpolar en cada caso la frecuencia en función de la composición. Los datos experimentales proceden de la referencia $[54] \ldots \ldots$. . . . . 174

4.20 Espectros Raman tomados en la muestra compuesta por dos NWs cruzados de $S i_{\sim 85} G e_{\sim 15}$ y mostrada a la derecha (imagen SEM). Puede observarse como dependiendo de la posición que ocupan los NWs en el área iluminada se obtienen unos espectros completamente diferentes. En 1 el láser ilumina de forma exclusiva el NW que se encuentra en contacto con el substrato, mostrando un espectro muy diferente al observado en 2, cuando el láser ilumina a los dos NWs simultáneamente. En este caso se puede apreciar como las diferentes temperaturas alcanzadas por los NWs causan una separación del pico Si-Si en dos. Por último, en 3 el láser ilumina casi exclusivamente al NW que no tiene contacto con el substrato, puede observarse como el pico Si-Si es mucho más grueso que el que aparece en 1 y se encuentra muy desplazado en comparación. El láser utilizado ha sido el Nd:YAG de $532 \mathrm{~nm}$ de longitud de onda y con una potencia de $500 \mu \mathrm{W} \ldots \ldots \ldots \ldots$ 
4.21 A) Espectros Raman adquiridos en el NW de $S i_{\sim 88} G e_{\sim 12}$ mostrado en la imagen SEM. Los espectros se han adquirido a para dos potencias de láser de Nd:YAG, $146 \mu \mathrm{W}$ y $500 \mu \mathrm{W}$ y el NW se encuentra depositado sobre un substrato de aluminio. En 1 se muestra como cuando el NW se se encuentra en el borde del área de iluminación del láser, los picos Si-Si coinciden en posición y forma para ambas potencias. En 2, el NW se encuentra en el centro del área de iluminación, y por tanto la intensidad es mucho mayor que en 1. Puede observarse como para la potencia más alta aparece un desplazamiento muy apreciable hacia bajas frecuencias. B) Posición del pico Si-Si en función de la posición del NW en el área de iluminación. Puede observarse como para la potencia más alta aparece un perfil de temperatura dominado por la distribución gaussiana de la potencia del láser. El perfil para la potencia más baja es independiente de la posición en el área, lo que indica que para esa potencia no se induce una temperatura apreciable en el NW, siendo óptima para la caracterización del NW. Nótese como aparece de nuevo el pico adyacente que se observaba en los espectros adquiridos en NWs de silicio. Esto será tratado en un sección posterior. . . . . . 179

4.22 Imagen TEM de un NW silicio-germanio de la muestra caracterizada por una composición media de $S i_{\sim 88} G e_{\sim 12}$. Puede observarse como la composición no es homogénea a lo largo del NW; la aleación es más débil en germanio cuanto más nos aproximamos a la bola de oro-galio. Debido al proceso de crecimiento la parte más próxima a la bola no contiene nada de germanio. . . . . . . . . . 181 


\section{ÍNDICE DE FIGURAS}

4.23 Imagen SEM del NW de silicio-germanio la muestra con una composición media del $12 \%$ de germanio. Las imágenes numeradas de 1 al 5 muestran los espectros obtenidos en los puntos señalados en la imagen SEM, separados aproximadamente $1 \mu \mathrm{m}$ para asegurar que se iluminan zonas independientes en cada paso. En 1 se muestran los espectros adquiridos a $500 \mu \mathrm{W}$ y a $50 \mu \mathrm{W}$, mostrando que el espectro no cambia al modificar la potencia, por lo que no se induce una temperatura apreciable en el NW con ninguna de las dos potencias. En azul aparece ampliada la ventana espectral correspondiente a los picos Ge-Ge y Si-Ge ya que su intensidad es muy débil para esta composición de la aleación.

4.24 A) Perfiles de posición de los picos Si-Si y Ge-Ge en función del punto a los largo de la longitud del NW en el que se focaliza el haz láser. El pico Si-Si se lee en la escala de la izquierda (roja) y el Ge-Ge en la derecha (negra). Las lineas son únicamente una guía para el ojo. B) Composiciones obtenidas de las frecuencias mostradas en la figura A) para los picos Si-Si y Ge-Ge. Puede observarse la enorme discrepancia entre las composiciones obtenidas de cada pico. Nótese también la buena correspondencia obtenida entre la determinación de la composición mediante el pico Si-Si y la composición determinada para los NWs de esta muestra por EDX. 185

4.25 Imagen SEM de un NW de silicio-germanio de la muestra correspondiente a una composición del $12 \%$ de germanio. Los números muestran la zona iluminada en donde se han tomado los espectros numerados que aparecen a continuación. En azul aparece una representación en el rango espectral correspondiente a los picos GeGe y Si-Ge para su mejor apreciación. . . . . . . . . . . . . . 186 


\section{ÍNDICE DE FIGURAS}

4.26 A) Frecuencia a la que aparecen los picos $\mathrm{Si}$-Si (círculos rojos) y Ge-Ge (cuadrados negros) en función de la posición a la que se centra el spot láser a lo largo del NW. Nótese la falta de correlación evidente entre las posiciones de ambos picos. B) Perfil de composición del NW a lo largo de su longitud calculada a través de las frecuencias de los picos Si-Si (rojo) y Ge-Ge (negro). Puede observarse la gran discrepancia entre las composiciones determinadas a través de cada pico. En ambas figuras el pico Si-Si se lee en la escala izquierda (roja) y el Ge-Ge en la derecha. . . . . . . . . . . 188

4.27 Imagen SEM de un NW de silicio-germanio procedente de la muestra con una composición media del $15 \%$ de germanio. El NW se encuentra depositado en un substrato de aluminio, tiene unas dimensiones de $90 \mathrm{~nm}$ de diámetro en su parte más gruesa y $\sim 80$ $\mathrm{nm}$ en su parte más fina, por otro lado su longitud es de $\sim 4.5 \mu \mathrm{m}$. En los espectros obtenidos en las distintas partes del NW podemos observar la gran intensidad relativa del pico Ge-Ge respecto al pico Si-Si y la inhomogéneidad de dicha intensidad relativa a lo largo del NW. Por otro lado llama la atención que no esté presente el pico suplementario a $\sim 495 \mathrm{~cm}^{-1}$. . . . . . . . . . . . 189

4.28 A) Frecuencias a las que aparecen los picos $\mathrm{Si}-\mathrm{Si}$ (círculos rojos) y Ge-Ge (cuadrados negros) en función del punto donde se centra el haz láser a lo largo del NW. B) Composiciones determinadas a través de las frecuencias mostradas en A). Pude observarse como la composición determinada por el pico GE-Ge muestra que tenemos un NW de germanio puro, mientras que la determinada por el $\mathrm{Si}-\mathrm{Si}$ muestra una composición menor a la determinada por EDX para esta muestra. 


\section{ÍNDICE DE FIGURAS}

4.29 Imagen SEM de un grupo de NWs de silicio-germanio pertenecientes a la muestra caracterizada por un $15 \%$ de germanio. Puede observarse como los NWs están alineados longitudinalmente y suficientemente separados como para poder analizar por separado sus espectros. Nótese como el pico Ge-Ge aparece a una frecuencia muy próxima al germanio puro y con una intensidad muy elevada para la poca cantidad de germanio presente en la aleación. . . . . 191

4.30 A) Frecuencias a las que aparece los picos $\mathrm{Si}-\mathrm{Si}$ y Ge-Ge para cada región iluminada mostrada en 4.19. Composiciones calculadas a través de las frecuencias mostradas en A) . . . . . . . .

4.31 Proporciones de germanio y de silicio en un NW perteneciente a la muestra caracterizada por una composición media del $12 \%$ de germanio. Puede observarse como el germanio no está distribuido de forma homogénea, sino que aparecen zonas ricas en germanio en la zona cercana a la superficie (ver esquema de la estructura core-shell que presentan los NWs). . . . . . . . . . . . . . . . 194

4.32 A) Espectro Raman procedente de la referencia [67] adquirido sobre un NW de silicio-germanio con estructura core-shell donde la corteza (shell) tiene cerca de $40 \mathrm{~nm}$ de grosor y esta compuesta únicamente por germanio, mientras que el núcleo (core) tiene menos de $20 \mathrm{~nm}$ y está compuesto por una aleación de silicio-germanio muy rica en silicio. B) Espectro Raman adquirido en un NW de la muestra $S i_{85} G e_{15}$. Nótese la similitud entre ambos espectros pese a las enormes diferencias estructurales y composicionales que existen entre ambos . . . . . . . . . . . . . . . . . . . 194 


\section{ÍNDICE DE FIGURAS}

4.33 A) Espectros Raman obtenidos en laminas de silicio-germanio con un $12 \%$ y un $60 \%$ de germanio respectivamente. Puede observarse como ninguna de las dos se corresponde con los espectros obtenidos en los NWs con un porcentaje de germanio determinado por el pico Si-Si del $12 \%$ y del $60 \%$ mediante el pico Ge-Ge. B) Comparación del espectro obtenido para el NW de la figura 4.25 y del espectro resultante de dos contribuciones, una con un $12 \%$ de germanio y otra con un $60 \%$ de germanio. Puede observarse la similitud de este espectro con el obtenido del NW pese a la brusquedad de la aproximación, ya que el peso dado a las señales es aproximado.

4.34 Variación de los espectros Raman obtenidos cuando el haz láser barre transversalmente un NW de $S i_{85} G e_{15}$ (números) y cunado el barrido se hace longitudinalmente (letras). Nótese la diferencia en las intensidades relativas del pico $\mathrm{Ge}-\mathrm{Ge}$ frente al $\mathrm{Si}-\mathrm{Si}$ en el corte transversal.

4.35 A) Espectros Raman correspondientes a tres NWs de silicio-germanio pertenecientes a muestras con tres proporciones de germanio en la aleación diferentes. Puede observarse como el pico suplementario aparece en la misma posición en cada caso $\left(495 \mathrm{~cm}^{-1}\right)$. B) Espectros Raman obtenidos en los NWs de silicio y silicio-germanio mostrados en las imágenes SEM. Nótese como las posiciones de las frecuencias de los picos $\mathrm{Si}$-Si se desplazan en función de la composición con respecto a los picos obtenidos los NWs de silicio, mientras que los picos suplementarios se muestran invariantes a la existencia de germanio . . . . . . . . . . . . . . . . . . . 199

4.36 Posición del pico adyacente en un NW de silicio-germanio en función de la posición que ocupa el NW dentro del área de iluminación para dos potencias del láser Nd:YAG diferentes. Puede observarse como aparece el mismo perfil para el pico adyacente que para el pico Si-Si (Izquierda bajo la imagen SEM), por lo que ambos presentan la misma dependencia con la temperatura . . . . . . . . . 200 


\section{ÍNDICE DE FIGURAS}

4.37 A) Intensidad relativa del pico 495 frente al pico Si-Si a lo largo de un NW de $S i_{88} G e_{12}$. B) Intensidad relativa del pico 495 frente al pico Si-Si en función de la posición que ocupa el NW en el área de iluminación habiéndose eliminado los efectos de temperatura en el experimento . . . . . . . . . . . . . 202

5.1 A) Dominio de simulación implementado en $C O M S O L$ para la resolución del problema electromagnético resultante de iluminar NWs semiconductores con un haz láser. El NW se encuentra depositado sobre un volumen que hace las veces de substrato, cuando se simula un NW rodeado completamente por aire este volumen desaparece. B) Ampliación del NW mostrado en A), puede observarse como el modelo permite resolver el problema tanto para NWs de silicio (coraza y núcleo idénticos) como para NWs de germanio con germanio segregado (Corteza de germanio y núcleo de siliciogermanio $\ldots \ldots \ldots \ldots \ldots \ldots \ldots \ldots \ldots$

5.2 A) $|E|^{2}$ normalizado calculado por FDTD en [7] en el interior de NWs de silicio iluminados con un haz láser de 532 nm y situados sobre un substrato de oro. B) Solución para las mismas condiciones obtenida con el modelo FEM aquí desarrollado. Puede observarse la perfecta correspondencia entre ambas soluciones. C) Eficiencia de absorción calculada a través del formalismo de Mie y valor normalizado de $|E|^{2}$ obtenido mediante el modelo FEM. . . . . . . . 216 


\section{ÍNDICE DE FIGURAS}

5.3 A) Distribución de $|E|^{2}$ en función del substrato para dos NWs de silicio de 110 y $40 \mathrm{~nm}$ de diámetro que son iluminados por un haz láser de $532 \mathrm{~nm}$. B) valor máximo de $|E|^{2}$ en el interior de los NWs de silicio en función de su diámetro y del substrato sobre el que se apoyan. Se ha considerado $E_{0}=1$ en la ecuación 5.2 por simplicidad y sin perdida de generalidad del resultado. C) Aumento del valor de $|E|^{2}$ integrado en la sección del NW de los NWs sobre un substrato respecto al valor de $|E|^{2}$ procedente del NW rodeado por aire. Nótese como pueden aparecer incrementos en $|E|^{2}$ de hasta tres ordenes de magnitud para algunos diámetros y substratos, lo que implica un aumento en la señal Raman de tres ordenes de magnitud. 218

5.4 Misma figura que la mostrada en 5.3-A, B y C pero cambiando la longitud de onda de la radiación incidente a $632.8 \mathrm{~nm}$. Obsérvese que las resonancias en $|E|^{2}$ siguen apareciendo, pero se desplazan en todos los casos a diámetros mayores. . . . . . . . . . .

5.5 Distribución de $|E|^{2}$ y espectros Raman para dos NWs sobre aluminio y germanio. Puede observarse como para el NW de $52 \mathrm{~nm}$ depositado sobre aluminio el valor de $|E|^{2}$ es más de cuatro veces más intenso que para el NW de $125 \mathrm{~nm}$ depositado sobre germanio, lo que corresponde a un valor integrado de $|E|^{2} 1.6$ mayor en

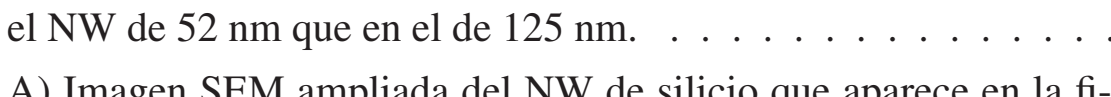

5.6 A) Imagen SEM ampliada del NW de silicio que aparece en la figura 4.16. Debajo Imagen procedente del microscopio óptico. B) Espectros Raman para el NW de $52 \mathrm{~nm}$ depositado sobre aluminio y para el silicio de referencia, ambos iluminados con láser He-Ne $(\lambda=632 \mathrm{~nm})$ (el silicio de referencia se lee en la escala derecha). Al lado la distribución de $|E|^{2}$ en el NW. C) Espectros Raman para el NW de $52 \mathrm{~nm}$ depositado sobre aluminio y para el silicio de referencia, ambos iluminados con láser Nd:YAG $(\lambda=532 \mathrm{~nm})$. Al lado la distribución de $|E|^{2}$ en el NW. Nótese que sólo son directamente comparables las intensidades de las medidas realizadas en muestras bajo las mismas condiciones de excitación, no siendo directamente comparables medidas realizadas con longitudes de onda diferentes. 


\section{ÍNDICE DE FIGURAS}

5.7 A) Imagen SEM de un conjunto de 3 NWs de silicio depositados sobre aluminio, el NW principal aparece próximo a $90^{\circ} . \mathrm{B}$ )Diámetro del NW principal medido a partir de la imagen SEM. C)Imagen obtenida con el microscopio confocal y el objetivo $100 \mathrm{X}$ con la muestra dispuesta de tal modo que el NW principal está alineado con el eje de polarización de la luz incidente. D)- Imagen obtenida al rotar la muestra $90^{\circ} \ldots \ldots \ldots \ldots . \ldots . \ldots 226$

5.8 Valor de $|E|^{2}$ integrado en función del ángulo que forman NWs de distintos diámetros con el eje de polarización del láser incidente. El estudio se ha realizado para dos longitudes de onda diferentes, 532 nm y 632 nm. Nótese que todos los gráficos son Log-Log salvo el perteneciente al NW de $125 \mathrm{~nm}$ iluminado por el haz de $532 \mathrm{~nm}$.

$5.9|E|^{2}$ integrado en un NW de silicio de $187 \mathrm{~nm}$ de diámetro. Pueden observarse las diferentes distribuciones de $|E|^{2}$ en el interior del nanohilo en función del substrato en el que está depositado el NW. El gráfico polar muestra el valor de $|E|^{2}$ en función de $\alpha$ para cada substrato. Nótese que enorme efecto que introduce el substrato en la dependencia de $|E|^{2}$ con $\alpha$ llegándose a invertir el comportamiento pseudo-dipolar cuando el substrato es aluminio. . . . . . .

5.10 A)- Espectros Raman obtenidos en el NW principal de la figura 5.6 en función del ángulo $\alpha$ cuando se ilumina el NW con el láser He-Ne de 632.8 nm de longitud de onda. B) Patrón de intensidades Raman normalizadas obtenidas al girar la muestra para valores de $\alpha$ entre $0^{\circ}$ y $180^{\circ}$. Nótese como aparece el comportamiento predicho por el modelo teórico del acoplamiento electromagnético . . . . . 232 
5.11 Espectros Raman obtenidos en el NW principal de la figura 5.6 en función del $\alpha$ cuando se ilumina el NW con el láser Nd:YAG de 532.8 nm de longitud de onda. A) Patrón de intensidad Raman del pico principal del silicio en función de $\alpha \in\left[0^{\circ}, 180^{\circ}\right] \cup\left[270^{\circ}, 360^{\circ}\right]$. B) Patrón de intensidad Raman del pico principal del silicio en función de $\alpha \in\left[0^{\circ}, 180^{\circ}\right] \cup\left[270^{\circ}, 360^{\circ}\right]$ y del pico 495 en $\alpha \in$ $\left[180^{\circ}, 270^{\circ}\right]$. Nótese como el patrón de intensidades experimentales difiere del patrón obtenido para $|E|^{2}$ en las mismas condiciones experimentales. C) Representación en escala Log-Log del patrón mostrado en B) y el valor calculado para $|E|^{2}$ normalizado. D) Representación en escala Log-Log del patrón mostrado en B) y mejor ajuste utilizando el patrón de teórico de $|E|^{2}$. F) Representación en escala Log-Log del patrón mostrado en B) y mejor ajuste a $|E|^{4}$ como la propuesta en $[4]$ y $[7] \ldots \ldots$. . . . . . . . .

5.12 Distribución de $|E|^{2}$ en el interior de un NW de $85 \mathrm{~nm}$ de diámetro como el mostrado en la imagen SEM que acompaña a la figura. Las figuras numeradas corresponden a la posición que ocupa el NW en el haz láser en pasos de $500 \mathrm{~nm}$, que son los puntos donde se han adquirido los espectros Raman numerados de idéntica forma (ver esquema de la posición que ocupa el NW en la zona iluminada en la imagen SEM). Nótese como la distribución de $|E|^{2}$ se concentra en distintas partes del NW en función de la posición que ocupa el hilo en el área iluminada por el haz láser . . . . . . . . . . . . 236

5.13 Serie de espectros Raman adquiridos para el NW de silicio-germanio de la figura 5.12 para dos potencias distintas del láser Nd:YaG en función del ángulo formado por el eje del NW y el eje de polarización. En el mapa polar puede observarse la dependencia de la intensidad del pico Si-Si con dicho ángulo, mostrándose también el patrón de $|E|^{2}$ simulado para este NW en las mismas condiciones experimentales. Nótese como la intensidad Raman obedece el patrón marcado por $|E|^{2}$, obteniéndose un mejor ajuste cuando se considera que el NW tiene una pequeña coraza de silicio segregado (4 nm). 



\section{Índice de cuadros}

2.1 Parámetros utilizados en el cálculo de la conductividad térmica del silicio volúmico y germanio volúmico . . . . . . . . . . . . 47

2.2 Parámetros utilizados en el cálculo de la conductividad térmica de los NWs lisos de silicio medidos en la referencia 11 . . . . . . . 49

2.3 Parámetros utilizados en el cálculo de la conductividad térmica de los NWs rugosos de silicio medidos en las referencias 2 y $22 \ldots 54$

2.4 Parámetros obtenidos de los ajustes de las curvas mostradas en la figura $2.9 \ldots \ldots \ldots \ldots$

4.1 Condiciones de crecimiento para cada una de los NWs de siliciogermanio analizados . . . . . . . . . . . . . . . . 180

5.1 Valores de los indices de refracción utilizados en las simulaciones para cada material procedentes de la referencia $[18 \ldots \ldots$ 



\section{Estructura de la tesis}

En esta tesis se analizan diversos aspectos de la caracterización por espectroscopia Raman de nanohilos (NWs) semiconductores de silicio y silicio-germanio. La estructura de la tesis responde de forma jerárquica al grado de interconexión entre los resultados de cada capítulo; es decir, la comprensión del capítulo 5 requiere de los resultados alcanzados en el capítulo 4, que a su vez no pueden explicarse sin el estudio teórico realizado en el capítulo 3, para el cual es fundamental la caracterización de la conductividad térmica llevada a cabo en el capítulo 2. Para que esta estructura piramidal sea más fácil de seguir cada capítulo incorpora su propia introducción recopilando tanto el estado del arte del tema tratado en dicho capítulo, así como de la descripción teórica necesaria para comprender los resultados y conclusiones alcanzados. La adopción de esta estructura implica que el capítulo 1, o introducción, únicamente describe algunos conceptos comunes a la mayoría de los capítulos restantes; i.e. en la introducción se describe la teoría sobre la que descansa la espectroscopia Raman y se realiza una breve descripción del equipo experimental utilizado para la realización de esta tesis.

El capitulo 2 muestra el modelo construido para predecir la conductividad térmica en NWs de silicio y silicio-germanio. Este modelo incorpora el efecto introducido por el diámetro de los NWs, las características morfológicas de la superficie y el efecto de la composición en la aleación. De este modo, podemos predecir la conductividad térmica de los NWs de silicio y silicio-germanio en un amplio rango de temperaturas. Este resultado no sólo es fundamental para realizar el estudio teórico de la interacción térmica láser-NW del capítulo 3, sino que tiene relevancia por sí mismo, ya que el estudio de la conductividad térmica de los NWs semiconductores es uno de los campos más explotados actualmente en la literatura científica 
sobre NWs semiconductores.

Una vez conocida la conductividad térmica de los NWs, es posible llevar a cabo el modelado de la interacción térmica láser-NW que sucede en espectroscopia micro-Raman, tal y como se muestra en el capítulo 3. Este modelado se realiza mediante un análisis por elementos finitos (FEM) construido sobre el software de COMSOL Multiphysics. Con este modelo se han estudiado los posibles factores que intervienen en la interacción térmica láser-NW, teniéndose en cuenta para ello que el NW tiene unas dimensiones menores a la longitud de onda incidente y también mucho menores al área de iluminación del haz láser.

En el capítulo 4, se muestran los resultados experimentales de diferentes análisis Raman efectuados sobre NWs de silicio y silicio-germanio, mostrándose experimentalmente lo calculado teóricamente en el capítulo 3; e.g. la enorme influencia en el espectro Raman de la temperatura inducida en los NWs, el efecto del medio en el que se encuentran los NWs, las diferencias entre iluminar NWs únicos o conjuntos de NWs, etc... Además, se establece un criterio que permite asegurar la eliminación del efecto de la temperatura en los NWs durante la caracterización Raman. Gracias a esto, se puede abordar en la segunda parte del capítulo 4 la caracterización composicional en NWs de silicio-germanio mediante espectroscopia micro-Raman.

En la caracterización experimental de los NWs de silicio y silicio-germanio realizada en el capítulo 4 aparecen algunos resultados anómalos; e.g. picos suplementarios, enormes intensidades Raman, espectros compuestos... Algunos de estos efectos han sido reportados por otros autores, pero no explican satisfactoriamente lo observado en los NWs analizados en este trabajo. Para entender estos efectos anómalos, en el capítulo 5 se realiza un análisis por elementos finitos de la interacción electromagnética láser-substrato-NW, implementada en el software de COMSOL Multiphysics. Gracias a esto, se demostrará que estos efectos anómalos proceden en su mayoría de esta interacción. Además, en este capítulo se muestra como el efecto de que el diámetro del NW sea menor a la longitud de onda incidente introduce una dependencia en la intensidad de la señal Raman con la alineación entre el eje de polarización del NW y del eje de polarización del haz láser. Este efecto se explora tanto experimentalmente como teóricamente en el capítulo 5. 
Et dixit: nunc coepi...

anonymous

CAPÍTULO

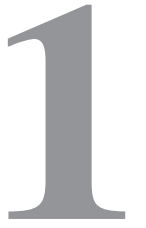

\section{Introducción}

Los nanohilos semiconductores (NWs) son estructuras cuasi-unidimensionales con un gran potencial para convertirse en los cimientos sobre los que construir los nanodispositivos electrónicos, optoelectrónicos, y termoeléctricos del futuro $[1,2]$. Una característica común a todos los NWs semiconductores es que muchas de sus propiedades físicas no tienen el mismo comportamiento que se observa en sus equivalentes volúmicos $[3-5]$. Esto sucede fundamentalmente por que los diámetros característicos son menores a pocas centenas de nanómetros, y por tanto están por debajo de las longitudes características de muchos fenómenos físicos; e.g. longitud de onda de los fotones, camino libre medio de los fonones, longitudes de difusión de los excitones, tamaño de los dominios magnéticos etc.... Además, los defectos presentes en la estructura, tales como dislocaciones, impurezas, rugosidad de la superficie, etc..., son mucho más relevantes en estas estructuras que en materiales volúmicos [6]. Esta influencia del tamaño de los NWs en sus características físicas, implica que antes de poder desarrollar dispositivos basados en NWs, primero es necesario caracterizarlos en profundidad para conocer su respuesta ante diferentes estímulos; e.g. el paso de una corriente eléctrica a través de ellos, su respuesta cuando se los somete a campos electromagnéticos o la gestión de gradientes térmicos en su interior. 


\section{INTRODUCCIÓN}

Dentro de las herramientas de caracterización existentes y aplicables a estas estructuras, la espectroscopia Raman, dado su carácter no destructivo y su resolución micrométrica, resulta muy adecuada para estudiar la estructura cristalina de los NWs, su pureza, orientación cristalográfica, composición, concentración de dopantes, comportamiento fonónico, temperatura, etc...[7]. Esta técnica ha sido ampliamente aplicada en la caracterización de NWs semiconductores [8 - 11]; sin embargo, dado que los NWs presentan unas características geométricas muy especiales; e.g. tamaño menor a longitud de onda y área de iluminación del láser incidente, la forma de analizar correctamente la información obtenida a través de esta caracterización requiere ser estudiada en profundidad.

\subsection{Nociones básicas sobre el scattering Raman}

En 1923, A. Smekal mostró teóricamente como la radiación electromagnética que atraviesa un medio no sufre únicamente fenómenos de scattering elásticos, o Rayleigh, sino que también deben aparecer eventos de scattering inelásticos causados por los movimientos traslacionales o rotacionales de los átomos o moléculas que conforman el medio atravesado por la radiación electromagnética [12]. Posteriormente, C. V. Raman demostró experimentalmente la existencia de este fenómeno, ganando por ello el premio novel de física en 1930 [13].

La probabilidad de que ocurran estos eventos de scattering inelástico, o Raman, es muy baja comparada con los eventos Rayleigth [14]; dada la baja intensidad de esta emisión, en los primeros tiempos de esta técnica el análisis de la radiación Raman era una tarea muy compleja y delicada que requería de enormes tiempos de adquisición, resultando poco atractiva como técnica de caracterización.

Posteriormente, y gracias al desarrollo de los haces láser como fuente de excitación y de los dispositivos de carga acoplada (CCD) para la detección de la radiación, la adquisición de la radiación Raman se ha optimizado y simplificado enormemente, convirtiendo a esta técnica de caracterización en una herramienta fundamental para estudiar las propiedades de la materia, y actualmente su campo de aplicación se extiende a la biología, geología, arqueología, química, física del estado sólido, etc... La espectroscopia Raman aplicada a NWs de silicio y silicio-germanio es la com- 
ponente principal y el hilo conductor de esta tesis, y por tanto es necesario describir su naturaleza para una mejor comprensión de los capítulos siguientes.

\subsubsection{Scattering Raman desde un punto de vista clásico}

La descripción del scattering Raman puede realizarse desde el punto de vista del electromagnetismo clásico [15 - 17]. A modo de simplificación, y sin perdida de generalidad, asumiremos que el haz que ilumina la muestra puede describirse como un campo electromagnético que varía en el tiempo con una frecuencia $\omega$ :

$$
\vec{E}=\overrightarrow{E_{0}} \cos (\omega t)
$$

Siendo $\overrightarrow{E_{0}}$ la amplitud del campo eléctrico que caracteriza la radiación incidente. Cuando el haz incidente ilumina un material dieléctrico como el silicio o el siliciogermanio, en su interior se induce un momento dipolar debido al campo eléctrico aplicado:

$$
\vec{P}=\vec{\chi} \cdot \vec{E}
$$

Donde $\vec{\chi}$ es la susceptibilidad del material, que en general va a ser un tensor de orden dos. La susceptibilidad $\vec{\chi}$ está modulada por las fluctuaciones de los átomos sobre sus posiciones de equilibrio en la red. Estas fluctuaciones están caracterizadas a su vez por la vibraciones de la red, y por tanto relacionadas con los fonones de la red. Estos fonones pueden describirse en sus coordenadas normales, descritas a través de la función de Bloch como [18]:

$$
Q_{j}=A_{j} e^{ \pm\left(i k_{j} r-Q_{j} t\right)}
$$

De tal forma que la modulación de la susceptibilidad del material puede expandirse en serie de Taylor respecto a las coordenadas normales de los fonones alrededor de su posición de equilibrio $\vec{\chi}_{0}$ como:

$$
\vec{\chi}=\vec{\chi}_{0}+\sum_{j}\left(\frac{\partial \vec{\chi}}{\partial Q_{j}}\right)_{0} Q_{j}+\frac{1}{2} \sum_{j, i}\left(\frac{\partial \vec{\chi}}{\partial Q_{j} \partial Q_{i}}\right)_{0} Q_{j} Q_{i}+\ldots
$$




\section{INTRODUCCIÓN}

Para pequeñas fluctuaciones alrededor de la posición de equilibrio, y por tanto en aproximación eléctrica armónica, sólo es necesario considerar un modo normal de vibración $j$, y por tanto:

$$
\vec{\chi}=\vec{\chi}_{0}+\left(\frac{\partial \vec{\chi}}{\partial Q_{j}}\right)_{0} Q_{j}
$$

Bajo la aproximación eléctrica armónica puede considerarse que la coordenada normal $Q_{j}$, cuya forma está dada por la ecuación 1.3, puede simplificarse como:

$$
Q_{j}=Q_{j 0} \cos \left(\omega_{j} t\right)
$$

Esto permite aproximar la polarización inducida en la muestra iluminada a través de la expresión:

$$
\vec{P}=\vec{\chi}_{0} \cdot \vec{E}_{0} \cos \left(\omega_{j} t\right)+\left(\frac{\partial \vec{\chi}}{\partial Q_{j}}\right)_{0} Q_{j 0} \vec{E}_{0} \cos \left(\omega_{j} t\right) \cos (\omega t)
$$

Esta ecuación puede reformularse de tal modo que aparezcan claramente identificados los diferentes términos del scattering, tanto Rayleigth como Raman, mediante una relación trigonométrica para los cosenos [16]:

$$
\vec{P}=\vec{\chi}_{0} \cdot \vec{E}_{0} \cos \left(\omega_{j} t\right)+\left(\frac{\partial \vec{\chi}}{\partial Q_{j}}\right)_{0} Q_{j 0} \vec{E}_{0} \frac{1}{2}\left\{\cos \left(\left(\omega-\omega_{j}\right) t\right)+\cos \left(\left(\omega-\omega_{j} t\right)\right\}\right.
$$

En esta ecuación puede observarse claramente la presencia de tres frecuencias diferentes, y asumiendo que la muestra iluminada irradie energía como un dipolo, la luz emitida por la muestra está caracterizada por estas mismas tres frecuencias principalmente. El primer termino, caracterizado por una frecuencia $\omega$ es el correspondiente a los eventos elásticos o Rayleigth, y en él la frecuencia de la luz emitida coincide con la frecuencia de la luz incidente. El segundo termino en la ecuación 1.8 engloba los eventos inelásticos, o Raman, y está caracterizado por las dos frecuencias, $\omega-\omega_{j} \mathrm{y} \omega+\omega_{j}$, correspondientes a los eventos Raman Stokes y anti-Stokes respectivamente. Las diferencias de frecuencias $\omega \pm \omega_{j}$ se denominan desplazamiento Raman (Raman shift) y típicamente se expresan en unidades de $\mathrm{cm}^{-1}$.

Para obtener las intensidades Stokes y anti-Stokes emitidas por una muestra ilumi- 
nada por un campo electromagnético desde un punto de vista clásico, uno puede recurrir al formalismo desarrollado por M. Cardona et al. en [14], según el cual,la energía irradiada por unidad de tiempo por la muestra puede expresarse como:

$$
\frac{d \mathbf{I}}{d \Omega}=\frac{\omega^{4}}{(4 \pi)^{2} \epsilon_{0} c^{3}}\left|\vec{e}_{s} \cdot \vec{P}\right|^{2}
$$

Donde I es la intensidad irradiada por la muestra, $\Omega$ el elemento de ángulo sólido, y $\omega$ la frecuencia del momento dipolar $\vec{P}$ inducido en el material.

En el tratamiento clásico, en el cual el medio tiene unas dimensiones mayores a la longitud de onda de la radiación incidente, este momento dipolar inducido en el interior del material está determinado por el campo eléctrico incidente a través de $\vec{P}=\epsilon_{0} \vec{\chi} \vec{e}_{i} E_{i}$, siendo por tanto la intensidad irradiada por el material $\mathbf{I}=\frac{1}{2} \epsilon_{0} c E_{i}^{2}$. Sin embargo, esta aproximación para la intensidad irradiada por el material iluminado no es válida para estructuras como los NWs [7]. En esta aproximación se asume que el campo eléctrico dentro del material, y que por tanto origina el momento dipolar en el material, es igual al de la radiación incidente; sin embargo y como veremos en el capítulo 5, debido a las diferencias entre las constantes dieléctricas del medio, del substrato y del NW, y dadas las dimensiones del NW frente a la longitud de onda del campo electromagnético incidente, en el interior del NW el campo eléctrico inducido puede ser muy diferente del campo eléctrico de la radiación incidente.

Pese a esto, y dado que la sección eficaz para los eventos de scattering Raman es independiente del campo eléctrico en el interior de la muestra, este tratamiento sigue siendo válido, pudiéndose expresar la sección eficaz diferencial como:

$$
\frac{d \sigma}{d \Omega}=\frac{\omega^{4} V^{2}}{(4 \pi)^{2} c^{4}}\left|\vec{e}_{s} \cdot \overrightarrow{\hat{\chi}} \cdot \vec{e}_{i}\right|^{2}
$$

Donde $\hat{\chi}$ es la susceptibilidad independiente del volumen de material iluminado por el haz incidente o volumen de scattering, $V$. Si en esta ecuación se introduce la aproximación para $\vec{\chi}$ dada en la ecuación 1.18 y se tienen en cuenta las distribuciones de población para los fonones, se pueden extraer las secciones eficaces para 


\section{INTRODUCCIÓN}

los eventos Raman Stokes y anti-Stokes, como [14]:

$$
\begin{gathered}
\left(\frac{d \sigma}{d \Omega}\right)_{\text {Stokes }}=\left(\left(\frac{1}{e^{\frac{\hbar \omega_{j}}{K_{B} T}}-1}+1\right) \frac{\hbar}{2 \omega_{j}}\right) \frac{\left(\omega-\omega_{j}\right)^{4} V^{2}}{(4 \pi)^{2} c^{4}}\left|\vec{e}_{s} \cdot \frac{d \vec{\chi}}{d Q_{j}} \cdot \vec{e}_{i}\right|^{2} \\
\left(\frac{d \sigma}{d \Omega}\right)_{\text {anti-Stokes }}=\left(\frac{1}{e^{\frac{\hbar \omega_{j}}{K_{B} T}}-1} \frac{\hbar}{2 \omega_{j}}\right) \frac{\left(\omega+\omega_{j}\right)^{4} V^{2}}{(4 \pi)^{2} c^{4}}\left|\vec{e}_{s} \cdot \frac{d \vec{\chi}}{d Q_{j}} \cdot \vec{e}_{i}\right|^{2}
\end{gathered}
$$

De estas dos ecuaciones puede extraerse la relación existente entre las secciones eficaces para los eventos Stokes y anti-Stokes, que es equivalente a la relación de intensidades entre ambos tipos de radiación Raman [19]:

$$
\frac{I_{\text {anti-Stokes }}}{I_{\text {Stokes }}}=\left(\frac{\omega+\omega_{j}}{\omega-\omega_{j}}\right)^{4} e^{-\frac{\hbar \omega_{j}}{K_{B} T}} \approx e^{-\frac{\hbar \omega_{j}}{K_{B} T}}
$$

Esta relación muestra como la intensidad Raman anti-Stokes es mucho más débil que la intensidad Stokes a la temperatura común de trabajo en el laboratorio, $\left(\left(\frac{I_{\text {anti-Stokes }}}{I_{\text {Stokes }}}\right)_{300 \mathrm{~K}} \approx 0,1\right)$, y por tanto la radiación Stokes es la más adecuada para la caracterización de la muestra, sobre todo en estructuras con un bajo volumen de scattering como pueden ser los NWs en determinadas situaciones. Esta relación entre las intensidades Stokes y anti-Stokes puede ser utilizada para estimar la temperatura a la que se encuentra la muestra analizada, pero en muestras con una baja sección eficaz, como pueden ser los NWs semiconductores, resulta en medidas muy ruidosas salvo que la temperatura de la muestra sea muy elevada. Por otra parte, existen limitaciones en los equipos de medida que pueden impedir la adquisición de ambas intensidades Raman simultáneamente.

\section{Tensor Raman y reglas de selección}

El término $\frac{d \vec{\chi}}{d Q_{j}}$ que aparece en la ecuación 1.12 se denomina tensor Raman. Con independencia de la magnitud del campo eléctrico en el interior del NW, o de la intensidad del haz electromagnético incidente en la muestra, si el tensor Raman es nulo no se obtiene ninguna señal mensurable de la muestra; por tanto el tensor Raman determina que modos de vibración son activos en el cristal en espectroscopia 
Raman. Los tensores Raman para cada uno de los 32 grupos de simetría puntual fueron derivados en su momento por Loudon [20], obteniéndose en concreto para el silicio tres tensores Raman distintos:

$$
\left(\frac{d \vec{\chi}}{d Q_{j}}\right)_{x}=\left(\begin{array}{ccc}
0 & 0 & 0 \\
0 & 0 & d \\
0 & d & 0
\end{array}\right) ;\left(\frac{d \vec{\chi}}{d Q_{j}}\right)_{y}=\left(\begin{array}{ccc}
0 & 0 & d \\
0 & 0 & 0 \\
d & 0 & 0
\end{array}\right) ;\left(\frac{d \vec{\chi}}{d Q_{j}}\right)_{z}=\left(\begin{array}{ccc}
0 & d & 0 \\
d & 0 & 0 \\
0 & 0 & 0
\end{array}\right)
$$

De estas matrices se deduce que en ausencia de tensiones, los tres modos de vibración activos en el silicio están degenerados, apareciendo todos ellos a la misma frecuencia; en concreto $\omega_{j}=520,7 \mathrm{~cm}^{-1}$ para el silicio cristalino.

Por otro lado, en la ecuación 1.12 se observa como el tensor Raman va acompañado de los vectores unitarios que describen la polarización incidente y emitida. Esto implica que en muestras de silicio con superficies perfectamente orientadas en una determinada dirección cristalina, la combinación de estos vectores con los tensores Raman correspondientes resultan en una modulación de la intensidad Raman emitida por la muestra en función de la polarización de la luz incidente [21, 22]. Sin embargo las superficies de los NWs de silicio no pueden considerarse orientadas, por lo que la intensidad Raman obtenida de los NWs puede tomarse como prácticamente independiente del termino $\left|\vec{e}_{s} \cdot \frac{d \vec{\chi}}{d Q_{j}} \cdot \vec{e}_{i}\right|^{2}$ de la ecuación 1.13.

\subsubsection{Scattering Raman desde la interpretación de la mecánica cuántica}

El tratamiento clásico realizado en el apartado anterior no es suficiente para justificar algunas propiedades del scattering Raman, como por ejemplo el scattering Raman resonante, por ello es necesario analizar el proceso desde una descripción microscópica de cada evento de scattering.

Desde el punto de vista de la mecánica cuántica, en cada evento de scattering Raman intervienen tres jugadores, un fotón incidente de frecuencia $\omega$, un fonón de frecuencia $\omega_{k}$ y un fotón de frecuencia $\omega_{j}[23]$. El fotón incidente de frecuencia $\omega$ excita el material semiconductor de un estado inicial $\mid m>$ a un estado intermedio $\mid n>$, que puede ser un par electrón-hueco, tanto virtual como real, describiéndose 


\section{INTRODUCCIÓN}

el proceso a través del Hamiltoniano $H_{1}$. Desde este estado intermedio el material se relaja emitiendo (Stokes) o absorbiendo (anti-Stokes) un fonón de frecuencia $\omega_{k}$, pasando a estar en otro estado intermedio $\mid l>$. Este proceso se describe mediante el Hamiltoniano $\mathrm{H}_{2}$. Finalmente el material se relaja desde este segundo intermedio mediante una recombinación radiativa, emitiendo en el proceso un fotón de frecuencia $\omega_{j}$.

En este proceso los estados inicial y final no difieren, y por tanto ni se gana ni

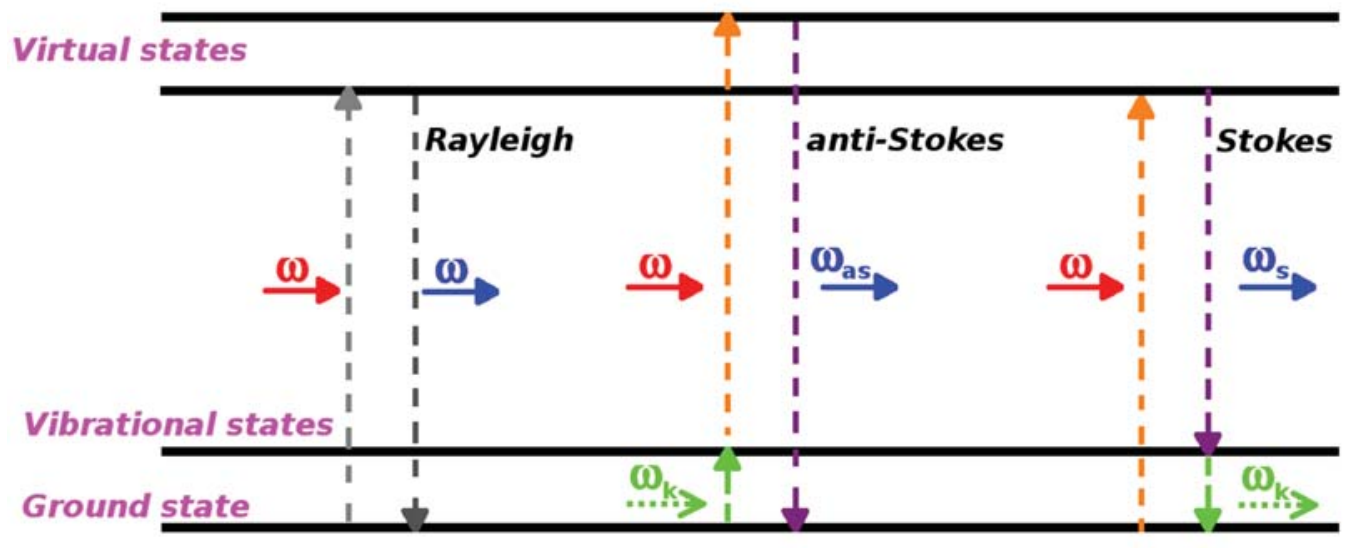

Figura 1.1: Diagrama de niveles de energía para los procesos de scattering Rayleigh, anti-Stokes y Stokes.

se pierde energía en el proceso. Por tanto, la diferencia entre las energías de los fotones incidente y Raman tiene que ser idéntica a la energía del fonón creado/aniquilado en proceso:

$$
\hbar \omega-\hbar \omega_{j}= \pm \hbar \omega_{k}
$$

Además de la conservación de la energía, en el scattering Raman de primer orden también se conserva el momento total, y por tanto:

$$
\hbar \vec{k}-\hbar \overrightarrow{k_{j}}= \pm \hbar \overrightarrow{q_{k}}
$$

Donde $\vec{k}$ es el vector de onda de la luz incidente y $\overrightarrow{q_{k}}$ el vector de onda del fonón implicado en el proceso. El valor máximo de $\overrightarrow{q_{k}}$ se alcanza en límite en el que el vector de onda del fotón Raman es igual en magnitud y opuesto en dirección al vector de onda del fotón incidente (condición de Backscattering), y por tanto 


\subsection{Descripción del equipo Raman}

$q_{k}<\frac{4 \pi}{\lambda}[7]$. Esto implica que para longitudes de onda superiores a $300 \mathrm{~nm}$ el valor de $q_{k}$ es mucho menor que la anchura de la zona de Brillouin, caracterizada por un valor máximo de $\pi / a$ siendo $a$ el parámetro de red. Esta condición limita los valores que puede tomar $q_{k}$ a la zona central de la primera zona de Brillouin, pudiéndose establecer la regla de selección para los fonones activos en Raman dada por:

$$
q_{k} \simeq 0
$$

La probabilidad de que suceda uno de estos eventos de scattering Raman puede describirse a través de [23]:

$$
P\left(\omega_{j}\right)=\frac{2 \pi}{\hbar} \sum_{n, l}\left|\frac{<m\left|H_{1}\right| l><l\left|H_{2}\right| n><n\left|H_{1}\right| m>}{\left(\hbar \omega-\left(E_{n}-E_{m}\right)\right)\left(\hbar \omega-\hbar \omega_{k}\left(E_{l}-E_{m}\right)\right)}\right|^{2} \delta\left(\hbar \omega-\hbar \omega_{k}-\hbar \omega_{j}\right)
$$

De esta expresión es simple deducir que si la frecuencia de los fotones incidentes es próxima a la energía de transición de los estados electrónicos del material, caracterizados por $\left(E_{n}-E_{m}\right)$ y $\left(E_{l}-E_{m}\right)$, la probabilidad de que sucedan eventos de scattering Raman aumenta dramáticamente, puesto que el denominador de 1.17 tiende a cero. A esta situación se la denomina scattering Raman resonante [23].

\subsection{Descripción del equipo Raman}

Los eventos de scattering Raman que se producen en un semiconductor iluminado por una fuente de luz, y que están caracterizados por la ecuación 1.17, tienen una frecuencia de aparición muy baja. Por tanto es necesario iluminar la muestra con luces muy intensas para obtener una señal mensurable. Esto se consigue iluminando la muestra con haces láser enfocados en áreas de iluminación muy reducidas. En nuestro caso se ha contado con dos fuentes de iluminación láser diferentes, un láser de He-Ne que emite en una longitud de onda de $632.8 \mathrm{~nm}$, y un láser de Nd:YaG doblado que emite en $532.8 \mathrm{~nm}$. Además de los haces láser como fuente de iluminación, un equipo Raman se compone principalmente de los siguientes componentes: un microscopio, un filtro que elimine la radiación Rayleigh, y un espectrómetro acoplado a un fotodetector (ver figura 2). 


\section{INTRODUCCIÓN}
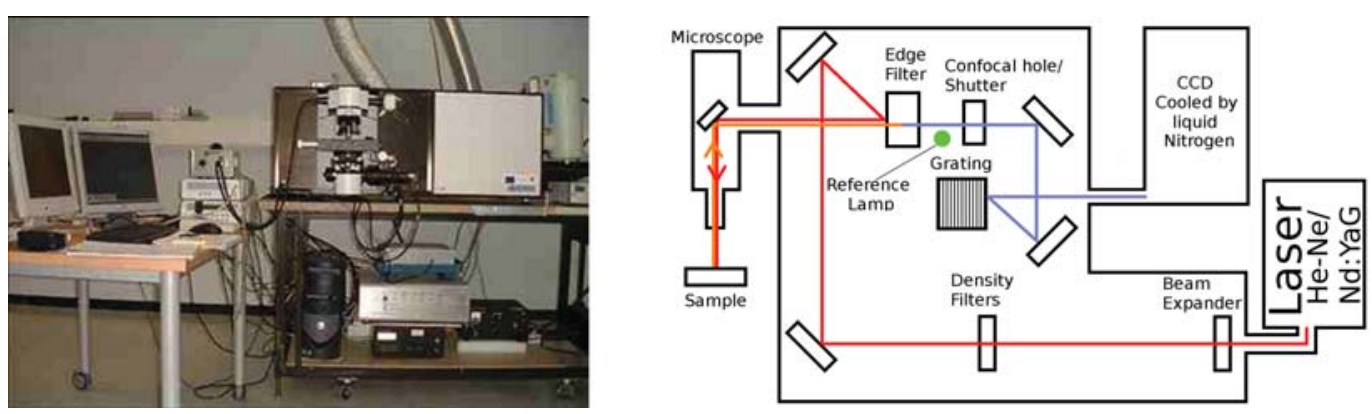

Figura 1.2: Equipo Raman Horiba Labram HR 800 de Yobin Ybon utilizado para la caracterización experimental de los NWs. A la derecha se muestra un esquema simplificado del equipo.

El microscopio se utiliza para focalizar la luz láser en los NWs y al mismo tiempo para recoger la emisión Raman procedente de la muestra iluminada. En todos los casos se ha utilizado un objetivo Olympus 100X con una apertura numérica de 0.95. Trabajar con este objetivo nos permite distinguir los NWs en la muestra, mientras que la luz láser se concentra en un área de iluminación cercano a $1 \mu \mathrm{m}$ para ambos láseres [24]. La resolución lateral del microscopio confocal (RL) configurado con este objetivo está comprendida entre los valores teóricos dados por el criterio de Rayleigh y el óptimo para microscopia confocal [24, 25]:

$$
\frac{0,4 \lambda}{N A}<R L<\frac{0,62 \lambda}{N A}
$$

De este modo, la resolución lateral para ambos láseres está comprendida entre algo menos de $300 \mathrm{~nm}$ y algo más de $400 \mathrm{~nm}$. Este valor de RL es importante para establecer una separación mínima entre puntos de adquisición cuando se realicé un análisis composicional en NWs de silicio-germanio en el capítulo 4; para asegurar que se iluminan zonas diferentes del NW, al menos se tendrán que separar los puntos de adquisición a lo largo del NW una distancia RL.

La radiación Rayleigh que proviene del semiconductor iluminado por un haz láser puede ser $10^{7}$ veces más intensa que la radiación Raman Stokes [14, 20]. Por otra parte, las energías de los fonones activos en la mayoría de los semiconductores son normalmente bajas, lo que implica que las frecuencias a las que aparecen los pi- 


\subsection{Descripción del equipo Raman}

cos Raman están muy próximas a la zona dominada por la radiación Rayleigh. Por tanto, y dada la enorme diferencia en la magnitud de la intensidad entre la emisión Raman y Rayleigh, es necesario un sistema capaz de discriminar y filtrar de forma eficiente la radiación Rayleigh procedente de la muestra. Para este propósito, en los espectrómetros Raman modernos se utilizan filtros notch o filtros pasabanda. Estos filtros reflejan toda la luz que tiene la misma frecuencia que el láser, mientras que permiten el paso de la luz con una frecuencia ligeramente diferente. Antiguamente se utilizaba para esto mismo sistemas basados en monocromadores, pero estos sistemas tienen la desventaja de limitar más la intensidad final que llega al detector que los sistemas basados en filtros modernos. Por su parte, los filtros notch tienen la ventaja de que permiten medir simultáneamente la radiación Raman Stokes y la anti-Stokes, mientras que los filtros pasabanda son específicos para cada una de ellas. Sin embargo, los filtros de pasabanda no se degradan tan rápidamente como los notch, por lo que son una opción más económica y estable en el tiempo. En concreto el equipo Raman con el que se ha trabajado incorpora filtros pasabanda configurados para la radiación Raman Stokes. Debido a esta configuración se ha trabajado siempre sobre la radiación Raman Stokes procedente de los NWs.

Por último, la luz filtrada procedente de la muestra se refleja en una rejilla antes de ser encaminada al CCD. Dependiendo del número de lineas por milímetro de esta rejilla de difracción obtendremos una mayor o menor resolución en frecuencia del espectro Raman. El equipo utilizado dispone de dos redes de difracción diferentes, una de 1200 lineas/mm y otra de 2400 lineas/mm. Para muestras con un señal Raman muy débil, como en principio pueden ser los NWs de silicio, es mejor utilizar la rejilla con menor densidad de lineas, puesto que la intensidad del espectro Raman es mayor en estas condiciones; sin embargo, se ha utilizado también la rejilla de 2400 lineas/mm para tener una mayor resolución en frecuencia, y por tanto un mayor detalle, de los picos que aparecen en el espectro Raman de los NWs. Finalmente, la luz procedente de la rejilla de difracción se focaliza en un array de CCD Symphony de Horiba, consistente en 1024x256 pixeles y enfriado con nitrógeno liquido por debajo de $130 \mathrm{~K}$ para mejorar la relación señal/ruido. Los espectros adquiridos con este equipo han sido tratados con el programa LabSpec en su versión 5.45. Este programa permite un completo tratamiento de los es- 


\section{INTRODUCCIÓN}

pectros Raman y el ajuste de los picos mediante funciones Gaussians, Lorentzianas o mezcla de ambas, tanto simétricas como asimétricas.

Por otro lado, para eliminar los efectos externos en las medidas, todo el equipo se encuentra sobre una mesa neumática Newport, de modo que se eliminan las posibles vibraciones de la estructura. Además el equipos se encuentra en una sala climatizada para mantener las variaciones de temperatura controladas.

\subsection{Microscopio electrónico}

Para la determinación de las características geométricas de los NWs analizados por espectroscopia Raman se ha recurrido a una caracterización por microscopia electrónica (SEM). La resolución de los microscopios ópticos está limitada por la longitud de onda de los fotones incidentes a través de la ecuación 1.18. Por tanto, cuanto menor es la longitud de onda de la luz incidente mayor resolución puede obtenerse. Esta limitación en la resolución se puede superar con la microscopia electrónica, donde se utilizan electrones en vez de utilizar fotones para obtener información de las muestras. A través de la dualidad onda-corpusculo propuesta por de Broglie, es conocido que los electrones tienen una longitud de onda asociada a su comportamiento ondulatorio. Esta longitud de onda está relacionada con el momento de los electrones a través de $p=\frac{\hbar}{\lambda}$ [26]. Por otro lado el momento de los electrones que son acelerados por una diferencia de potencial $V$ está dado por:

$$
p^{2}=2 m_{0} e V\left(1+\frac{e V}{2 m_{0} c^{2}}\right)
$$

Igualando estas expresiones para los momentos se puede encontrar una expresión para la longitud de onda de los electrones acelerados por un potencial $V$, encontrándose que la longitud de onda asociada a los electrones acelerados por voltajes del orden de los KV es varios ordenes de magnitud más pequeña que la de los fotones de la luz visible. Dado que resolución del microscopio depende fundamentalmente del tamaño del spot, y este parámetro depende de la longitud de onda de las partículas utilizadas (fotones, electrones, etc...), los microscopios basados en electrones tienen un poder de resolución teórico varios ordenes de magnitud superior a los microscopios ópticos $\left(i 10^{5}\right)$. [27] 
El tamaño del spot del microscopio electrónico no solo depende de la longitud de

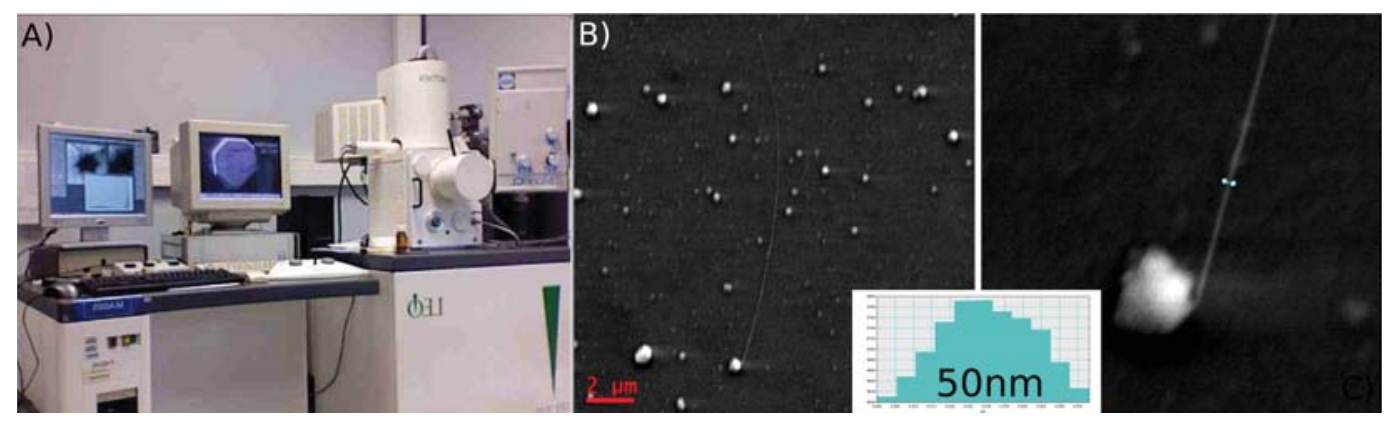

Figura 1.3: A) Equipo de microscopia electrónica LEO 1530 VP SEM utilizado para la caracterización experimental de los NWs. B) Imagen SEM de un NW de silicio adquirida a $5 \mathrm{KV}$ y $12500 \mathrm{X}$ aumentos. C) Imagen SEM a $5 \mathrm{KV}$ y $75000 \mathrm{X}$ aumentos del mismo NW.

onda de los electrones, y por tanto del voltaje utilizado para su aceleración, sino que también depende de la lente condensadora que controla el ángulo de convergencia del haz de electrones y de la apertura de objetivo. Debido a estos elementos, la resolución real de los microscopios electrónicos no es tan alta como cabría esperar por la pequeña longitud de onda de los electrones; la resolución teórica del SEM es del orden de $0.5 \mathrm{~nm}$ a $30 \mathrm{KV}$, dos ordenes de magnitud por encima del limite teórico marcado por la longitud de onda de los electrones.

El equipo SEM utilizado para la caracterización de los NWs es un LEO 1530 VP SEM, este equipo tiene una capacidad de aumento real de más de $100000 \mathrm{X}$, y una resolución real cercana a $1 \mathrm{~nm}$ a $20 \mathrm{kV}$. Sin embargo, para el propósito de esta investigación no son necesarias resoluciones tan elevadas. La caracterización SEM se realiza con el propósito de conocer la disposición espacial de los NWs analizados por Raman, su geometría y sus características superficiales; i.e. si es un NW se encuentra perfectamente aislado o tiene NWs adyacentes, determinar cual es su longitud y diámetro, o distinguir si sus superficies son limpias o irregulares.

Para este propósito es preferible trabajar con kilovoltages relativamente bajos, ya que no son necesarias unas resoluciones muy elevadas y puesto que la longitud de penetración es menor se obtiene una mayor información de la superficie de los NWs [28], permitiendo además una mejor determinación del diámetro real de los NWs. Los resultados típicos de esta caracterización SEM se muestran en la figura 


\section{INTRODUCCIÓN}

1.3, donde se observa la imagen SEM tomada en una muestra con NWs de silicio depositados en aluminio. El potencial utilizado es relativamente bajo, $5 \mathrm{KV}$, y sin embargo la imagen adquirida revela perfectamente que el NW está aislado, permitiendo además obtener el diámetro del mismo de forma precisa.

\subsection{Otras consideraciones}

Los NWs analizados experimentalmente en el transcurso de esta tesis no han sido crecidos en el propio grupo de investigación, sino que proceden de diferentes colaboradores que nos los han cedido amablemente para su caracterización.

Los NWs de silicio proceden en su mayoría de nuestros colaboradores del departamento de Tecnología Electrónica de la universidad Universidad Politécnica de Madrid, pero también se han analizado NWs de silicio procedentes de nuestros colaboradores del Institut dÉlectronique et des Télécommunications de la universidad de Rennes. Por su parte, todos los NWs de silicio-germanio analizados proceden del grupo de la Universidad Politécnica de Madrid.

En todo caso los NWs se han sido crecidos por el método Vapor-liquido-sólido (VLS) en reactores LPCVD (low pressure chemical vapor deposition). Dado que no hemos sido nosotros los encargados del crecimiento de los NWs no se va a entrar en detalle sobre los mecanismos de crecimiento de los mismos, pudiéndose consultar todo lo referente a este proceso en la referencia [29].

Por otra parte, de los NWs de silicio y silicio-germanio procedentes del grupo de Madrid se dispone de información sobre su cristalinidad y composición. Esta información procede de una caracterización mediante TEM+EDX llevado a cabo previamente por el grupo de física de la Universidad Carlos III de Madrid. Esta caracterización se ha realizado en las mismas muestras que posteriormente se han utilizado en la caracterización Raman, permitiéndonos comparar algunos resultados obtenidos con ambas técnicas experimentales. 


\subsection{Bibliografía}

[1] Lu W, Lieber C. M. Semiconductor Nanowires, J. Phys. D: Appl. Phys. 2006;39, 387.

[2] Hochbaum A. I, Yang P. Semiconductor Nanowires for Energy Conversion, Chem. Rev. 2010;110, 527.

[3] Grabert H, Devoret M. H. Single Charge Tunneling: Coulomb Blockade Phenomena in Nanoestructures, 1992, Plenum, New York. [4] Li D, Wu Y, Kim P, Shi L, Yang P, Majumdar A. Thermal conductivity of individual silicon nanowires. Appl.Phys.Lett. 2003;83, 2934. [5] Cao L, White J. S, Park J. S, Schuller J. A, Clemens B. M, Brongersma M. L. Engineering light absorption in semiconductor nanowire devices. Nature mater. 2009;8, 643

[6] Hochbaum A.I, Chen R, Delgado R.D, Liang W, Garnett E.C, Najarian M, Majumdar A, Yang P. Enhanced thermoelectric performance of rough silicon nanowires. Nature, 2008;451, 163

[7] Doerk G. S, Carraro C, Maboudian R. Raman Spectroscopy for Characterization of Semiconductor Nanowires, en Raman Spectroscopy for Nanomaterials Characterization, Ch 17, 2012, Springer-Verlag, Heilderberg.

[8] Scheel H, Reich S, Ferrari A. C, Cantoro M, Colli A, Thomsen C. Raman scattering on silicon nanowires: The thermal conductivity of the environment determines the optical phonon frequency. Appl. Phys. Lett. 2006;88, 233114

[9] Bhattacharya S, Samui S. Phonon confinement in oxide-coated silicon nanowires. Appl. Phys. Lett. 2004;84, 1564

[10] Soini M, Zardo I, Uccelli E, Funk S, Koblmuller G, Fontcuberta A, Abstreiter G. Thermal conductivity of GaAs nanowires studied by micro-Raman spectroscopy combined with laser heating. Appl. Phys. Lett. 2010;97, 263107

[11] Jalilian R, Sumanasekera G. U, Chandrasekharan H, Sunkara M. K. Phonon confinement and laser heating effects in Germanium nanowires. Phys. Rev. B 2006;74, 155421

[12] Smekal A. Zur Quantentheorie der Dispersion, Naturwissenschaften, 1923;11, 873.

[13] Raman C.V, Krishnan K. S. A New Tipe of Secondary Radiation, Nature 1928;121, 501. 
[14] Cardona M, Güthertodt G. Light Scattering in Solids, en Topics in Applied Physics, Vol 50, 1982, Springer-Verlag, Heilderberg.

[15] Long D. Raman Spectroscopy, 1977, McGraw-Hill, New York.

[16] Derek L. A.The Raman Effect: A Unified Treatment of the Theory of Raman Scattering by Molecules, Ch 3, John Wiley \& Sons, Chichester, 2002.

[17] Nafie L. A. Theory of Raman Scattering, Handbook of Raman Spectroscopy, Edit Lewis, R. I and Edwards H. G. M.Mercel Dekker, New York, 2001.

[18] Bauer M. Raman spectroscopy of laser induced material alterations, Tesis, 2010, Ludwig-Maximilians-Universitat, Munich.

[19] Hart T. R, Aggarwal R. L, Lax B. Temperature Dependence of Raman Scattering in Silicon, Phys. Rev. B. 1970;1, 638.

[20] Loudon R. Raman Effect in Cristals, Adv. in Phys. 1964;13, 423.

[21] De Wolf I. Micro-Raman spectroscopy to study local mechanical stress in silicon integrated circuits, Semicond. Sci. Technol. 1996;11, 139.

[22] Nakashima S, Hangyo M. Characterization of Semiconductor Materials by Raman Microprobe, IEEE Journal of Quantum Electronics, 1989;25, 965

[23] Yu P. Y, Cardona M. Fundamentals of Semiconductors, Physics and Material Properties, 1996, Springer-Verlag, Heilderberg.

[24] Jiménez J, de Wolf I, Landesman J. P. Microprobe Characterization of Semiconductors; Optoelectronic properties of semiconductors and superlattices. Ch 2 , Vol 17, ( ed. by J. Jiménez, Taylor and Francis, New York 2002)

[25] Spring K. R. Resolution and Contrast in Confocal Microscopy. [26] Schrödinger E. An Undulatory Theory of the Mechanics of Atoms and Molecules, Phys. Rev. 1926;28, 1049.

[27] Williams D. B, Barry C. CarterTransmission Electron Microscopy: A Textbook for Materials Science,1996, Vol 1, Springer Science, New York.

[28] Kanaya K, Okayama, S, Penetration and Energy-Loss Theory of electrons in Solid Targets, J. Phys. D: Appl. Phys., 1972:5, 43.

[29] Wang N, Cai Y, Zhang R. Q. Growth of Nanowires, Materials Science and Engineering, 2008;60, 1 . 
The most beautiful thing we can experience is the mysterious. It is the source of all true art and all science. He to whom this emotion is a stranger, who can no longer pause to wonder and stand rapt in awe, is as good as dead: his eyes are closed. Albert Einstein

\section{Transporte térmico en NWs semiconductores}

\subsection{Introducción}

Desde finales de la década de los 90, la investigación en NWs semiconductores está en continua expansión debido al enorme número de potenciales aplicaciones en las que estas estructuras unidimensionales desempeñan un papel principal. Esta popularidad se ha traducido en una enorme cantidad de publicaciones científicas que giran entorno a sus diferentes propiedades y utilidades. Los NWs semiconductores son piezas claves en el desarrollo de nuevos dispositivos de escala reducida, como por ejemplo nano-FET's, nano-interconectores, dispositivos nanoestructurados, diodos láser de orientación vertical en nanoescala, conversores termoeléctricos, dispositivos fotovoltaicos de alto rendimiento o sensores nanométri$\cos [1,2,3,4,5]$.

Dentro de las investigaciones llevadas a cabo en NWs, el transporte térmico en estas estructuras casi unidimensionales es uno de lo temas que más atención está atrayendo. Dada la reducida dimensión lateral que presentan los NWs, incluso la disipación de pequeñas cantidades de energía suponen unas densidades de energía a 


\section{TRANSPORTE TÉRMICO EN NWS SEMICONDUCTORES}

disipar enormes. Este efecto es muy relevante en dispositivos que operan bajo grandes densidades de corriente, en los cuales se induce un calentamiento Joule nada despreciable. Por lo tanto, el control de la conductividad térmica es de vital importancia para controlar la temperatura de las partes activas de estos dispositivos [6]. Por otro lado, los materiales semiconductores normalmente son buenos conductores eléctricos y térmicos, lo que limita su utilización como dispositivos de conversión termoeléctrica; sin embargo, se ha demostrado experimentalmente que la conductividad térmica intrínseca de los NWs semiconductores es mucho menor que la conductividad térmica equivalente para el semiconductor volúmico, pudiendo incluso comportase como aislantes térmicos mediante la modificación de la superficie de los NWs [2]. La reducción en la conductividad térmica no lleva aparejada una perdida sustancial en las cualidades de transporte eléctrico del NW. Dado que la eficiencia en la conversión termoeléctrica está caracterizada por la figura de merito $[2,7,8,9]$ :

$$
Z T=S^{2} T / \rho\left(\kappa_{e}+\kappa_{l}\right)
$$

Ésta depende del coeficiente Seebeck $(Z T)$,la temperatura $(T)$, la resistencia eléctrica $(\rho)$ y la conductividad térmica dada como $\left(\kappa_{e}+\kappa_{l}\right)$, donde la primera componente se refiere a la conductividad térmica electrónica y la segunda es la intrínseca del NW debida a los fonones de la red, de donde se deduce que la reducción en la conductividad térmica de la red en los NWs semiconductores conlleva a un aumento en la figura de merito, ya que está desacoplada de las magnitudes dependientes de la componente electrónica. Este efecto convierte a los dispositivos construidos con NWs semiconductores en buenos candidatos para la construcción de dispositivos termoeléctricos baratos de alto rendimiento. $[2,7,8,9]$

La determinación experimental de la conductividad térmica en nanoescala es un proceso muy complejo y laborioso $[11,12,13]$, por lo que existen muy pocas medidas contrastadas en la literatura. Las primeras medidas de transporte térmico en NWs semiconductores se llevaron a cabo por D. Li et al. [11,13], quienes midieron la conductividad térmica de unos pocos nanohilos individuales crecidos por el método VLS. Para medir la conductividad térmica de estructuras unidimensionales utilizaron un microdispositivo específicamente desarrollado por S.Li et al., el cual está basado en la idea del puente térmico [10]. Éste consiste en un NW suspendi- 
do entre dos placas de platino, una de las cuales se calienta mientras que la otra actúa de termómetro. Para garantizar una buena medida, la unión entre el NW y las placas de platino tienen que tener una resistencia térmica idealmente nula, lo que dificulta mucho la medida, ya que al manejar una sección nanométrica es muy delicado realizar una soldadura que no introduzca ruido en la medida[10, 12] .

En estos resultados experimentales se observa una fuerte reducción de la conductividad térmica de los NWs de silicio en comparación con la conductividad térmica del silicio volúmico (Figura 2.1-A). Además se descubrió que la conductividad térmica de los NWs de Si tienen una fuerte dependencia con el diámetro del NW; cuanto menor es el diámetro, menor es la conductividad térmica del NW. Por tanto, la determinación de la conductividad térmica en estas estructuras es más compleja que en macroescala, dado que es necesario determinar la conductividad térmica de un NW de un diámetro especifico. Por otro lado, los datos experimentales revelan un cambio de comportamiento de la conductividad térmica en función de la temperatura cuando el diámetro de los NWs de silicio traspasan la barrera de los 25 $\mathrm{nm}$; los datos experimentales para un NW de $22 \mathrm{~nm}$, para los cuales los efectos de confinamiento fonónico no pueden ser ignorados [15], muestran una dependencia de la conductividad térmica pseudo-lineal con la temperatura, mientras que para diámetros mayores, la forma de la conductividad térmica muestra una dependencia similar a la que sigue el Silicio volúmico (Figura 2.1-B).

Utilizando el mismo dispositivo desarrollado por S. Li et al., diversos autores han determinado la conductividad térmica de NWs de distintos materiales semiconductores $[13,16,17,18,19,20]$. Todos los NWs medidos con esta técnica experimental, y con independencia del material del que los NWs están constituidos, muestran una fuerte reducción de la conductividad térmica frente a la conductividad térmica del semiconductor volúmico correspondiente, revelando por tanto que la conductividad térmica de los semiconductores unidimensionales está fuertemente influenciada por las dimensiones de la estructura.

Posteriormente, M.C. Wingert et al. llevaron a cabo la determinación experimental en NWs de germanio [20], mostrando que, además de la consabida dependencia de la conductividad térmica con el diámetro, el cambio en el comportamiento de la conductividad térmica a un régimen pseudo-lineal con la temperatura por debajo de un determinado diámetro, observado en NWs de Si, también se reproducía 

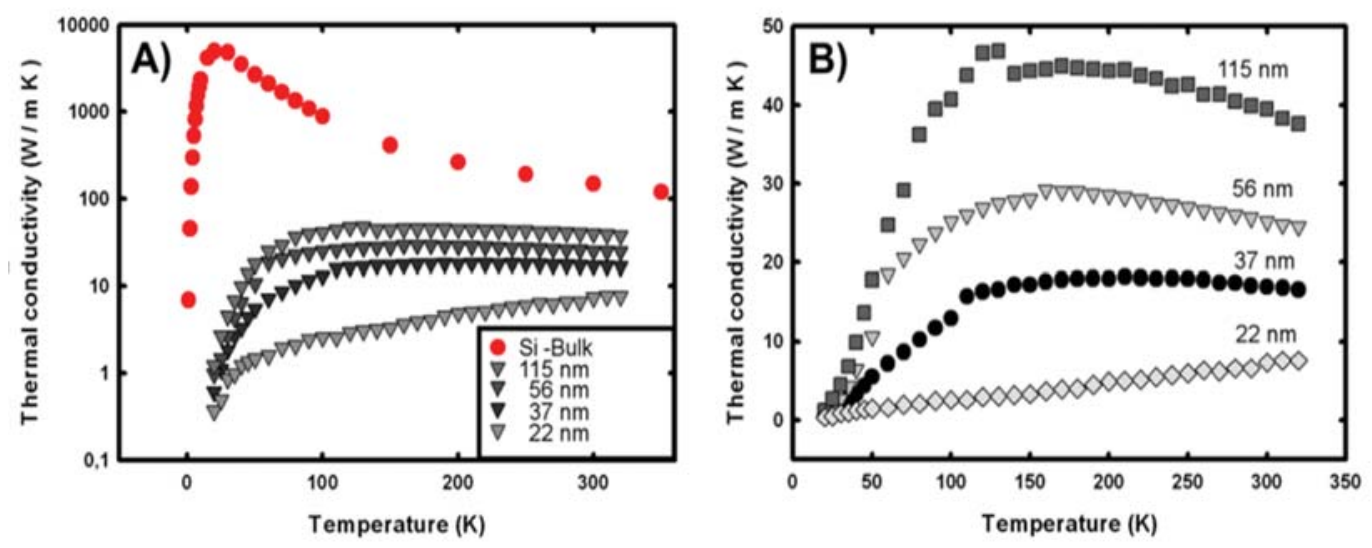

Figura 2.1: Conductividad térmica experimental en función de la temperatura para el Silicio volúmico [14] y NWs de Silicio con distintos diámetros [11] A), la conductividad térmica está en escala logarítmica. Conductividad térmica de los NWs de silicio en una escala expandida (B)

en NWs de germanio para diámetros menores a $20 \mathrm{~nm}$ (Figura 2.2). Por lo tanto, este fenómeno puede considerarse como una propiedad intrínseca en los NWs de dimensiones reducidas en las que los efectos de confinamiento fonónico son relevantes; y por tanto, el transporte térmico debido a dichos fonones deja de comportarse como en las estructuras volúmicas, ó en aquellos reducida dimensión con confinamiento fonónico despreciable.

\section{Efecto de la morfología superficial de los NWs en la conductivi- dad térmica}

La conductividad térmica en NWs semiconductores no se ve limitada únicamente por las dimensiones de la estructura, sino que hay otros factores que pueden llegar a ser incluso más relevantes. A. Hochbaum et al. demostraron experimentalmente como en NWs de silicio construidos de tal modo que su superficie presentase una rugosidad elevada, la conductividad térmica se reducía en mayor medida que en NWs con superficies lisas como las que presentaban los NWs utilizados por D.Li et al. [2]. Utilizando la misma técnica de medida que D. Li et al., pero en NWs de Si 


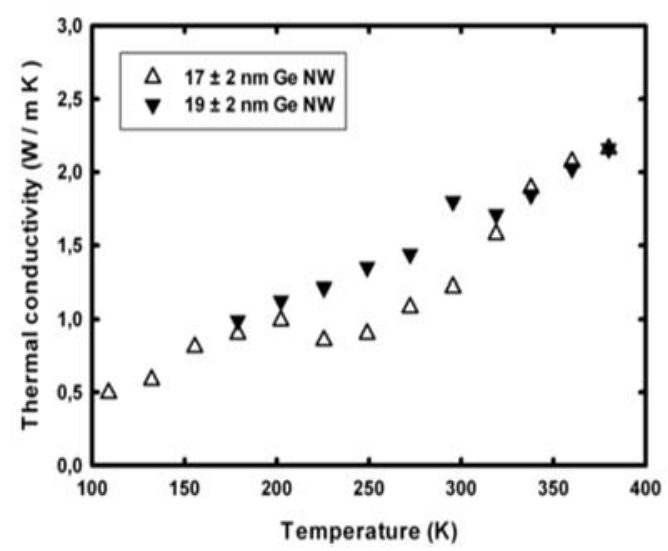

Figura 2.2: Conductividad térmica de dos NWs de germanio por debajo del límite de confinamiento cuántico, puede observarse como aparece una dependencia pseudolineal con la temperatura como la observada en NWs de silicio de similares dimensiones $[20]$

crecidos mediante un método diferente con el cual obtuvo NWs con unas rugosidades con una altura media ( $R M S$ o $\eta$ ) cercana a $5 \mathrm{~nm}$, frente al valor cercano a $1 \mathrm{~nm}$ de los NWs considerados como lisos, además la separación entre rugosidades (longitud de correlación) se encontraba en el orden de las decenas de nanómetros. El efecto introducido por la rugosidad en la disminución de la conductividad térmica de los NWs de Si puede verse en la Figura 2.3, donde se aprecia como la conductividad térmica de dos NWs de Si de idéntico diámetro no coincide, siendo la conductividad del NW rugoso 5 veces menor. Cabe recordar que la única diferencia estriba en las características de superficie de cada uno de ellos, ya que ambos mantienen similares propiedades geométricas y de cristalinidad (orientación, impurezas...).

En la misma linea, Y. Park et al. [21] llevaron a cabo un estudio similar en NWs con superficies rugosas, pero estos crecidos por el método VLS y con unos valores de $r m s$ aún mayores $(r m s>10 n m)$, por lo que cabría esperar una mayor reducción en la conductividad térmica que la observada por Hochbaum et al.[2]. Sin embargo, la conductividad térmica de estos NWs rugosos no era tan baja como los valores alcanzados en la referencia [2]. De este modo se deduce que la conductividad térmica no solo depende del tamaño medio de la rugosidad característica de 


\section{TRANSPORTE TÉRMICO EN NWS SEMICONDUCTORES}

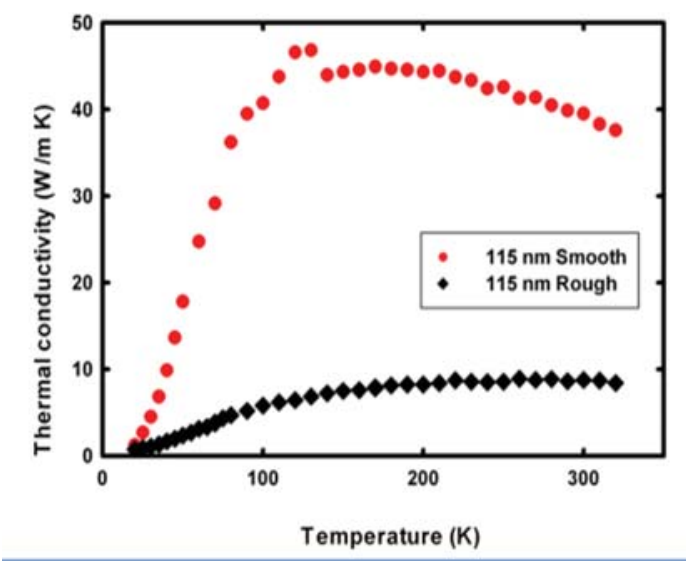

Figura 2.3: Conductividades térmicas de dos NWs de Si de iguales diámetros, 115 $\mathrm{nm}$, siendo uno de ellos de superficie rugosa [2] y otro de superficie lisa [11]

cada NW, sino que hay que tener en cuenta otros factores que limitan el transporte térmico en estas estructuras.

Corroborando este resultado, J. Lim et al. [22] demostró experimentalmente como la combinación entre el tamaño medio de la rugosidad y la longitud de correlación, dada por la separación media entre estas rugosidades, determina la reducción de la conductividad térmica en NWs. Para llegar a esta conclusión, J. Lim et al. midieron experimentalmente la conductividad térmica de diversos NWs de silicio con diferentes características superficiales, encontrando que en NWs de diámetros y valores de $R M S$ equivalentes, la conductividad térmica era muy dependiente de la longitud de correlación de las rugosidades; para igual valor de $R M S$, una longitud de correlación más corta implica una menor conductividad térmica.

Esta dependencia de la conductividad térmica de los NWs semiconductores con la longitud de correlación de la rugosidad característica explica porque los NWs muy rugosos de la referencia [21] presentan una menor reducción en la conductividad térmica que los NWs menos rugosos medidos en [2]. Observando las imágenes SEM que aparecen en ambos trabajos [2,21], es evidente que la longitud de correlación de los NWs medidos en [21] es mucho mayor que la que se observa en [2], y por tanto el transporte térmico fonónico se ve menos afectado pese a la mayor rugosidad,lo que confiere un papel relevante a la longitud de correlación. 


\section{Efecto de la composición de los NWs en la conductividad térmica}

El estudio del transporte térmico en NWs fabricados a partir de una aleación de materiales requiere tener en cuenta el efecto de la composición en la conductividad térmica. H. Kim et al. determinaron experimentalmente como afecta esta variable a la conductividad térmica de NWs de $S i_{x} G e_{1-x}$ [23]. Incluso para NWs con una componente de germanio muy pequeña $(x \simeq 0,004)$, encontraron que la conductividad térmica se veía muy reducida en comparación con NWs equivalentes de silicio puro. Posteriormente, $\mathrm{H}$. Kim et al. combinaron el estudio de la composición con el efecto de la morfología superficial en NWs de $S i_{x} G e_{1-x}$, mostrando como combinando estos dos efectos, la conductividad térmica de los NWs de $S i_{x} G e_{1-x}$ podía reducirse a valores cercanos al límite amorfo del material [24], abriendo por tanto una puerta a la obtención de dispositivos con una conductividad térmica prediseñada, algo muy deseable para la construcción de dispositivos de alta efectividad termoeléctrica. Este resultado puede observarse en la figura 2.4, donde se representa la conductividad térmica experimental de dos NWs de superficie lisa, uno de silicio y otro de silicio-germanio con una cantidad de germanio de solo el 0.4 , pese a la pequeña cantidad de germanio y al mayor diámetro del NW aleado, la conductividad térmica es casi 5 veces menor que para el NW de silicio puro. En la misma figura también se representa la conductividad térmica experimental de dos NWs de silicio-germanio de diámetros cercanos a 200 nm e idéntica proporción de germanio, pero diferentes morfologías superficiales, apreciándose como el efecto de la rugosidad se suma al efecto introducido por la aleación a la hora de reducir la conductividad térmica.

\section{Modelos teóricos de la conductividad térmica de NWs semicon- ductores}

El transporte térmico en estructures de baja dimensionalidad, como son los NWs semiconductores, ha recibido mucha atención por parte la comunidad científica. Antes incluso de que los primeros resultados experimentales demostrasen el especial comportamiento de la conductividad térmica de estas estructuras, ya existían 


\section{TRANSPORTE TÉRMICO EN NWS SEMICONDUCTORES}

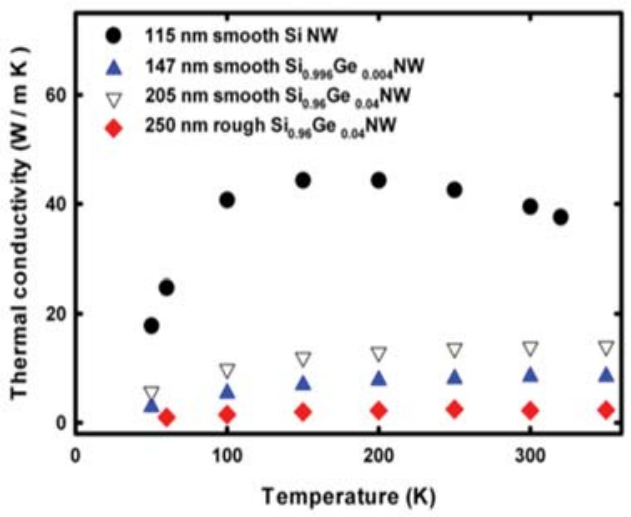

Figura 2.4: Comparación de la Conductividad térmica experimental de un NW liso de silicio y $115 \mathrm{~nm}$ (circulo negro)[11], frente a un NW liso de silicio-germanio con un 0.4 de germanio y $147 \mathrm{~nm}$ de diámetro (triángulos azules). También se compara la conductividad térmica de dos NWs de silicio-germanio de igual composición pero uno de ellos liso y de $205 \mathrm{~nm}$ de (triángulos blancos) y otro rugoso de $250 \mathrm{~nm}$ (rombos rojos)

algunos estudios teóricos que trataban este tema. A. Balandin et al. en un trabajo pionero sobre el efecto de la baja dimensionalidad en la conductividad térmica [25], realizó un análisis en un pozo cuántico semiconductor. Este primer modelo giraba entorno a la idea de que el confinamiento espacial implicaba una reducción de las velocidades de grupo de los fonones, y se calculaba la conductividad térmica mediante una versión reducida del modelo de Callaway [26] en la que se consideran únicamente los procesos de scattering resistivo dados por los procesos de umklapp, superficie e impurezas. -Más adelante y en este mismo capítulo se dará una completa descripción del formalismo de Callaway y de los términos de scattering implicados-. El estudio teórico realizado por Balandin et al. [25] reveló que la conductividad térmica de una nanoestructura de silicio es mucho más baja que la conductividad térmica del silicio volúmico. Poco después, el mismo grupo de investigación aplicó el formalismo anterior al estudio de la conductividad térmica de NWs de silicio [27], modificando para ello los términos utilizados para describir los diferentes eventos de scattering fonónico implicados en el transporte térmico, especialmente mediante el añadido del efecto de la rugosidad de la superficie, el cual se introducía a través de la probabilidad de los fonones de su- 
frir un evento de scattering especular o difusivo en la superficie del NW [28]. Con todas estas consideraciones, encontraron que la conductividad térmica de un NW de silicio de $20 \mathrm{~nm}$ de diámetro dependía enormemente de las condiciones tomadas para los eventos de scattering debidos a la superficie; pero, incluso en el caso de tomar el scattering de superficie más agresivo posible, la conductividad térmica calculada fue de $14 \mathrm{~W} / \mathrm{mK}$ a $300 \mathrm{~K}$, casi el doble que la conductividad térmica experimentalmente obtenida posteriormente en [11] para un NW liso de silicio de $22 \mathrm{~nm}$. También con anterioridad a las primeras medidas experimentales, A.Voltz et al. haciendo uso de una simulación de dinámica molecular en NWs de silicio de diámetros por debajo de $5 \mathrm{~nm}$, predijeron el mismo resultado de una acusada reducción de la conductividad térmica en semiconductores unidimensionales [29]. Con la llegada de las primeras medidas experimentales confirmando estos resultados se abrió la puerta al desarrollo de modelos teóricos desarrollados a través de ajustes a datos experimentales $[11,13]$. Como ya se ha mencionado anteriormente, las medidas experimentales evidencian una acusada dependencia con las propiedades geométricas de cada NW, como son diámetros y morfología de superficie; por lo tanto, la determinación experimental de la conductividad térmica se convierte en un trabajo titánico que debe hacerse casi para cada NW. Debido a esto, el desarrollo de un modelo capaz de predecir con precisión la conductividad térmica de NWs semiconductores es un tema de gran relevancia a la hora de diseñar dispositivos basados en estas estructuras, y por ello el desarrollo de estos modelos se ha convertido en un prolífico tema de investigación.

N. Mingo fue el primero que desarrolló un modelo capaz de ajustar razonablemente bien a los datos obtenidos para la conductividad térmica de los NWs lisos de silicio mostrados en [11]. Para ello utilizó un modelo basado en una modificación al modelo balístico de transporte de energía fonónico en un NW suspendido [30]. La modificación introducida consiste en añadir los eventos de scattering que sufren los fonones en un cristal real, y requiere calcular previamente las relaciones de dispersión completas de los fonones para cada NW. Los eventos de scattering considerados en este modelo son idénticos a los considerados en el modelo del grupo de Baladin [25, 27], pero a diferencia de éste, las expresiones para los eventos Umppklap y los debidos a impurezas no se construyen mediante expresiones exactas, sino que se introducen dos parámetros de ajuste que se fijan ajustando a los 


\section{TRANSPORTE TÉRMICO EN NWS SEMICONDUCTORES}

datos de la conductividad térmica para el Silicio volúmico, conservando su valor para los NWs de silicio. Desafortunadamente, este modelo infravalora el valor de la conductividad térmica con respecto a los datos experimentales, teniendo que recurrir a un parámetro de ajuste concerniente al término de scattering de superficie. Esto equivale a definir un diámetro efectivo bastante más grande que el diámetro nominal observado en el SEM para obtener un buen ajuste a los datos experimentales, lo que penaliza la utilización de este modelo para predecir conductividades térmicas de NWs no medidos. Por otro lado, este modelo no es capaz de ofrecer un ajuste aceptable al NW liso de silicio de $22 \mathrm{~nm}$ de diámetro, por lo que por debajo del confinamiento fonónico este modelo no es utilizable. Por otro lado, N. Mingo mostró como la utilización de un modelo basado en el formalismo de Callaway, como el utilizado por Baladin en [25,27], puede ofrecer un buen ajuste a los datos experimentales si se toman valores de la temperatura de Debye, o temperatura de corte, menores que las temperaturas de Debye para el material volúmico [30].

Los modelos recopilados hasta ahora solo consideran los procesos de scattering fonónico resistivos, lo que implica que la contribución de los procesos a tres fonones normales son ignorados. Sin embargo, es bien sabido que estos procesos contribuyen a la resistencia del transporte térmico a través del llamado mecanismo de Herring [32]. Y. Chen et al. fueron los primeros en incorporar este proceso de scattering en un modelo para la conductividad térmica basado en una simulación Monte Carlo para los eventos de scattering de fonones [33]. En esté formalismo se requieren dos parámetros de ajuste a los datos experimentales de la conductividad térmica del silicio volúmico, que luego se extrapolan al modelo para NWs. El ajuste a los datos experimentales obtenido por este método es muy bueno para temperaturas por debajo de $120 \mathrm{~K}$ y para diámetros mayores de $50 \mathrm{~nm}$, pero sobrestima la conductividad térmica para valores de temperatura más elevados y se aleja mucho de los valores experimentales para diámetros menores a $50 \mathrm{~nm}$, fallando completamente para NWs por debajo del limite de confinamiento fonónico $(D<25 \mathrm{~nm})$.

En otro intento de obtener un buen modelo para la conductividad térmica en NWs semiconductores, M. Huang et al. se centraron en desarrollar un completo tratamiento para los eventos de scattering de fonones en la superficie de los NWs [34], para posteriormente implementarlo en un modelo derivado del formalismo de Callaway como el empleado en [27]. Pese a contar con un tratamiento más completo 
del efecto de superficie sobre el transporte fonónico de energía, los resultados no responden demasiado bien a los datos experimentales para NWs lisos de silicio. Por otro lado, en este trabajo se deja patente que no solo las velocidades del sonido disminuyen al disminuir el diámetro, tal y como mostró Balandin et al en [25], sino que como N. Mingo intuía en [30], las temperaturas de corte de los NWs también deben ser menores que en sus homólogos volúmicos. Este resultado resulta de gran interés, jugando un papel principal posteriormente en la construcción de nuestro modelo para la conductividad térmica en NWs semiconductores.

Como puede observarse, todos los intentos de ajustar la conductividad térmica en NWs lisos de silicio descritos hasta este momento fallan estrepitosamente para NWs de diámetros menores a $25 \mathrm{~nm}$. L. Liang et al. [35] abordo este problema por medio de la relación entre la conductividad térmica a $300 \mathrm{~K}$ y la temperatura de fusión, tanto para NWs como para el silicio volúmico; el modelo incorpora el efecto del scattering de superficie en el transporte térmico a través de una relación exponencial dependiente del valor $R M S$ de la rugosidad superficial. Mediante este tratamiento simplificado es posible obtener una buena aproximación a la conductividad térmica a $300 \mathrm{~K}$ en nanocapas de diversos materiales semiconductores, y también para la conductividad térmica de los NWs lisos de silicio medidos en [11], incluso para el problemático NW de $22 \mathrm{~nm}$, para el cual los modelos anteriores no servían. El extraño comportamiento de la conductividad térmica para estos NWs de reducido diámetro ha sido estudiado por P. Murphy et al. en [36]; a diferencia de los trabajos anteriores, aquí se propone que en superficies fuertemente desordenadas, como puede sera la superficie de un NW, no todos los fonones sufren los mismos eventos de scattering, siendo estos eventos dependientes de la frecuencia de los fonones incidentes en la superficie. Basándose en esta hipótesis, P. Murphy et al. desarrollaron un modelo en el que el comportamiento de los fonones se puede separar en dos comportamientos diferentes distinguidos por la magnitud de su vector de onda en la dirección perpendicular al eje longitudinal del NW. El comportamiento es casi balístico para aquellos fonones con una componente transversal pequeña, mientras que los que tienen una componente transversal grande se encuentran en un régimen puramente difusivo en la superficie, con un camino libre medio (MFP) del orden del diámetro del NW. Bajo estas condiciones este modelo muestra que la conductividad térmica en NWs semiconductores de pequeño diámetro (i.e. $D<20 \mathrm{~nm}$ 


\section{TRANSPORTE TÉRMICO EN NWS SEMICONDUCTORES}

) tiene un comportamiento cuasi-lineal con la temperatura. Además muestra como el cambio de comportamiento entre la conocida dependencia de la conductividad térmica con la temperatura en escala macroscópica dada por $T^{3}$ y el régimen lineal de los NWs más finos se produce para un diámetro comprendido entre $25 \mathrm{~nm}$ y 20 $\mathrm{nm}$, tal y como las medidas experimentales muestran $[11,20]$.

Otro factor importante considerado por diversos autores es el papel jugado por los fonones ópticos en el transporte térmico de estas estructuras unidimensionales. Z. Tiang et al. [37] haciendo uso de las relaciones de scattering fonón-fonón descritas por A. Ward et al. [38], calcularon la contribución al transporte térmico de cada banda fonónica, mostrando que la contribución de los fonones ópticos puede representar cerca del $20 \%$ del total. Bajo la hipótesis difusiva para el scattering de superficie, y por tanto en el limite de Casimir [28], el modelo así desarrollado obtiene un buen ajuste a los datos experimentales para NWs lisos de silicio medidos en [11]. En un trabajo previo, M. Kazan et al. ya habían considerado el efecto de los fonones ópticos en el transporte térmico en NWs semiconductores [39], pero el mecanismo descrito era distinto, considerando en este caso el decaimiento de fonones ópticos en fonones acústicos, siendo estos últimos los responsables del transporte energético. Estos mismos autores ya habían mostrado que considerando este decaimiento óptico en fonones acústicos, y utilizando el formalismo de Callaway para el cálculo de la conductividad térmica, se podía obtener un mejor ajuste a los datos experimentales de la conductividad térmica del Germanio volúmico [40]. Cabe destacar que el formalismo de Callaway fue concebido bajo la aproximación acústica, teniendo únicamente en cuenta a los fonones acústicos en el transporte térmico [41, 42,43]; a pesar de esta omisión, el modelo de Callaway da un muy buen resultado a la hora de calcular la conductividad térmica en semiconductores, ya que la contribución debida a los procesos ópticos se puede incorporar tomando una mayor temperatura de corte (temperatura de Debye) [44]. Dado que Kazan et al. incorporan explícitamente la contribución óptica, las temperaturas de corte que toman para sus cálculos son menores que las tomadas tradicionalmente [41, 42], estas consideraciones sobre temperaturas de corte se verán con mas detalle posteriormente. El modelo así construido por Kazan et al. opera muy cerca del límite de Casimir para el scattering debido a la superficie, y por tanto con un MFP para los fonones próximo al diámetro de los NWs. Este modelo consigue un muy buen 
ajuste de los datos experimentales para los NWs lisos medidos en [11], pero para ello tiene que recurrir a un gran número de parámetros de ajuste, invalidando su utilización para predecir la conductividad térmica de NWs para cuyos diámetros no existen medidas experimentales.

Todos los modelos recopilados hasta este momento tratan el scattering de superficie cerca del límite difusivo, lo que impone un MFP para los fonones del orden de la longitud de Casimir, determinada en geometría cilíndrica por el diámetro del NW [28]. Esto implica que uno no puede reducir mucho la conductividad térmica actuando sobre el término de scattering de superficie utilizado en estos modelos. Por tanto ninguno de ellos puede ofrecer un buen ajuste a los datos experimentales para la conductividad térmica en NWs semiconductores de superficie rugosa como los medidos en [2].

Profundizando en el efecto que la morfología de la superficie introduce en el transporte térmico, F. Alvarez et al. desarrollaron un modelo en el que transporte fonónico se modela a través de una equivalencia con un problema de transporte hidrodinámico para un fluido [45]. Para ello resuelve la ecuación de Guyer-Krumhansl para el fluido fonónico en el interior de NW [46,47], obteniendo de este modo una sencilla expresión para la conductividad térmica, dependiendo ésta de la conductividad macroscópica, las dimensiones del NW y de la fracción de fonones que sufren un scattering especular en la superficie del NW [48]. Posteriormente, este mismo grupo, trabajando en la base de este modelo hidrodinámico, mostró como la rugosidad de la superficie podía causar una inversión local del flujo fonónico, lo que supone una limitación más agresiva que el límite difusivo, y causa que el MFP de los fonones pueda ser menor que determinado por el límite de Casimir [49]. Este resultado es consistente con un trabajo previo desarrollado por E. Moore et al. [50], quienes por otro camino también mostraron que la rugosidad superficial causaba eventos de backscattering que limitaban el transporte térmico al introducir una componte en sentido contrario al flujo fonónico que transporta la energía. Utilizando el modelo hidrodinámico de [45] y bajo esta hipótesis, A. Sellito et al. [51] encontraron un buen resultado para los valores de la conductividad térmica para NWs lisos de silicio por debajo de $150 \mathrm{~K}$ y que además ajustaba razonablemente bien a los datos experimentales para NWs de silicio rugosos de la referencia [2] en el mismo rango de temperatura. 


\section{TRANSPORTE TÉRMICO EN NWS SEMICONDUCTORES}

Tomando otro camino, P. Martin et al. estudiaron el efecto de la rugosidad en transporte térmico mediante teoría de perturbaciones [52], añadiendo además el efecto de una fina capa de $\mathrm{SiO}_{2}$ en superficie. Con este complejo modelo obtuvieron unos resultados próximos a los valores experimentales, sin alcanzar un buen ajuste, tanto para NWs de silicio lisos como rugosos. Por otro lado, en este trabajo se muestra como el efecto de la superficie es más importante cuanto más pequeño es el diámetro de los NWs; i.e. el efecto de la superficie es más relevante cuanto menor es el diámetro del NW.

Otros autores han recurrido a complejos modelos de simulación de dinámica molecular y atomística $[53,54,55,56]$, encontrando que la rugosidad superficial fomenta la aparición de modos fonónicos no propagativos que dificultan el transporte térmico. En uno de estos trabajos, M. Luisier encontró que no es necesario un valor muy elevado de $R M S$ para las superficies de los NWs para que los eventos de scattering en superficie fuesen difusivos [56]. Esto implica que incluso para NWs considerados lisos, como los medidos en [11], el MFP de los fonones es muy cercano al impuesto por límite de Casimir, y por tanto justifica que los modelos que trabajan en ese límite obtengan un buen resultado para los NWs lisos de silicio medidos en [11]. Un factor clavé en el papel jugado por la superficie en la resistencia al transporte térmico es el desempeñado por la longitud de correlación que caracteriza la rugosidad superficial. Este factor no se ha tenido en cuenta por ninguno de los modelos enumerados hasta el momento, dado que todos relacionan el scattering de superficie únicamente con el valor $R M S$ de la rugosidad superficial. Sin embargo se sabe experimentalmente que el efecto de superficie se debe a una combinación de ambos parámetros [22], por tanto un modelo capaz de predecir la conductividad térmica de NWs semiconductores rugosos tiene que incorporar ambas factores en los eventos de scattering de superficie.

J. Shadu et al. desarrollaron un modelo en el que ambos factores característicos de la rugosidad superficial [57], $R M S$ y longitud de correlación, se tienen en cuenta. En este trabajo se muestra como para longitudes de correlación de magnitud similar al valor RMS, un mayor número de fonones sufren eventos de scattering difusivo en la superficie, mientras que para valores de longitud de correlación grandes, únicamente los fonones de longitudes de onda grande sufren scattering difusivo. Dado 
que estos fonones son menos energéticos el transporte térmico se ve menos penalizado, y por tanto la conductividad térmica es mayor. Además mostraron como los fonones más energéticos son fuertemente atenuados en superficies con valores de RMS grandes $(R M S \sim 5 \mathrm{~nm})$ y longitudes de correlación cortas, resultando en valores de sus MFP por debajo de la longitud de Casimir, y por tanto en una atenuación más agresiva que la impuesta por el límite difusivo clásico. Utilizando este modelo los autores consiguen un ajuste muy bueno a los datos experimentales de NWs de silicio lisos y rugosos. Desafortunadamente, la obtención de una buena predicción de la conductividad térmica en NWs de silicio rugosos se consigue recurriendo a longitudes de correlación del orden de 50 a 100 nm, mientras que tal y como posteriormente J. Lim et al. mostraron experimentalmente, los valores para la longitud de correlación para NWs de silicio rugosos con conductividades térmicas equivalentes a los de la referencia [2] se encuentran en el rango de los 5-20 nm.

Por último, cuando se consideran NWs semiconductores hechos de aleaciones, uno debe considerar la perturbación que dicha aleación introduce en el transporte térmico. Esto resulta en una complicación adicional para obtener un buen modelo para la conductividad térmica. N. Yang et al. en una primera aproximación a este problema, mostraron que en NWs compuestos por distintos isotopos de silicio aparecía una gran reducción en la conductividad térmica [58], debida exclusivamente al scattering fonónico intrínseco a la aleación, con un mínimo absoluto para una aleación compuesta por el $50 \%$ de cada isotopo. Posteriormente, J. Chen et al. [58], realizaron una simulación de dinámica molecular para NWs de silicio-germanio obteniendo un resultado similar al de Yang et al. [58], encontrando además, que incluso para pequeñas proporciones de germanio en la aleación la conductividad térmica en los NWs se veía severamente reducida frente al valor de la conductividad térmica del mismo NW de silicio puro, tal y como posteriormente se demostró experimentalmente en [23]. Usando este modelo estimaron una reducción en la conductividad térmica del $50 \%$ para un NW con solo un $5 \%$ de germanio frente al valor del silicio puro volúmico, valor muy alejado del observado posteriormente experimentalmente, por lo que este modelo no puede utilizarse para predecir la conductividad térmica de NWs aleados. Por otro lado, Z. Wang et al. [60] trabajando en una modificación al modelo presentado por N. Mingo para NWs de silicio [30], desarrolló un 


\section{TRANSPORTE TÉRMICO EN NWS SEMICONDUCTORES}

modelo en el que todos los eventos de scattering fonónicos se toman como independientes para las componentes de silicio y de germanio, salvo el scattering debido a impurezas, que se toma como scattering de aleación y que depende de la composición a través de una función de mezcla cuadrática con la proporción de germanio en el NW; mediante esta hipótesis se obtienen unos resultados para la conductividad térmica en NWs de $S i_{1-x} G e_{x}$ del orden de los obtenidos experimentalmente en la referencia [23], sin ser por ello muy precisos.

A tenor de la cantidad de propuestas diferentes existentes para modelar la conductividad térmica de NWs semiconductores, existe un enorme interés por encontrar un modelo teórico para calcular la conductividad térmica en NWs semiconductores. En concreto, para el desarrollo de este trabajo de investigación es fundamental poder calcular de forma precisa la conductividad térmica de NWs de silicio y $S i_{1-X} G e_{X}$, tanto lisos como rugosos. Por desgracia ninguno de los modelos expuestos hasta el momento nos permite esto de forma precisa, y por ello se ha tenido que recurrir al desarrollo de un modelo propio que responda a las necesidades requeridas en otras partes de la investigación.

\subsection{Desarrollo de un modelo predictivo para la con- ductividad térmica en NWs de silicio y $\mathrm{Si}_{-} \mathbf{1}-\mathbf{X G e} \mathrm{X}_{\mathbf{x}}$}

\section{Consideraciones previas}

Uno de los pilares fundamentales sobre los que se sustenta la investigación recogida en esta tesis es como afectan los efectos térmicos a los espectros Raman obtenidos de NWs de silicio y silicio-germanio. Para entender esto, es necesario conocer bien al actor fundamental en los fenómenos de transporte térmico, que no es otro que la conductividad térmica de estas estructuras. Dado que esta magnitud es fuertemente dependiente de propiedades intrínsecas a cada NW -Diámetro y rugosidad superficial- y que solo está determinada experimentalmente para unos pocos NWs en unos rangos de temperatura limitados, va a resultar fundamental utilizar un modelo capaz de predecir la conductividad térmica en todos aquellos casos para los que no hay datos experimentales; es decir, necesitaremos un modelo que 
al menos prediga la conductividad térmica de NWs de silicio con cualquier diámetro comprendido entre los rangos para los que disponemos de NWs (30 nm a 120 $\mathrm{nm}$ aproximadamente) y para temperaturas mucho más elevadas que los 350-400 $\mathrm{K}$ para los que hay datos experimentales.

Como hemos podido observar en la sección anterior, existen multitud de propuestas diferentes para calcular la conductividad térmica en NWs semiconductores. Muchas de ellas funcionan bien a la hora de predecir comportamientos, pero tienen escasa precisión, otras son capaces de ajustar bien los resultados experimentales, pero únicamente para NWs considerados como lisos, mientras que otras ofrecen un buen ajuste para ambos tipos de NWs bajo hipótesis para el efecto de superficie no muy reales cuando se las compara con lo experimentalmente observado.

Debido a estas limitaciones, se ha optado por construir un modelo propio para la conductividad térmica que responda a las necesidades anteriormente expuestas. Además de la necesidad de contar con dicho modelo para el correcto desarrollo de la investigación aquí presentada, la obtención de un modelo para la conductividad térmica de NWs semiconductores capaz de predecir esta magnitud únicamente en función de las propiedades intrínsecas de cada NW tiene una gran relevancia por sí misma, ya que permite estimar el comportamiento térmico o termoeléctrico de nanodispositivos basados en estas estructuras sin necesidad de medir experimentalmente la conductividad térmica de cada NW utilizado.

\subsubsection{El formalismo de Callaway-Holland para la conductividad térmica en semiconductores}

El modelo de Callaway-Holland para el calculo de la conductividad térmica en semiconductores volúmicos ha sido ampliamente validado desde su concepción hace más de cincuenta años $[39,42,61]$. Además, este formalismo ha sido adaptado con relativo éxito para calcular la conductividad térmica en nanoescala $[17,26,34,40]$. A tenor de estos resultados, se escogió este formalismo como base para desarrollar un modelo capaz de ofrecer una precisa predicción de la conductividad térmica en NWs de silicio y silicio-germanio.

El formalismo de Callaway para el cálculo de la conductividad térmica se desarrolla sobre la base de la aproximación acústica, y por tanto su validez formal se reduce 


\section{TRANSPORTE TÉRMICO EN NWS SEMICONDUCTORES}

a temperaturas suficientemente bajas como para garantizar que esta aproximación es válida $[41,43]$. Sin embargo a través de sucesivas modificaciones al formalismo original, este modelo ha sido extendido con éxito en todo el rango de temperaturas para cristales semiconductores como el silicio o el germanio [39, 42]. Al sacar de rango de validez el formalismo original, los modelos basados en este formalismo deben ser tomados únicamente como herramientas de cálculo capaces de ofrecer una precisa predicción de la conductividad térmica .

El desarrollo del formalismo comienza con la ecuación de Boltzmann para la distribución de fonones en un semiconductor, dada por:

$$
\left(\frac{\partial N}{\partial t}\right)_{\mathbf{c}}-\mathbf{c} \nabla T \frac{d N}{d t}=0
$$

Donde $N$ es la densidad de fonones y c la velocidad de grupo de los mismos. En presencia de pequeños gradientes de temperatura $-\nabla T \sim 0$, la desviación del equilibrio de cada modo fononico $\mathbf{k}$ decae exponencialmente, y por tanto el decaimiento en la población de portadores puede describirse a través de un tiempo de relajación:

$$
\left(\frac{\partial N}{\partial t}\right)_{\mathbf{c}}=\frac{N^{0}-N}{\tau(\mathbf{k})}
$$

Aquí $N^{0}$ es la distribución de Planck, la cual describe la población fonónica en equilibrio y $\tau(\mathbf{k})$ representa el tiempo de relajación de cada modo. En este punto, Callaway separó el término izquierdo de la ecuación para tener en cuenta las contribuciones al tiempo de relajación de las componentes atribuidas a los eventos de scattering resistivos y scattering normal que afectan a los fonones [41]. Qué significa y a qué se deben estos procesos de scattering es el tema de la siguiente subsección, pudiéndose consultar en ella su naturaleza. Utilizando esta separación se obtiene:

$$
\left(\frac{\partial N}{\partial t}\right)_{\mathbf{c}}=\frac{N(\boldsymbol{\lambda})-N}{\tau_{N}}+\frac{N^{0}-N}{\left(\tau_{R}\right)}
$$

Donde $N(\boldsymbol{\lambda})$ es la función de Planck desplazada debida a la introducción de los procesos normales dada en primera aproximación como [62]:

$$
N(\boldsymbol{\lambda})=N(0)+\boldsymbol{\lambda}\left(\frac{\partial N}{\partial \boldsymbol{\lambda}}\right)_{\mathbf{0}}=N^{0}+\frac{\boldsymbol{\lambda} \boldsymbol{k}}{K_{B} T} \frac{e^{\hbar \omega / K_{B} T}}{\left(e^{\hbar \omega / K_{B} T}-1\right)^{2}}
$$


Por otro lado, dado que se trabaja en una aproximación en la que los gradientes de temperatura son pequeños, puede asumirse que $\frac{d N}{d t}=\frac{d N^{0}}{d t}$, y por tanto, la ecuación 2.2 puede simplificarse:

$$
\left(-\frac{\hbar \omega}{K_{B} T} \mathbf{c} \nabla T+\frac{1}{\tau_{N}} \frac{e^{\hbar \omega / K_{B} T}}{\left(e^{\hbar \omega / K_{B} T}-1\right)^{2}} \frac{\lambda \boldsymbol{k}}{K_{B} T}\right)-\left(\frac{1}{\tau_{N}}+\frac{1}{\tau_{R}}\right)\left(N-N^{0}\right)=0
$$

El término $\left(N-N^{0}\right)$ de la ecuación 2.6 puede reescribirse asumiendo que todo el proceso de decaimiento se rige por un tiempo de relajación global $\alpha(\mathbf{k})$, expresado como:

$$
\left(N-N^{0}\right)=\alpha(\mathbf{k}) \mathbf{c} \nabla T \frac{\hbar \omega}{K_{B} T^{2}}
$$

Al realizar esto, la ecuación de Boltzmann para el transporte fonónico dada en 2.2 se transforma en una ecuación más manejable:

$$
\left(\frac{\alpha(\mathbf{k})}{\tau_{C}}-1\right)\left(\frac{\hbar \omega}{T}\right) \mathbf{c} \nabla T+\frac{1}{\tau_{N}} \lambda k=0
$$

Donde $\tau_{C}=\left(\tau_{N}^{-1}+\tau_{R}^{-1}\right)^{-1}$.

Asumiendo que el semiconductor al que se quiere aplicar este formalismo puede considerarse un medio isotopo, $\boldsymbol{\lambda}$ debe ser un vector constante en la dirección del gradiente térmico. Bajo esta hipótesis, Callaway define un parámetro $\beta$ dado como $\beta=-\frac{\lambda}{\hbar v^{2}} \frac{T}{\nabla T}[41,43]$, siendo $v$ la velocidad del sonido del semiconductor. Trabajando en la aproximación acústica $\mathbf{k}=\frac{\mathbf{c} \omega}{v^{2}}$ [43], que introducido en 2.8 junto con $\beta$ permiten obtener una expresión para el tiempo de relajación absoluto $\alpha$ :

$$
\alpha=\tau_{C}\left(1+\left(\frac{\beta}{\tau_{N}}\right)\right)
$$

Utilizando este termino de relajación global para el proceso de decaimiento fonónico, la conductividad térmica puede estimarse a través del calor especifico fonónico:

$$
\kappa=\frac{1}{8 \pi^{3}} \sum_{i} \int_{0}^{2 \pi} \int_{0}^{\pi}\left(\int v_{i}^{2} \alpha_{i}(\mathbf{k}) C_{p h}^{i}(\mathbf{k}) \cos ^{2} \theta d^{3} \mathbf{k}\right) d \theta d \phi
$$

Donde $\theta$ es el ángulo formado entre el vector de onda de los fonones, $\mathbf{k}$, y el gradiente de temperatura, $\nabla T . C_{p h}$ es precisamente el calor especifico fonónico, que 


\section{TRANSPORTE TÉRMICO EN NWS SEMICONDUCTORES}

se obtiene como

$$
C_{p h}=\frac{d}{d T}\left(\hbar \omega N^{0}\right)=\frac{(\hbar \omega)^{2}}{K_{B} T^{2}} \frac{e^{\hbar \omega / K_{B} T}}{\left(e^{\hbar \omega / K_{B} T}-1\right)^{2}}
$$

Este desarrollo asume que solo los fonones acústicos contribuyen al transporte térmico. Callaway en su trabajo original no distinguió entre los fonones pertenecientes a las ramas longitudinal acústica y las dos transversales acústica, asumiendo simplemente que las tres contribuyen de igual modo al transporte. Sin embargo Holland mostró como esta simplificación no puede considerarse admisible, siendo necesario considerar la contribución especifica de cada una de las tres ramas [42]. De este modo la conductividad térmica se obtiene como:

$$
\kappa=\sum_{i} \frac{v_{i}^{2}}{8 \pi^{3}} \int_{0}^{2 \pi} \int_{0}^{\pi}\left(\int\left(\frac{(\hbar \omega)^{2}}{K_{B} T^{2}}\right) \frac{1}{\tau_{C}}\left(1+\left(\frac{\beta}{\tau_{N}^{i}}\right)\right) \frac{e^{\hbar \omega / K_{B} T}}{\left(e^{\hbar \omega / K_{B} T}-1\right)^{2}} \cos ^{2} \theta d^{3} \mathbf{k}\right) d \theta d \phi
$$

Donde el subindice $i$ se refiere a cada una de las diferentes ramas acústicas, $i=$ $\{L A, T A 1, T A 2\}$.

Para evaluar la ecuación 2.12 y obtener la conductividad térmica del semiconductor cristalino aún es necesario determinar la forma explicita del parámetro $\beta$. Ésta se puede obtener de las reglas de conservación para el momento de los fonones en los eventos de scattering normal:

$$
\int\left(\frac{\partial N_{\mathbf{k}}}{\partial t}\right)_{N} \mathbf{k} d k=\int \operatorname{frac} N(\boldsymbol{\lambda})-N \tau_{N}^{i} \mathbf{k} d k
$$

Si se introduce en 2.13 el resultado de 2.7, se obtiene la ecuación:

$$
\int \frac{e^{\hbar \omega / K_{B} T}}{\left(e^{\hbar \omega / K_{B} T}-1\right)^{2}}\left(\frac{\hbar \omega}{K_{B} T^{2}}\right) \mathbf{c} \nabla T(\alpha(\mathbf{k})-\beta) \frac{\mathbf{c} \omega}{\tau_{N}^{i} v_{i}^{2}} d^{3} \mathbf{k}=0
$$

Donde de nuevo podemos introducir el resultado derivado en 2.9 para $\alpha$, y utilizar que $\|\mathbf{c} \nabla T\|=v_{i}^{2} \cos ^{2} \theta$ para obtener:

$$
\int \frac{e^{\hbar \omega / K_{B} T}}{\left(e^{\hbar \omega / K_{B} T}-1\right)^{2}}\left(\frac{\hbar \omega}{K_{B} T^{2}}\right)\left(\tau_{C}^{i}+\left(1-\frac{\tau_{C}^{i}}{\tau_{N}^{i}}\right) \beta\right) \frac{\omega}{\tau_{N}^{i} v_{i}^{2}} v_{i}^{2} \cos ^{2} \theta d^{3} \mathbf{k}=0
$$


De donde, dada la independencia ente $\beta$ y $k$, se obtiene que [43]:

$$
\beta=\frac{\int \frac{\tau_{C}^{i}}{\tau_{N}^{i}} \frac{e^{\hbar \omega / K_{B} T}}{\left(e^{\hbar \omega / K_{B} T}-1\right)^{2}}\left(\frac{\hbar \omega}{K_{B} T^{2}}\right) \omega \cos ^{2} \theta d^{3} \mathbf{k}}{\int\left(1-\frac{\tau_{C}^{i}}{\tau_{N}^{i}}\right)\left(\frac{1}{\tau_{N}^{i}}\right) \frac{e^{\hbar \omega / K_{B} T}}{\left(e^{\hbar \omega / K_{B} T}-1\right)^{2}}\left(\frac{\hbar \omega}{K_{B} T^{2}}\right) \omega \cos ^{2} \theta d^{3} \mathbf{k}}
$$

Este resultado es introducido en 2.12, y sabiendo además que $d^{3} k=\frac{\omega^{2}}{v^{3}} d \omega d \Omega_{k}=$ $\frac{\omega^{2}}{v^{3}} \sin \theta d \theta d \phi d \omega$, junto con el cambio de variable $x=\frac{\hbar \omega}{K_{B} T}$, nos permite expresar la conductividad térmica del semiconductor como:

$$
\begin{aligned}
& \kappa=\sum_{i}\left(\frac{K_{B}^{4} T^{3}}{8 \pi^{3} \hbar^{3} v_{i}}\right)\left(\int_{0}^{\pi} \int_{0}^{2 \pi} \int_{0}^{\frac{\theta_{i}}{T}}\left[\tau_{C}^{i} \frac{x^{4} e^{x}}{\left(e^{x}-1\right)^{2}} \cos ^{2} \theta \sin \theta d \theta d \phi d x\right]+\right. \\
& +\left[\frac{\int_{0}^{\pi} \int_{0}^{2 \pi} \int_{0}^{\frac{\theta_{i}}{T}}\left[\frac{\tau_{C}^{i}}{\tau_{N}^{i}} \frac{x^{4} e^{x}}{\left(e^{x}-1\right)^{2}} \cos ^{2} \theta \sin \theta d \theta d \phi d x\right]}{\int_{0}^{\pi} \int_{0}^{2 \pi} \int_{0}^{\frac{\theta_{i}}{T}}\left[\left(1-\frac{\tau_{C}^{i}}{\tau_{N}^{i}}\right)\left(\frac{1}{\tau_{N}^{i}}\right) \frac{x^{4} e^{x}}{\left(e^{x}-1\right)^{2}} \cos ^{2} \theta \sin \theta d \theta d \phi d x\right]}\right] \times \\
& \left.\times \int_{0}^{\pi} \int_{0}^{2 \pi} \int_{0}^{\frac{\theta_{i}}{T}}\left[\frac{\tau_{C}^{i}}{\tau_{N}^{i}} \frac{x^{4} e^{x}}{\left(e^{x}-1\right)^{2}} \cos ^{2} \theta \sin \theta d \theta d \phi d x\right]\right)
\end{aligned}
$$

Donde se define $\theta_{i}$ como la temperatura de corte característica para cada rama fonónica, inicialmente vinculada con la temperatura de Debye del material [41, 43].

\subsubsection{Mecanismos de scattering fonónico}

La aplicación del formalismo de Callaway para obtener la conductividad térmica de un determinado material en un rango de temperaturas desde $1 \mathrm{~K}$ a más de 1000 K con precisión recae en la apropiada caracterización de los diferentes mecanismos de scattering fonónico, que son los responsables de la limitación al transporte energético por parte de los fonones de la red.

\section{Scattering por procesos que implican tres fonones}

Este tipo de eventos está determinado únicamente por la interacción entre fonones y sucede de dos formas diferentes. La primera forma implica que un fonón caracterizado por un vector de onda $q_{1}$ se combina con un segundo fonón $q_{2}$, resultado de esta interacción un nuevo fonón $q_{3}$. La segunda forma implica el decaimiento de 


\section{TRANSPORTE TÉRMICO EN NWS SEMICONDUCTORES}

un fonón $q_{1}$ en otros dos fonones $q_{2}$ y $q_{3}$ respectivamente.

$$
\begin{aligned}
& q_{1}+q_{2}=q_{3} \\
& q_{1}=q_{2}+q_{3}
\end{aligned}
$$

En ambos procesos debe conservarse la energía total; sin embargo, esta conservación no tiene por que repetirse para el momento. Aquellos procesos a tres fonones que no conservan el momento se denominan eventos de scattering umklapp, mientras que los que si lo conservan se denominan eventos de scattering normal.

En los procesos umklapp el momento inicial y final difiere en un vector de la red reciproca no nulo, por lo que los fonones resultantes caen fuera de la primera zona de Brillouin; por tanto, las reglas de selección para estos eventos pueden expresarse como:

$$
\begin{array}{r}
q_{1}+q_{2}=q_{3}=q_{3}^{\prime}+G \\
q_{1}=q_{2}+q_{3}=q_{2}+q_{3}^{\prime}+G
\end{array}
$$

Donde $q_{3}^{\prime}$ es el fonón "reflejado" en la primera zona de Brillouin y G un vector de la red reciproca [28]. Este fonón $q_{3}^{\prime}$ se caracteriza por un vector de onda opuesto a $q_{1}$ y $q_{2}$, resultando en un proceso resistivo al flujo térmico.

Estos eventos fueron estudiados por R.E. Peierls, quien propuso una forma para el tiempo de relajación característico dada por $\tau_{u}^{-1} \propto T^{a} e^{\theta / b T}[62]$, donde $\theta_{i}$ es aquí la temperatura de corte característica y $a$ y $b$ dos parámetros a determinar $[62,63,64,65]$. Posteriormente, G.A Slack mostró que en un amplio rango de temperaturas el resultado de R.E. Peierls podía expresarse como $\tau_{u}^{-1}=$ $B \omega^{2} T e^{\theta / 3 T}$ [64]. Utilizando esta forma en el modelo de Callaway-Holland, demostró que podían modelarse las conductividades térmicas de distintos cristales puros con un buen acuerdo a los datos experimentales [64].

Por otro lado, P.G. Klemens estudiando estos procesos en alta temperatura concluyó que su contribución a los procesos resistivos estaba caracterizada por un tiempo de relajación dado por $\tau_{u}^{-1}=C \omega^{2} T$ [65]. La existencia de estas dos formas describiendo el mismo proceso no resulta en ninguna discrepancia, ya que para alta temperatura, la parte exponencial de la forma propuesta por G.A. Slack 
tiende a uno, y por tanto ambas expresiones deben coincidir. El parámetro $C$ fue evaluado exactamente por P.G. Klemens, obteniendo que $C=\frac{2 \gamma^{2} \hbar}{M v^{2} \theta}$, donde $\gamma$ es el parámetro de Grüneisen del material. Por tanto, teniendo en cuenta la separación en las diferentes ramas fonónicas propuesta por Holland, los procesos umklapp en un amplio rango de temperatura se pueden describir como:

$$
\left(\tau_{U}^{i}\right)^{-1}=\frac{2 \gamma_{i}^{2} \hbar}{M v_{i}^{2} \theta_{i}} \omega^{2} T e^{\theta_{i} / 3 T}
$$

Volviendo sobre los procesos a tres fonones conservativos, su contribución a la resistencia térmica ha sido ignorada por muchos autores [25, 27, 30, 31,33]. Sin embargo, su contribución si que afecta de forma indirecta a la conductividad térmica redistribuyendo la población de fonones. Este mecanismo fue explicado en su momento por C. Herring, quien evaluó como afecta la distribución de momento fonónico entre las diferentes bandas acústicas [32] al transporte térmico; encontró que la relajación de un fonón transversal acústico, consistente en su interacción con un fonón longitudinal acústico, derivaba en un otro fonón longitudinal. Por otro lado, la relajación de los fonones longitudinales ocurría de dos formas, su decaimiento en dos fonones transversales o bien por la creación de un fonón longitudinal de dos transversales. Esta redistribución entre bandas contribuye al scattering total a través de un tiempo de relajación descrito por Herring como [32]:

$$
\begin{gathered}
\left(\tau_{N}^{L}\right)=C_{L} \omega^{2} T^{3} \simeq \frac{K_{B}^{3} \gamma_{L}^{2} V}{M \hbar^{2} v_{L}^{5}} \omega^{2} T^{3} \\
\left(\tau_{N}^{T}\right)=C_{T} \omega T^{4} \simeq \frac{K_{B}^{4} \gamma_{L}^{2} V}{M \hbar^{3} v_{L}^{5}} \omega T^{4}
\end{gathered}
$$

Donde las expresiones de los coeficientes $C_{i}$ se han aproximado por los valores obtenidos por M. Asen-Palmer et al. en [66] siendo $V$ el volumen de la celda unidad.

\section{Scattering debido a la presencia de diferentes isotopos o impurezas en el cristal}

En un cristal real, las impurezas dificultan el transporte del flujo fonónico, constituyendo centros de scattering para los fonones. Incluso en cristales puros, sin 


\section{TRANSPORTE TÉRMICO EN NWS SEMICONDUCTORES}

presencia de otros elementos, existen diferentes isotopos del mismo elemento conviviendo juntos. Estos isotopos constituyen un elemento resistivo muy importante para fonones de alta frecuencia, por lo que su efecto debe ser tenido en cuenta. La perturbación en la red cristalina introducida por estos átomos substitucionales ha sido evaluada por P.G. Klemens utilizando teoría de perturbaciones [44, 65], obteniendo una expresión para el tiempo de relajación característico debido a esta mezcla isotópica dada como:

$$
\left(\tau_{I}^{i}\right)^{-1}=\frac{V \Gamma}{2 \pi v_{i}^{3}} \omega^{4}
$$

Donde $\Gamma$ es parámetro de fluctuación de masa, definido para un único elemento como $\Gamma=\sum_{j} f_{j}\left(1-\frac{M_{j}}{M}\right)^{2}$, siendo $f_{j}$ la fracción de abundancia atómica del isotopo $j$ en el cristal.

\section{Eventos de scattering debidos a la superficie}

Un cristal real, a diferencia de uno ideal, tiene un tamaño finito, y esa finitud introduce una importante restricción al transporte fonónico actuando como regiones muy eficientes a la hora de producir eventos de scattering. Este limitación de escala es especialmente relevante en nanoescala, donde las superficies actúan confinando el recorrido libre medio (MFP) de los fonones al tamaño de la nanoestructura, constituyéndose en el principal responsable de la baja conductividad térmica de estas estructuras.

El problema del scattering debido a la superficie ha sido tratado profundamente por J.M. Ziman sobre la base de la teoría de Casimir para el scattering de portadores [28]. El proceso puede explicarse bajo una visión fenomenológica considerando dos tipos diferentes de eventos para un fonón que llega a la frontera del cristal; el primero, denominado evento especular, implica que el fonón al llegar a la superficie se "refleja" al interior del cristal contribuyendo de nuevo al transporte energético en la dirección del gradiente térmico. El segundo, denominado evento difusivo, implica que el fonón que llega a la superficie deja de contar para el transporte energético. El primer tipo de eventos es el que ocurre en superficies perfectamente lisas, mientras que el segundo ocurrirá en superficies completamente rugosas. En el limite intermedio entre estos regímenes, los fonones que se encuentran con la 
superficie tendrán una probabilidad $p$ de sufrir un evento especular, y por tanto una probabilidad $1-p$ de sufrir un scattering difusivo. Bajo esta hipótesis, J.M Ziman derivó una expresión para cuantificar el MFP de los fonones en una muestra finita dada por [28]:

$$
\Lambda=\frac{1+p}{1-p} \Lambda_{0}
$$

Siendo $\Lambda_{0}$ el MFP atribuido a una superficie donde todos los eventos son difusivos [28]. El valor de $\Lambda_{0}$ depende de la geometría de la muestra, correspondiendo en el caso de un cilindro a su diámetro, i.e. $\Lambda_{0}=D$; a este MFP se le conoce como limite de Casimir para el portador. La correcta cuantificación de la aportación introducida por las superficies a la resistencia térmica recae por tanto en la forma adoptada por la función de probabilidad $p$, que obviamente está relacionada con la morfología de la superficie. Comúnmente esta probabilidad ha sido tomada como cero $[25,30,33,37]$, o como una constante independiente de la temperatura y la frecuencia $[27,34,35]$; simplificando así la expresión para el tiempo de relajación debido a la superficie. Sin embargo, J.M. Ziman mostró que la probabilidad del evento especular depende de la longitud de onda de los fonones incidentes y de la forma de la superficie [28]. El efecto de la morfología de la superficie se introduce a través de un parámetro $\eta$, denominado parámetro de aspereza, que no es otro que el RMS de la superficie.

En primera aproximación la función de probabilidad puede expresarse como $p=$ $e^{-\frac{16 \pi^{3} \eta^{3}}{\lambda^{2}}}$, y ha sido utilizada por algunos autores para obtener la conductividad térmica en NWs semiconductores [50]. Sin embargo, y pese a ser una mejor descripción que asumir $p$ cero o constante, esta descripción para la probabilidad especular ha sido considerada por P.G. Ziman como poco fiel a la realidad, dado que asume una distribución homogénea de las rugosidades en superficie [28]. En vez de esto, el propio P.G. Ziman desarrollo una forma alternativa introduciendo una distribución de probabilidad para la rugosidad superficial; de este modo la probabilidad de un evento especular se define como:

$$
p(\eta, \lambda)=\int P(\eta) e^{-\frac{16 \pi^{3} \eta^{3}}{\lambda^{2}}} d \eta
$$




\section{TRANSPORTE TÉRMICO EN NWS SEMICONDUCTORES}

Esta expresión aún puede simplificarse más si uno tiene en cuenta que probabilidad de reflexión es una medida de la fracción de área cuyo valor de RMS es menor que $\lambda / 4 \pi[28]$, obteniéndose entonces que $p(\eta, \lambda) \approx \int_{0}^{\lambda / 4 \pi} P(\eta) d \eta$. Sobre esta forma, Mingo et al. construyeron una aproximación para el cálculo de la conductividad térmica de NWs de silicio rugosos en el limite de baja temperatura [67], utilizando para ello un función de distribución $P(\eta)=\frac{1}{\eta} e^{-\frac{\eta}{\eta_{0}}}$.

Por otro lado, basándose en la dependencia de la probabilidad del evento especular con la frecuencia y la morfología de la superficie, S.B. Soffer construyo otra forma para $p$ en presencia de un flujo de portadores que se desplaza en dirección de un gradiente [68]:

$$
p_{i}(\eta, \omega)=e^{-\left(\frac{2 \eta \sin \theta}{v_{i}}\right)^{2}}
$$

Donde $\eta$ vuelve a ser el valor RMS de la superficie y $\theta$ el ángulo formado entre el vector de onda de los fonones incidentes y el gradiente de temperatura.

Finalmente, la forma tomada para la tasa relajación debida a la superficie se construye a través de la forma para el MFP dada en 2.23, añadiendo la limitación en longitud, $L$, de la muestra y tomando para la probabilidad especular la forma dada por 2.25, i.e:

$$
\left(\tau_{B}^{i}\right)^{-1}=\left(\frac{1-e^{-\left(\frac{2 \eta \sin \theta}{v_{i}}\right)^{2}}}{1+e^{-\left(\frac{2 \eta \sin \theta}{v_{i}}\right)^{2}}} \Lambda_{0}^{-1}+L^{-1}\right) v_{i}
$$

Se ha escogido para la construcción del modelo la forma 2.25 para la probabilidad de evento especular ya que ha probado ser la que mejor resultado ofrece cuando se compara con los resultados experimentales.

\subsubsection{Cálculo de la conductividad térmica}

\subsubsection{Validación del modelo para semiconductores volúmicos}

El cálculo de la conductividad térmica se realiza mediante la ecuación 2.17 en la que se introducen los tiempos de relajación dados en 2.20, 2.21, 2.22 y 2.26. Los términos $2.20,2.22$ y 2.26 constituyen el tiempo de relajación resistivo, que se 
construye a través de la regla de Matthiessen como:

$$
\tau_{R}^{i}=\left(\left(\tau_{U}^{i}\right)^{-1}+\left(\tau_{I}^{i}\right)^{-1}+\left(\tau_{B}^{i}\right)^{-1}\right)^{-1}
$$

Con esto, y especificando los valores de los parámetros intrínsecos para cada material, puede evaluarse el valor de la conductividad térmica. El cálculo de la compleja ecuación integral dada por 2.17 puede abordarse de diferentes maneras. Callaway y Holland, asumiendo muchas simplificaciones, la transformaron en diferentes ecuaciones exactas dependiendo del rango de temperaturas donde se utilizaba [41, 42]. Sin embargo en este trabajo se busca una solución precisa y general, por lo que se va a resolver completamente la ecuación 2.17, recurriendo para tal fin al cálculo numérico. Para ello se ha recurrido al software de cálculo numérico Mathematica de WolframResearch, el cual incorpora diversos y potentes métodos de integración numérica. El cálculo de la conductividad térmica mediante 2.17 requiere trabajar ajustando distintos parámetros a los datos experimentales, dos en el caso de materiales volúmicos y hasta cinco en el caso de NWs rugosos. Este proceso se realiza por prueba y error hasta conseguir el ajuste más preciso a los datos experimentales, por lo que es necesario que la evaluación de 2.17 sea lo más rápida y precisa posible, dado que se deben repetir los cálculos miles de veces para obtener una buena determinación de los parámetros de ajuste.

La comprobación de la validez del modelo y la elección del método numérico se lleva a cabo calculando la conductividad térmica del silicio volúmico y del germanio volúmico, para lo que se obtienen los resultados mostrados en la figura 2.5, utilizando los valores de la tabla 2.1. Como puede observarse el ajuste a los datos experimentales, tanto para el silicio como para el germanio, es muy bueno en todo el rango de temperaturas, desde temperaturas cercanas al Kelvin a valores elevados de más de un millar de Kelvin. Por otro lado, el cálculo de la conductividad térmica para estos materiales volúmicos nos mostró que el método de integración numérica con mejor relación entre velocidad y precisión para resolver la ecuación 2.17 es el basado en la regla de integración de Gauss-Kronrod localmente adaptativa [69]. Este método resulta mucho más adecuado que otros basados en reglas de tipo Regla del trapecio, Clenshaw-Curtis o Newton-Cotes, que en nuestro equipo se mostraron varías veces más lentos a la hora de resolver el problema bajo los mismos requisitos 
de precisión.
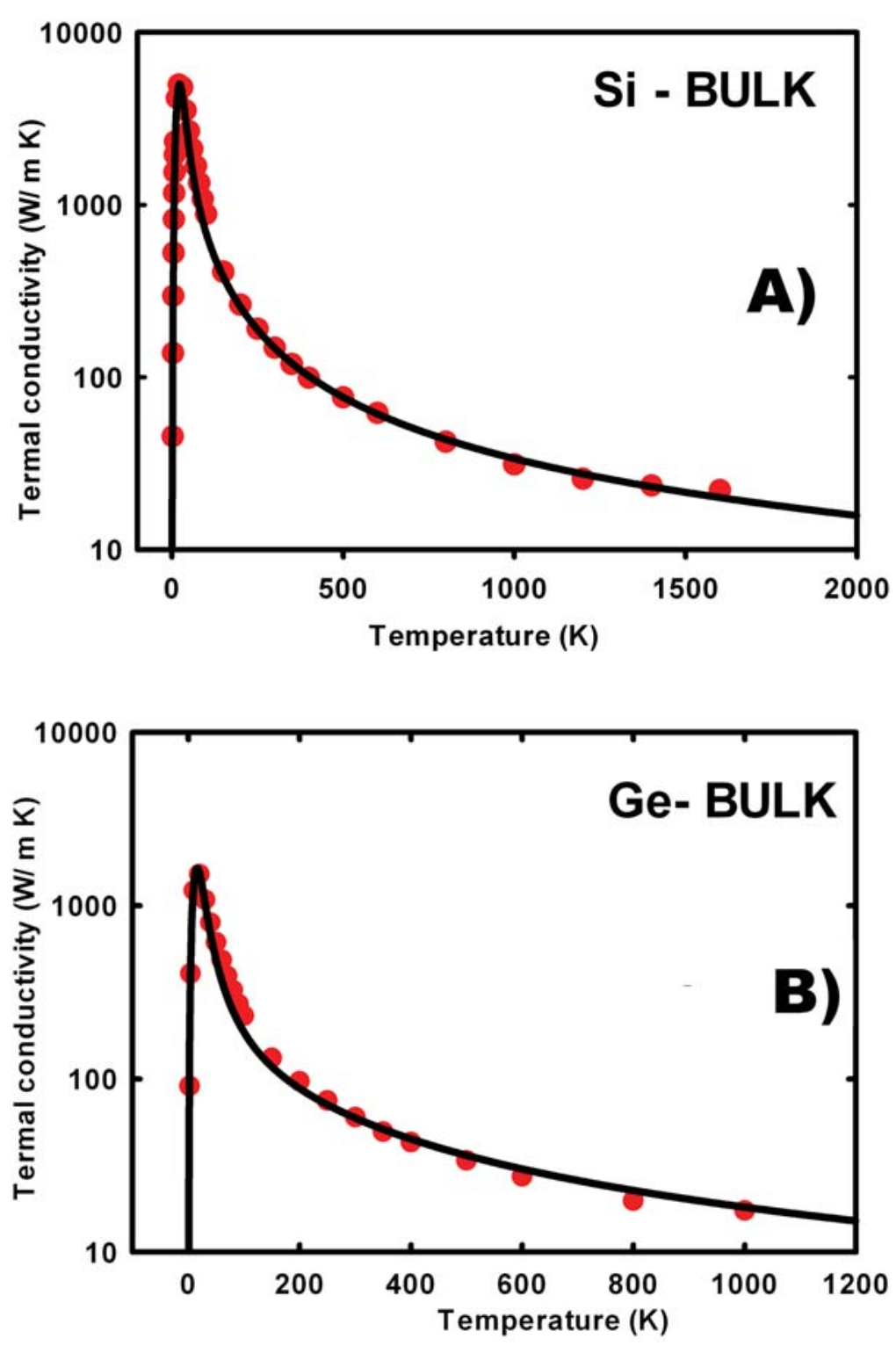

Figura 2.5: Conductividades térmicas experimentales para cristales volúmicos de silicio (A) y germanio (B) -puntos rojos- y conductividad térmica calculada con nuestro modelo de Callaway-Holland. Puede observarse el buen ajuste a los datos experimentales tomados de la referencia [14] en todo el rango de temperaturas 


\begin{tabular}{|c|c|c|c|c|c|c|c|c|c|c|}
\hline & $\mathrm{L}(\mathrm{m})$ & $\Lambda_{0}(m)$ & $\eta(m)$ & $\Gamma$ & $\theta_{L}(K)$ & $\theta_{T}(K)$ & $v_{L}(m / s)$ & $v_{T}(m / s)$ & $\gamma_{L}$ & $\gamma_{T}$ \\
\hline $\mathrm{Si}$ & $6 \times 10^{-3}$ & $6 \times 10^{-3}$ & $1 \times 10^{-9}$ & $2.1610^{-4}$ & 627.7 & 435.8 & 8430 & 5840 & 1.1 & 0.66 \\
\hline $\mathrm{Ge}$ & $4 \times 10^{-3}$ & $4 \times 10^{-3}$ & $1 \times 10^{-9}$ & $6.08 \times 10^{-4}$ & 387.1 & 278.3 & 4920 & 3540 & 1.1 & 0.66 \\
\hline
\end{tabular}

Cuadro 2.1: Parámetros utilizados en el cálculo de la conductividad térmica del silicio volúmico y germanio volúmico.

Los valores de los parámetros utilizados para el calculo de las conductividades térmicas mostrados en la figura 2.5, y resumidos en la tabla 2.1, requieren ciertas consideraciones. Los valores de $\Gamma$ y $\gamma_{i}$ están tomados de la referencia [61]; el parámetro de Grüneisen para cada rama, $\gamma_{i}$, se ha aproximado por un valor constante. Esta puede parecer una simplificación muy drástica, dado que es bien conocida la dependencia de este parámetro con la temperatura, especialmente a bajas temperaturas [44]. Sin embargo, en el marco de trabajo construido, esta simplificación es aplicable; el parámetro de Grüneisen interviene en los eventos de scattering a tres fonones, cuya expresión genérica puede escribirse como:

$$
\left(\tau_{j}^{i}\right)^{-1}=K_{j}^{i} \times f_{j}^{i}(\omega) \times g_{j}^{i}(T)
$$

Es decir una grupo $K_{j}^{i}$ de parámetros intrínsecos de cada material multiplicando a funciones de la frecuencia y la temperatura. En esta construcción, toda la dependencia de los eventos de scattering con $\omega$ y con $T$ está englobada en las funciones $f_{j}^{i}$ y $g_{j}^{i}$, mientras que el grupo $K_{j}^{i}$ es independiente de estas magnitudes. Por tanto toda dependencia con la frecuencia y la temperatura de los parámetros del grupo $K_{j}^{i}$, como por ejemplo el parámetro de Grüneisen, está englobada en $f_{j}^{i}$ y $g_{j}^{i}$, apareciendo en $K_{j}^{i}$ únicamente su parte constante.

Las velocidades del sonido para las ramas transversal y longitudinal de ambos materiales proceden de medidas experimentales y están tomadas de la referencia [44]. El valor de la RMS de la superficie,i.e. el valor de $\eta$, está tomado considerando una muestra formada por superficies lisas, y por tanto, se ha tomado un valor idéntico al reportado para superficies lisas en NWs [40]. Por otro lado, los valores que caracterizan las dimensiones de la muestra, $L$ y $\Lambda_{0}$, comúnmente toman idéntico valor para muestras macroscópicas [61], y su valor se sitúa en el rango de los milímetros. Con esté tamaño volúmico, el scattering debido a la superficie apenas afecta a la conductividad térmica a temperaturas por encima de $150 \mathrm{~K}$, siendo relevan- 


\section{TRANSPORTE TÉRMICO EN NWS SEMICONDUCTORES}

te únicamente a baja temperatura cuando el MFP de los fonones es muy grande. Considerando esto, el valor de $L$ y $\Lambda_{0}$ se fija para cada material de tal modo que la conductividad térmica calculada coincida con el máximo que la conductividad térmica alcanza a baja temperatura $[39,61]$.

Finalmente, la determinación de las temperaturas de corte, $\theta_{i}$, para los modos longitudinal y transversales se realiza por ajuste a los datos experimentales para $T>200 K$. En el modelo original de Callaway, estas temperaturas se hacen coincidir con la temperatura de Debye para el material; sin embargo, la evaluación de esta temperatura de Debye depende del método utilizado para su determinación y no ofrece un resultado único. Cuando uno obtiene la temperatura de Debye para el silicio volúmico a partir del calor especifico se obtiene un valor de $645 \mathrm{~K}$ [61]. Por otro lado, esta temperatura también puede obtenerse a partir de las velocidades del sonido de las ramas acústicas, lo que arroja un valor cercano a $710 \mathrm{~K}$ [61]. Por otro lado, si uno estima el valor de la temperatura de Debye a través de las frecuencias de limite de zona de los fonones acústicos, el valor obtenido es mucho más pequeño, siendo de tan solo $298 \mathrm{~K}$ [61]. Aparentemente el valor más exacto es el obtenido con el último método [39,40,61], pero tomando este límite solo se consideraría el transporte debido a los modos acústicos. Sin embargo, en la referencia [37] se demuestra que el transporte debido a los fonones ópticos no puede ser ignorado, pudiendo ser incorporado a nuestro modelo a través de un incremento de estas temperaturas de corte [44]. Precisamente para incorporar la contribución óptica al transporte térmico se dejan como parámetros de ajuste las temperaturas de corte. Mediante este ajuste a los datos experimentales, se han obtenido unas temperaturas de corte de $495 \mathrm{~K}$ para el silicio y $307 \mathrm{~K}$ para el germanio, valores calculados a partir de $\theta_{\text {corte }}=3\left(\theta_{L}^{-1}+\theta_{T}^{-1}\right)^{-1}$ [70]. Como puede observarse estos valores se encuentran comprendidos entre los valores calculados por los distintos métodos para las temperaturas de Debye, y por tanto totalmente compatibles con ellos, siendo algo superiores a las temperaturas más bajas que se refieren sólo a los fonones acústicos; esto se debe a que las calculadas por ajuste engloban la aportación de los fonones ópticos al transporte térmico [44]. 


\subsubsection{Aplicación del modelo a NWs de silicio}

El modelo construido para el cálculo de la conductividad térmica ha demostrado un excelente comportamiento en escala macroscópica, siendo capaz de ajustar de forma precisa en todo el rango de temperatura los valores de la conductividad térmica experimental para cristales de silicio y germanio. Para los NWs lisos de silicio medidos en la referencia [11] se ha seguido el mismo procedimiento descrito anteriormente para los cristales volúmicos, utilizando para ello los valores resumidos en la tabla 2.2, obteniéndose con ellos el resultado mostrado en la figura 2.6.

\begin{tabular}{|c|c|c|c|c|c|c|c|c|c|c|}
\hline & $\mathrm{L}(\mathrm{m})$ & $\Lambda_{0}(m)$ & $\eta(m)$ & $\Gamma$ & $\theta_{L}(K)$ & $\theta_{T}(K)$ & $v_{L}(m / s)$ & $v_{T}(\mathrm{~m} / \mathrm{s})$ & $\gamma_{L}$ & $\gamma_{T}$ \\
\hline 115 & $5 \times 10-6$ & $115 \times 10-9$ & $1 \times 10-9$ & $2.16 \times 10-4$ & 537.5 & 303 & 7962 & 4741 & 1.1 & 0.66 \\
\hline 56 & $5 \times 10-6$ & $56 \times 10-9$ & $1 \times 10-9$ & $2.16 \times 10-4$ & 511.5 & 281 & 7200 & 4180 & 1.1 & 0.66 \\
\hline 37 & $5 \times 10-6$ & $37 \times 10-9$ & $1 \times 10-9$ & $2.16 \times 10-4$ & 484.5 & 250.1 & 7060 & 4106 & 1.1 & 0.66 \\
\hline 22 & $5 \times 10-6$ & $22 \times 10-9$ & $1 \times 10-9$ & $2.16 \times 10-4$ & 458 & 113.5 & 7018 & 4030 & 1.1 & 0.66 \\
\hline
\end{tabular}

Cuadro 2.2: Parámetros utilizados en el cálculo de la conductividad térmica de los NWs lisos de silicio medidos en la referencia [11].

La correspondencia entre la conductividad térmica calculada mediante nuestro modelo y los datos experimentales para la conductividad térmica es excelente en todo el rango de temperaturas para los NWs por encima del limite de confinamiento fonónico, $D>25 \mathrm{~nm}$. Sin embargo, se pierde precisión por debajo de este límite, como puede observarse en el ajuste a los datos experimentales para el NW de 22 nm. Pese a esto, los valores calculados para este NW se acercan bastante a los experimentales, mostrando un mejor comportamiento que la mayoría de los modelos resumidos al inicio del capítulo. Los NWs por debajo del limite de confinamiento fonónico muestran un comportamiento lineal con la temperatura, siendo dicho comportamiento imposible de reproducir por un modelo basado en el formalismo de Callaway-Holland, lo que sugiere que para calcular la conductividad térmica de estos NWs delgados hay que recurrir a otro tipo de modelos como el descrito en [36].

Fijándonos en los valores de la tabla 2.2, uno puede observar que no todos los valores utilizados coinciden con los utilizados para el cálculo de la conductividad térmica de silicio volúmico resumidos en la tabla 2.1. Obviamente los parámetros relacionados con la escala son diferentes, siendo L la longitud del NW y $\Lambda_{0}$ igual al diámetro; conviene recordar que $\Lambda_{0}$ es el limite de Casimir y que para geometría 


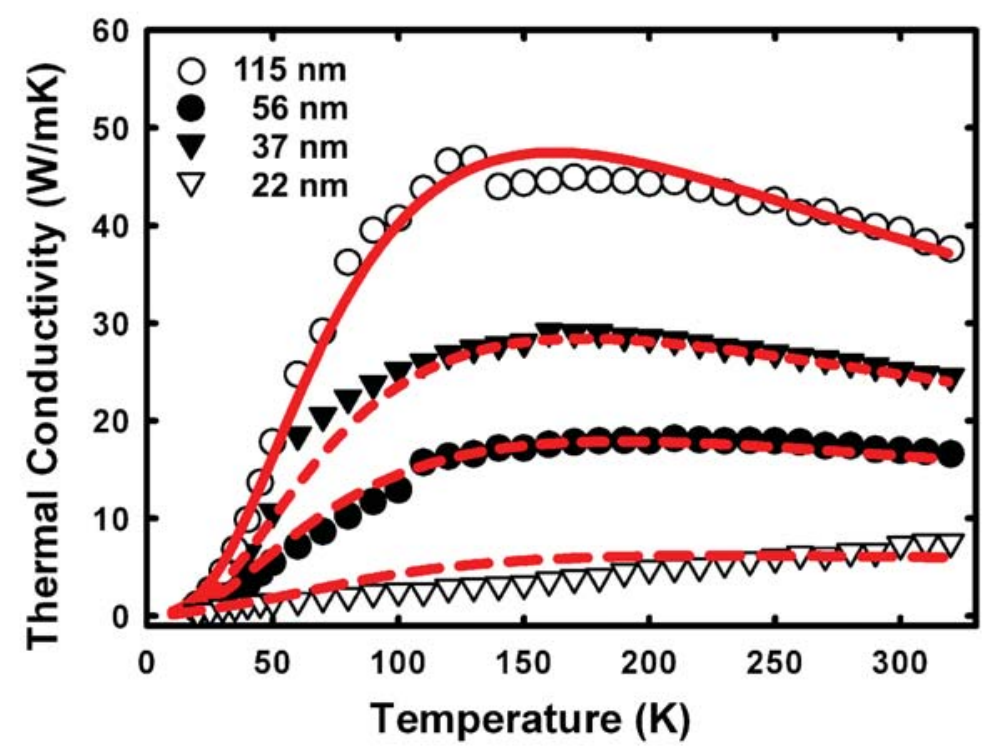

Figura 2.6: Conductividades térmicas experimentales de cuatro NWs lisos de silicio con diámetros de $115 \mathrm{~nm}$-círculos blancos-, $56 \mathrm{~nm}$-triángulos negros-, $37 \mathrm{~nm}$-círculos negros- y $22 \mathrm{~nm}$-triángulos blancos- obtenidos de la referencia 11. Las lineas muestran la conductividad térmica calcula mediante nuestro modelo para estos NWs, exhibiendo un excelente ajuste en todo el rango de temperatura para el existen datos experimentales 
cilíndrica coincide con el diámetro del NW. El parámetro de Grüneisen, $\gamma$ se toma igual que el del silicio volúmico, mientras que el parámetro que caracteriza la superficie, $\eta$, toma el valor dado para superficies lisas [40].

Finalmente quedan por analizar las velocidades del sonido y las temperaturas de corte de cada una de las ramas acústicas. Como ya se mencionó en la introducción de este capítulo, diversos autores han mostrado que las velocidades del sonido para los modos longitudinal y transversales en NWs son más bajas que las medidas en sus homólogos volúmicos, incluso para aquellos NWs con diámetros para los que el confinamiento fonónico apenas afecta $[25,27,34,40,70]$. Al igual que ocurría para las temperaturas de corte, estas velocidades pueden obtenerse por distintos métodos que no arrojan un resultado único, y por lo tanto su valor se obtendrá del ajuste a los datos experimentales. Por otro lado, las temperaturas de corte para NWs semiconductores también deben ser más pequeñas que las que caracterizan a sus equivalentes volúmicos $[30,34,40,69]$, por lo que su valor de nuevo se determina a través de un ajuste a lo datos experimentales.

Estas condiciones que deben verificar tanto las velocidades del sonido como las temperaturas de corte, se ve satisfecha por los valores encontrados para ellas del ajuste, mostrando que en todos los casos son menores que los valores obtenidos para el silicio volúmico.

Hasta este momento sabemos que el modelo construido permite obtener buenos ajustes a los datos experimentales, pero para ello se requiere del ajuste de cuatro parámetros libres que se determinan por ajuste a los datos experimentales. Esto implica que por ahora este modelo sólo resulta útil para predecir la conductividad térmica en NWs lisos para temperaturas para las que no hay datos experimentales. Esto puede resultar útil para extender los valores de la conductividad térmica de estos NWs, para los que hay medidas experimentales hasta $320 \mathrm{~K}$, al rango de alta temperatura, ya que el modelo se ha testado en todo el rango de temperaturas para semiconductores volúmicos, y que nada parece indicar que esta capacidad de predecir la conductividad térmica se pierda en los NWs.

Este mismo modelo puede aplicarse para NWs rugosos de silicio; estos NWs tiene las mismas propiedades de cristalinidad y concentración de impurezas que los NWs lisos, ya que muchas veces se obtienen por ataques de la superficie de NWs lisos [22]. Dado que su única diferencia estriba en la morfología de la superficie, la 


\section{TRANSPORTE TÉRMICO EN NWS SEMICONDUCTORES}

acusada reducción en la conductividad térmica debe estar relacionada únicamente con los eventos de scattering de superficie.

En el término de scattering utilizado en nuestro modelo, dado por la ecuación 2.26, el efecto de la rugosidad se evalúa únicamente considerando el valor de RMS de la superficie dado por $\eta$, es decir solo se tiene en cuenta el "tamaño"de la rugosidad. Comúnmente se acepta que para NWs lisos este valor es de $1 \mathrm{~nm}$ [40], mientras que para NWs rugosos este valor puede ser superior a $5 \mathrm{~nm}$ [22]. Cuanto más grande es el valor de $\eta$ menor es la probabilidad de que los fonones sufran un evento especular, acercándonos más al límite difusivo. Ahora bien, tal y como M. Lousier encentró [56], incluso para valores tan pequeños de $\eta$ como $1 \mathrm{~nm}$-i.e. dos tres capas atómicas- la ecuación 2.26 nos lleva muy cerca del límite difusivo. Esto implica que por mucho que aumentemos el valor de $\eta$ no vamos a obtener una gran reducción de la conductividad térmica, dado que el MFP de los fonones ya está cerca del limite de Casimir dado por el diámetro. Sin embargo, sabemos que para NWs rugosos este limite de Casimir se puede violar, siendo el MFP de los fonones en estos NWs mucho menor que el límite clásico dado por la ecuación 2.26 [57]. Sobre esta idea introducimos una modificación a la ecuación 2.26 , de tal modo que se transforma en:

$$
\left(\tau_{B}^{i}\right)^{-1}=\left(\frac{1-e^{-\left(\frac{2 \eta \sin \theta}{v_{i}}\right)^{2}}}{1+e^{-\left(\frac{2 n s i n \theta}{v_{i}}\right)^{2}}}\left(\zeta \times \Lambda_{0}\right)^{-1}+L^{-1}\right) v_{i}
$$

Donde se define un nuevo parámetro $\zeta \in[0,1]$, que modifica el MFP de los fonones en el régimen difusivo que ahora estará dado por $\Lambda^{\prime}=\zeta \times \Lambda_{0}$. Por ahora se introduce como una herramienta de cálculo, pero posteriormente veremos que este parámetro puede relacionarse con las propiedades morfológicas de la superficie. Para comprobar esta hipótesis, vamos se utilizara uno de los NWs rugosos de la referencia [22], el cual tiene las mismas dimensiones que uno de los NWs lisos medidos en [11] para los que ya se ha calculado la conductividad térmica. Para calcular la conductividad térmica de un NW rugoso se deben ajustar cinco parámetros a los datos experimentales, las velocidades del sonido, las temperaturas de corte y el parámetro $\zeta$. Ahora bien, dado que hemos asumido que la reducción en la conductividad térmica se debe únicamente a la acción del scattering de superficie, los 
parámetros intrínsecos al NW como son la temperatura de corte y las velocidades del sonido deben permanecer inalterados con independencia de si se trata de un NW liso o rugoso. Esta hipótesis se confirma en la figura 2.7, donde podemos observar como únicamente actuando sobre $\Lambda_{0}^{\prime}$ obtenemos un perfecto ajuste a los valores experimentales para la conductividad térmica de NWs de silicio rugosos de 115 $\mathrm{nm}$ medido en [2]. Para obtener este resultado el valor de $\eta$ se ha fijado en $4.5 \mathrm{~nm}$, pudiéndose observar que únicamente aumentando este valor y manteniendo $\Lambda_{0}^{\prime}$ en el limite de Casimir no se obtiene una reducción de la conductividad térmica muy significativa con respecto a un NW liso, apenas $1 \mathrm{~W} / \mathrm{mK}$ frente a los $30 \mathrm{~W} / \mathrm{mK}$ observados experimentalmente. Sin embargo, a medida que $\zeta$ se va haciendo más pequeño, y por tanto se reduce el valor de $\Lambda_{0}^{\prime}$, podemos observar como el valor de la conductividad térmica se va reduciendo hasta adaptarse perfectamente a los datos experimentales.

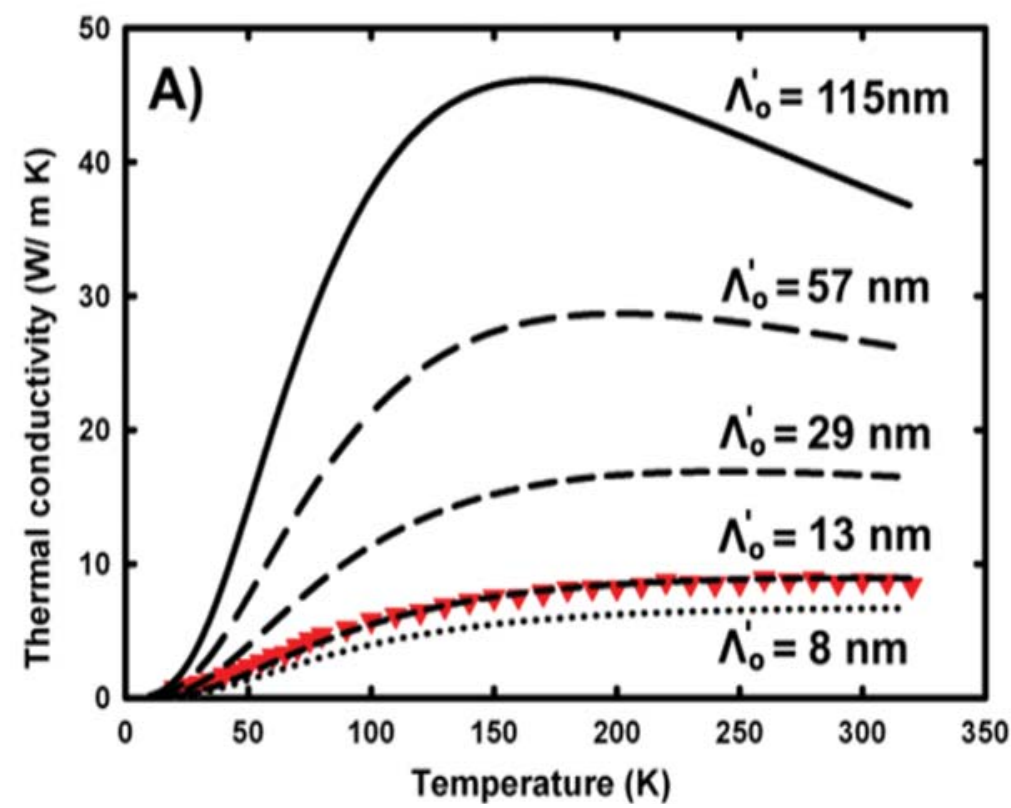

Figura 2.7: Comportamiento de la conductividad térmica en función del parámetro $\zeta$ $-\Lambda_{0}^{\prime}=\zeta \Lambda_{0}$ - que modifica el MFP de los fonones debido a la rugosidad de la superficie de los NWs

Una vez verificado el uso del parámetro $\zeta$ para el cálculo de la conductividad térmica en NWs rugosos y comprobada su validez, podemos calcular la conducti- 


\section{TRANSPORTE TÉRMICO EN NWS SEMICONDUCTORES}

vidad térmica de los NWs rugosos de silicio medidos en las referencias [2] y [22]. Para ello debemos ajustar cinco parámetros a los datos experimentales, los cuales figuran en la tabla 2.3.

\begin{tabular}{|c|c|c|c|c|c|c|c|c|c|}
\hline $\mathrm{D}(\mathrm{nm})$ & $\mathrm{L}(\mathrm{m})$ & $\Lambda_{0}^{\prime}(m)$ & $\eta(m)$ & $\theta_{L}(K)$ & $\theta_{T}(K)$ & $v_{L}(m / s)$ & $v_{T}(m / s)$ & $\gamma_{L}$ & $\gamma_{T}$ \\
\hline 115 & $5 \times 10-6$ & $13 \times 10-9$ & $4.5 \times 10-9$ & 537.5 & 302.9 & 7962 & 4741 & 1.1 & 0.66 \\
\hline 98 & $5 \times 10-6$ & $8.8 \times 10-9$ & $4.5 \times 10-9$ & 530.1 & 300.2 & 7745 & 4584 & 1.1 & 0.66 \\
\hline 50 & $5 \times 10-6$ & $3.3 \times 10-9$ & $4.5 \times 10-9$ & 505.9 & 274 & 7159 & 4160 & 1.1 & 0.66 \\
\hline \hline 79.8 & $5 \times 10-6$ & $12.4 \times 10-9$ & $2.7 \times 10-9$ & 524 & 296 & 7473 & 4385 & 1.1 & 0.66 \\
\hline 77.5 & $5 \times 10-6$ & $15.8 \times 10-9$ & $2.3 \times 10-9$ & 523 & 295 & 7440 & 4370 & 1.1 & 0.66 \\
\hline 69.7 & $5 \times 10-6$ & $6.9 \times 10-9$ & $4.3 \times 10-9$ & 517 & 291 & 7340 & 4290 & 1.1 & 0.66 \\
\hline \hline 77.9 & $5 \times 10-6$ & $34.6 \times 10-9$ & $2.9 \times 10-9$ & 523 & 295 & 7445 & 4372 & 1.1 & 0.66 \\
\hline 71 & $5 \times 10-6$ & $16.2 \times 10-9$ & $3.1 \times 10-9$ & 520 & 292 & 7363 & 4309 & 1.1 & 0.66 \\
\hline 70 & $5 \times 10-6$ & $11.2 \times 10-9$ & $2.8 \times 10-9$ & 519 & 291.5 & 7351 & 4305 & 1.1 & 0.66 \\
\hline
\end{tabular}

Cuadro 2.3: Parámetros utilizados en el cálculo de la conductividad térmica de los NWs rugosos de silicio medidos en las referencias [2] y [22].

Los resultados del cálculo de la conductividad térmica para estos NWs rugosos de silicio se muestran en la figura 2.8, donde se puede observar el excelente ajuste a los datos experimentales en todo el rango de temperaturas para el que está medida la conductividad térmica.

\subsubsection{Modelo predictivo para la conductividad térmica de NWs de silicio lisos}

El modelo desarrollado hasta el momento permite reproducir de forma precisa la conductividad térmica de NWs lisos y rugosos, pero sus cualidades predictivas se limitan únicamente a la extensión en temperatura de la conductividad térmica de los NWs para los que existen medidas experimentales. La obtención de dichas medidas resulta muy compleja y laboriosa. Esto, unido al hecho de que la conductividad de cada NW depende de sus características morfológicas, hace que el desarrollo de una herramienta capaz de predecir la conductividad térmica en función del diámetro y de la morfología de la superficie sea en extremo interesante.

En este apartado veremos como a través de nuestro modelo podemos conseguir esta capacidad predictiva para NWs lisos en nuestro rango de interés; es decir, para NWs con diámetros comprendidos entre 25 y algo más de $100 \mathrm{~nm}$. 

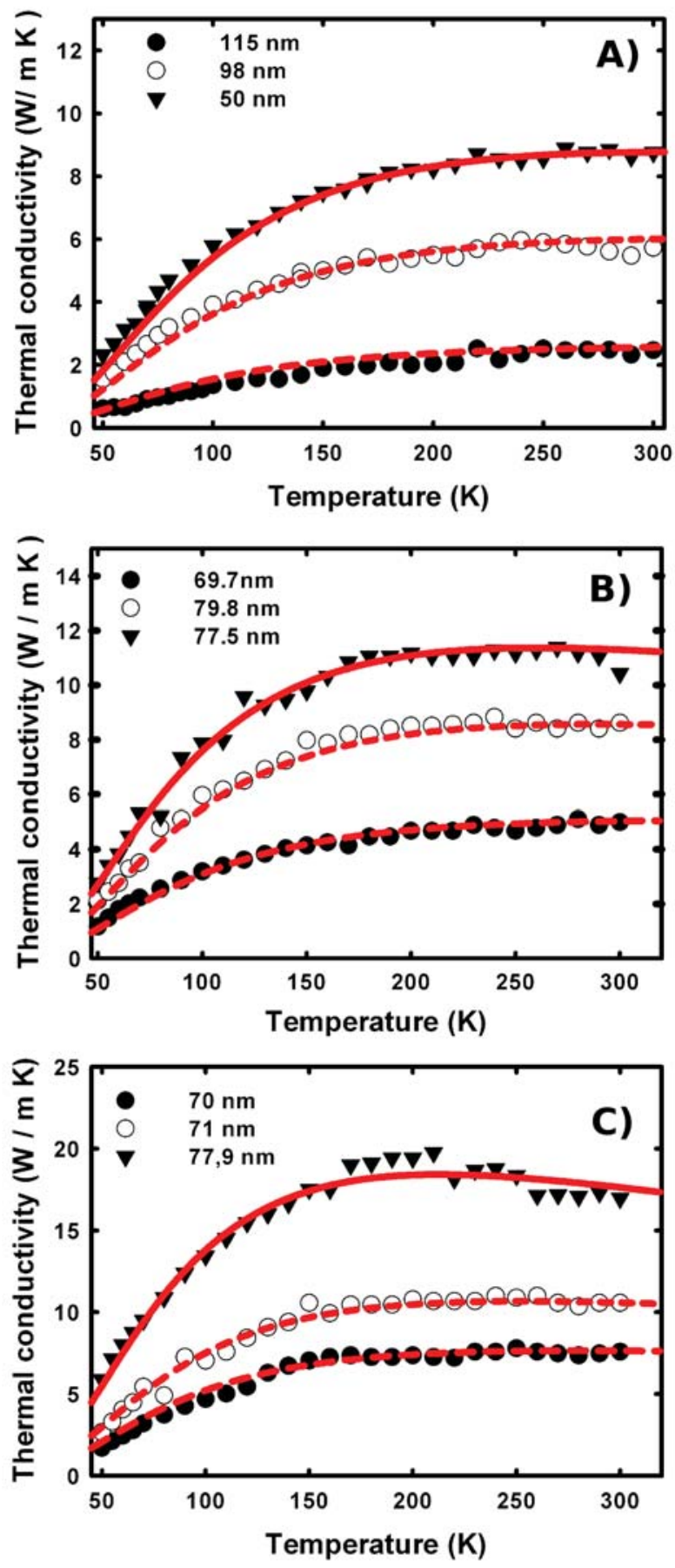

Figura 2.8: Conductividades térmicas experimentales de NWs de silicio rugosos obtenidas de las referencias $[2](A)$ ) y [22] $(B)$ y $C$ ) (símbolos rojos) y conductividades térmicas calculadas con nuestro modelo para estos NWs con los parámetros de la tabla 2.3 (lineas) 


\section{TRANSPORTE TÉRMICO EN NWS SEMICONDUCTORES}

Para determinar la conductividad térmica de un NW de silicio liso se necesita ajustar los valores de cuatro parámetros, las dos velocidades del sonido y las dos temperaturas de corte correspondientes a las ramas transversal y longitudinal acústica. Si estos valores estuviesen relacionados de alguna manera con el diámetro del NW obtendríamos un modelo en que la conductividad térmica quedaría determinada conociendo únicamente el diámetro de dicho NW. Desafortunadamente, sólo disponemos de datos referidos a 4 NWs de silicio lisos, de los cuales uno de ellos está en el límite de validez del modelo $(22 \mathrm{~nm})$. Sin embargo, las conductividades térmicas de los NWs rugosos se han obtenido con un muy buen acuerdo a los datos experimentales asumiendo que toda la diferencia con los NWs lisos recae en el scattering debido a la superficie, y por tanto asumiendo que los valores de las temperaturas de corte y las velocidades del sonido son iguales para NWs rugosos y NWs lisos. Utilizando esto, la población de hilos de distinto diámetro aumenta lo suficiente como para buscar una posible relación entre estos parámetros y las dimensiones del NW.

En la figura 2.9 se han representado los valores de estos parámetros en función de los diámetros de sus respectivos NWs. Como podemos observar en todos los casos aparece un tendencia clara que indica una dependencia con el diámetro, pudiéndose ajustar mediante una regresión lineal con $R^{2}>0,995$ en todos los casos.

Concretamente, para NWs entre 20 y 120 nm de diámetro las velocidades del sonido tienen una dependencia cuadrática con el diámetro dada como:

$$
v_{i}=v_{i}^{0}+a_{1}^{i} \times D^{2}
$$

Mientras que las temperaturas de corte se pueden ajustar a una función racional algo más compleja dada por:

$$
\theta_{i}=\frac{1+b_{1}^{i} D}{b_{2}^{i}+b_{3}^{i} D}
$$

Los valores de los parámetros que aparecen en ambas formulas se resumen en la tabla 2.4:

La determinación fenomenológica de las relaciones entre los parámetros libres y el diámetro de cada NW nos permite predecir la conductividad térmica para cual- 

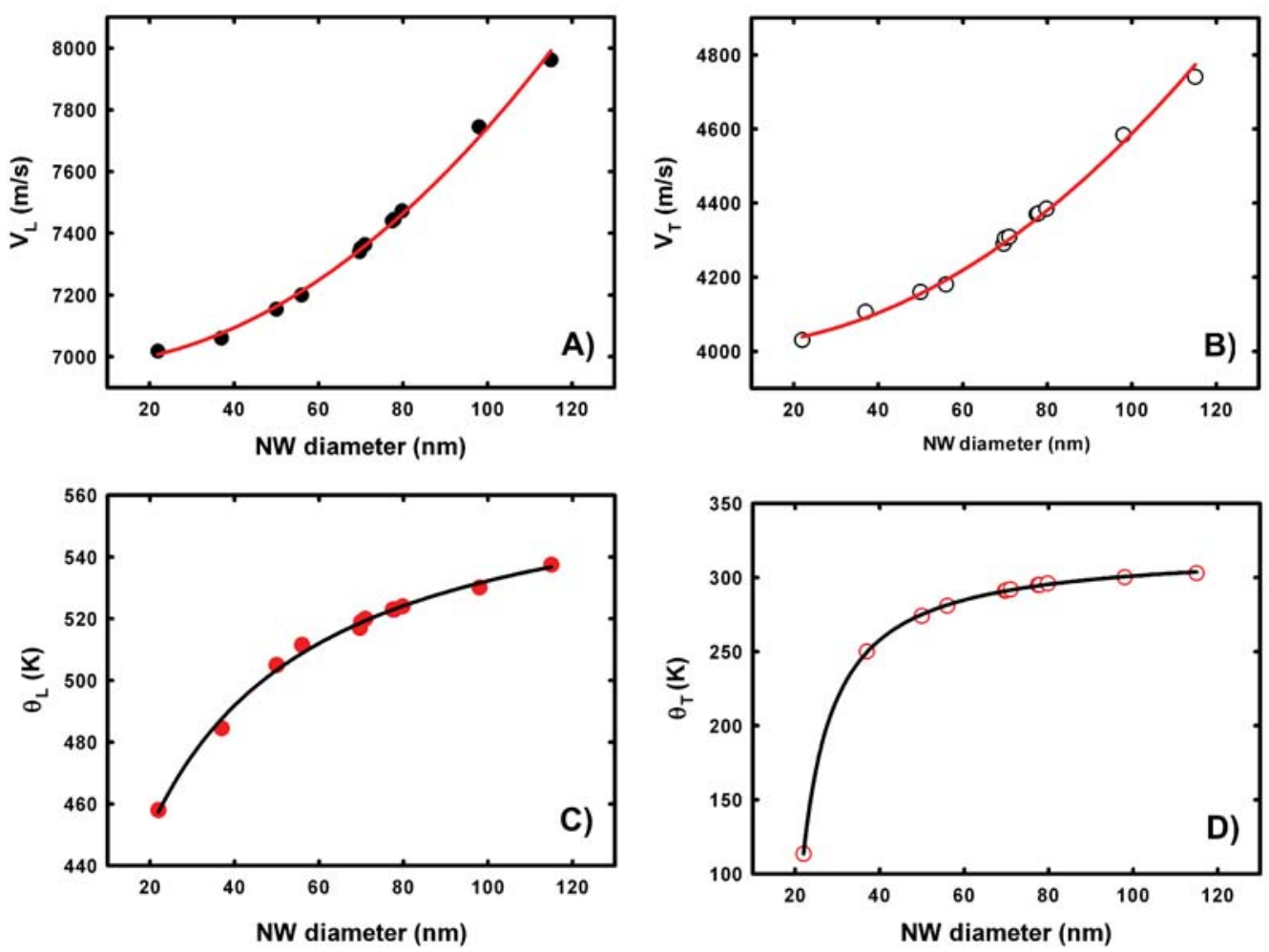

Figura 2.9: Velocidades del sonido de las bandas transversal $\left.\left(v_{t}, \mathrm{~A}\right)\right)$ y longitudinal $\left.\left(v_{L}, \mathrm{~B}\right)\right)$ en función del diámetro del NW. Temperaturas de corte de las bandas transversal $\left.\left(\theta_{t}, \mathbf{C}\right)\right)$ y longitudinal $\left.\left(\theta_{L}, \mathbf{D}\right)\right)$ en función del diámetro del NW.

\begin{tabular}{|c|c|c||c||c|c|c|c|}
\hline & $v_{i}^{0}$ & $a_{1}^{i}$ & & & $b_{1}^{i}$ & $b_{1}^{i}$ & $b_{1}^{i}$ \\
\hline$v_{L}$ & 6970 & $7.719 \times 10^{-2}$ & & $\theta_{L}$ & $8.498 \times 10^{-2}$ & $3.011 \times 10^{-3}$ & $1.483 \times 10^{-4}$ \\
\hline$v_{T}$ & 4020 & $5.644 \times 10^{-2}$ & & $\theta_{T}$ & $-5.190 \times 10^{-2}$ & $2.324 \times 10^{-3}$ & $-1.624 \times 10^{-4}$ \\
\hline
\end{tabular}

Cuadro 2.4: Parámetros obtenidos de los ajustes de las curvas mostradas en la figura 2.9 . 


\section{TRANSPORTE TÉRMICO EN NWS SEMICONDUCTORES}

quier NW liso; siempre y cuando, dicho diámetro se encuentre en, ó próximo al rango especificado para la validez de la parametrización.

La forma de la conductividad térmica, tanto para NWs lisos como para NWs rugosos, es muy dependiente de los valores que toman las velocidades del sonido y de las temperaturas de corte para cada rama fonónica. Esto implica que si se calcula la conductividad térmica con unos valores ligeramente erróneos para estos parámetros, las curvas obtenidas no seguirán la tendencia marcada por los datos experimentales en función de la temperatura, y que la desviación se manifestará de forma muy evidente. Teniendo esto en mente, y dado que no tenemos más datos experimentales fiables para NWs de silicio lisos con los que testar el modelo, la validación de esta aproximación se realiza sobre una serie de datos experimentales procedentes de la referencia [22]. Estas medidas corresponden a NWs rugosos de distintos diámetros y únicamente para tres temperaturas, 100, 200 y 300 Kelvin; conviene recordar que en nuestra aproximación los valores de las temperaturas de corte y las velocidades del sonido son idénticos para un NW con un determinado diámetro, independientemente de sí éste es liso ó rugoso; por tanto, sí obtenemos un buen ajuste a los datos experimentales a baja, media y alta temperatura, calibrando únicamente el efecto de la superficie en una temperatura, podremos validar la aproximación.

En la figura 2.10 podemos ver el resultado de nuestro modelo, donde únicamente se ha tenido que ajustar el parámetro $\zeta$ de la ecuación 2.29 para uno de los puntos en cada caso, en concreto la conductividad térmica a $300 \mathrm{~K}$. Puede observarse como las curvas obtenidas para la conductividad térmica pasan automáticamente por los valores experimentales de la conductividad térmica a 100 y $200 \mathrm{~K}$ en todos los casos, demostrando que nuestra aproximación fenomenológica ofrece un resultado muy bueno para predecir la conductividad térmica en NWs de silicio.

El modelo desarrollado hasta este punto es capaz de predecir la conductividad térmica para NWs de silicio lisos sin ningún parámetro de ajuste; por tanto, conociendo únicamente el diámetro se puede generar de forma precisa toda la curva de la conductividad térmica en función de la temperatura (Figura 2.11). Por otro lado, también somos capaces de predecir la conductividad térmica de NWs de silicio rugosos en todo el rango de temperaturas conociendo únicamente un valor experimental de la conductividad térmica a una temperatura cualquiera. Esto simplifica 


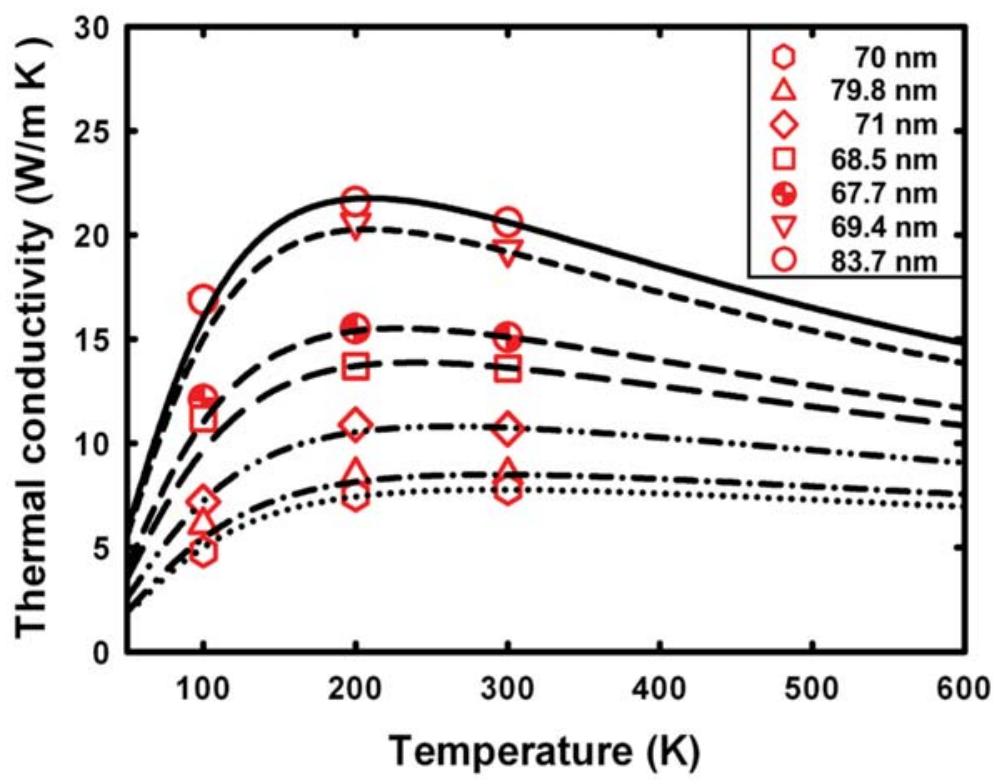

Figura 2.10: Conductividad térmica experimental para diversos NWs de silicio de la referencia [22] y curvas predichas para la conductividad térmica mediante nuestra aproximación predictiva.

mucho la labor experimental, dado que únicamente hay que medir la conductividad térmica para una temperatura en vez de tener que barrer experimentalmente todo el rango de temperaturas para cada NW rugoso. Pese a que esto ya constituye un gran avance frente a lo aportado por otros modelos, aún puede conseguirse un mayor avance estudiando el trasfondo del parámetro $\zeta$, tal y como veremos en el siguiente apartado.

Por otro lado, si representamos la conductividad térmica de los NWs de silicio en función del diámetro de los mismos, y para una temperatura dada, obtenemos unas curvas suaves que pueden ajustarse perfectamente en el rango de validez, $R^{2}>$ 0,999, a unas funciones del tipo:

$$
\kappa=g_{0}+g_{1} \log (D)
$$

Esto nos permite ofrecer una expresión mucho más simple que la evaluación completa de 2.16 para la conductividad térmica a una temperatura determinada; 


\section{TRANSPORTE TÉRMICO EN NWS SEMICONDUCTORES}

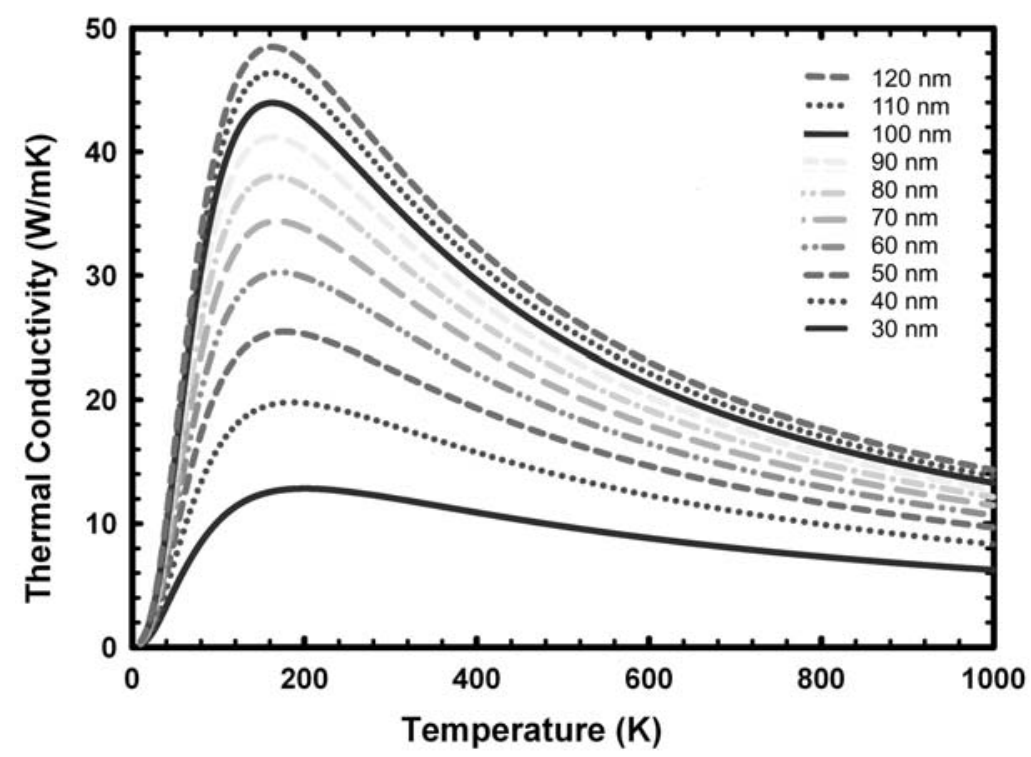

Figura 2.11: Conductividades térmicas calculadas para NWs de silicio lisos en un amplio rango de diámetros mediante nuestro modelo predictivo

por ejemplo, la conductividad térmica de los NWs de silicio lisos con diámetros comprendidos desde $25 \mathrm{~nm}$ a algo más de $120 \mathrm{~nm}$ a un temperatura de operación típica de $300 \mathrm{~K}$ se puede obtener a través de:

$$
\kappa^{300 K}=-56,4+20,1 \log (D)
$$

\subsubsection{Modelo predictivo para la conductividad térmica de NWs de silicio} rugosos

El efecto introducido por las paredes de los NWs en el transporte térmico está descrito en nuestro modelo a través de la ecuación 2.29. En ella, el papel jugado por los parámetros que caracterizan la morfología de la superficie está únicamente representado de forma explicita por el valor RMS de la superficie o $\eta$. Este parámetro aparece en la probabilidad de evento especular $p$, y como hemos visto su efecto no es muy determinante, dado que, incluso para los NWs considerados como lisos, esta probabilidad es muy cercana a cero. Es decir, mayoritariamente suceden eventos 


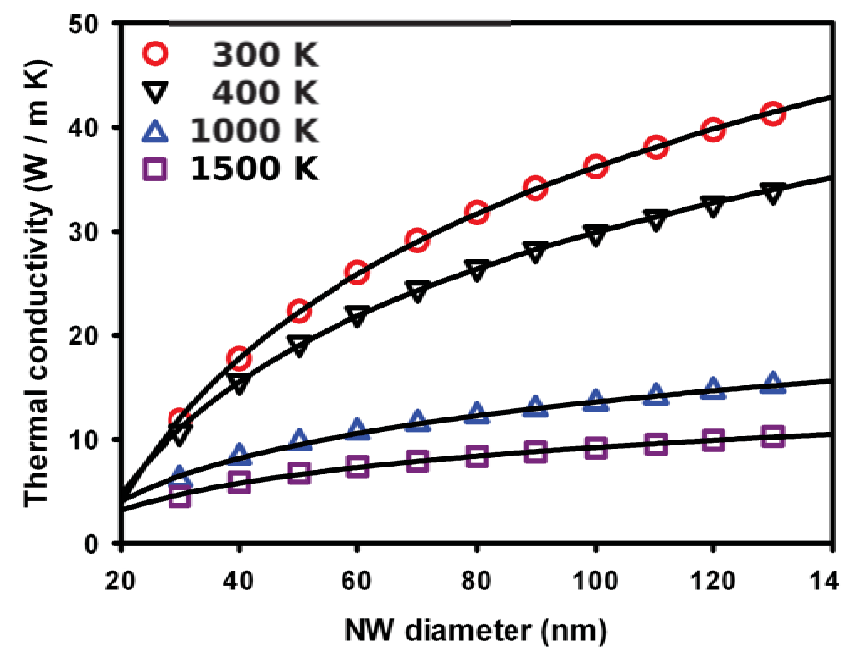

Figura 2.12: Conductividades térmicas a 300 y $400 \mathrm{~K}$ calculadas para NWs de silicio lisos en función del diámetro en el rango de validez del modelo mediante nuestro modelo predictivo (símbolos). Las curvas corresponden al ajuste ajuste dinámico a la expresión 2.32

difusivos que confinan el MFP fonónico cerca, o incluso por debajo, del límite de Casimir dado por el diámetro. Sin embargo, existen claras evidencias de que para considerar completamente el efecto de la superficie en el transporte térmico de los NWs, el término de scattering de superficie debe incluir el efecto de la longitud de correlación de las rugosidades así como su tamaño medio $\eta[22,57]$.

Con nuestro modelo se consigue un magnifico ajuste a los datos experimentales para NWs rugosos procedentes de varios autores a través de una modificación al termino de scattering desarrollado por J.M. Ziman, consistente en introducir un parámetro $\zeta$ que da cuenta de la reducción del MFP de los fonones por efecto de la superficie. Este parámetro se ha determinado para cada NW por ajuste a los datos experimentales a una temperatura, pero como podremos comprobar $\zeta$ va a ser más que un mero parámetro de ajuste.

La dependencia de la conductividad térmica de los NWs de silicio rugosos en función de los parámetros característicos de la superficie, $\eta$ y longitud de correlación, ha sido experimentalmente comprobada por J. Lim et al. [22]. En dicho trabajo, más de 25 NWs de silicio, con diferentes diámetros y características superficiales, aparecen estudiados tanto a nivel morfológico como de su conductividad térmica 


\section{TRANSPORTE TÉRMICO EN NWS SEMICONDUCTORES}

para tres temperaturas diferentes (100, 200 y 300 K). Dicho artículo demuestra que al representar la conductividad térmica en función del diámetro, o en función del tamaño de la rugosidad $\eta$, o de la longitud de correlación, exclusivamente en cada caso, no aparece una correlación evidente. Esto indica que la conductividad térmica es fuertemente dependiente de una combinación inseparable de todas ellas. Sin embargo, los esfuerzos realizados por encontrar dicha correlación en [22] dejan fuera el efecto del diámetro, y por ello solo consiguieron encontrar una correlación débil y de forma muy indirecta.

En nuestra investigación, estos mismos datos procedendentes de [22] que caracterizan experimentalmente conductividades térmicas, $\eta^{\prime} s$ y longitudes de correlación, van a utilizarse para estudiar en profundidad la naturaleza del parámetro $\zeta$ utilizado en nuestro modelo.

Se sabe que la conductividad térmica en los NWs de silicio lisos es fuertemente dependiente del diámetro de los mismos [11]. Esta dependencia se refleja principalmente en la dependencia de las velocidades del sonido y temperaturas de corte de los NWs con su diámetro dadas por 2.30 y 2.31, además de en el termino de scattering de superficie, en el cual el diámetro supone el límite para el MFP de los fonones a través del límite de Casimir dado por $\Lambda_{0}=D$. En el caso de los NWs rugosos, las velocidades del sonido y las temperaturas de corte tienen la misma dependencia con el diámetro que la que presentan los NWs lisos; sin embargo, el MFP de los fonones en estos NWs rugosos es menor que el dado por el diámetro del NW, estando caracterizado en nuestro marco de trabajo por $\Lambda_{0}^{\prime}=\zeta \times \Lambda_{0}$. Cuando representábamos la conductividad térmica de los NWs lisos en función del diámetro para una temperatura dada (Figura 2.12), podíamos observar como se obtenía una curva suave que simplificaba la obtención de la conductividad térmica para cualquier NW en un amplio rango de diámetros. Ahora bien, si se construye la misma gráfica para NWs rugosos de silicio, la conductividad térmica en función del diámetro aparece retratada como una nube de puntos sin orden aparente (Figura 2.13 A) ). Si en vez de representar estas conductividades térmicas frente al diámetro de los NWs lo hacemos frente a $\zeta \times D$, donde $\zeta$ se obtiene de ajustar nuestro modelo a los datos experimentales, podemos observar como la misma nube dispersa de datos mostrada en la figura 2.13 A) se ordena perfectamente en la figura 2.13 B), mostrando un correlación clara y directa con $\zeta$. 
Recordemos que $\zeta$ aparece dentro de una compleja ecuación integral, por lo que este resultado no es para nada trivial y nos permite una primera hipótesis de trabajo; vamos a asumir que $\zeta$ no es solo un parámetro de cálculo, sino que va a ser una magnitud que engloba completamente el efecto de la superficie y que depende fuertemente de los parámetros característicos de la morfología de dicha superficie, dados por $\eta$ y $L_{\text {correl. }}$; aunque también puede tener una dependencia con el diámetro tal y como sugieren P. Martin et al. en [52]. A la vista de figura 2.13 B) la dependencia con $D$ de $\zeta$ es débil, al menos para estos diámetros mucho mayores que el valor de $\eta$. Además podemos observar en 2.13 B) que esta correlación no es casual para $300 \mathrm{~K}$, ya que se mantiene para el resto de temperaturas para los que tenemos datos experimentales (100 y $200 \mathrm{~K}$ ), apareciendo de nuevo una perfecta ordenación de los datos cuando se representan en función de $\zeta \times D$.

Las representaciones de la conductividad térmica en función de $\zeta \times D$ para los NWs rugosos recuerdan mucho en su forma a las representación de la conductividad térmica frente al diámetro dadas en la figura 2.13, pudiéndose asimilar el valor de $\zeta \times D$ a una especie de diámetro efectivo del NW rugoso. Por otro lado, esto demuestra el papel dominante que juega la rugosidad de los NWs en la conductividad térmica de los NWs. Para NWs donde el MFP de los fonones cae por debajo del límite de Casimir, la conductividad térmica puede ajustarse con una $R^{2}>0,99$ a una familia de curvas dada por:

$$
\kappa=g_{1}(\zeta D)^{g_{2}}
$$

Donde $g_{i}$ dependen de la temperatura. Esto permite expresar la conductividad térmica de un NW de silicio rugoso en función de $\zeta$ y el diámetro, a una temperatura de $300 \mathrm{~K}$ de una forma muy simple a través de:

$$
\kappa=1,227(\zeta D)^{0,777}
$$

Por otro lado, podemos eliminar casi completamente el efecto que tiene el diámetro en la reducción de la conductividad térmica de los NWs rugosos a través de la relación $\kappa_{\text {liso }} / k_{\text {rugoso }}$. Dado que la dependencia de las velocidades del sonido y temperaturas de corte con el diámetro son idénticas para NWs lisos y rugosos, 

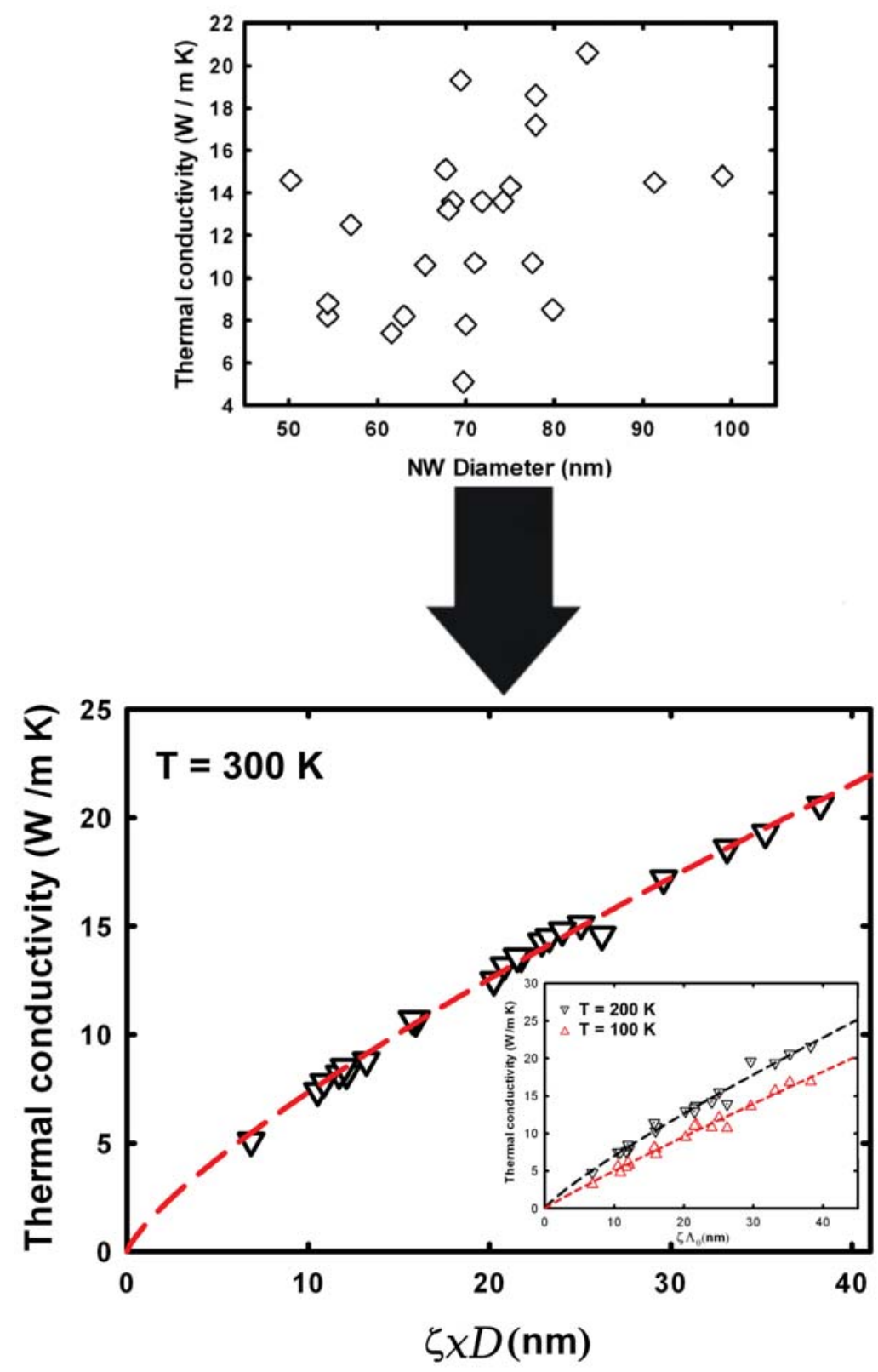

Figura 2.13: Datos experimentales de las conductividades térmicas de NWs con distintas características superficiales en función de su diámetro. Puede observarse una distribución bastante aleatoria de las conductividades térmicas en función del diámetro de los NWs A). En B (puede verse como la nube de puntos mostrada en A) se ordena cuando se representa $\kappa$ en función de $\zeta \times D$. Lo mismo sucede para 100 y 200 $\mathrm{K}$, ver recuadro interior. 
este cociente entre la conductividad térmica de un NW de un determinado diámetro y su homologo de superficie rugosa dependerá casi exclusivamente de $\eta$ y $L_{\text {correl. }}$, y por tanto de $\zeta$. Podemos observar la confirmación de esta hipótesis en la figura 2.14. En concreto cuando se representa $\kappa_{\text {liso }} / \kappa_{\text {rugo }}$ frente a $\zeta^{-1}$, obtenemos una dependencia cuasi-lineal de este cociente con el inverso de $\zeta$.

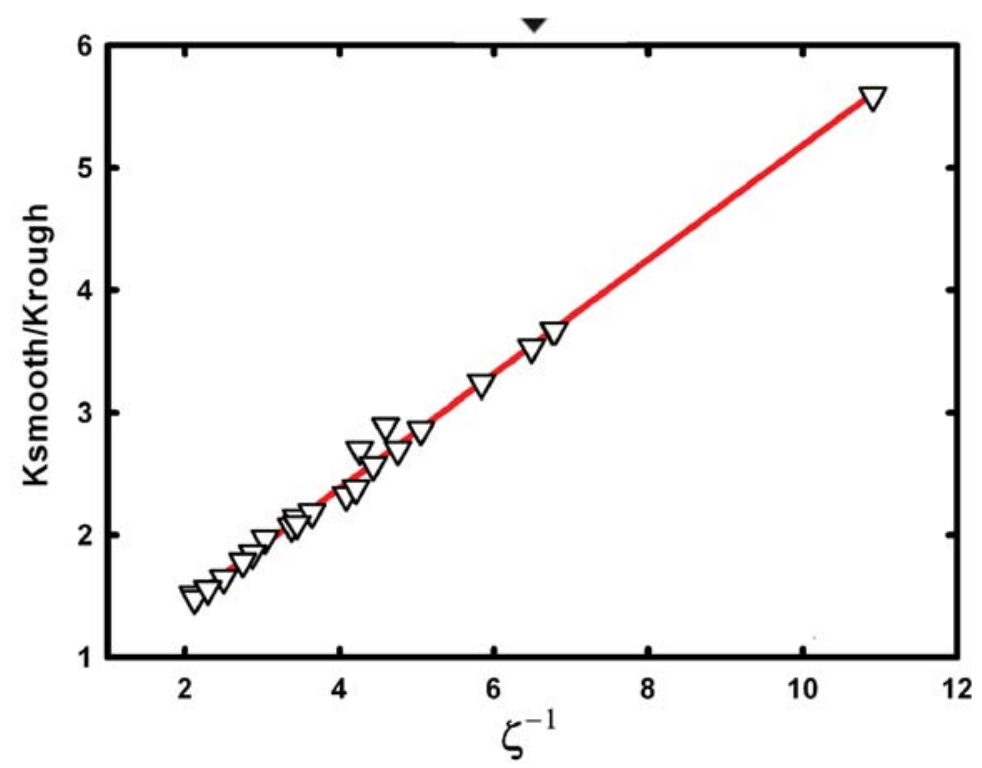

Figura 2.14: Cociente $\kappa_{\text {liso }} / \kappa_{\text {rugo }}$ frente a $\zeta^{-1}$, podemos observar la fuerte correlación que aparece entre ambos

El valor de $\kappa_{\text {liso }}$ utilizado en la figura 2.14 se ha obtenido de nuestro modelo predictivo, que recordemos que para NWs lisos solo requiere del diámetro para calcular de forma precisa la conductividad térmica. Del ajuste por mínimos cuadrados a los puntos que aparecen en la figura 2.14, y con una $R^{2}>0,998$, podemos obtener una ecuación simple que nos relaciona la conductividad térmica de un NW de silicio rugoso con la de su homologo liso y con $\zeta$ para $300 \mathrm{~K}$ :

$$
\kappa_{\text {rugosos }}^{300 K}=\left(\frac{2 \zeta}{1+\zeta}\right) \kappa_{\text {lisos }}^{300 K}
$$

A la vista de los resultados mostrados en las figuras 2.13 B) y 2.14 , nuestra aproximación de que todo el efecto de la superficie está englobado en $\zeta$ resulta 


\section{TRANSPORTE TÉRMICO EN NWS SEMICONDUCTORES}

muy plausible, y por tanto podemos establecer que $\zeta$ debe de ser una forma dependiente de los parámetros característicos de la rugosidad de la superficie y en menor medida del diámetro del NW. Por tanto, encontrar la relación $\zeta=f\left(\eta, L_{\text {corre., } D}\right)$ nos permitiría determinar la conductividad térmica para cualquier NW de silicio, liso o rugoso, conociendo únicamente sus características geométricas y morfológicas.

La tarea de determinar la forma de $\zeta$ puede realizarse a través de un análisis fenomenológico de los resultados mostrados en la referencia [22], ya que de todos los NWs para los que se conoce experimentalmente la conductividad térmica (ver figura 2.13 A) ) también existen datos que describen experimentalmente las características morfológicas de su superficie.

En un análisis fenomenológico es fundamental conocer el grado de exactitud de los datos experimentales que se utilizan, dado que el error que incorporan va a influir de forma determinante en las conclusiones que se pueden extraer del estudio. La determinación experimental de las características de la rugosidad superficial, $\eta$ y $L_{\text {corre, }}$, no es una tarea simple y exenta de error experimental. Si uno mira atentamente los datos aportados por J.Lim et al. en [22], existen bastantes indicios de que las medidas de $\eta$ y $L_{\text {corre. }}$ incorporan un grado de incertidumbre considerable; e.g. para dos NWs de diámetros de 65.4 y $69.7 \mathrm{~nm}$, y valores de $\eta$ y $L_{\text {corre. }}$ prácticamente idénticos, la conductividad térmica del más grueso es la mitad que la del más fino. Dado que sus superficies son idénticas, la diferencia solo podría estar marcada por la diferencia de diámetros, pero aquí el más fino tiene una conductividad mucho mayor que el más grueso, lo que contradice lo que sabemos de otras medidas experimentales y modelos teóricos. Este es un comportamiento en extremo anómalo que solo puede justificarse si los valores de $\eta$ y $L_{\text {corre. }}$ no son "idénticos" para ambos NWs. En otro ejemplo, dos NWs de 54.4 y $79.8 \mathrm{~nm}$ de diámetro tienen una conductividad térmica similar, lo que solo se explicaría si el más grueso fuese también más rugoso; sin embargo, ambos tienen un $L_{\text {corre. }}$ similar mientras que $\eta$ es mayor para el más delgado, lo que de nuevo contradice lo que sabemos del comportamiento de la conductividad térmica en NWs.

Dado que en [22] las conductividades térmicas se han determinado con un método contrastado, el cual no es susceptible de introducir una incertidumbre tal que justifique las anomalías anteriores, estas deben recaer en la determinación experimental 
de $\eta$ y $L_{\text {corre. }}$.

El valor de $\eta$, o la altura media de la rugosidad, se obtiene en [22] a través de imágenes de alta resolución TEM de cada NW. Los NWs se dividen en secciones de $500 \mathrm{~nm}$ sobre las que se extrae la rugosidad media, siendo el valor final de $\eta$ el resultante de hacer la media de el valor obtenido en cada sección. Esta medida se realiza en una proyección 2D del NW (la dada por la imagen TEM), y por tanto se está extrapolando a toda la superficie lo observado en una pequeña porción de la misma. El error reportado en [22] para $\eta$ puede alcanzar los $\pm 0,6 \mathrm{~nm}$ en algunos NWs, no correspondiendo necesariamente este valor con el NW con $\eta$ más grande o mayor diámetro; por tanto, dadas las limitaciones del método de medida, vamos a considerar por seguridad un valor para el error de $\eta$ dado por este limite para toda la muestra.

Por otro lado, el proceso de determinación de $L_{\text {corre. }}$ es un proceso más delicado. En [22] el valor de $L_{\text {corre }}$. se extrae de las imágenes TEM de alta resolución de cada NW; de estas imágenes se extrae el espectro de potencia de la rugosidad a través de una transformada rápida de Fourier (FFT) del perfil de la superficie, asumiendo para ello una función de autocovarianza exponencial [22].

La evaluación de $L_{\text {corre. }}$ en una superficie rugosa mediante el método adoptado en [22] encierra cierta controversia. S.M. Goodnick en [71] demuestra que la determinación de $L_{\text {corre }}$ directamente de la FFT del perfil de superficie no es el método más preciso. Posteriormente G. Rasigni demostró que una función exponencial no cumple los requisitos para ser considerada una función de autocorrelación valida cundo se trabaja en superficies rugosas [72], teniendo que recurrirse a otro tipo de funciones de autocorrelación de tipo gaussiano o lorentziano por ejemplo. La discrepancia del valor de $L_{\text {corre }}$ obtenido a partir de distintas funciones de autocorrelación, como por ejemplo de una exponencial o de una gaussiana, puede ser superior al $30 \%$ del valor de $L_{\text {corre. }}[71,72]$. Por tanto, los datos de $L_{\text {corre }}$ que aparecen en [22] son susceptibles de tener una incertidumbre dada por el método escogido para su determinación de hasta el $30 \%$ de su valor.

El error nada despreciable que envuelve los valores experimentales de $\eta$ y $\mathbf{Ł}_{\text {corre. }}$ dificulta bastante la determinación fenomenológica de $\zeta$ a partir de dichos valores. Pero dado que contamos con datos de más de $25 \mathrm{NWs}$ diferentes provenientes de [22], esta incertidumbre en la medida se puede reducir estadísticamente gracias a la 


\section{TRANSPORTE TÉRMICO EN NWS SEMICONDUCTORES}

elevada población de datos disponibles.

A la hora de describir la dependencia de $\zeta \operatorname{con} \eta, L_{\text {corre. }}$ y $D$, deben verificarse ciertos límites físicos y comportamientos que emanan de la observación de los datos experimentales para la conductividad térmica de NWs de silicio:

- El valor de $\zeta$ depende del diámetro; una determinada morfología superficial afectará más a NWs delgados que a NWs más gruesos, pero su efecto no es muy importante para valores de $\eta / D / 2$ pequeños, de modo que si $\eta \sim(D / 2)$ la conductividad térmica tiende a cero.

- El valor de $\zeta \in[0,1]$, se aproxima a 1 cuando el NW puede ser descrito como liso i.e. $\eta \sim 1 n m$ y $L_{\text {corre. }}$ es grande en comparación.

- El valor de $\zeta$, tiende a 1 cuando $L_{\text {corre. }}$ tiende a infinito y $\eta<<D / 2$.

- $\eta$ y $L_{\text {corre. }}$ están estrechamente relacionadas, de modo que si para un mismo NW aumenta el valor de $\eta$ manteniedose el de $L_{\text {corre. }}$ la conductividad térmica disminuye, del mismo modo si $\eta$ se mantiene pero $L_{\text {corre }}$ aumenta, la conductividad térmica del NW aumenta.

Teniendo en cuenta estas condiciones, hemos desarrollado una relación fenomenológica para $\zeta$ que verifica todas estas condiciones dada como:

$$
\zeta=\left(1-\frac{\eta-\eta_{0}}{R}\right) e^{-\alpha \frac{\eta}{L_{\text {correlacion }}}}
$$

Donde $\eta_{0}=1 \mathrm{~nm}$ es el valor del límite liso y el valor del parámetro $\alpha=3,4$ lo obtenemos del mejor ajuste a los datos experimentales de la referencia [22]. La validación de esta aproximación la realizamos introduciendo esta formula en la expresión 2.29 y calculando mediante la ecuación integral 2.17 la conductividad térmica a $300 \mathrm{~K}$ para todos los NWs de silicio que aparecen en [22], dejando como únicos parámetros libres $\eta$ y $L_{\text {correlacion. }}$ Si la aproximación es válida debería aparecer una correlación lineal de pendiente 1 entre estos $\eta$ y $L_{\text {correlacion }}$ obtenidos por ajuste y $\operatorname{los} \eta$ y $L_{\text {correlacion }}$ medidos experimentalmente en la referencia [22]. En las figuras 15 A) y 15 B). se ha representado la relación entre estos valores procedentes del ajuste y los valores experimentales. Puede observarse como aparece una correlación de estos parámetros con el comportamiento esperado, siendo la diferencia con 
el ideal justificada plenamente con los errores experimentales atribuibles al proceso de determinación experimental de $\eta$ y $L_{\text {correlacion }}$ llevados a cabo en [22]. Una vez
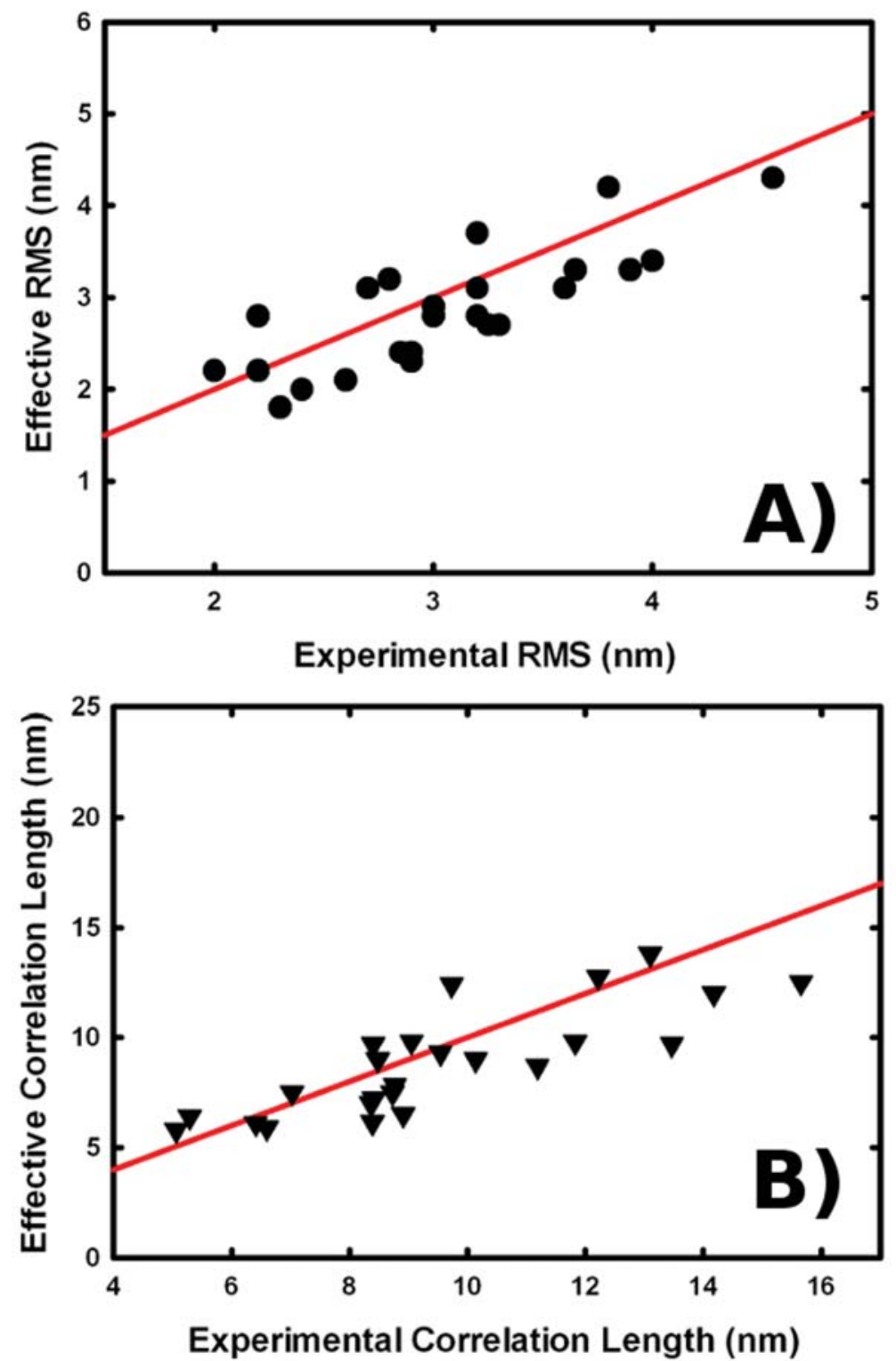

Figura 2.15: Representación de los valores teóricos de RMS o $\eta$ frente a los valores experimentales de $\eta \mathrm{A}$ ) y Representación de los valores teóricos de $L_{\text {correlacion }}$ frente a los valores experimentales de $L_{\text {correlacion }}$ B). Los datos experimentales proceden de [22]. Puede observarse en ambos casos una buena correlación lineal de pendiente 1 


\section{TRANSPORTE TÉRMICO EN NWS SEMICONDUCTORES}

corroborada nuestra formula fenomenológica para $\zeta$, el modelo construido para la conductividad térmica de NWs de silicio es completamente predictivo, dependiendo únicamente de las variables intrínsecas de cada NW dadas por su geometría y morfología superficial. Además igual que hicimos para los NWs lisos, donde se dio una expresión simplificada para la conductividad térmica a $300 \mathrm{~K}$, podemos dar un ecuación simplificada para la conductividad térmica en NWs rugosos:

$$
\kappa=1,23\left(\left(1-\frac{\eta-\eta_{0}}{R}\right) e^{-3,4 \frac{\eta}{L_{\text {correlacion }}} D}\right)^{0,78}
$$

\subsubsection{Modelo para la Conductividad térmica de NWs de silicio-germanio}

El cálculo de la conductividad térmica en aleaciones semiconductoras como el silicio-germanio $\left(S i_{1-X} G e_{X}\right)$ es una tarea más complicada que para semiconductores puros. La principal diferencia estriba en el efecto que la aleación tiene en el transporte fonónico, dado que los átomos de germanio aparecen como fuertes centros de scattering para la componente fonónica perteneciente al silicio y viceversa. Para calcular la conductividad térmica de la aleación de $S i_{1-X} G e_{X}$ utilizaremos nuestro modelo para la conductividad térmica, pero adecuadamente modificado para responder a las condiciones impuestas por la aleación.

Trabajando en la hipótesis introducida por Z. Wang Z y N. Mingo [60], vamos a considerar que todos los eventos de scattering de cada elemento son independientes entre sí, salvo el correspondiente a los eventos debidos a las impurezas de la red. De este modo los términos correspondientes a los tiempos de relajación debidos a los eventos normales, umklapp y de superficie se compondrán genéricamente como:

$$
\tau_{S i_{1-X} G e_{X}}^{i}=\left(\frac{X}{\tau_{S i}^{i}}+\frac{1-X}{\tau_{G e}^{i}}\right)^{-1}
$$

Donde cada $\tau$ corresponde genéricamente a los eventos normales, umklapp y de superficie para el silicio y el germanio. Por otro lado las temperaturas de corte y velocidades del sonido del $S i_{1-X} G e_{X}$ también se construyen con una ley de mezclas independientes dada por:

$$
\theta_{S i_{1-X} G e_{X}}^{i}=\left(\frac{X}{\theta_{S i}^{i}}+\frac{1-X}{\theta_{G e}^{i}}\right)^{-1}
$$




$$
v_{S i_{1-X} G e_{X}}^{i}=\left(\frac{X}{v_{S i}^{i}}+\frac{1-X}{v_{G e}^{i}}\right)^{-1}
$$

Por ultimo, el efecto de la aleación es el único considerado no aditivo, existiendo un termino de mezcla o Bowing; dado que su efecto es de la misma naturaleza que la existencia de diferentes isotopos en el cristal, puede introducirse este tiempo de relajación debido a la aleación junto con el termino debido a las impurezas:

$$
\tau_{I}^{S i_{1-X} G e_{X}}=\left(\frac{X}{\tau_{I}^{S i}}+\frac{1-X}{\tau_{I}^{G e}}+X(1-X) C_{\text {Bowing }} \omega^{4}\right)^{-1}
$$

Donde el termino dado por la aleación se ha construido de forma similar al de la referencia [60]. Al igual que se hizo para cristales puros, se ha validado el modelo para la aleación macroscópica de $S i_{1-X} G e_{X}$. Hay que tener en cuenta que los datos experimentales de la conductividad térmica del $S i_{1-X} G e_{X}$ difieren según autores $[73,74]$; la conductividad térmica depende de la homogeneidad de la muestra y probablemente este factor sea el culpable de la discrepancia entre autores. En la figura 2.16 A) y $2.16 \mathrm{~B}$ ) se muestra el resultado de nuestro modelo para las conductividades térmicas del $S i_{1-X} G e_{X}$ experimentales de las referencias [73] y [74] respectivamente, mostrando un excelente acuerdo con los datos experimentales en cada caso.

Dado que los parámetros del $S i_{1-X} G e_{X}$ necesarios para el calculo de la conductividad térmica se calculan a partir de los del silicio y el germanio puros, y que estos ya se han calculado, el modelo solo tiene un parámetro libre, $C_{\text {Bowing }}$. Este parámetro debido a la aleación se ha ajustado para obtener la conductividad térmica para $X=0,1$ (composición que existe experimentalmente tanto en [73] como [74]), obteniéndose el resto de la curva con ese valor. Los valores de $C_{\text {Bowing }}$ son diferentes para los datos de [73] y [74], siendo $C_{\text {Bowing }}{ }^{73}=1,5 \times 10^{-42} \mathrm{y}$ $C_{\text {Bowing }}{ }^{74}=2,8 \times 10^{-42}$. Esto es lo esperable dado que los valores de la conductividad térmica difieren para las mismas composiciones según ambos autores.

Una vez validado el modelo para aleaciones macroscópicas, se puede utilizar para intentar predecir la conductividad térmica de NWs de $S i_{1-X} G e_{X}$. El modelo para la conductividad térmica de la aleación requiere conocer los parámetros de los componentes puros, y aquí surge la principal dificultad; no existen muchos datos experimentales para NWs de germanio, y los pocos que existen corresponden a 

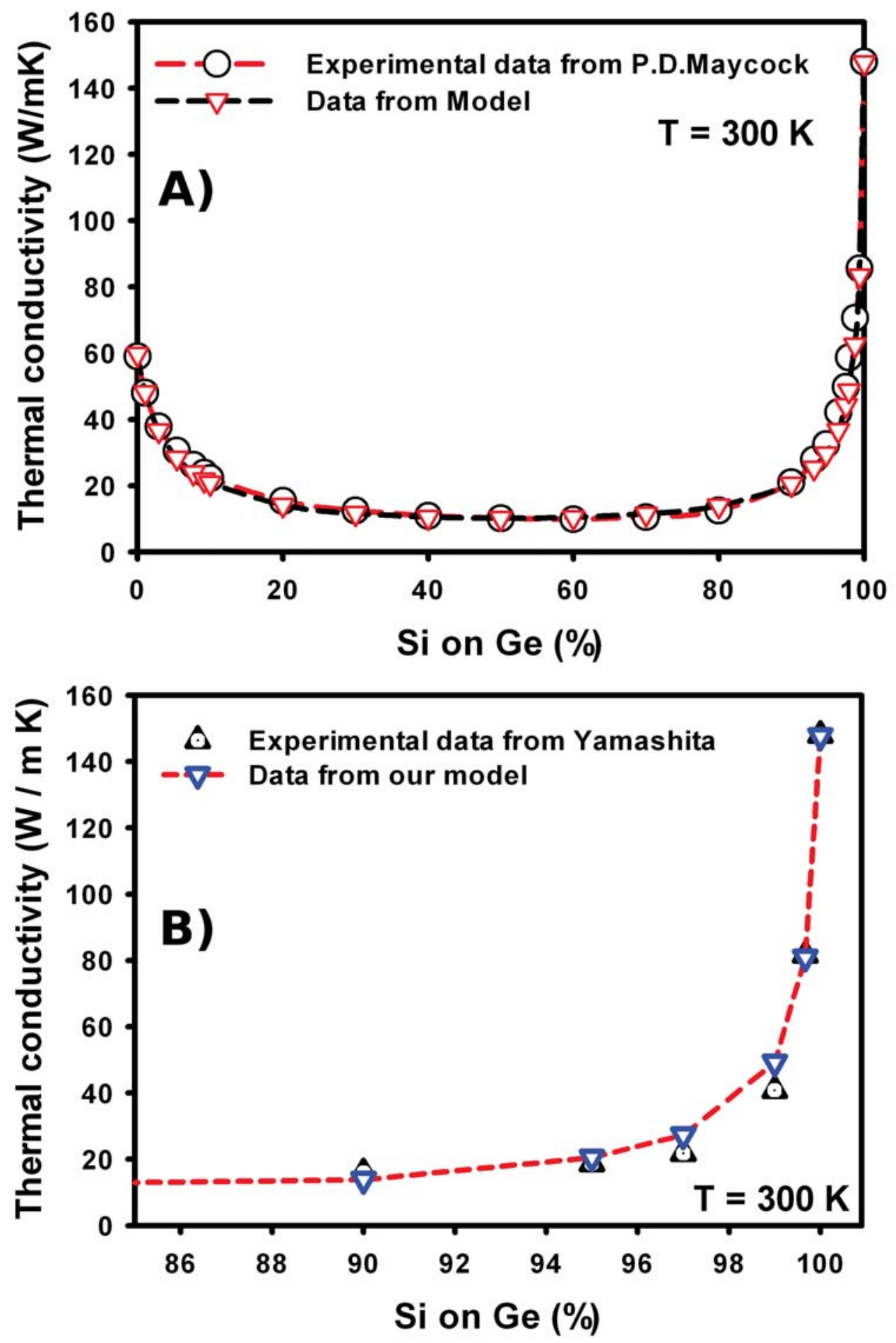

Figura 2.16: Conductividades térmicas experimentales para el $S i_{1-X} G e_{X}$ volúmico en todo el rango de composición a $300 \mathrm{~K}$. Los datos experimentales proceden de la referencia [73], A) y referencia [74] B) 
NWs con diámetros un poco por debajo del límite de aplicación de nuestro modelo (ver Figura 2.2 ). Además de estas medidas experimentales, existen unas predicciones teóricas para NWs de germanio dadas por N. Mingo en [31], utilizando para ello un modelo que al menos entre 200 y $300 \mathrm{~K}$ daba muy buen resultado para NWs de silicio lisos.

Para estimar la conductividad térmica de los NWs de germanio vamos a realizar unas hipótesis de trabajo, y posteriormente compararemos nuestros resultados con los predichos en [31], asumiendo que si mediante dos modelos completamente diferentes, y que funcionan bien para otros materiales similares, se llega a un resultado equivalente, la solución puede tomarse como una buena aproximación a la realidad. Para obtener la conductividad térmica de los NWs de germanio con nuestro modelo es necesario conocer las velocidades del sonido y temperaturas de corte de cada banda. Como no tenemos posibilidad de ajustar a los datos experimentales de diferentes NWs de germanio, vamos a trabajar en la hipótesis de que la dependencia funcional de estos parámetros con el diámetro de los NWs es similar a la encontrada en el silicio. Por simplicidad, vamos a suponer que las funciones que caracterizan las velocidades del sonido y temperaturas de corte para el germanio sólo diferirán en una constante de ajuste de las del silicio; recordemos que estos valores para las temperaturas de corte y velocidades del sonido tienen que ser menores que las del silicio:

$$
\begin{array}{r}
v_{i}^{G e_{N W}}=\chi\left(\frac{v_{i}^{G e_{M a c r}}}{v_{i}^{S i_{M a c r}}}\right)\left(v_{i}^{0}+a_{1}^{i} \times D^{2}\right)^{S i_{N W}} \\
\theta_{i}^{G e_{N W}}=\chi\left(\frac{\theta_{i}^{G e_{M a c r}}}{\theta_{i}^{S i_{M a c r}}}\right)\left(\frac{1+b_{1}^{i} D}{b_{2}^{i}+b_{3}^{i} D}\right)^{S i_{N W}}
\end{array}
$$

De este modo, estamos haciendo una aproximación muy simple, pero que nos deja únicamente un parámetro de ajuste, $\chi$. Este parámetro lo vamos a obtener de las medidas de la conductividad térmica experimental para NWs lisos de Germanio procedentes de la referencia [20]. Los NWs medidos en dicha referencia están por debajo de los $25 \mathrm{~nm}$, y por tanto presentan confinamiento fonónico. En este rango nuestro modelo no era capaz de predecir el comportamiento lineal de a conductividad térmica con la temperatura; sin embargo, el valor obtenido a $300 \mathrm{~K}$ 


\section{TRANSPORTE TÉRMICO EN NWS SEMICONDUCTORES}

con nuestro modelo para la conductividad térmica de un NW de silicio de $22 \mathrm{~nm}$ si que es muy cercano al experimental. Asumiendo que lo mismo sucederá para un NW de germanio, se ha determinado el valor de $\chi$ para que la conductividad térmica calculada con nuestro modelo para un NW de $19 \mathrm{~nm}$ de germanio coincida con la determinada experimentalmente en [20], i.e $\chi=0,9$. Para confirmar la validez de estas hipótesis tan fuertes, se han calculado las conductividades térmicas de distintos NWs para los cuales existe una predicción teórica con otro formalismo completamente distinto [31]; con los resultados que se pueden observar en la figura 2.17 .

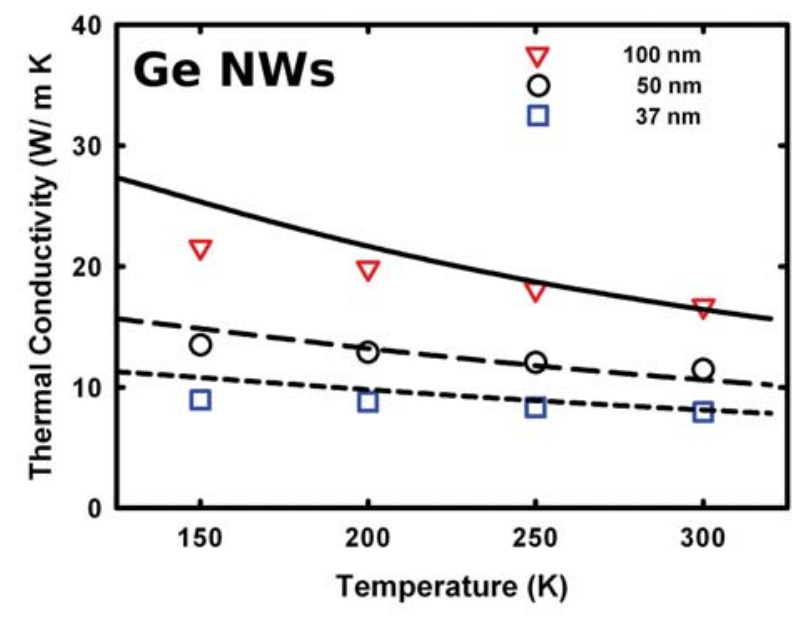

Figura 2.17: Conductividades térmicas predichas con el modelo de la referencia [31] (símbolos) y Conductividades térmicas predichas con nuestro modelo para cuatro NWs lisos de germanio de diferentes diámetros.

Puede observarse como las conductividades térmicas predichas mediante nuestro modelo se corresponden perfectamente en el rango de temperaturas de interés con las predichas por N. Mingo en [31] . Esto implica que bajo las hipótesis tomadas, nuestro modelo es al menos igual de fiable que el dado en [31] para predecir la conductividad térmica de NWs de Ge lisos, conservando además todas sus propiedades para aplicarlo a NWs rugosos.

Para aplicar el modelo construido para aleaciones en NWs de $S i_{1-X} G e_{X}$ se ha de determinar además el parámetro de Bowing característico de la aleación dado 
por $C_{\text {Bowing }}$. Dado que los NWs son estructuras cuasi-unidimensionales, cualquier in-homogeneidad de la aleación va a suponer un enorme centro de scattering que dificulta el transporte fonónico, y por tanto el efecto de la aleación debe de ser mucho más importante que para cristales volúmicos.

La determinación de este parámetro, así como la comprobación de la validez del modelo para predecir la conductividad térmica, requiere de una comparación con datos experimentales para NWs de $S i_{1-X} G e_{X}$. Existen diversos autores que han medido la conductividad térmica en NWs de $S i_{1-X} G e_{X}[23,24,75]$, pero la mayoría de ellos está compuesta por NWs muy gruesos, de entre $150 \mathrm{~nm}$ a $300 \mathrm{~nm}$, los cuales están fuera del rango de interés y validez de nuestro modelo ( $D \in$ $[\sim 25 \mathrm{~nm}, \sim 120 \mathrm{~nm}])[23,24]$. Por ello, los únicos válidos son los procedentes de la referencia [75]; aunque de los NWs de $S i_{1-X} G e_{X}$ medidos en [75] no todos son adecuados, ya que muchos son estructuras core-shell con una gruesa coraza de oxido que puede afectar al transporte térmico. Teniendo en cuenta esto, se han escogido los cuatro únicos NWs de $S i_{1-X} G e_{X}$ de la referencia [75] adecuados para este estudio. El valor del parámetro $C_{\text {Bowing }}$ se ha ajustado para un único dato, el NW de $45 \mathrm{~nm}$ y una composición con un $10 \%$ de germanio, pudiéndose observar en la figura 2.18 como para el resto de NWs se obtienen unos valores para la conductividad térmica compatibles con los datos experimentales. La buena correspondencia entre conductividades térmicas experimentales y teóricas para NWs de $S i_{1-X} G e_{X}$ nos indica que nuestro modelo es una buena herramienta de calculo para predecir la conductividad térmica de los NWs de $S i_{1-X} G e_{X}$. En la figura $2.19 \mathrm{~A}$ ) se ha representado la predicción de la conductividad térmica para un NW de $S i_{1-X} G e_{X}$ liso de $56 \mathrm{~nm}$ en función de la composición de la aleación. Puede observarse el buen acuerdo con los datos experimentales, y como la conductividad térmica de la aleación desciende abruptamente incluso para una pequeña proporción de una de las componentes de la aleación, tal y como se predecía en [58], y se mostraba experimentalmente en [24]. En la figura 2.19 B), se muestra la influencia del diámetro y la composición de la aleación en la conductividad térmica de los NWs. Puede comprobarse como el diámetro es relevante para aleaciones muy pobres en una de las componentes, mientras que cuando la proporción de la componente minoritaria aumenta, la conductividad térmica se hace casi independiente del diámetro, esto es 


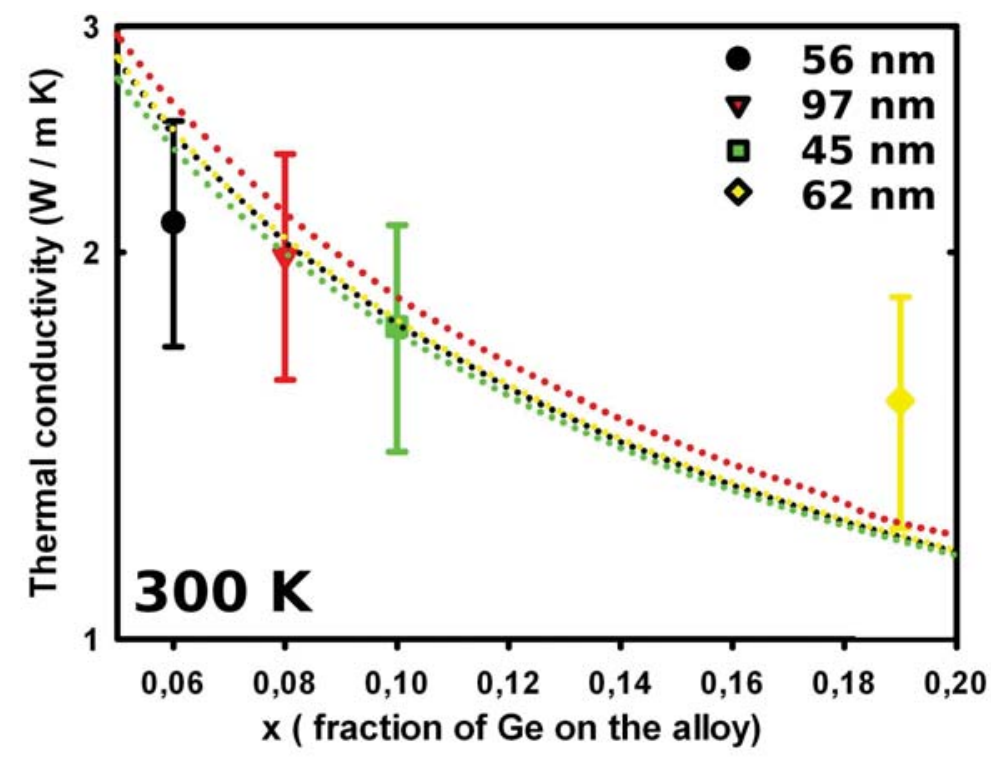

Figura 2.18: Conductividad térmica para 4 cuatro NWs lisos de $S i_{1-X} G e_{X}$ con diferentes composiciones y diámetros procedentes de la referencia[75] (símbolos) y conductividades térmicas calculadas con nuestro modelos para NWs de $S i_{1-X} G e_{X}$ con los mismos diámetros (lineas). Puede observarse el buen acuerdo con los datos experimentales en todos los casos (nótese la escala es logarítmica). 
debido al enorme peso de los eventos de scattering por la aleación en comparación con los otros factores de scattering.

Por último, podemos observar el efecto de la rugosidad en NWs de $S i_{1-X} G e_{X}$. Dado que todo el efecto de la rugosidad está englobado en el termino de scattering de superficie, las expresiones encontradas para $\zeta$ en el apartado anterior son validas para el modelo que predice la conductividad en una aleación. En la referencia [25] se mostraba experimentalmente como el efecto de la rugosidad se sumaba al de la aleación, provocando una reducción aún mayor de la conductividad térmica en NWs de $S i_{1-X} G e_{X}$ rugosos. Podemos ver confirmado este efecto en la figura 2.20, donde se ha calculado la conductividad térmica en función de la composición para un NW de $100 \mathrm{~nm}$ liso y otro rugosos. Puede observarse como la conductividad térmica se reduce por efecto de la rugosidad hasta valores cercanos a $0.7 \mathrm{~W} / \mathrm{mK}$ para una aleación al $50 \%$, lo que supone supone una conductividad térmica 15 veces menor que la de la aleación macroscopica equivalente y 215 veces menor que la del silicio volúmico. 

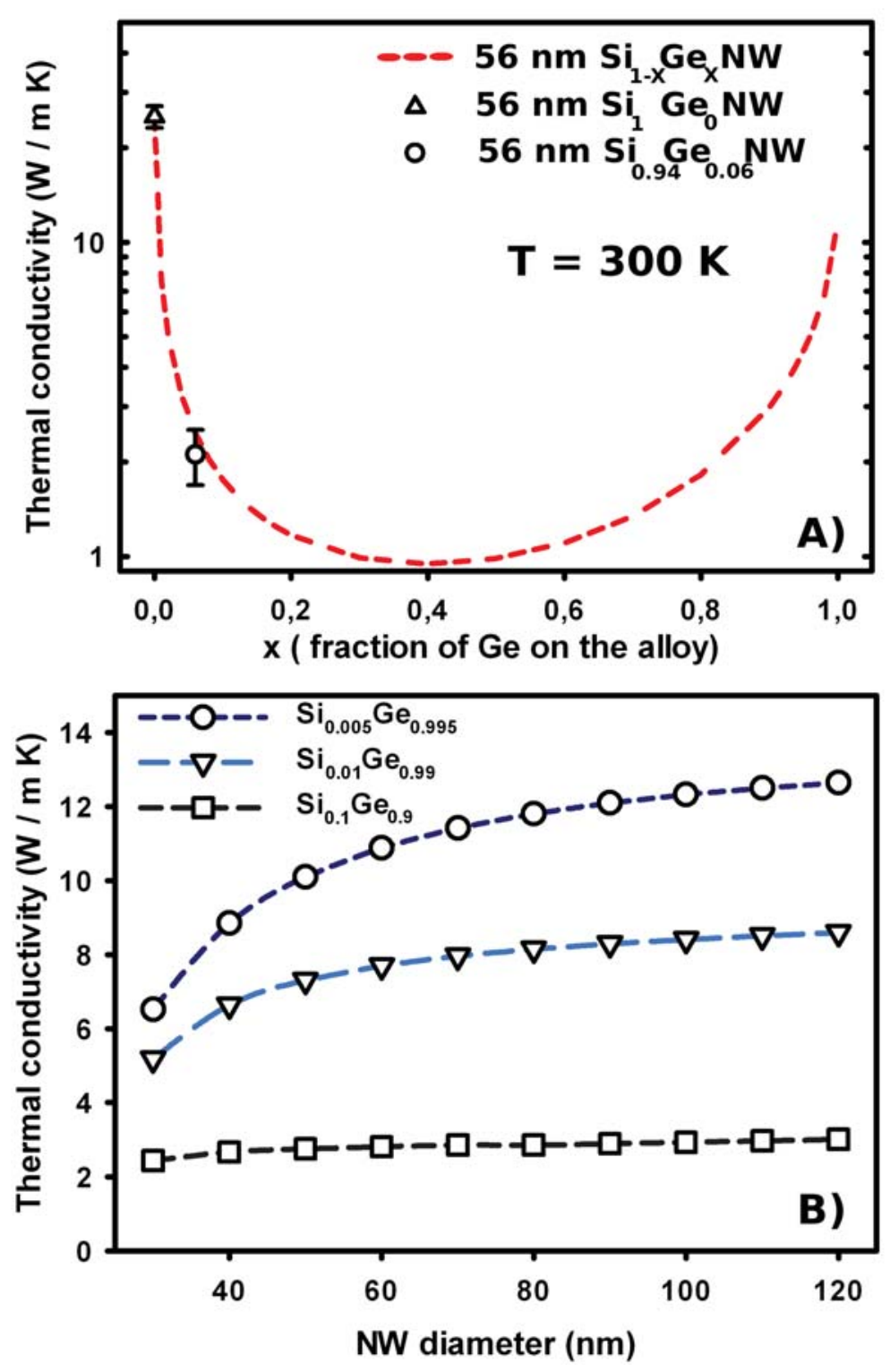

Figura 2.19: A) Conductividad térmica en función de la composición de la aleación para un NW liso de $S i_{1-X} G e_{X}$ de $56 \mathrm{~nm}$ de diámetro (linea), se muestran también las conductividades térmicas experimentales procedentes de [11] y [75], observándose un excelente ajuste a la curva predicha por nuestro modelo. B) Conductividad térmica de NWs de $S i_{1-X} G e_{X}$ en función del diámetro para tres composiciones diferentes. Puede observarse como la influencia del diámetro va disminuyendo según se enriquece la aleación en el componente minoritario 


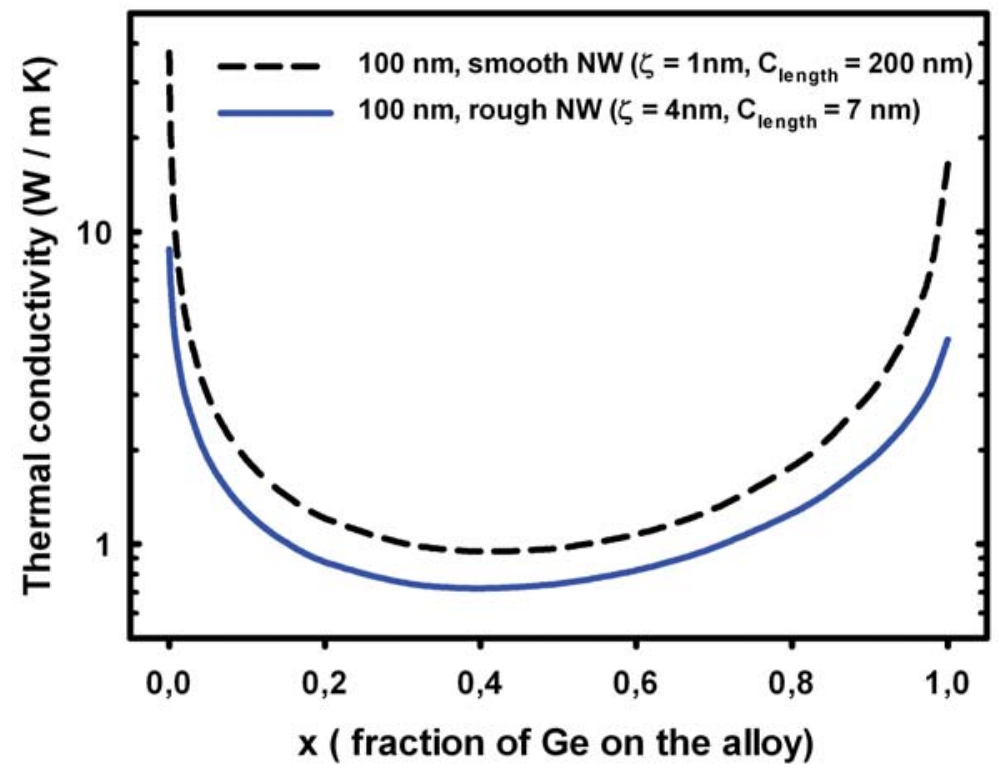

Figura 2.20: Conductividad térmica calculada en función de la composición para dos NW de $100 \mathrm{~nm}$, uno liso y otro rugoso. Puede observarse como el efecto de la rugosidad se superpone al de la aleación, obteniéndose una conductividad térmica muy baja 



\subsection{Bibliografía}

[1] Cui Y, Lieber C.M. Functional Nanoscale Electronic Devices Assembled using Silicon Nanowire Building Blocks. Science 2001;291, 851.

[2] Hochbaum A.I, Chen R, Delgado R.D, Liang W, Garnett E.C, Najarian M, Majumdar A, Yang P. Rough Silicon Nanowires as High Performance Thermoelectric Materials. Nature 2008;451, 163.

[3] Kayes B.M, Atwater H.A, Lewis N.S. Comparison of the device physics principles of planar and radial p-n junction nanorod solar cells. J.Appl.Phys. 2005;97, 114302.

[4] Xie X.Q, Liu W.F, Oh J.I, Shen W.Z. Optical absorption in c-Si/a-Si:H core/shell nanowire arrays for photovoltaic applications. Appl.Phys.Lett. 2011;99, 033107.

[5] Singh N, Buddharaju K.D, Manhas S.K, Agarwal A, Rustagi S.C, Lo G.Q, Balasubramanian N, Kwong D.L. Si, SiGe Nanowire Devices by Top-Down Technology and Their Applications. IEEE Trans.Electron Devices 2008;55, 3107.

[6] Westover T, Jones R, Huang J.Y, Wang G, Lai E, Talin A.A. Photoluminescence, Thermal Transport, and Breakdown in Joule-Heated GaN Nanowires. NanoLett. 2009;9, 257.

[7] Shi L, Yao D, Zhang G, Li B. Large thermoelectric figure of merit in Si1-xGex nanowires. Appl.Phys.Lett. 2010;96, 173108.

[8] Mingo N. Thermoelectric figure of merit of II-VI semiconductor nanowires. Appl.Phys.Lett. 2004;85, 5986.

[9] Mingo N. Thermoelectric figure of merit and maximum power factor in III-V semiconductor nanowires. Appl.Phys.Lett. 2004;84, 2652.

[10] Shi L, Li D, You C, Jang W, Kim D, Yao Z, Kim P, Majumdar A. Measuring Thermal and Thermoelectric Properties of One-Dimensional Nanostructures Using a Microfabricated Device. J.Heat Transfer 2003;125, 881.

[11] Li D, Wu Y, Kim P, Shi L, Yang P, Majumdar A. Thermal conductivity of individual silicon nanowires. Appl.Phys.Lett. 2003;83, 2934.

[12] Hippalgaonkar K, Huang B, Chen R, Sawyer K, Ercius P, Majumdar A. Fabrication of Microdevices with Integrated Nanowires for Investigating Low-Dimensional Phonon Transport. NanoLett. 2010;10 4341. 


\section{TRANSPORTE TÉRMICO EN NWS SEMICONDUCTORES}

[13] Li D.Y, Wu Y, Fan R, Yang P.D, Majumdar A. Thermal conductivity of Si/SiGe superlattice nanowires. Appl.Phys.Lett. 2003;83, 3186.

[14] Glassbrenner J.C, Slack G.A. Thermal Conductivity of Silicon and Germanium from $3^{\circ}$ K to the Melting Point. Phys.Rev. 1964;134, 4A 1058.

[15] Campbell I. H, Fauchet P.M. The effects of microcrystal size and shape on the one phonon Raman spectra of crystalline semiconductors. Solid St. Commun. 1986;58, 739.

[16] Zhou J, Jin C, Seol J.H, Li X, Shi L. Thermoelectric properties of individual electrodeposited bismuth telluride nanowires. Appl.Phys.Lett 2005;87, 133109.

[17] Guthy C, Nam C, Fischer J.E. Unusually low thermal conductivity of gallium nitride nanowires. J.Appl.Phys. 2008;103, 064319.

[18] You C. Large Thermoelectric Figure-of-Merits from SiGe Nanowires by Simultaneously Measuring Electrical and Thermal Transport Properties. NanoLett. 2012;12, 2918.

[19] Shi L, Hao Q, Choongho Y, Mingo N, Xiangyang K. Thermal conductivities of individual tin dioxide nanobelts. Appl.Phys.Lett. 2004;84, 2638.

[20] Wingert M.C, Chen Z.C.Y, Dechaumphai E, Moon J, Kim J, Xiang J, Chen R. Thermal Conductivity of Ge and Ge-Si Core-Shell Nanowires in the Phonon Confinement Regime. NanoLett 2011;11, 5507.

[21] Park Y, Kim J, Kim H, Kim I, Lee K, Seo D, Choi H, Kim W. Thermal conductivity of VLS-grown rough Si nanowires with various surface roughnesses and diameters. Appl.Phys. A 2011;104,7.

[22] Lim J, Hippalgaonkar K, Andrews S.C, Majumdar A, Yang P. Quantifying Surface Roughness Effects on Phonon Transport in Silicon Nanowires. NanoLett. 2012;12, 2475.

[23] Kim H, Kim I, Choi H, Kim W. Thermal conductivities of Si1-xGex nanowires with different germanium concentrations and diameters. Appl.Phys.Lett. 2010;96, 233106.

[24] Kim H, Park Y, Kim I, Kim J, Choi H, Kim W. Effect of surface roughness on thermal conductivity of VLS-grown rough Sil-xGex nanowires. Appl.Phys. A 2011;104, 23.

[25] Balandin A, Wang K.L. Significant decrease of the lattice thermal conductivity due to phonon confinement in a free-standing semiconductor quantum well. 
Phys.Rev B 1998;58, 1544.

[26] Parrott J. E., Stuckes A. D. Thermal Conductivity of Solids, New York: Methuen; 1975.

[27] Zou J, Balandin A. Phonon heat conduction in a semiconductor nanowire. J.Appl.Phys. 2001;89, 2932.

[28] Ziman J.M. Electrons and Phonons New York: Oxford University Press; 1967.

[29] Volz S.G, Chen G. Molecular dynamics simulation of thermal conductivity of silicon nanowires. Appl.Phys.Lett. 1999;75 2056.

[30] Mingo N. Calculation of Si nanowire thermal conductivity using complete phonon dispersion relations. Phys.Rev. B 2003;68, 113308.

[31] Mingo N, Yang L. Predicting the Thermal Conductivity of Si and Ge Nanowires. Nano Lett. 2003;3, 1713.

[32] Herring C. Role of Low-Energy Phonons in Thermal Conduction. Phys.Rev 1954;95, 954.

[33] Chen Y, Diyu L, Lukes J.R, Majumdar A. Monte Carlo Simulation of Silicon Nanowire Thermal Conductivity. Journal of Heat Transfer 2005;127, 1129

[34] Huang M, Chong W, Chang T. The lattice thermal conductivity of a semiconductor nanowire. J.Appl.Phys. 2006;99 114318.

[35] Liang L.H, Li B. Size-dependent thermal conductivity of nanoscale semiconducting systems. Phys.Rev. B 2006;73, 153303.

[36] Murphy P.G, Moore J.E. Coherent phonon scattering effects on thermal transport in thin semiconductor nanowires. Phys.Rev. B 2007;76, 155313.

[37] Tian Z, Esfarjani K, Shiomi J, Henry A.S, Chen G. On the importance of optical phonons to thermal conductivity in nanostructures. Appl.Phys.Lett 2011;99, 053122 .

[38] Ward A, Broido D.A. Intrinsic phonon relaxation times from first-principles studies of the thermal conductivities of Si and Ge. Phys.Rev B 2010;81, 085205.

[39] Kazan M, Pereira S, Coutinho J, Correia M.R, Masri P. Role of optical phonon in Ge thermal conductivity. Appl.Phys.Lett 2008;92, 211903.

[40] Kazan M, Guisbiers G, Pereira S, Correia M, Masri P, Bruyant A, Volz S, Royer P. Thermal conductivity of silicon bulk and nanowires: Effects of isotopic composition, phonon confinement, and surface roughness. J.Appl.Phys 2010;107, 083503. 


\section{TRANSPORTE TÉRMICO EN NWS SEMICONDUCTORES}

[41] Callaway J. Model for Lattice Thermal Conductivity at Low Temperatures. Phys.Rev. 1959;113, 1046.

[42] Holland M.G. Analysis of Lattice Thermal Conductivity. Phys.Rev 1963;132, 2461.

[43] Carruthers P. Theory of Thermal Conductivity of Solids at Low Temperatures. Rev.Mod.Phys 1961;33, 92.

[44] Srivastava G.P. The Physics of Phonons, Bristol:Adam Hilger; 1990.

[45] Alvarez F.X, Jou1 D. Size and frequency dependence of effective thermal conductivity in nanosystems. J.Appl.Phys, 2008;103, 094321.

[46] Guyer R.A, Krumhansl J.A. Solution of the Linearized Phonon Boltzmann Equation. Phys.Rev 1966;148, 766.

[47] Guyer R.A, Krumhansl J.A. Thermal Conductivity, Second Sound, and Phonon Hydrodynamic Phenomena in Nonmetallic Crystals. Phys.Rev 1966;148, 778. [48] Alvarez F.X., Jou D, Sellitto A. Phonon hydrodynamics and phonon-boundary scattering in nanosystems. J.Appl.Phys, 2009;105, 014317.

[49] Sellitto A. Alvarez F.X, Jou D. Second law of thermodynamics and phononboundary conditions in nanowires. J.Appl.Phys. 2010;107, 064302.

[50] Moore A.L, Saha S.K, Prasher R.S, Shi L. Phonon backscattering and thermal conductivity suppression in sawtooth nanowires. Appl.Phys.Lett 2008;93, 083112. [51] Sellito A, Alvarez F.X, Jou D. J.Appl.Phys, Temperature dependence of boundary conditions in phonon hydrodynamics of smooth and rough nanowires. 2010;107, 114312.

[52] Martin P, Aksamija Z, Pop E, Ravaioli U. Impact of Phonon-Surface Roughness Scattering on Thermal Conductivity of Thin Si Nanowires. Phys.Rev.Lett. 2009;102, 125503.

[53] Kosevich Y.A, Savin A.V. Reduction of phonon thermal conductivity in nanowires and nanoribbons with dynamically rough surfaces and edges, EPL 2009;88, 14002.

[54] Liu L,Chen X. Effect of surface roughness on thermal conductivity of silicon nanowires. J.Appl.Phys 2010;107, 033501.

[55] Donadio D, Galli G. Atomistic Simulations of Heat Transport in Silicon Nanowires. Phys.Rev.Lett. 2009;102, 195901. 
[56] Luisier M. Investigation of thermal transport degradation in rough Si nanowires. J.Appl.Phys 2011;110, 074510.

[57] Sadhu J, Sinha S. Room-temperature phonon boundary scattering below the Casimir limit. Phys.Rev. B 2011;84, 115450.

[58] Yang N, Zhang G, Li B. Ultralow Thermal Conductivity of Isotope-Doped Silicon Nanowires. NanoLett. 2008;8, 276.

[59] Chen J, Zhang G, Li B. Tunable thermal conductivity of Sil -x Gex nanowires. Appl.Phys.Lett 2009;95, 073117.

[60] Wang Z, Mingo N. Diameter dependence of SiGe nanowire thermal conductivity. Appl.Phys.Lett 2010;97, 101903.

[61] Morelli D.T., Heremans J.P, Slack G.A. Estimation of the isotope effect on the lattice thermal conductivity of group IV and group III-V semiconductors. Phys.Rev B 202;66, 195304.

[62] Peierls R. E. Quantum Theory of Solids New York: Oxford University Press; 1955.

[63] Sharma P.C, Frank Rose M. Three-phonon scattering processes and their role in phonon thermal conductivity of silicon. Journal Solid State Chemistry 1998;73, 92.

[64] Slack G. A. Thermal Conductivity of CaF2, MnF2, CoF2, and ZnF2 Crystals. Phys.Rev. 1961;122, 1451.

[65] Klemens P.G. Solid State Physics, Vol 7, p.1 New York: Academic; 1958.

[66] Asen-Palmer M. Bartkowski K, Gmelin E, Carona M, Zhernov A.P, Inyushkin V.A, Taldenkov A, Ozhogin V.I, Itoh K.M, Haller E.E. Thermal conductivity of germanium crystals with different isotopic compositions. Phys.Rev. B 1997;56, 9431.

[67] Heron J. S, Fournier T, Mingo N, Bourgeois O. Mesoscopic Size Effects on the Thermal Conductance of Silicon Nanowire. NanoLett. 2009;9, 1861.

[68] Soffer S.B. Statistical Model for the Size Effect in Electrical Conduction. J.Appl.Phys. 1967;38, 1710.

[69] Kronrod, A. S. Nodes and Weights of Quadrature Formulas - transl. from Consultans Bureau, New York 1965.

[70] Omar M.S, Taha H.T. Lattice dislocation in Si nanowires. Physica B 2009;404, 5203 


\section{TRANSPORTE TÉRMICO EN NWS SEMICONDUCTORES}

[71] Goodnick S. M, Ferry D. K, Wilmsem C. W, Liliental Z, Fathy D, Krivanek O. L. Surface Roughness at the Si(100)-SiO2 Interface, Phys. Rev. B. 1985;32, 8171.

[72] Rasigni G, Rasigni M, Palmari J, Dussert C, Varnier F. Statistical Paramenters for Random and Pseudorandom Rough Surfaces, J. Opt. Soc. Am. A, 1988;5, 99.

[73] Maycock P. D, Thermal Conductivity of Silicon, Germanium, III-V Compounds and III-V Alloys, Solid State Electronics, 1967;10, 161.

[74] Miyazaki S, Takahashi H, Yamashita H, Narasaki M, Hirose M. Cristalline Solids, 2002;299, 148.

[75] Lee E.K, Yin L. Lee y, Lee J. W, Lee S. J, Lee J, Cha S. N, Wang D, Hwang G. S, Hippalgaonkar K, Majumdar A, Yu C, Choi B. L, Kim J. M, Kim K. Large Thermoelectric Figure-of-Merits from SiGe Nanowires by Simultaneously Measuring Electrical and Thermal Transport Properties., Nano Lett. 2012;12, 1918. 
He who works with the door open gets all kinds of interruptions, but he also occasionally gets clues as to what the world is and what might be important. de NWs semiconductores bajo un haz láser

\subsection{Introducción}

Los NWs semiconductores han atraído una gran atención entre la comunidad científica dado su potencial como base sobre la que construir los dispositivos electrónicos y optoelectrónicos del futuro [ $1-5]$. Como ya hemos observado en el capítulo anterior, muchas propiedades físicas de estas estructuras son fuertemente dependientes de su tamaño, siendo especialmente destacable cuando su diámetro es tan pequeño como para que aparezcan efectos de confinamiento cuántico [6, 7]. Sin llegar tener que llegar a este límite, hemos visto en el capítulo anterior como la dimensión del diámetro de los NWs de silicio y $S i_{1-X} G e_{X}$ afecta al transporte térmico [8-11]. La reducida conductividad térmica de estas estructuras semiconductoras nanométricas 


\section{ESTUDIO TEÓRICO DE LA DISTRIBUCIÓN DE TEMPERATURA Y SU EFECTO EN LA SEÑAL RAMAN DE NWS SEMICONDUCTORES BAJO UN HAZ LÁSER}

en comparación con sus homólogos volúmicos, va a ser determinante en el comportamiento de dispositivos basados en estos NWs que operan en condiciones de alta densidad de potencia [12].

Para entender mejor el comportamiento de estas estructuras, son necesarias técnicas de caracterización capaces de ofrecer información a escala submicrométrica. Dentro de estas técnicas de caracterización, las basadas en haces láser como son la PL (fotoluminescencia) [13 - 15], absorción óptica [16 - 18], espectroscopia Raman [19 - 27], o PC (fotocorriente) [27, 28], son comúnmente utilizadas para la caracterización de nanoestructuras semiconductoras.

El análisis de los resultados obtenidos por métodos basados en haces láser debe de ser tratado con mucho cuidado cuando se trabaja en nanoescala; debido a que la muestra es menor que el haz láser y menor que la longitud de onda de los fotones del haz, la interacción láser-nanoestructura no es igual que en escala macroscopica. Si no se tiene en cuenta esto, los resultados obtenidos pueden interpretarse de forma incompleta o incluso errónea. La caracterización óptica en NWs semiconductores puede realizarse sobre conjuntos de NWs o sobre NWs individuales. Dado el reducido volumen de material iluminado en un solo NW por el haz láser, su caracterización óptica puede resultar complicada por lo débil de la señal obtenida, teniendo que alcanzar un compromiso entre la potencia del láser necesaria para su detección y el calor inducido por el láser en el NW, el cual puede distorsionar la interpretación de los datos obtenidos. El uso de conjuntos de NWs garantiza que la señal obtenida es más intensa con menor potencia, y por tanto facilita su análisis. Sin embargo, cuando se utilizan madejas de NWs la respuesta óptica proviene de diferentes NWs que están siendo simultáneamente excitados por el haz láser. Estos NWs pueden ser diferentes entre sí en cuestión de tamaños o morfología superficial, y por tanto su contribución a la señal muy inhomogénea, introduciendo por tanto una distorsión en la información recibida; e.g. la temperatura alcanzada por los NWs debida al calor inducido por el haz láser dificulta la interpretación de los datos recolectados $[12,19,21-24,29$ - 32], ya que ciertas propiedades ópticas presentan una fuerte dependencia con la temperatura [12, $33-37]$.

En la investigación llevada a cabo en este trabajo, se ha estudiado la caracterización mediante espectroscopia Raman de NWs de silicio y $S i_{1-X} G e_{X}$. La correcta interpretación de los espectros obtenidos experimentalmente mediante esta técnica 
va a estar estar sujeta a una completa comprensión de la interacción láser-NW. Para ello, en este capítulo se recopilan los resultados del estudio teórico llevado a cabo sobre como es esta interacción láser-NW y como puede afectar a la señal Raman.

\subsection{Modelado de los efectos térmicos debidos a la in- teracción láser-NW}

Para modelar la interacción láser-NW, es necesario estimar cual es la cantidad de energía absorbida por el NW cuando está bajo el haz láser. A diferencia de la situación macroscopica para la caracterización óptica mediante técnicas láser, en las que el tamaño del área iluminado por el haz láser , o spot del haz láser, es mucho más pequeño que la muestra analizada, en el análisis de NWs, el tamaño de ese spot es cerca de un orden de magnitud mayor que el diámetro de los NWs. El láser utilizado en espectroscopia micro-Raman atraviesa un pinhole de tamaño menor que su longitud de onda, por lo que presenta una distribución intensidad no homogénea en el spot, dada por una distribución de Airy (Figura 3.1 A):

$$
I(r)=I_{0}^{A i r y}\left(\frac{2 J_{1}\left(\frac{\pi}{\lambda N} r\right)}{\left.\frac{\pi}{\lambda N} r\right)}\right)^{2}
$$

Siendo $\lambda$ la longitud de onda, $N$ la apertura numérica y $J_{1}$ la función de Bessel de primera especie. Esta distribución de intensidad puede aproximarse bastante bien por una distribución gaussiana dada por:

$$
I(r) \simeq I_{0} e^{-2\left(\frac{r^{2}}{w^{2}}\right)}
$$

Donde $w$ da cuenta del tamaño del área iluminada y se corresponde al punto en que la intensidad decae hasta $1 / e$ veces $I_{0}$ (figura $3.1 \mathrm{~B}$ ) ). Cuando la muestra sobre la que se efectúa la caracterización es macroscopica, esta distribución no homogénea de la intensidad del spot del láser pasa desapercibida, pero cuando el tamaño de la muestra es menor que el tamaño del spot, la intensidad que ilumina la muestra va a depender enormemente de la posición que ocupa el NW en el área iluminada por el láser. Esto va a ser muy relevante cuando la muestra se compone de varios NWs, puesto que cada uno va a estar iluminado con una intensidad dependiente de 


\section{ESTUDIO TEÓRICO DE LA DISTRIBUCIÓN DE TEMPERATURA Y SU EFECTO EN LA SEÑAL RAMAN DE NWS SEMICONDUCTORES BAJO UN HAZ LÁSER}

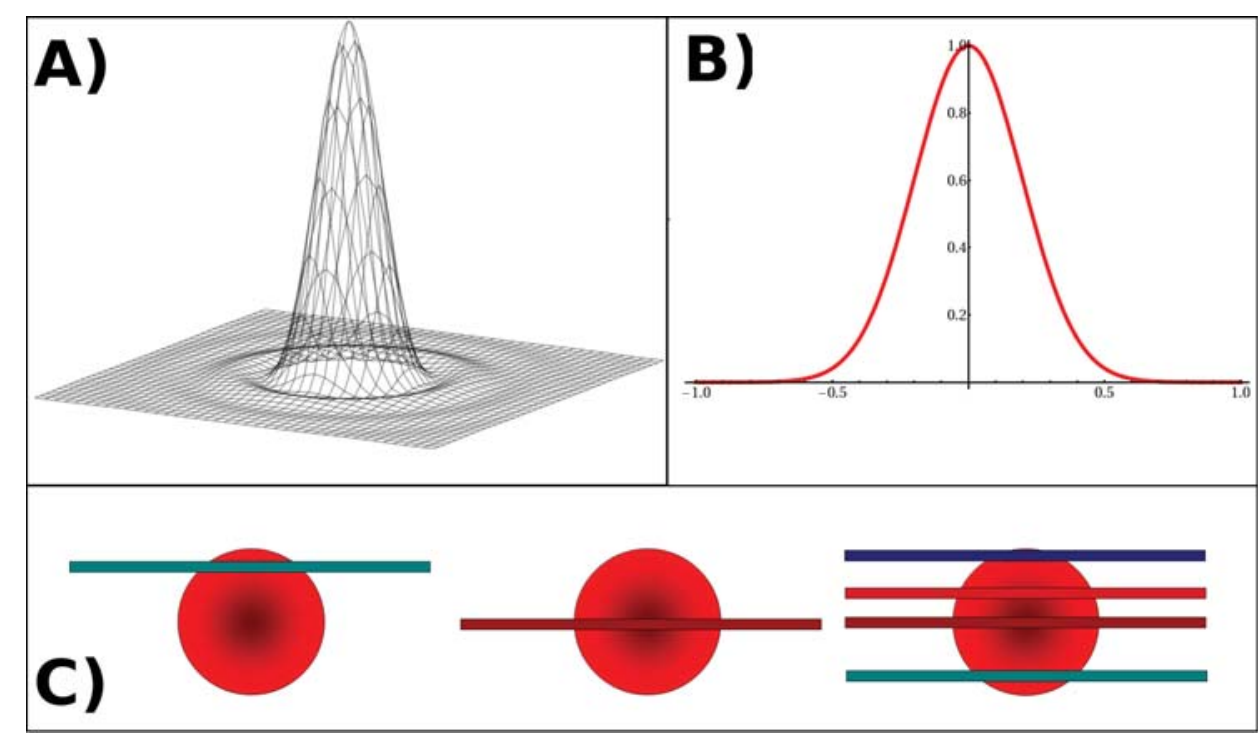

Figura 3.1: A) Representación tridimensional de la distribución de Airy para la intensidad de un haz láser (Fuente: Wikimedia Commons). B) Representación 2D de la distribución gaussiana equivalente para la intensidad de un haz láser. C) Representación de como son iluminados diferentes NWs de $100 \mathrm{~nm}$ dentro de un spot láser de 1 $\mu \mathrm{m}$ de diámetro.

su posición en el spot, y por tanto, incluso NWs idénticos tendrán una contribución diferente a la señal final (Figura 3.1 C) ).

Por otro lado, en espectroscopia microRaman las longitudes de onda típicamente utilizadas van desde el ultravioleta cercano $(\lambda \sim 300 \mathrm{~nm})$ a longitudes de onda superiores a los $1000 \mathrm{~nm}$. Por tanto, el diámetro de los NWs iluminados también es mucho más pequeño que la longitud de onda de los fotones que conforman el haz láser. Esto tiene un importante efecto en la absorción de la energía que portan los fotones del haz por el NW, siendo esta absorción muy dependiente del diámetro de los NWs [18, 35, 38, 39].

En un material volúmico, la energía absorbida por la muestra iluminada puede obtenerse a través del coeficiente de absorción, $\alpha$, mediante una ley de absorción exponencial del tipo Beer-Lambert, que nos dice como decae la intensidad del haz láser cuando atraviesa la muestra:

$$
I=I_{0} e^{-\alpha d}
$$


Donde $d$ es el espesor de la muestra. La energía en la muestra estará determinada por la cantidad de área de la muestra iluminada y la energía absorbida en profundidad caracterizada por esta ley exponencial. Si la iluminación no es homogénea, como en el caso de un láser focalizado de perfil gaussiano, la energía absorbida en la muestra iluminada debe ser modificada de acuerdo a esta distribución, resultando en una densidad de energía dependiente de las tres coordenadas espaciales en la muestra. En el caso de los NWs, la obtención de la densidad de energía en el NW iluminado no es tan simple, pero puede abordarse de forma equivalente a través del coeficiente de absorción $\alpha$. Dado que el diámetro del NW es mucho menor que la longitud de onda del haz láser, la superficie efectiva de absorción no coincide con la superficie geométrica del NW. Este efecto ha sido calculado y demostrado mediante la resolución de las ecuaciones de Maxwell en la aproximación de Mie para la interacción electromagnética láser-NW por diversos autores $[17,35,38]$. La forma de cuantificar este efecto es a través de la eficiencia de absorción, $Q_{a}$, definida como el cociente entre la sección eficaz de absorción y la sección geométrica del NW dada por el diámetro del mismo. El valor de $Q_{a}$ es muy dependiente de la longitud de onda del láser y sobre todo del diámetro de los NWs, pudiendo tomar para NWs de un mismo material valores próximos a cero, (i.e. la sección eficaz de absorción es mucho menor que la sección geométrica), o valores mayores a uno (i.e. la sección eficaz de absorción es mayor que la sección geométrica del NW). Podemos observar el efecto de $Q_{a}$ en la figura 3.2 A) para una muestra rectangular de dimensión lateral menor a la longitud de onda del láser. Cuando $Q_{a}$ es igual a uno, la sección de absorción coincide con la sección geométrica, tal y como ocurre para muestras macroscópicas. Cuando $Q_{a}$ es menor a uno, la sección de absorción es menor que la sección geométrica, lo que corresponde a una situación equivalente a tener una muestra de dimensión lateral más pequeña y por tanto una menor energía absorbida; concretamente, será la energía absorbida por una muestra de tamaño lateral igual al de la sección eficaz de absorción. Por tanto la densidad de energía en el NW es menor que la que le correspondería si se aplicase directamente el formalismo de la escala macroscópica. En el caso de tener $Q_{a}$ mayor a uno, el comportamiento es el inverso al anterior. El valor de $Q_{a}$ en simetría cilíndrica para estructuras de longitud infinita fue calculado por M. Kerker [42], pudiéndose extender este resultado para estructuras cuya longitud es mucho mayor que su 


\section{ESTUDIO TEÓRICO DE LA DISTRIBUCIÓN DE TEMPERATURA Y SU EFECTO EN LA SEÑAL RAMAN DE NWS SEMICONDUCTORES BAJO UN HAZ LÁSER}

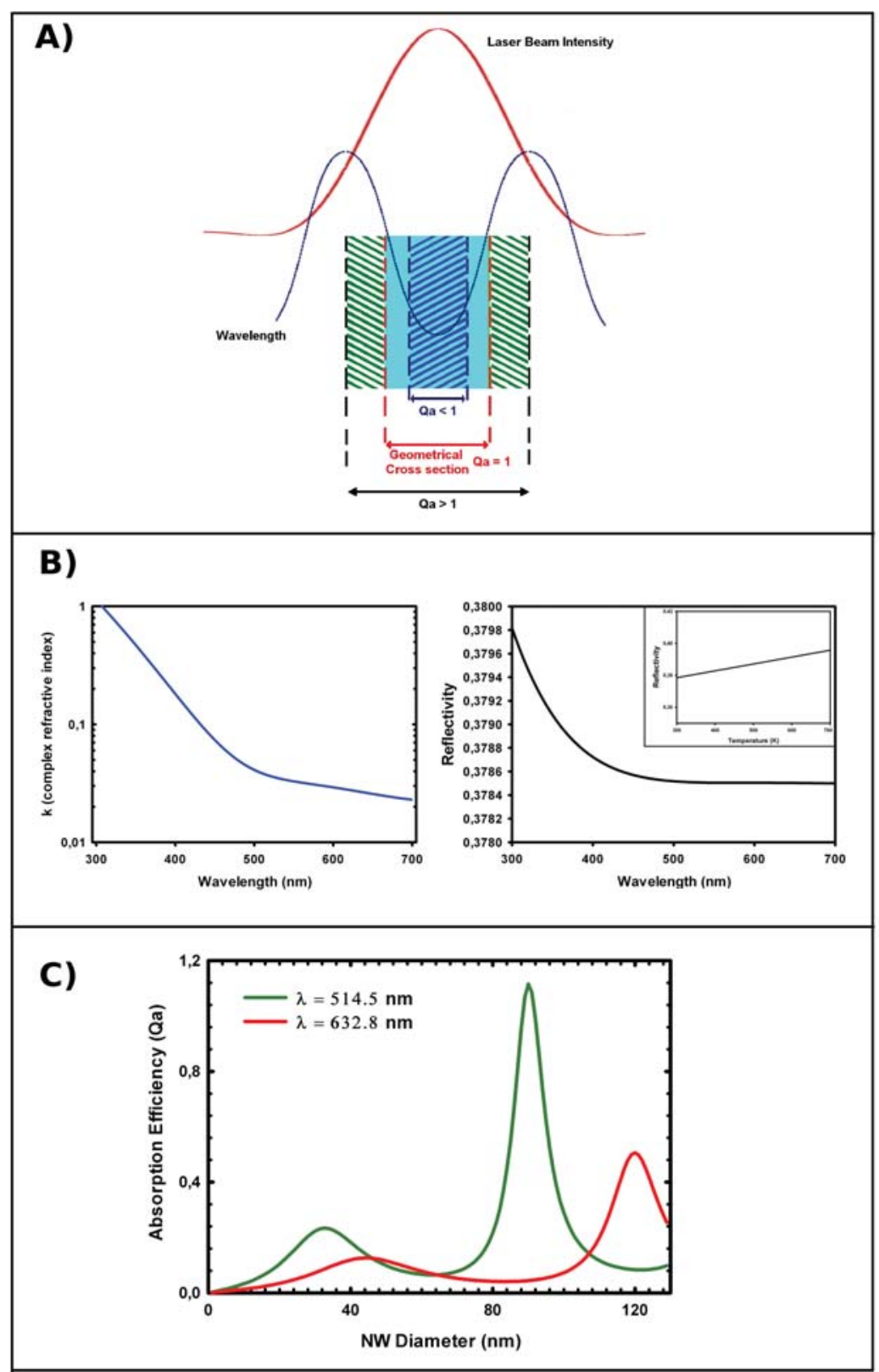

Figura 3.2: A) Esquema de como afecta el valor de $Q_{a}$ a la absorción de energía en una muestra con una dimensión lateral menor a la longitud de onda del haz láser incidente. B) Índice de refracción complejo para el silicio en función de la longitud de onda necesario para el cálculo de $Q_{a}$, a su lado Reflectividad calculada para el silicio en función de la longitud de onda y la temperatura. C) Cálculo de $Q_{a}$ para NWs de silicio en función del diámetro y para dos longitudes de onda típicas en espectroscopia Raman. Puede observarse como para algunos diámetros se producen resonancias en las que $Q_{a}$ es mayor que 1 . 
diámetro, como son los NWs, sin perdida de generalidad [36, 38, 42]. Dentro de esta aproximación, el valor de $Q_{a}$ se puede obtener como:

$$
\begin{array}{r}
\left.Q_{a}=\frac{2}{\xi}\left(R e\left(B_{0}+\sum_{n=1}^{\infty} B_{n}\right)-\left|B_{0}\right|^{2}-2 \sum_{n=1}^{\infty}\left|B_{n}\right|^{2}\right)\right) \\
B_{n}=\frac{k J_{n}(\xi) J_{n}^{\prime}(k \xi)-J_{n}^{\prime}(\xi) J_{n}(k \xi)}{k H_{n}(\xi) J_{n}^{\prime}(k \xi)-H_{n}^{\prime}(\xi) J_{n}(k \xi)}
\end{array}
$$

Donde $\xi=\frac{\pi D_{N W}}{\lambda}, J_{n}$ es la función de Bessel de primer orden, $H_{n}$ la función de Hankel de segundo orden y $k$ el índice de refracción complejo del material.

Utilizando los valores para el silicio volúmico (ver figura 3.2 B) ) se obtiene el valor de $Q_{a}$ para los NWs de silicio que están representados para dos longitudes de onda típicas en espectroscopia Raman en la figura 3.2 C). Podemos observar como $Q_{a}$ es muy dependiente del diámetro, tomando valores muy por debajo de la unidad en casi todo el rango de diámetros representado. Esto implica que salvo excepciones, los NWs de silicio absorben mucha menos energía que la que les correspondería por su sección geométrica. Podemos observar también como aparecen unos picos muy marcados para determinados diámetros en los que $Q_{a}$ puede ser mayor incluso a la unidad. Este resultado tan diferente de lo acontecido a nivel macroscópico conlleva a que la cantidad de energía absorbida por los NWs no dependa directamente de la sección geométrica de la muestra (i.e. del diámetro del NW) como ocurre en macroescala, sino a que un determinado NW absorba una mayor cantidad energía del haz láser que otro NW con un mayor diámetro bajo las mismas condiciones de iluminación. Por otro lado, es necesario considerar que no toda la energía del haz es susceptible de ser absorbida por la muestra, existe un parte que es directamente reflejada por superficie y que está representada a través de la reflectividad de la muestra. Para el silicio esté valor es próximo al $40 \%$ para las longitudes de onda estudiadas, tal y como puede observarse en la figura 3.2 B).

Otro factor fundamental para entender la interacción láser-NW es la conductividad térmica de los NWs, fundamentalmente porque es indispensable para resolver la ecuación del calor. En los NWs iluminados por un haz láser se está introduciendo una energía y si el NW no la disipa eficientemente se va a inducir un incremento de temperatura en el NW. Dado que la energía se absorbe en una parte muy locali- 


\section{ESTUDIO TEÓRICO DE LA DISTRIBUCIÓN DE TEMPERATURA Y SU EFECTO EN LA SEÑAL RAMAN DE NWS SEMICONDUCTORES BAJO UN HAZ LÁSER}

zada y con una distribución gaussiana a lo largo del NW, la conductividad térmica va a determinar como se distribuye esa energía a lo largo de todo el hilo. Como ya hemos visto en el capitulo anterior, la conductividad térmica de los NWs semiconductores es mucho más baja que sus homólogos volúmicos, siendo además muy dependiente del diámetro $[8,10,11,36,43,45]$. Por otro lado, sabemos que si la superficie de los NWs es muy rugosa la conductividad térmica puede descender a valores cercanos al límite del silicio amorfo, el cual se comporta como un buen aislante térmico $[9,44,46,47]$. El valor de la conductividad térmica va a ser muy relevante si los NWs están anclados a un substrato que actúa a modo de sumidero térmico,ó simplemente si la conductividad térmica es muy baja, ya que la energía depositada por el haz láser no va a poder ser distribuida por el NW y se acumulará en la zona iluminada. Cuantificar este efecto va a ser fundamental para una correcta interpretación de los espectros Raman obtenidos en este tipo de sistemas. Gracias al modelo construido para predecir la conductividad térmica en el capítulo 2 y en la referencia [48], podemos conocer la conductividad térmica para cualquier NW de silicio (ver figura 3.3) y por tanto resolver la ecuación del calor para el sistema láser-NW y analizar el efecto de la conductividad térmica en la distribución de temperatura dentro de los NWs.

\subsubsection{Modelo FEM para el trasporte térmico de un NW bajo un} haz láser

El calentamiento inducido en un NW de silicio por un láser que lo ilumina va a depender de la energía que este absorbe del haz, de su conductividad térmica y de las condiciones de disipación dadas por el medio que rodea al NW o a la existencia de sumideros térmicos. Como ya hemos podido observar, estas condiciones son bastante diferentes a las que se presentan para materiales en escala macroscópica, y por tanto es necesario realizar un estudio térmico de lo que sucede en la interacción láser-NW.

Para realizar este estudio se ha recurrido a un modelo de elementos finitos, utilizando para ello el software comercial COMSoL Multiphysics, y más concretamente 


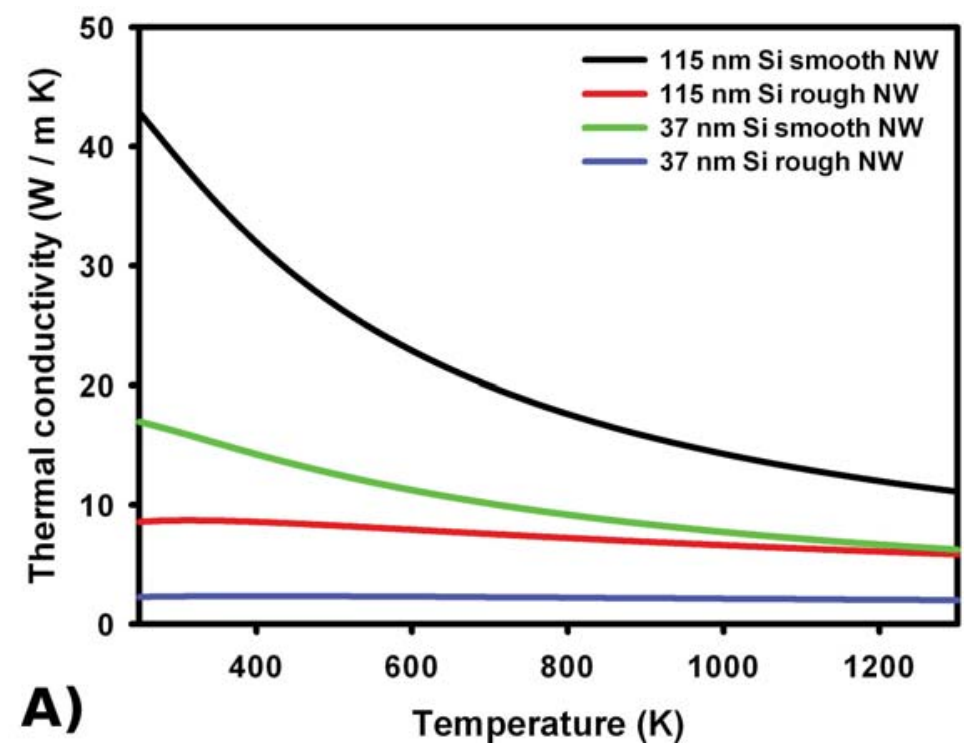

Figura 3.3: Conductividades térmicas simuladas con el modelo construido en el capítulo 2 para dos NWs de silicio de 115 y $37 \mathrm{~nm}$, tanto con superficies lisas como rugosas.

el modulo de transferencia de calor de este potente software de simulación de excelente reputación en el mundo científico.

Mediante este software, se ha generado un modelo 3D de las diferentes geometrías analizadas y se ha resuelto la ecuación del calor dada por:

$$
\nabla \cdot(-\kappa \nabla T)=Q
$$

Donde $\kappa$ es la conductividad térmica del NW analizado en cada caso y $Q$ la densidad de potencia/energía en cada punto del espacio. El estudio se ha realizado bajo condiciones estacionarias, para ello se asume que las medidas se realizan iluminando la muestra un tiempo suficiente para que el sistema a nanoescala alcance el equilibrio térmico y con un haz láser cuya intensidad es constante en el tiempo. Esta hipótesis se ha tomado después de realizar un estudio transitorio del tiempo necesario para que un NW alcance el equilibrio térmico y comprobar que este tiempo es varios ordenes de magnitud menor al segundo, que es la escala de tiempo común para realizar experimentalmente una medida Raman.

Para calcular el término $Q$, se tiene que tener en cuenta la potencia total que trans- 


\section{ESTUDIO TEÓRICO DE LA DISTRIBUCIÓN DE TEMPERATURA Y SU EFECTO EN LA SEÑAL RAMAN DE NWS SEMICONDUCTORES BAJO UN HAZ LÁSER}

porta el haz, y corregirla adecuadamente con los términos dados por la reflectividad, la absorción y la eficiencia de absorción, además de tener en cuenta la distribución gaussiana de la intensidad del haz.

La densidad de potencia introducida por el láser tiene una distribución gaussiana como la descrita en la ecuación 3.2, donde el parámetro que determina el máximo de la distribución gaussianas determina a través de la medida experimental de la potencia que deposita en la superficie focalizada por cada láser utilizado. Esta potencia se ha medido con un potenciometro NEW PORT $842 \mathrm{PE}, \mathrm{y} 1 / e$ veces este valor experimentalmente determinado debe ser igual a la potencia total calculada de integrar la ecuación 3.2 en un área dado por el tamaño del spot (que precisamente es la distancia radial del máximo para el que la potencia decae a $1 / e$ veces su valor máximo), este valor para el área efectivo de iluminación es algo menor a 1 $\mu \mathrm{m}$, y se estima en $\sim 0,9 \mu \mathrm{m}$.

La densidad de potencia en cada punto del interior del NW se obtiene de la ecuación 3.3, que a través del coeficiente de absorción $\alpha$ para la longitud de onda del haz láser, determina que cantidad de toda la potencia que transporta el haz en la posición del spot en la que se sitúa el NW es absorbida por el NW (recordemos que la potencia que ilumina el NW no es homogénea, sino que tiene una distribución espacial gaussiana dada por 3.2). Aquí debe tenerse en cuenta también el efecto de la reflectividad del NW, y que por tanto la potencia efectiva es 1 - Reflectividad veces la experimental. Finalmente esta densidad de potencia tiene que ser corregida por el efecto que introduce la eficiencia de absorción para cada NW, para ello hay que determinar relación entre la potencia absorbida por un NW con una sección eficaz dada por las dimensiones geométricas y la potencia absorbida por una estructura con un volumen corregido teniendo en cuenta la reducción el la dimensión lateral dada por $Q_{a}$ (ver figura 3.2).

La estructura tridimensional del NW ha sido modelada mediante la aplicación de diseño asistido por ordenador (CAD) que incorpora el propio software de COMSOL. Las geometrías generadas van desde la situación más simple de un cilindro, nanométrico en diámetro y micrométrico en longitud, completamente sumergido en un fluido (e.g. Aire, agua), a otras más complejas como las mostradas en la figura 3.4. En todas las situaciones simuladas se ha tomado la decisión de no considerar un substrato bajo la muestra. El efecto que introduce un substrato bajo la muestra 


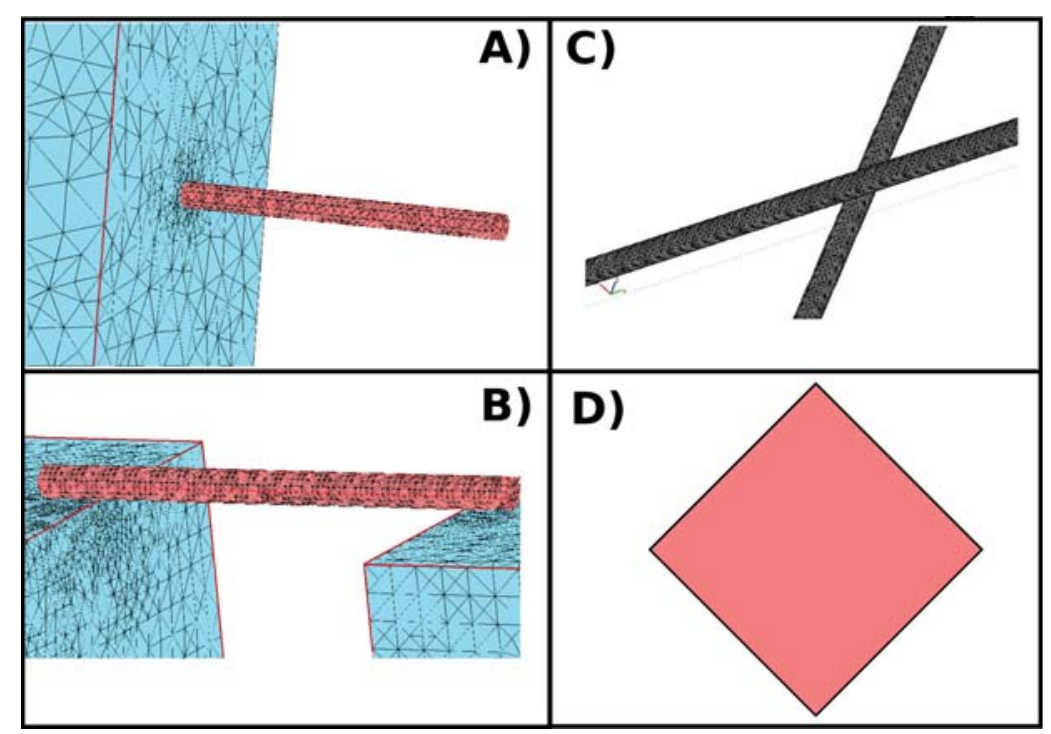

Figura 3.4: A) Geometría generada para un NW anclado por uno de sus extremos a un substrato. B) Geometría generada para un NW suspendido entre dos contactos térmicos perfectos. C) Geometría generada para dos NWs que se cruzan en el espacio. D) Aproximación tomada para las superficies de intercambio térmico con el medio que rodea al NW.

va a depender de como refleje este substrato el haz incidente; si se comporta como un espejo perfecto, el haz que atraviesa el NW, debilitado tras su paso por el NW, se reflejará y volverá a atravesar el NW, depositando de nuevo una parte de su energía; sin embargo, si el substrato es trasparente a la longitud de onda del haz incidente no existirá contribución alguna del haz reflejado. En ambos casos la diferencia estriba en la magnitud de la cantidad de energía depositada en el NW; es decir, su efecto es equivalente a tener un haz láser más potente. Por otro lado el substrato actuará como un contacto térmico, y dependiendo de lo bueno o malo que sea este contacto la energía confinada en el interior del NW será mayor o menor, situación equivalente a tener un haz láser menos energético. Por tanto, el efecto del substrato puede ser englobando en el estudio del comportamiento de la temperatura inducida en un NW en función de la potencia utilizada en el láser. Esto implica que aunque el substrato afecte a la magnitud de la temperatura, el comportamiento de la temperatura inducida en el NW por efecto de la escala y las características intrínsecas de los NWs puede considerarse independiente del substrato. 


\section{ESTUDIO TEÓRICO DE LA DISTRIBUCIÓN DE TEMPERATURA Y SU EFECTO EN LA SEÑAL RAMAN DE NWS SEMICONDUCTORES BAJO UN HAZ LÁSER}

Para resolver la ecuación 3.5 en la geometría generada es necesario establecer las condiciones de contorno adecuadas para cada situación simulada. En las geometrías mostradas en la figura 3.4 puede observarse que existen dos situaciones muy diferentes para estas condiciones de contorno. Cuando tomamos la situación en la que el NW está sumergido completamente en un fluido, las condiciones de contorno deben responder al intercambio de energía entre las superficies del NW y el fluido circundante. Típicamente, en las muestras experimentales el fluido que rodea al NW va a ser el aire del propio laboratorio, pero excepcionalmente también puede depositarse una gota de agua sobre la muestra para comprobar el efecto del medio en la señal recogida.

El intercambio de energía entre las paredes del NW y el aire puede modelarse a través de una expresión que tenga en cuenta el efecto convectivo del fluido y también la pérdida de energía en el NW por radiación. El efecto convectivo entre las superficies del NW y el fluido circundante requiere de un complejo estudio dinámico de la interacción fluido-superficie. Afortunadamente, para determinadas geometrías y fluidos como el aire y el agua, puede simplificarse mediante su descripción en la forma de un coeficiente de transmisión térmica, $h$ [40]. Para poder aplicar esto, se ha explotado una potente característica brindada por el modulo de transmisión térmica de COMSOL que permite aproximar las paredes curvadas del NW por superficies rectas de igual superficie, tal y como aparece en la figura 3.4 C). Bajo esta aproximación, la condición de contorno para las superficies del NW pueden describirse como una expresión de tipo Neumann dada por:

$$
-\mathbf{n}(-\kappa \nabla T)=h\left(T_{0}-T\right)+\epsilon \sigma\left(T_{0}^{4}-T^{4}\right)
$$

Donde $\epsilon$ y $\sigma$ son la emisividad del material y la constante de Stefan-Boltzmann respectivamente, $T_{0}$ la temperatura del medio de inmersión y $h$ el coeficiente de transmisión térmica del fluido. La ecuación 3.6 es en realidad la forma típica de describir el transporte térmico entre dos superficies por conducción y radiación, pero a través de una modificación del parámetro $h$, esta ecuación describe bien el intercambio de energía con un fluido como el aire y del agua cuando este está lejos 
de su punto de ebullición [40]. El valor de $h$ puede obtenerse como :

$$
h=C^{\prime}\left(\frac{\triangle T}{l}\right)^{n} \kappa_{\text {fluido }}\left(\frac{\beta g \rho^{2} C_{p}}{\mu \kappa_{\text {fluido }}}\right)^{2}
$$

Donde $\kappa_{\text {fluido }}$ es la conductividad térmica del fluido, $C_{p}$ su capacidad calorífica a presión constante, $\beta$ el coeficiente de dilatación volumétrica del fluido, $\mu$ el coeficiente de viscosidad dinámica, $\rho$ la densidad, $l$ la dimensión de la superficie (ver figura $3.4 \mathrm{C}$ ) ). Por ultimo $C^{\prime}$ y $n$ son dos coeficientes dependientes de la geometría y de la dimensión de la muestra, cuyo valor puede encontrarse en [40] y que COMSOL determina automáticamente a través de la geometría creada con el módulo de CAD incorporado en el propio COMSOL. El valor de $h$ depende de la temperatura a través de la dependencia con la temperatura de las magnitudes físicas que lo conforman. Esta dependencia está implementada en el modulo de transferencia térmica de COMSOL a través de una compleja ecuación derivable de ajuste a los datos experimentales de la base de datos incorporada en COMSOL para cada material.

Por último, las geometrías que incorporan substratos requieren una condiciones de contorno diferentes. En los puntos en los que el NW se une al substrato (e.g. figura 3.4 A), unión entre la base del NW y el substrato) se toman condiciones de contorno de continuidad. Mientras que las condiciones de contorno para los substratos están tomadas en base a que estos substratos son muy grandes en comparación con los NWs; por tanto, pueden considerarse infinitos y la condición de contorno para sus fronteras son condiciones de sumidero térmico perfecto, i.e de tipo Dirichlet.

Por último, la obtención de un buen resultado cuando se utiliza como herramienta de cálculo los elementos finitos depende del mallado y del método escogido (ver capítulo 1). Para determinar la combinación óptima entre número de elementos del mallado y orden del método, se ha ido aumentando el número de elementos y el orden de los elementos lagrangianos hasta que las soluciones obtenidas dejaban de ser sensibles estos factores.

Una vez configurada nuestra plataforma de simulación en el modulo de transporte térmico de COMSOL, se han estudiado de forma separada los diferentes factores que intervienen en el calentamiento inducido en un NW iluminado por un haz láser. Gracias a este análisis, se pueden desacoplar las contribuciones de cada factor a la 


\section{ESTUDIO TEÓRICO DE LA DISTRIBUCIÓN DE TEMPERATURA Y SU EFECTO EN LA SEÑAL RAMAN DE NWS SEMICONDUCTORES BAJO UN HAZ LÁSER}

temperatura inducida en un NW, y por tanto se va a poder interpretar mejor qué sucede cuando experimentalmente se analizan los NWs semiconductores mediante técnicas de caracterización basadas en haces láser.

\subsubsection{Influencia de la potencia, longitud de onda y medio de in- mersión en la temperatura alcanzada en un NW de silicio ilumi- nado por un haz láser}

Como punto de partida se ha tomado un NW de silicio liso de $5 \mu \mathrm{m}$ de $37 \mathrm{~nm}$ y diámetro. La elección de estas características geométricas no responde a ningún interés especial más allá del de corresponder a unas dimensiones típicas en los NWs reales [8]. Mediante el modelo implementado en $C O M S O L$, se ha calculado la temperatura inducida para este NW de silicio en función de la potencia del haz láser incidente, de la longitud de onda del mismo y del medio de disipación que rodea al NW. En este caso, el NW se considera completamente inmerso en el fluido y que por tanto solo puede transmitir la energía de su interior al medio que lo rodea. El resultado de estas simulaciones puede verse en la figura 3.5, donde se representa la temperatura máxima alcanzada en el NW cuando el láser incide en el centro del NW y además el NW está centrado en el spot gaussiano.

El rango de potencias seleccionado es el rango típico de operación en técnicas de caracterización Raman, PL o PC para los láseres con las longitudes de onda analizadas. Puede observarse la enorme dependencia de la temperatura inducida con la longitud de onda utilizada; este efecto proviene de dos factores acoplados en nanoescala, la diferencia entre los coeficientes de absorción del silicio para cada longitud de onda $\left(3,8974 \times 10^{5} \mathrm{~m}^{-1}\right.$ para $\lambda=632,8 \mathrm{~nm}$ vs $1,4977 \times 10^{6} \mathrm{~m}^{-1}$ para $\lambda=514,5 \mathrm{~nm}[50]$ ) y los diferentes valores de $Q_{a}$ en función de la longitud de onda para este diámetro $\left(Q_{a}^{514,5} \simeq 0,22\right.$ vs $\left.Q_{a}^{632,8} \simeq 0,10\right)$.

El papel jugado por el medio de disipación puede observarse al comparar entre las figuras $3.5 \mathrm{~A}$ ) y $3.5 \mathrm{~B}$ ), donde están representadas las temperaturas alcanzadas por el NW de prueba cuando éste está sumergido en aire o en agua y absorbe la misma potencia del haz con independencia del medio. Cabe destacar que debido a la especial geometría que caracteriza a los NWs, la relación entre la superficie exterior y el volumen encerrado es muy grande. Debido a esta propiedad, los NWs han 
sido propuestos por algunos autores como eficientes estructuras para la disipación térmica [51]. Sin embargo, cuando observamos la figura 3.5 A) podemos observar que el NW de silicio inmerso en aire se calienta incluso para muy bajas potencias, lo que indica que no comunica de forma eficiente la energía al medio y no se comporta como una buena estructura disipadora. Mientras que cuando el medio es agua, el NW prácticamente no se calienta, transmitiendo de una forma muy eficiente la temperatura de su interior al medio que lo rodea.

Este resultado puede parecer contradictorio, pero en realidad no es tal. En escala macroscopica la relación entre la superficie y el volumen es un buen indicativo de si una estructura es eficiente a la hora de disipar el calor de su interior, tanto si el fluido con el que está en contacto es aire o agua; sin embargo, en nanoescala, cuando el fluido es un gas, y por tanto su densidad es muy baja, la cantidad de partículas del gas que tocan la superficie del NW con las que intercambiar energía es muy escasa. Por otro lado, en un líquido, que es mucho más denso, la cantidad de moléculas en contacto con la superficie con las que intercambiar energía es mucho mayor, aproximándose más al comportamiento en escala macroscopica, y por tanto obteniendo el comportamiento de una estructura que es muy buena disipando energía, tal y como se observa en la figura 3.5 B). De este modo, es la cantidad de superficie y no su relación con el volumen interno la que hace que el intercambio energético sea muy ineficiente en fluidos poco densos como los gases. En nuestro modelo, este comportamiento está incluido en la dependencia de parámetro $h$ con la densidad en la ecuación 3.7 que caracteriza el intercambio de energía y que depende cuadráticamente de la densidad del fluido, y que por tanto es cerca de 6 ordenes de magnitud mayor para el agua que para el aire.

Este marcado efecto de la temperatura alcanzada por un NW por influencia de un haz láser en función del medio de disipación ha sido comprobado experimentalmente por varios autores en $[19,24]$.

\subsubsection{Influencia de las dimensiones geométricas en la temperatu- ra inducida por un haz láser en un NW de silicio}

Las dimensiones de los NWs juegan un papel fundamental en la temperatura inducida en el NW por efecto del haz láser, siendo especialmente relevante el papel 


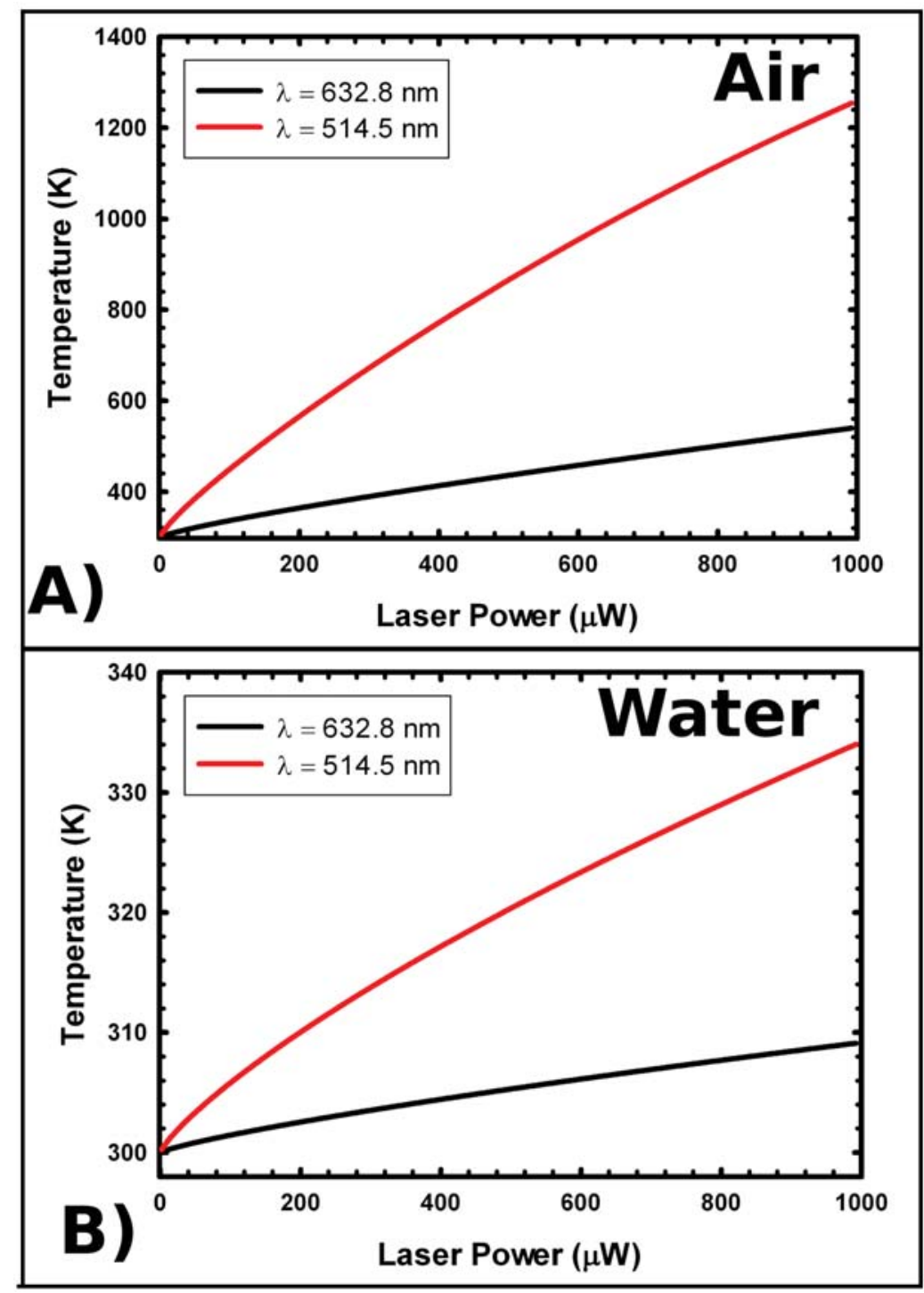

Figura 3.5: A) Temperatura alcanzada por un NW liso de silicio de $5 \mu \mathrm{m}$ de longitud y $37 \mathrm{~nm}$ de diámetro completamente inmerso en aire en función de la potencia del haz láser incidente. La linea roja representa la temperatura alcanzada cuando el haz láser tiene una longitud de onda de $514.5 \mathrm{~nm}$ (láser verde), mientras que la curva negra muestra la temperatura en el NW cuando el haz tiene una longitud de onda de 632.8 nm (láser rojo). A) figura de idéntica descripción a la anterior, pero en este caso el NW está inmerso en agua en vez de en aire. 
jugado por el diámetro de los NWs; no olvidemos que tanto la cantidad de energía absorbida como su transporte dependen de magnitudes fuertemente dependientes del diámetro del NW.

Dada la distribución gaussiana de la potencia del haz láser, y que la longitud de los NWs típicamente es mayor que el tamaño del spot láser, la conductividad térmica determina el equilibrio termodinámico entre la energía absorbida del haz láser y como se distribuye en el NW. Por otro lado, en nanoescala la cantidad de energía absorbida por el NW no es directamente dependiente del diámetro del NW, sino que está modulada por el valor de $Q_{a}$ que determina el volumen efectivo de absorción. Teniendo en cuenta estos efectos, en las figuras 3.6 y 3.7 se muestra como afectan las dimensiones del NW a la temperatura que en él se induce por efecto del haz láser, situándose en ambos casos el haz láser centrado en el punto medio del NW.

En la figura 3.6 se muestra la temperatura alcanzada por un NW de silicio liso con $5 \mu \mathrm{m}$ de longitud cuando un láser de potencia variable, y con una longitud de onda de $632.8 \mathrm{~nm}$ se focaliza en su punto medio. Dado que la disipación de energía por el aire que rodea al NW es muy mala, desde un punto de vista macroscópico, cabría esperar que los NWs más gruesos alcanzasen temperaturas más altas al acumular mucha más energía en su interior que los NWs más finos; sin embargo puede observarse claramente el efecto introducido por la eficiencia de absorción $Q_{a}$, la cual modula la energía absorbida del haz para cada diámetro, y causa que un NW de $40 \mathrm{~nm}$ se caliente tanto como un NW el doble de grueso con tan solo la mitad de potencia incidente. Puede observarse también como dependiendo del diámetro del NW iluminado se inducen temperaturas de varias decenas de grados incluso para valores de potencia considerados como muy bajos $(P \sim 10 \mu W)$ en técnicas de caracterización micro-Raman, micro-PL o micro-PC; por tanto, cuando uno realiza un experimento de caracterización en el que interviene un haz láser, se debe probar de algún modo que la muestra analizada no está siendo calentada con la potencia utilizada, ya que lo que para algunos NWs puede considerarse una potencia baja, para otros es suficiente como para elevar considerablemente su temperatura.

Por otro lado, en la figura 3.7 puede observarse como evoluciona la temperatura en el interior de un NW iluminado por un haz láser de $200 \mu \mathrm{W}$ y $514.5 \mathrm{~nm}$ de longitud de onda, en función de cualquier combinación de longitudes y diámetros. Para un 


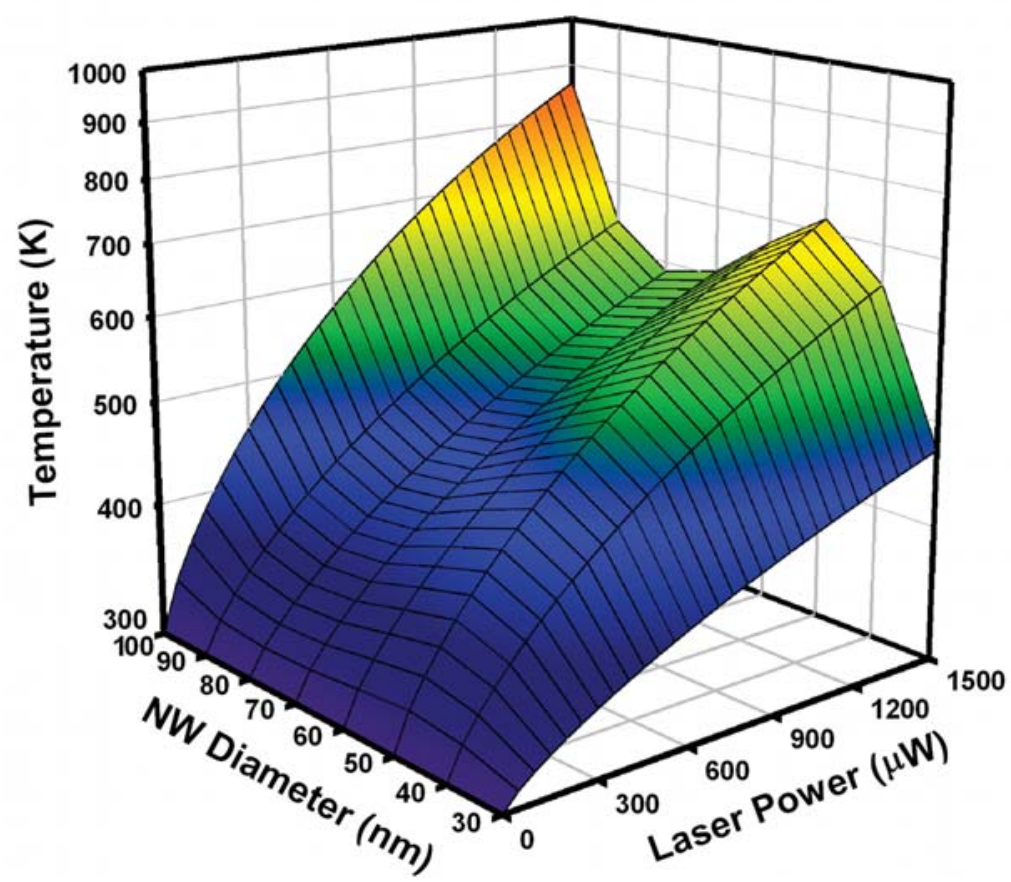

Figura 3.6: Representación tridimensional de la temperatura alcanzada por un NW de silicio de $5 \mu \mathrm{m}$ de longitud, completamente rodeado por aire, en función del diámetro del NW y de la potencia del láser incidente, el cual tiene una longitud de onda de 632.8 $\mathrm{nm}$ 
mismo diámetro se observa como la temperatura decrece cuanto más largo es el NW, resultado esperable dado que cuanto más largo es el NW mayor volumen de material en el que repartir la energía localmente absorbida del haz láser y también mayor superficie de disipación. Puede observarse que pese a la baja conductividad térmica de los NWs de silicio en comparación con el silicio volúmico, la distribución de energía es bastante eficiente, causando que la diferencia de temperatura alcanzada entre los NWs más cortos y los más largos sea muy diferente. Por tanto de nuevo observamos como la geometría de los NWs va a ser determinante cuando se analice la señal obtenida de ellos mediante un haz láser, ya que incluso para NWs de idéntico diámetro, la temperatura alcanzada por ellos bajo las mismas condiciones de iluminación puede diferir en cientos de grados Kelvin si sus longitudes son diferentes.

\subsubsection{Influencia de la conductividad térmica en la temperatura alcanzada por un NW rugoso de silicio iluminado por un haz láser}

Sabemos que la conductividad térmica de los NWs semiconductores es mucho menor que la de estructuras volúmicas del mismo material; sin embargo, hemos comprobado como los NWs de silicio de superficie lisa tienen un buen comportamiento en lo que concierne al transporte térmico (Fig 3.7). Cuando tratamos con NWs rugosos, la conductividad térmica puede verse reducida hasta un orden de magnitud por debajo de la que presentan los NWs equivalentes de superficie lisa [2], por lo que este efecto puede ser muy relevante en la temperatura alcanzada por los NWs iluminados por un haz láser. En este apartado se va a tratar cual es precisamente la influencia de la conductividad térmica en distintos escenarios.

Una configuración muy común cuando se trabaja con NWs es aquella en la que el NW está anclado al substrato en el que ha sido crecido, o aquel en el que el NW está anclado por sus extremos conectando dos substratos (e.g. puente térmico, dispositivo eléctrico basado en NW con contactos óhmicos, etc...). Dado que el intercambio energético con el aire que rodea al NW es muy deficiente, en este tipo de configuraciones la disipación está dominada por el transporte de la energía desde el foco térmico local al substrato que actúa como sumidero térmico. La calidad de 


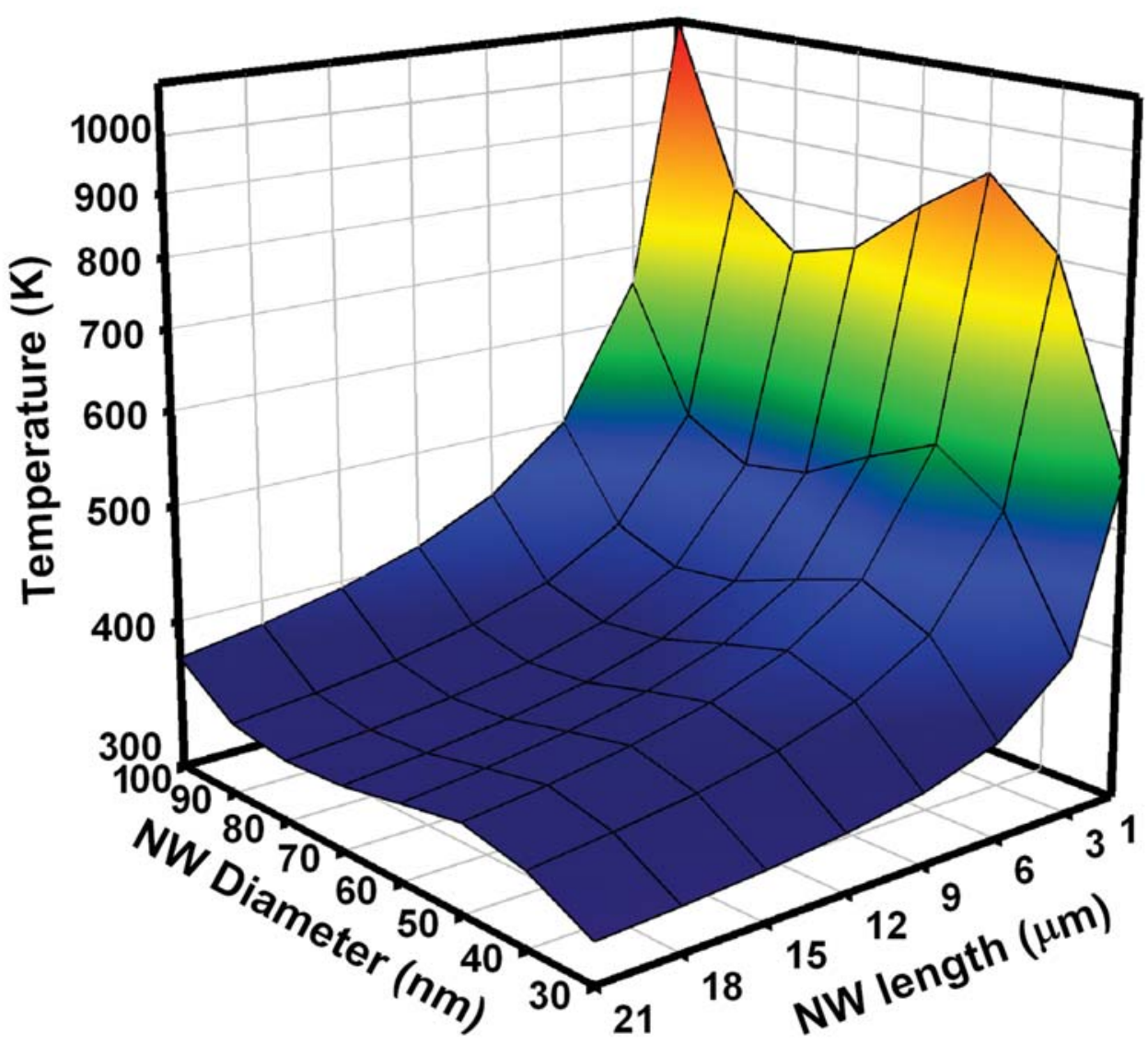

Figura 3.7: Representación tridimensional de la temperatura alcanzada por un NW de silicio liso en función del diámetro y longitud del NW cuando este es iluminado por una haz láser de $200 \mu \mathrm{W}$ y $514.5 \mathrm{~nm}$ de longitud de onda. 
este sumidero térmico es un problema aparte, ya que si no se garantiza un contacto de resistencia térmica muy baja (idealmente nula) se recupera una situación equivalente a la de un NW completamente rodeado por aire.

La relevancia de la conductividad térmica en una configuración con sumideros térmicos se muestra en la figura 3.8. En ella se ha simulado la temperatura máxima alcanzada por un NW de $37 \mathrm{~nm}$ de silicio, el cual se encuentra anclado a un substrato infinito de silicio a una temperatura de $300 \mathrm{~K}$ (ver figura $3.4 \mathrm{~A}$ ) ) mediante un contacto de resistencia térmica nula. La temperatura se representa en función de la conductividad térmica del NW y de su longitud cuando un haz láser de 514.5 $\mathrm{nm}$ de longitud de onda y $200 \mu \mathrm{W}$ incide en el punto medio del NW en cada caso. La conductividad térmica está calculada para un NW de $37 \mathrm{~nm}$ mediante el modelo construido en el capitulo 2, variándose los parámetros característicos de la rugosidad de la superficie para obtener una reducción en pasos del $10 \%$ en la conductividad térmica a $300 \mathrm{~K}$. Cuanto más corto es el NW más baja es la temperatura alcanzada por el NW, justo al revés que cuando no existe el sumidero térmico (ver Figura 3.7 para comparar), esto se debe a que cuanto más cerca está el substrato de la fuente térmica, mejor es la transferencia de la energía al sumidero térmico. Cuanto más baja es la conductividad térmica más se penaliza el transporte hacia el sumidero térmico, y por tanto más alta es la temperatura del NW. El efecto de la conductividad térmica es más evidente cuanto mayor es la distancia del foco térmico al substrato, ya que la energía tiene que recorrer una mayor distancia hasta llegar al substrato, y la disminución de la conductividad térmica dificulta mucho ese transporte de energía. Este efecto es especialmente evidente cuando el NW es muy largo, ya que cuando la conductividad térmica es muy baja el efecto del sumidero térmico prácticamente desaparece. Estas simulaciones se han realizado asumiendo que el contacto con el substrato es perfecto, esto implica que el efecto del sumidero térmico es muy relevante, obteniéndose unas temperaturas muy bajas en comparación con la situación en la que el NW está completamente rodeado por aire sin sumidero térmico; sin embargo, las medidas experimentales en este tipo de configuraciones con NWs anclados al substrato muestran que los NWs alcanzan unas temperaturas mucho mayores cuando son iluminados por potencias similares a las aquí utilizadas [24], lo que nos indica que la unión con el substrato dista mucho de tener una resistencia térmica nula. La existencia de esta resistencia térmica 


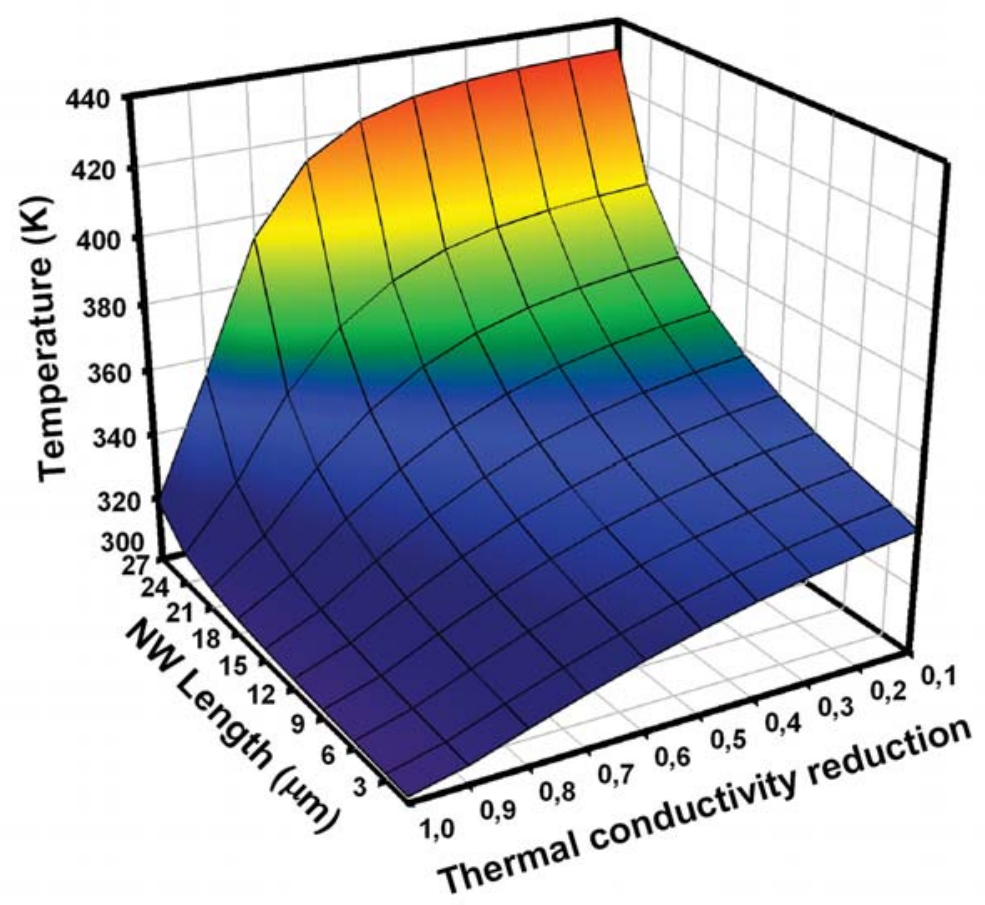

Figura 3.8: Representación tridimensional de la temperatura alcanzada por un NW de silicio rugoso en función de la conductividad térmica y de su longitud cuando el NW está anclado a un sumidero térmico (substrato) por uno de sus extremos (ver figura 3.4 A) ). El modelo se ha resulto para una configuración dada por un NW de $37 \mathrm{~nm}$ de diámetro, iluminado por un haz láser de $200 \mu \mathrm{W}$, con una longitud de onda de 514.5 nm y centrado en el punto medio del NW 
en la unión entre el substrato y el NW está relacionada con la presencia de una zona densamente poblada de defectos y dislocaciones de la red en la zona de unión entre el NW en el substrato, esta zona actúa como una barrera para el transito de los fonones que transportan la energía del NW al substrato cristalino [49].

Otro punto fundamental en el que la conductividad térmica del NW juega un papel determinante, es el que atañe a la distribución de temperatura dentro del NW cuando este es iluminado por un haz láser. Como ya hemos podido observar, en los NWs iluminados por haces láser con potencias del orden de las utilizadas en las técnicas de caracterización micro-Raman, micro-PL, etc.., pueden inducirse temperaturas muy elevadas. La señal procedente de la zona iluminada es sensible a la temperatura, por tanto es necesario distinguir entre los gradientes de temperatura existentes en toda la longitud del NW y los gradientes de temperatura en el interior de la zona directamente iluminada, para los cuales algunos autores han establecido unos valores de que superan los $600 \mathrm{~K} / \mu \mathrm{m}$ [22]. Sin embargo, la existencia de unos gradientes tan enormes en el interior de un NW únicamente está basada en la forma anómala que toma el espectro Raman adquirido sobre conjuntos de NWs [2].

La distribución de temperatura en el interior de los NWs se ha analizado mediante una simulación en el modelo FEM implementado en $C O M S O L$, teniendo en cuenta para ello las diferentes conductividades térmicas que un NW de un determinado diámetro puede tener dependiendo de las características de su superficie. El resultado puede verse para dos configuraciones diferentes en las figuras 3.9 A) y 3.9 B), perteneciendo la primera a un NW completamente sumergido en aire y la segunda a un NW con sus dos extremos unidos a un substrato mediante una unión de resistividad térmica nula. En ambos casos se observa como cuando la conductividad térmica se reduce, la temperatura máxima aumenta, esto se debe a que el transporte a lo largo de la estructura se dificulta al disminuir la conductividad térmica, y por tanto existe una mayor energía acumulada en un menor volumen de material. Un resultado muy relevante es el concerniente a los gradientes de temperatura en la zona iluminada por el láser, mostrando que para ambas configuraciones dicho gradiente no supera la decena de grados incluso para la configuración con menor conductividad térmica, correspondiente a un NW muy rugoso, y por tanto no son justificables unos gradientes de varios cientos de grados $\mathrm{K} / \mu \mathrm{m}$ como los supuestos 


\section{ESTUDIO TEÓRICO DE LA DISTRIBUCIÓN DE TEMPERATURA Y SU EFECTO EN LA SEÑAL RAMAN DE NWS SEMICONDUCTORES BAJO UN HAZ LÁSER}

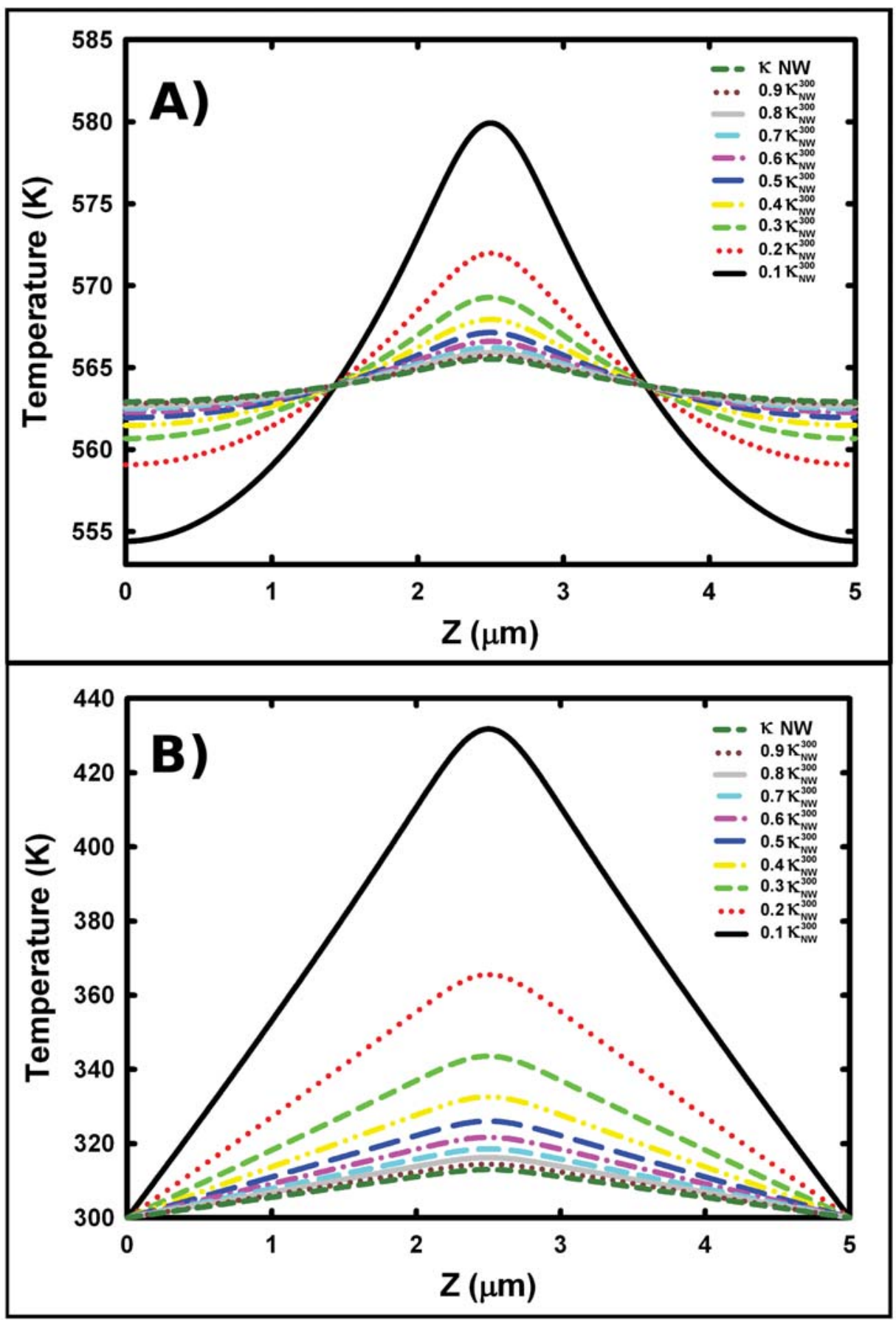

Figura 3.9: A) Distribución de temperatura a lo largo de un NW de silicio con un diámetro de $37 \mathrm{~nm}$ y $5 \mu \mathrm{m}$ de longitud para distintas conductividades térmicas calculadas mediante la ecuación 2.17. La potencia del láser es de $200 \mu \mathrm{W}$ con una longitud de onda de $514.5 \mathrm{~nm}$ y está centrado en el punto medio del NW. B) Distribución de temperatura en un NW idéntico al descrito en A) pero con sus dos extremos conectados a un substrato infinito de silicio. En esté caso la potencia es $500 \mu \mathrm{W}$ en vez de los $200 \mu \mathrm{W}$ utilizados en $\mathrm{A}$ ) 
en [22]. La existencia de unos gradientes de temperatura tan grandes en el volumen iluminado solo pueden suceder si la conductividad térmica tiene un valor tan bajo que prácticamente suprima el transporte térmico a lo largo de la estructura, confinando toda la energía depositada por el haz en la zona iluminada. Confinar tanta energía en tan poco espacio requiere de unos valores para la conductividad térmica menores a $0.05 \mathrm{~W} / \mathrm{mK}$, que es más de un orden de magnitud por debajo del valor mínimo reportado para NWs de aleaciones semiconductoras muy rugosas, por lo que parece muy poco probable que esto pueda suceder en NWs de silicio.

Por último, una geometría similar a la generada para la simulación representada en al figura 3.9 B) puede utilizarse para validar el modelo comparándolo con resultados experimentales. Para ello se recurre a una estructura como la que puede observarse en 3.4 B), donde un NW de silicio se sitúa con sus extremos apoyados sobre dos substratos que hacen de sumideros térmicos perfectos, mientras que el resto del NW está suspendido entre ambos substratos y por tanto, completamente rodeado por aire. En un dispositivo de este estilo se sabe experimentalmente que la temperatura inducida por un haz láser enfocado en puntos a lo largo de la parte suspendida del NW sigue una distribución parabólica dada por [33, 34, 37]:

$$
\triangle T(Z)=-\frac{P}{\kappa A}\left(\frac{(Z)^{2}}{L}-\frac{(Z) L}{4}\right)
$$

Siendo $P$ la potencia absorbida por el NW, $L$ la longitud de la parte suspendida, $A$ la sección del NW, $\kappa$ la conductividad térmica y $Z$ la posición del NW en la que está enfocado el haz láser. Dado que podemos extraer todos estos datos del modelo, podemos representar estas parábolas para diferentes conductividades térmicas en un NW con un diámetro y longitud determinado. El resultado puede observarse en la figura 3.10, donde se ha representado la temperatura obtenida del modelo y se ha representado en función de la posición en la que se focaliza el NW a lo largo de la parte suspendida del NW. Se ha representado también las parábolas predichas para cada conductividad térmica mediante la ecuación parabólica dada en [33, 34,37]. Los valores de las temperaturas calculadas por ambos métodos coinciden bastante bien, lo que supone una excelente validación del modelo FEM construido. 


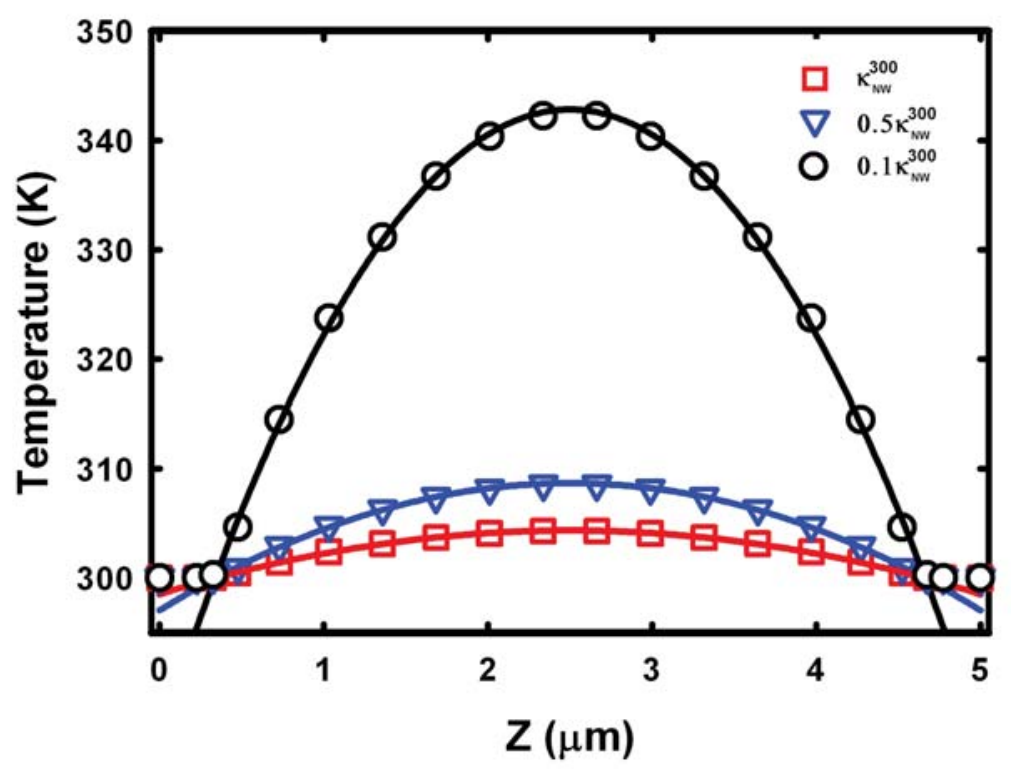

Figura 3.10: Temperatura alcanzada en el punto del NW en el que se focaliza el haz láser representada en función de la posición del punto de focalización del haz láser a lo largo del NW. El NW es de silicio con un diámetro de $37 \mathrm{~nm}$ y $5 \mu \mathrm{m}$ de longitud, estando $4.5 \mu \mathrm{m}$ suspendidos y $0.25 \mu \mathrm{m}$ por cada lado en contacto con el substrato en condición de contacto térmico perfecto. La potencia del haz incidente es de $200 \mu \mathrm{W}$ y su longitud de onda de $514.5 \mathrm{~nm}$. Las lineas son los ajustes obtenidos con la ecuación dada por Tsu et al. en [33] para cada conductividad. 


\subsubsection{Efecto de la posición que ocupa el NW dentro del spot del haz láser}

Hasta ahora se ha estudiado la temperatura inducida en NWs iluminados por un haz láser en función de sus propiedades intrínsecas y la forma en que disipan la energía absorbida, pero aún resta de estudiar un factor muy relevante en la interacción láser-NW; la densidad de potencia de un haz láser como los utilizados en técnicas de caracterización microscópicas no es homogénea en la zona de iluminación o spot. Esta densidad de potencia es mucho mayor en el centro del spot láser y decae rápidamente al alejarse de él siguiendo una curva gaussiana dada por la ecuación 3.2. Cuando la muestra es mucho más grande que la zona donde se concentra la mayor parte de la potencia del haz, esta distribución no homogénea de la potencia depositada en la muestra no tiene un efecto relevante en la señal obtenida. Sin embargo, cuando la muestra es mucho más pequeña que el área en el que se deposita la mayoría de la energía del haz láser, esta distribución no homogénea de la potencia juega un papel fundamental, ya que la posición donde se sitúa la muestra en el interior del spot va a determinar la intensidad con la que es iluminada por el láser. Por otro lado, el diámetro del NW está por debajo del límite de difracción para longitudes de onda visibles, y por tanto no pueden ser resueltos en microscopia óptica; debido a esta limitación, la alineación experimental del NW con el foco del haz láser no es algo que se pueda obtener directamente.

Para entender mejor este efecto de nanoescala, se ha simulado la temperatura alcanza por un NW de silicio liso en función de la posición que ocupa dentro del área de iluminación de un haz láser, pudiéndose observar el resultado en la figura 3.11. Puede comprobarse como existen unas diferencias de temperatura muy grandes dependiendo de la posición relativa del NW con respecto al centro del foco del haz láser. Otro efecto relevante en la temperatura alcanzada por el NW es la posición a lo largo del NW en la que se focaliza el haz láser. Podemos observar este efecto en la figura 3.11 b). donde se observa que si el NW es iluminado en la proximidad de uno de sus extremos, la temperatura puede ser muy diferente de si es iluminado en el centro del NW. Estos resultados son muy relevantes cuando se realizan medidas ópticas mediante un haz láser en grupos de NWs. Cuando un grupo de NWs es iluminado simultáneamente por un haz láser, la intensidad de la 


\section{ESTUDIO TEÓRICO DE LA DISTRIBUCIÓN DE TEMPERATURA Y SU EFECTO EN LA SEÑAL RAMAN DE NWS SEMICONDUCTORES BAJO UN HAZ LÁSER}
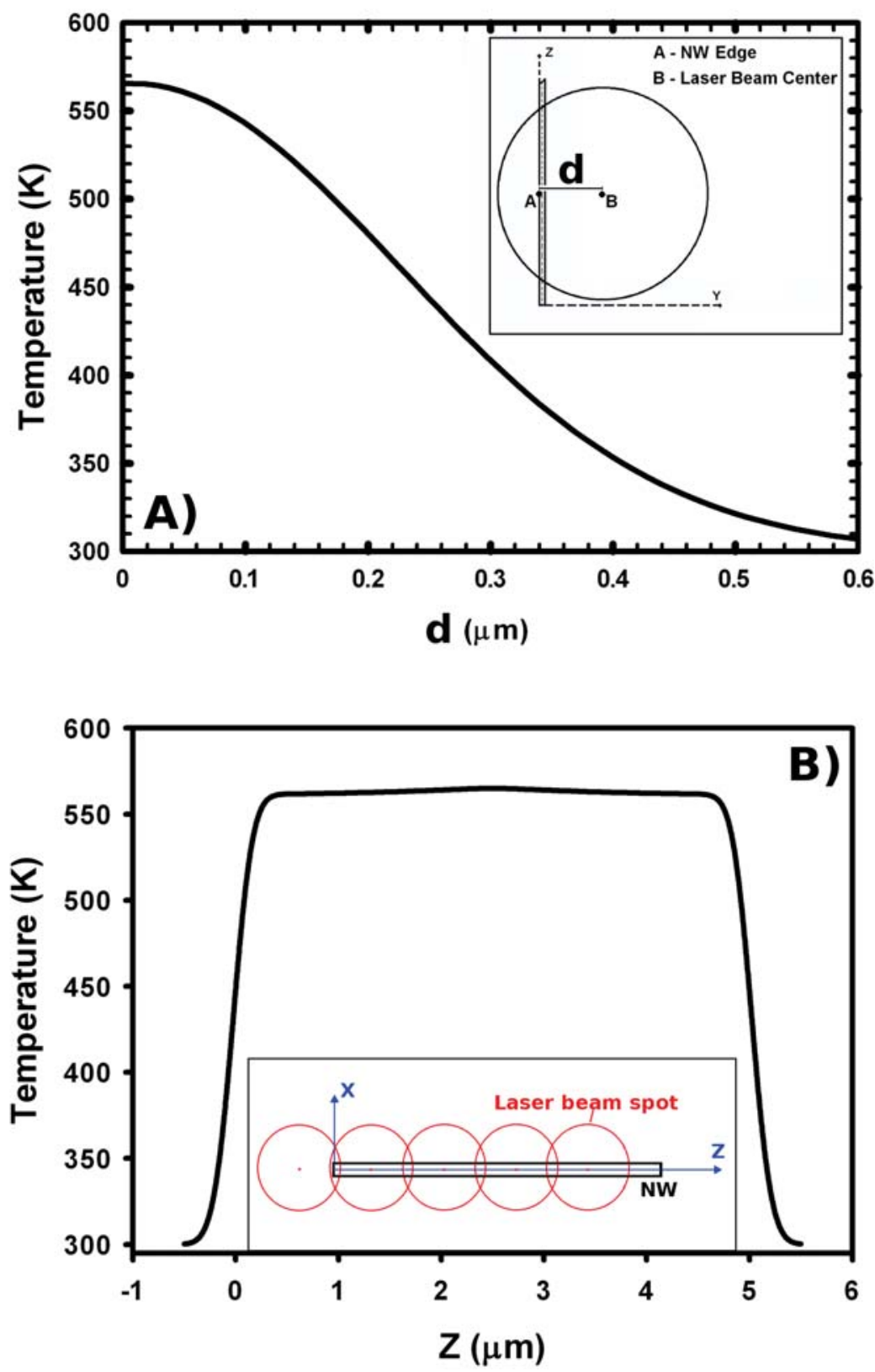

Figura 3.11: A) Temperatura inducida en un NW de $37 \mathrm{~nm}$ de diámetro y $5 \mu \mathrm{m}$ de longitud en función de la posición que el NW ocupa dentro del spot del haz láser (ver recuadro interno para una mejor comprensión de la simulación). B) Temperatura inducida en un NW de $37 \mathrm{~nm}$ de diámetro y $5 \mu \mathrm{m}$ de longitud en función de la posición a lo largo del NW en la que está enfocado el haz láser. El láser tiene $1 \mu \mathrm{m}$ de diámetro efectivo, una longitud de onda de $514.5 \mathrm{~nm}$ y $200 \mu \mathrm{W}$ de potencia en ambos casos. 
señal y la temperatura de cada NW dependerá de su configuración espacial en el interior del área de iluminación o spot del haz láser, además de sus dimensiones y sus características superficiales, además de a posición a lo largo del NW donde está enfocado el haz láser. Todos estos factores de los que depende la temperatura de los NWs iluminados tienen como consecuencia la existencia de una distribución muy inhomogénea de temperaturas en la zona de la que se obtiene la señal; sin embargo esta inhomogeneidad no proviene de grandes gradientes en el interior de cada NW, sino de las muy diferentes temperaturas que puede alcanzar cada NW iluminado. Para ilustrar este efecto se han construido dos modelos en los que intervienen conjuntos de NWs. En el primero se ha simulado la temperatura alcanzada por cuatro NWs de silicio liso, idénticos y paralelos entre sí por simplicidad, pudiéndose observar el resultado en la figura 3.12 A) y B). Cada NW del conjunto alcanza unas temperaturas muy diferentes de los otros dependiendo de su posición dentro del área de iluminación, además frente a un pequeño cambio del punto de focalización (100 nm en $\mathrm{x}$ ), las temperaturas de cada NW son completamente diferentes al paso anterior. Esto implica que la señal óptica recolectada de estas muestras ( en Raman, PL, etc...) va a incorporar una gran indeterminación, ya que se va a componer de la muy distinta contribución aportada por cada NW iluminado.

En la segunda simulación se han tomado dos NWs idénticos silicio lisos de $37 \mathrm{~nm}$ diámetro y $5 \mu$ de longitud, que se encuentran cruzados de forma asimétrica, donde uno está sobre otro, pudiéndose observar los efectos de sombra y las diferentes temperaturas alcanzadas por cada NW en función de la posición en donde se encuentra focalizado el haz láser. De nuevo podemos observar las muy diferentes temperaturas que pueden alcanzar los dos NWs en función de la posición del haz láser pese a estar en iluminados de una forma, a priori, muy similar. Es importante destacar que estos cálculos están realizados asumiendo que no existen efectos de polarización en la interacción láser-NW. Sin embargo, posteriormente se mostrara que cuando el haz láser está linealmente polarizado existe un importante efecto de polarización en la interacción láser-NW. Pese a esto, todas las simulaciones realizadas en este apartado son válidas siempre que el eje longitudinal del NW esté alineado con el campo eléctrico del haz láser o bien siempre que el haz láser que ilumina la muestra no esté linealmente polarizado. 


\section{ESTUDIO TEÓRICO DE LA DISTRIBUCIÓN DE TEMPERATURA Y SU EFECTO EN LA SEÑAL RAMAN DE NWS SEMICONDUCTORES BAJO UN HAZ LÁSER}
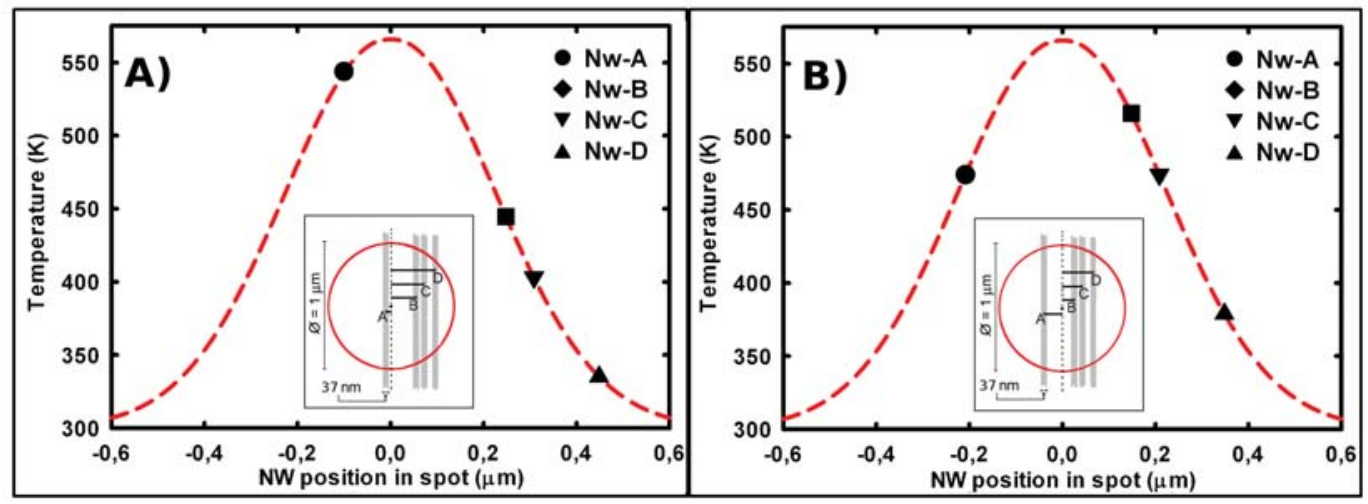

Figura 3.12: Temperaturas calculadas para un conjunto de NWs de silicio lisos idénti$\cos$ (37 nm de diámetro, $5 \mu \mathrm{m}$ de longitud) iluminados simultáneamente por un haz láser de $514.5 \mathrm{~nm}$ de longitud de onda y $200 \mu \mathrm{W}$ de potencia. Se muestran dos configuraciones espaciales de estos NWs, A) y B) separadas por un salto de $100 \mathrm{~nm}$ entre puntos de focalización de haz láser. Puede apreciarse las diferentes temperaturas que se inducen en ambas situaciones pese a la utilización de la misma muestra y la misma fuente de iluminación

\subsection{Influencia de la temperatura alcanzada por distin- tos NWs de silicio en la señal Raman}

El análisis teórico por elementos finitos del comportamiento térmico de la interacción láser-NWs, ha puesto de manifiesto la gran dependencia de la temperatura alcanzada en NWs iluminados por un mismo haz láser con las dimensiones, características de superficie y posición relativa dentro de la zona de iluminación. La temperatura alcanzada por los NWs iluminados va a representar un papel muy relevante cuando se trate de analizar el espectro Raman de los NWs. Los principales parámetros que caracterizan el espectro Raman son la intensidad, la frecuencia a la que aparece el pico Raman y la anchura a media altura de dicho pico Raman. Como ya se ha descrito en el capitulo 1, estos parámetros son sensibles a la temperatura a la que se encuentra la muestra. La temperatura excita los niveles de energía del cristal, lo que introduce términos de anharmonicidad mecánicos en el Hamiltoniano vibracional que describe la energía de la red $[53,54]$. Dado que las transiciones que desencadenan la emisión Raman suceden desde niveles excitados térmicamente, éstas tienen una menor energía que las transiciones que suceden desde el estado 

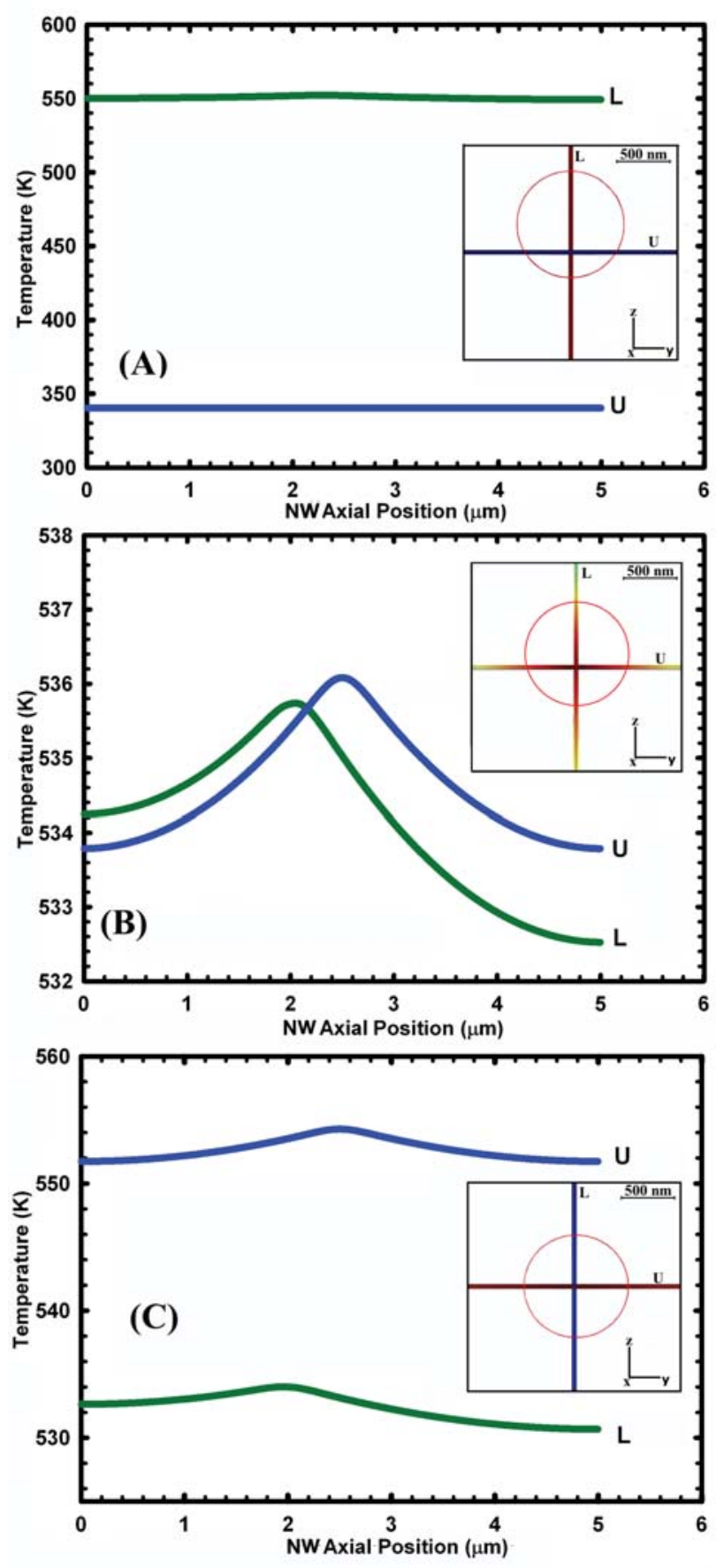

Figura 3.13: Temperaturas alcanzadas por dos NWs idénticos de $37 \mathrm{~nm}$ de diámetro y $5 \mu \mathrm{m}$ de longitud que se cruzan a 90 grados, estando unos de ellos (U) justo por encima del otro (L) y sin contacto térmico entre ambos (ver esquema de la figura 3.2 C) ). Se muestran 3 configuraciones diferenciadas únicamente por la posición en la que se está focalizando el haz láser en el eje z, estando A) a focalizado a $400 \mathrm{~nm}$ del punto de corte entre los NWs, B) a $140 \mathrm{~nm}$ y C) a $80 \mathrm{~nm}$ (ver esquemas en las figuras como guía aproximada para el ojo). 


\section{ESTUDIO TEÓRICO DE LA DISTRIBUCIÓN DE TEMPERATURA Y SU EFECTO EN LA SEÑAL RAMAN DE NWS SEMICONDUCTORES BAJO UN HAZ LÁSER}

no excitado y por tanto la frecuencia vibracional que caracteriza dicha energía se desplaza a valores más bajos, expandiéndose simultáneamente en un numero finito de valores permitidos de forma simétrica alrededor de la frecuencia característica [53], lo que se traduce en un espectro Raman con el pico desplazado hacia bajas frecuencias y más ancho cuanto mayor es la temperatura de la muestra. Por tanto, dependiendo de la geometría, características superficiales y posición en el área de excitación de los NWs, el espectro Raman que obtendremos de ellos podría no ser idéntico, pese a ser de idéntico el material, por el efecto de las diferentes temperaturas inducidas en ellos por el haz láser.

Otro punto a tener en cuenta cuando se analiza el espectro Raman de los NWs viene determinado exclusivamente por sus reducidas dimensiones. La longitud de onda característica de los fonones ópticos es del orden de los nanometros, y por tanto es comparable a las dimensiones del diámetro de los NWs, pudiendo aparecer efectos de confinamiento fonónico que afecten al espectro Raman como sucede en nanocristales $[55,56]$. El efecto del confinamiento es un desplazamiento hacia bajas frecuencias del pico Raman, un ensanchamiento de dicho pico y una notable asimétrica es su forma dada por la expansión a frecuencias vibracionales de baja energía [24]; es decir, la temperatura y el confinamiento fonónico tienen un efecto similar en el espectro Raman salvo por la ruptura de la simetría que introduce el confinamiento por la deformación del espectro. Sin embargo, para que los efectos de confinamiento fonónico comiencen a ser apenas perceptibles, los diámetros de los NWs tienen que ser menores a $20 \mathrm{~nm}[24,57]$, pasando desapercibidos para diámetros mayores.

Ambos efectos, temperatura y confinamiento fonónico, pueden estar presentes cuando se analizan NWs semiconductores mediante espectroscopia Raman. Si se realizan los experimentos en conjuntos de NWs, ambos efectos distorsionan la señal recibida; esta señal estará formada por la contribución de cada NW, y cada NW estará a una temperatura diferente tal y como hemos comprobado con la simulaciones FEM y bajo condiciones de excitación diferentes. Además, si las dimensiones de los NWs caen por debajo del límite para el cual el confinamiento fonónico comienza a ser relevante, la contribución de cada NW también será diferente.

Para entender el papel jugado por estos efectos en el espectro Raman que se obtiene de conjuntos de NWs, uno puede simular tres escenarios diferentes que dan cuenta 


\subsection{Influencia de la temperatura alcanzada por distintos NWs de silicio en la}

señal Raman

de los efectos de temperatura y confinamiento en el espectro Raman, recurriendo para ello al formalismo desarrollado por Ritcher et al. [6] y posteriormente adaptado a estructuras cilíndricas por Campbel et al. [7], y que permite simular el espectro Raman en NWs. Teniendo en cuenta esto, los escenarios posibles estarán descritos por:

1. Todos los NWs de la muestra iluminada por el haz láser tienen unos diámetros para los cuales el efecto de confinamiento fonónico es relevante, i.e. $D_{N W}<20 n m$, y la temperatura inducida en ellos por el haz láser es irrelevante.

2. Todos los NWs de la muestra iluminada por el haz láser tienen unos diámetros para los cuales el efecto de confinamiento fonónico es relevante, i.e. $D_{N W}<20 \mathrm{~nm}$, y además el láser induce una temperatura en los NWs no despreciable.

3. Todos los NWs de la muestra iluminada por el haz láser tienen unos diámetros para los cuales el efecto de confinamiento fonónico es irrelevante, i.e. $D_{N W}>20 \mathrm{~nm}$, y además el láser induce una temperatura en los NWs no despreciable.

La posibilidad de que coexistan en la muestra NWs muy por debajo del límite de confinamiento y NWs con diámetros mayores está excluida dado que la señal de los primeros es mucho más débil, y su efecto pesa muy poco en la señal global. Por otro lado, asumimos que estos tres escenarios resumen todas las posibilidades que pueden deformar el espectro Raman en NWs semiconductores siempre y cuando estos no hayan sido dopados o fuertemente tensionados $[58,59,60]$, efectos que también contribuyen a la forma final del espectro Raman.

\section{1 confinamiento fonónico sin calentamiento $(D<20 \mathrm{~nm})$}

La primera posibilidad estudiada es que todos los NWs iluminados por el haz láser tengan un tamaño tal que los efectos de confinamiento fonónico sean muy relevantes, y por tanto que sus tamaños estén por debajo del límite de $20 \mathrm{~nm}$; además, se considera la hipótesis de que se puede obtener una señal mensurable con una potencia tal que no se induce calentamiento en los NWs. A efectos de simplificar el 


\section{ESTUDIO TEÓRICO DE LA DISTRIBUCIÓN DE TEMPERATURA Y SU EFECTO EN LA SEÑAL RAMAN DE NWS SEMICONDUCTORES BAJO UN HAZ LÁSER}

estudio, pero sin perder generalidad por ello, se han tomado solo cuatro NWs lisos de silicio paralelos de 6, 7,11 y $12 \mathrm{~nm}$ de diámetro y $5 \mu \mathrm{m}$ de longitud dispuestos en dos configuraciones espaciales diferentes (ver figura 3.14 A) y B) ). Utilizando la relación fenomenológica dada en las referencias [6] y [7], la cual tiene en cuenta el efecto del confinamiento fonónico en estructuras cilíndricas, se ha calculado la intensidad Raman de cada NW de acuerdo con [7]:

$$
I(\omega) \propto \int e^{\left(-\frac{q^{2} D^{2}}{16 \pi^{2}}\right)} \frac{2 \pi q d q}{\left(\omega-\omega(q)^{2}+\left(\frac{\Gamma_{0}}{2}\right)^{2}\right.}
$$

Donde se ha utilizado una función gaussiana de localización como la descrita en [6], $\Gamma_{0}$ es la anchura a media altura del pico Raman para el silicio cristalino, $q$ es el vector de onda dado en el rango $q \in[0, \pi / a]$, siendo $a$ el parámetro de red del silicio cristalino , $D$ corresponde al diámetro de cada NW, y por último $\omega(q)$ es la relación de dispersión fononica, que para el silicio en ausencia de calentamiento inducido por el haz láser (i.e. $T=300 \mathrm{~K}$ ) puede aproximarse como [61]:

$$
\left.\omega(q)=(1,714+\cos (\pi q / 2)) \times 10^{5}\right)^{1 / 2}\left(\mathrm{~cm}^{-1}\right)
$$

La intensidad Raman emitida por cada NW dependerá además de la potencia láser con la que es excitado cada NW; existe una dependencia lineal de la intensidad Raman con la potencia absorbida por los NWs siempre que la longitud de los NW sea mayor a $1 \mu \mathrm{m}$ [62]. Dada la distribución inhomogénea de la potencia en el área de iluminación del haz láser (láser de $632.8 \mathrm{~nm}$ de longitud de onda y una potencia en la muestra de $1.8 \mathrm{~mW}$ con distribución gaussiana de la intensidad), esto se traduce en que la intensidad con la que contribuye cada NW a la señal final depende de la posición en la que se encuentra dentro del área iluminada por el haz láser. Si todos los NWs son iguales esto no tiene mucha relevancia, dado que la señal Raman de todos ellos tendrá las mismas características dadas por la frecuencia de pico Raman, anchura a media altura y relación de asimétria entre parte derecha e izquierda del pico Raman. Sin embargo, la situación real más probable en una muestra de varios NWs, es que las dimensiones de los NWs iluminados difieran ligeramente, iluminándose simultáneamente NWs de diámetros diferentes. Cuando esto sucede, la posición de cada NW en el área de iluminación es determinante en la forma 


\subsection{Influencia de la temperatura alcanzada por distintos NWs de silicio en la} señal Raman

del espectro Raman que se obtiene de la muestra. Puede observarse este efecto en las dos configuraciones mostradas en las figuras 3.14 A) y B), donde los mismos NWs son iluminados por el mismo haz láser, pero en cada una de las figuras los NWs han sido redistribuidos en el área de iluminación del haz láser. El espectro resultante en cada caso presenta la forma típica que se obtendría para el espectro Raman de un NW en el que el confinamiento fonónico es muy relevante. De hecho, este espectro resultante puede ajustarse al espectro correspondiente a un NW de silicio con un diámetro determinado en cada caso, siento $8 \mathrm{~nm}$ para la configuración espacial mostrada en 3.14 A) y $10 \mathrm{~nm}$ para la configuración espacial 3.14 B). Estos resultados tan diferentes se obtienen del mismo grupo de NWs y bajo las mismas condiciones de iluminación, lo que demuestra que el análisis del espectro Raman en grupos de NWs que presentan confinamiento fonónico puede conducir a una interpretación errónea de los datos experimentales, incluso cuando en éstos no está presente ningún efecto de temperatura. Por otro lado, este resultado también explica por que falla la determinación del diámetro de un conjunto de NWs a través del ajuste al espectro Raman, como se apuntó en [20].

\section{Confinamiento fonónico + temperatura}

En apartado anterior, ha quedado patente que cuando se obtiene el espectro Raman en conjuntos de NWs que presentan confinamiento fonónico es difícil realizar una correcta interpretación de la señal obtenida. Si además introducimos el efecto de la temperatura que se induce en un NW cuando es iluminado, la incertidumbre introducida por la posición relativa que ocupan los NWs en el área iluminada por el haz láser puede ser aún mayor.

Cuando un NW es calentado por el haz láser, la ecuación 3.9 utilizada para calcular la intensidad Raman emitida debe ser modificada para incluir la dependencia de relaciones de dispersión fonónicas, $\omega(q)$, y de la anchura a media altura, $\rho$, con la temperatura. Las relaciones de dispersión dependientes de la temperatura pueden expresarse como $[30,63,64]$ :

$$
\omega(q, T)=\omega(q)+\triangle_{t e}(T)+\triangle_{a}(T)
$$




\section{ESTUDIO TEÓRICO DE LA DISTRIBUCIÓN DE TEMPERATURA Y SU EFECTO EN LA SEÑAL RAMAN DE NWS SEMICONDUCTORES BAJO UN HAZ LÁSER}
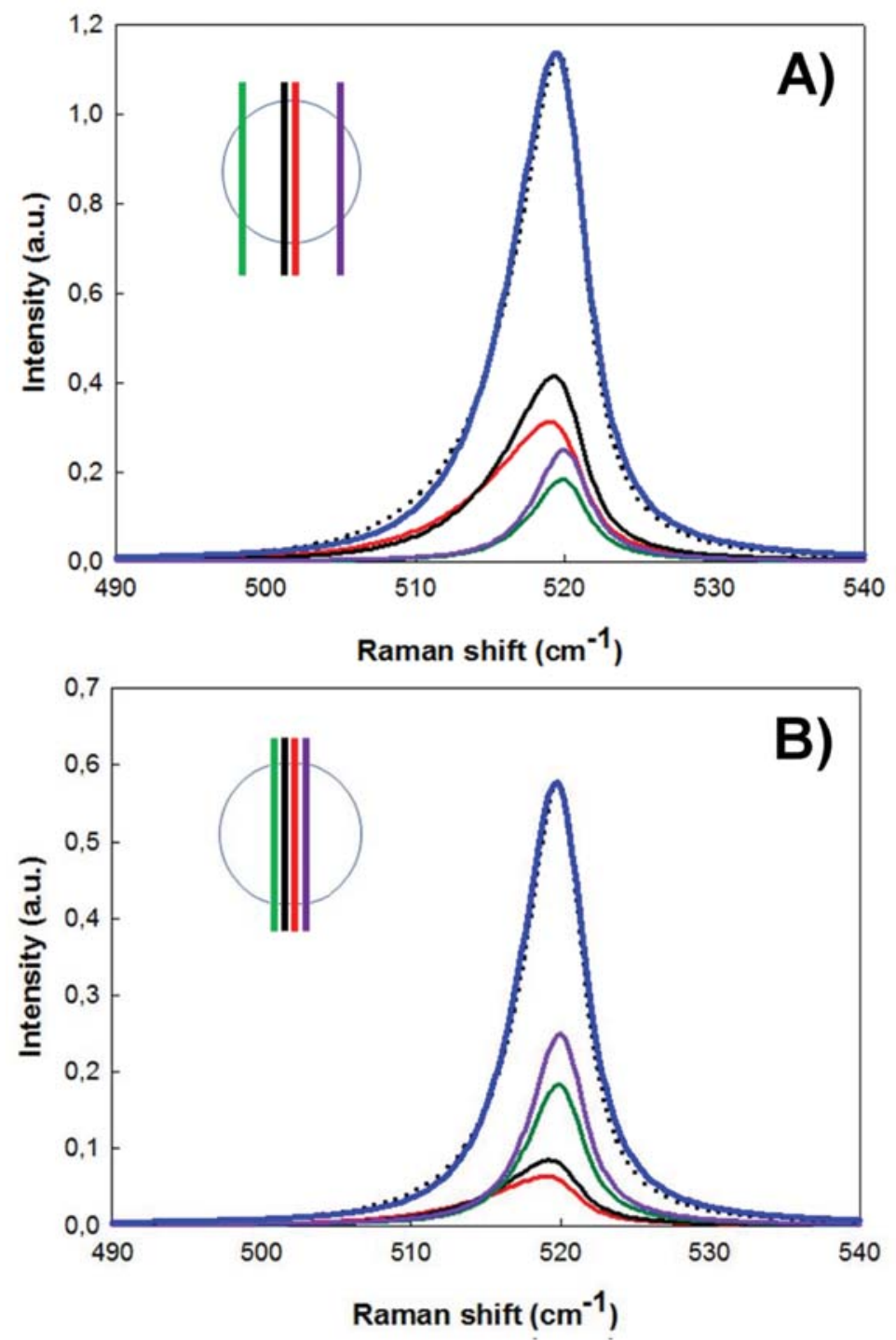

Figura 3.14: Espectro Raman calculado para un conjunto de NWs de silicio liso de 5 $\mu$ m de longitud y unos diámetros de $11,6,7$ y $12 \mathrm{~nm}$ respectivamente en dos configuraciones espaciales diferentes ( A) - $0.35,-0.01,0.01$, y $0.35 \mu \mathrm{m}$; B) $-0.05,-0.01$, 0.015 y $0.05 \mu \mathrm{m}$ del centro del área de iluminación del haz láser respectivamente, el diámetro efectivo del haz láser es $1 \mu \mathrm{m}$ ). En cada figura se muestra la configuración espacial dentro del área de iluminación. En cada caso la curva punteada corresponde al espectro calculado para un único NW de $8 \mathrm{~nm}$ de diámetro para la configuración A)y $10 \mathrm{~nm}$ para la configuración B), mostrando como la redistribución espacial de NWs puede ser confundida con la señal procedente de un único NW de un diámetro determinado. 


\subsection{Influencia de la temperatura alcanzada por distintos NWs de silicio en la} señal Raman

Donde $\triangle_{t e}$ es la contribución dada por el coeficiente de expansión térmica y $\triangle_{a}$ la contribución anharmonica. El primero de ellos se describe como:

$$
\triangle_{t e}(T)=\omega_{0}\left(e^{-3 \gamma \int_{T_{0}}^{T} \alpha^{\prime}\left(T^{\prime}\right) d \alpha^{\prime}}-1\right)
$$

Donde $\gamma$ es el parámetro de Grüneisen y $\alpha$ el coeficiente de expansión térmica. La contribución debida al decaimiento anharmonico de los fonones ópticos en acústicos está dada como:

$$
\begin{gathered}
\triangle_{a}(T)=A\left(1+\frac{2}{e^{\hbar \omega / 2 K T}-1}\right)+ \\
+B\left(1+\frac{3}{e^{\hbar \omega / 3 K T}-1}+\frac{3}{\left(e^{\hbar \omega / 3 K T}-1\right)^{2}}\right)
\end{gathered}
$$

Por otro lado, la anchura a media altura del pico Raman también depende de los procesos anharmonicos de decaimiento fonónico, estando caracterizada por :

$$
\begin{gathered}
\Gamma(T)=\Gamma_{0}+C\left(1+\frac{2}{e^{\hbar \omega / 2 K T}-1}\right)+ \\
+D\left(1+\frac{3}{e^{\hbar \omega / 3 K T}-1}+\frac{3}{\left(e^{\hbar \omega / 3 K T}-1\right)^{2}}\right)
\end{gathered}
$$

Por último, los parámetros anharmonicos $A, B, C$ y $D$, se suponen idénticos a los calculados para el silicio volúmico, i.e $A=-2,96, B=-0,174, C=1,295$ y $D=-0,105[65]$; la utilización de estos parámetros está justificada dado que Doerk et al. no observaron cambios en los parámetros de anharmonicidad en NWs de silicio en comparación con los del silicio volúmico [30].

Con estas modificaciones, se ha calculado el espectro resultante de iluminar un conjunto de NWs de silicio liso de $5 \mu \mathrm{m}$ de longitud y diámetros 10, 11, 12 y $20 \mathrm{~nm}$. Se han escogido estos diámetros por que combinan bien los efectos de temperatura, confinamiento e intensidad cuando son iluminados con un haz láser de $632.8 \mathrm{~nm}$ de longitud de onda y una potencia en la muestra de $1.8 \mathrm{~mW}$. Los NWs por debajo de $12 \mathrm{~nm}$ son tan finos que resultan casi transparentes para este haz láser, resultando en una potencia absorbida muy baja y por tanto en una muy baja temperatura inducida en ellos. El NW de $20 \mathrm{~nm}$ tiene un efecto de confinamiento más débil, pero a cambio en el se inducen temperaturas mucho más elevadas. En conjunto se obtiene 


\section{ESTUDIO TEÓRICO DE LA DISTRIBUCIÓN DE TEMPERATURA Y SU EFECTO EN LA SEÑAL RAMAN DE NWS SEMICONDUCTORES BAJO UN HAZ LÁSER}

una buena combinación de los efectos de confinamiento y temperatura en NWs con confinamiento fonónico. La temperatura inducida en cada NW se obtiene mediante elementos finitos, pero para ello es necesario estimar la conductividad térmica en NWs por debajo de los $20 \mathrm{~nm}$, para los que el modelo desarrollado en el capítulo 2 no es válido. Sin embargo, a través de la ecuación 2.33 para estimar la conductividad térmica a una temperatura puede realizarse un extrapolación. A partir de 300 $\mathrm{K}$, cuanto menor es el diámetro menor es la variación de la conductividad térmica con la temperatura. Por otro lado, a muy alta temperatura el efecto de las dimensiones de los NWs va perdiendo peso (ver figura 2.12) en la conductividad térmica, cometiendo por tanto un error menor al extrapolar hacia diámetros menores a 20 nm. Utilizando la expresión 2.33 para $1500 \mathrm{~K}$, podemos estimar la conductividad térmica de los NWs con diámetros entre 10 y 12 nm entre 1 y 1.5 W/mK a $300 \mathrm{~K}$. Utilizando estos valores a falta de otros más precisos, se ha calculado sus temperaturas para dos configuraciones espaciales diferentes, y sus espectros resultantes se muestran en la figura 3.15 A) y 3.15 B). En ellas podemos observar el efecto dominante de la posición del NW más grueso, pero también como los NWs más finos asimetrizan el espectro final, de tal modo que el espectro resultante puede malinterpretarse y atribuirse a un un solo NW o un conjunto idéntico de NWs de $11 \mathrm{~nm}$ (configuración A) o de 10 nm de diámetro (configuración B) que no son calentados por el haz láser.

\section{Efectos de temperatura sin confinamiento $(D>20 \mathrm{~nm})$}

Por último, vamos a considerar muestras formadas por NWs con diámetros por encima del límite de confinamiento fonónico. En este caso las temperaturas alcanzadas por los NWs pueden ser mucho más altas que para NWs de diámetros pequeños; la eficiencia de absorción es muy baja para NWs de diámetros por debajo de límite de confinamiento para las longitudes de onda analizadas (ver figura 3.2 C) ), y junto al mayor volumen de material, se traducen en una mayor energía absorbida del haz láser que ilumina los NWs con diámetros por encima del límite de confinamiento. Por otro lado, se ha mostrado como la temperatura de los NWs depende enormemente de la posición que ocupan estos en el área iluminada por 

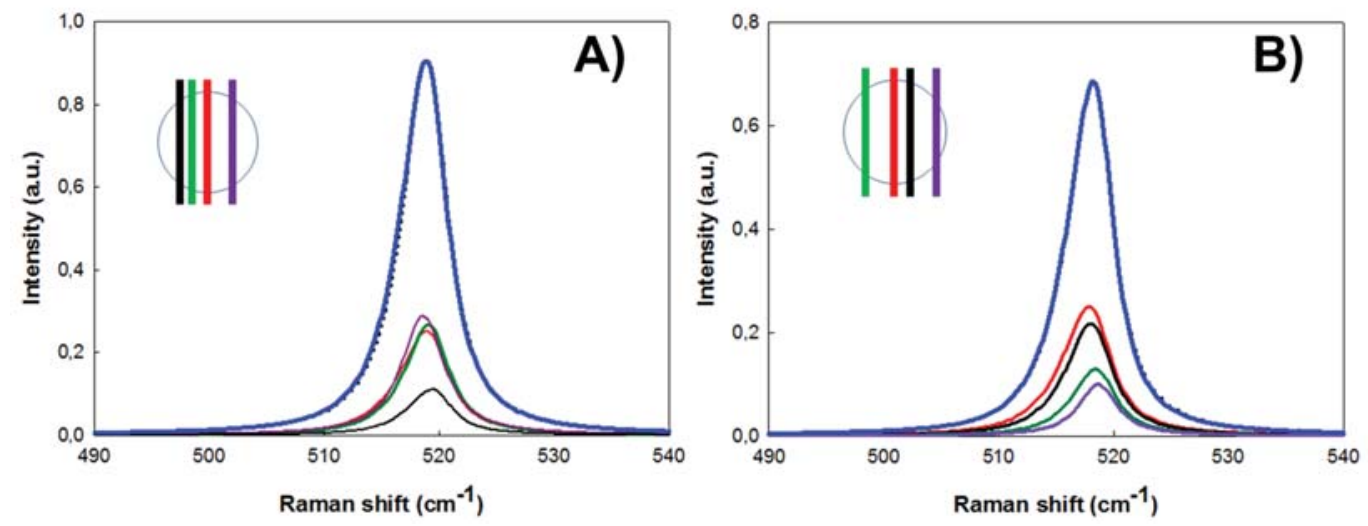

Figura 3.15: Espectros Raman simulados para un conjunto de NWs iluminados por un haz láser de $632.8 \mathrm{~nm}$ de longitud de onda y una potencia en la muestra de $1.8 \mathrm{~mW}$. Los NWs son de silicio, lisos y con unos diámetros de 12, 10, 11 y $20 \mathrm{~nm}$ respectivamente. En la figura A) están situados a -0.30, 0, 0.15, y $0.4 \mu \mathrm{m}$ del centro del spot láser; B) Los NWs están situados a $-0.25,-0.17,0$ y $0.28 \mu \mathrm{m}$ del centro del spot láser. las curvas punteadas representan los ajustes a un único NW que no se calienta de $11 \mathrm{~nm}$ para la configuración A) y de $10 \mathrm{~nm}$ para la configuración B)

el haz láser (ver figura 3.11 A) ), lo que va a tener un efecto en el espectro Raman recolectado de la muestra. Para observar esto, se ha simulado la intensidad Raman de cuatro hilos idénticos de $25 \mathrm{~nm}, 5 \mu \mathrm{m}$ de longitud e iluminados por un haz láser con una longitud de onda de $632.8 \mathrm{~nm}$ y una potencia en la muestra de $1.8 \mathrm{~mW}$, mediante la ecuación 3.10, convenientemente modificada por $3.11 \mathrm{y}$ 3.14 para dar cuenta de la temperatura inducida en los NWs. La temperatura y la potencia en cada NW se extrae de un análisis por elementos finitos, de modo que obtenemos una distribución de temperaturas en los NWs en función de su posición equivalente a la mostrada en la figura 3.13. En la figura 3.16 A) y B) se muestran las intensidades Raman obtenidas de esta forma para dos configuraciones espaciales diferentes. Puede observarse en la figura 3.16 A), como el espectro de cada NW es diferente, teniendo una posición de pico Raman y anchura a media altura diferentes, efecto causado por estar sometidos a unas condiciones de excitación diferentes; además, dado que el confinamiento fonónico es irrelevante, los espectros individuales son prácticamente simétricos. Sin embargo, su contribución conjunta resulta en un espectro cuyo pico Raman esta desplazado $4 \mathrm{~cm}^{-1}$, está ensanchado 


\section{ESTUDIO TEÓRICO DE LA DISTRIBUCIÓN DE TEMPERATURA Y SU EFECTO EN LA SEÑAL RAMAN DE NWS SEMICONDUCTORES BAJO UN HAZ LÁSER}

y su perfil es asimétrico. Es decir, el espectro Raman de una muestra compuesta por NWs que individualmente no presentan confinamiento fonónico puede malinterpretarse, ya que el espectro resultante muestra las características de muestras en las que si que existe confinamiento. Por otro lado, si se redistribuyen los NWs en el área de iluminación, el espectro resultante cambia completamente. En la figura 3.16 B) puede observarse como incluso el pico Raman puede llegar a separarse en dos, un resultado que no puede ser explicado sino es a través de la gran diferencia en la temperatura alcanzada por los NWs dependiendo de la posición en la que se encuentran dentro del haz láser. Como veremos más adelante, este resultado aparece cuando se realizan medidas experimentales mediante espectroscopia Raman en muestras compuestas por conjuntos de NWs, y mediante este análisis encuentran una explicación satisfactoria.

\subsection{Conclusión}

A lo largo de este capítulo hemos podido comprobar como los efectos térmicos en la interacción láser-NWs tiene unas características muy diferentes a lo que uno está acostumbrado a observar en escala macroscópica. La temperatura que se induce en los NWs no es solo dependiente de la potencia nominal de haz incidente, si no que es muy dependiente de las dimensiones del NW, la longitud de onda de haz, las condiciones de disipación y las características de la superficie de los NWs. Además, el hecho de que el diámetro de los NWs sea menor que la longitud de onda de excitación y que el área de iluminación del láser, el cual no tiene una distribución de potencia homogénea, tiene un efecto determinante en las temperaturas inducidas en los NWs. A consecuencia de todo ello, el análisis del espectro Raman en conjuntos de NWs encierra una gran indeterminación que puede acarrear interpretaciones erróneas de la señal recibida. Típicamente, se ha recurrido a analizar el espectro Raman en conjuntos de NWs que tienen una baja señal individual, especialmente cuando sus diámetros están por debajo del límite de confinamiento fonónico. En estos casos, incluso cuando se consigue eliminar el efecto de la temperatura, la señal recolectada sigue encerrando una gran indeterminación debido a las diferentes condiciones de excitación de cada NW simultáneamente iluminado. Cuando se realiza el análisis en conjuntos de NWs en los que no hay confinamiento, 


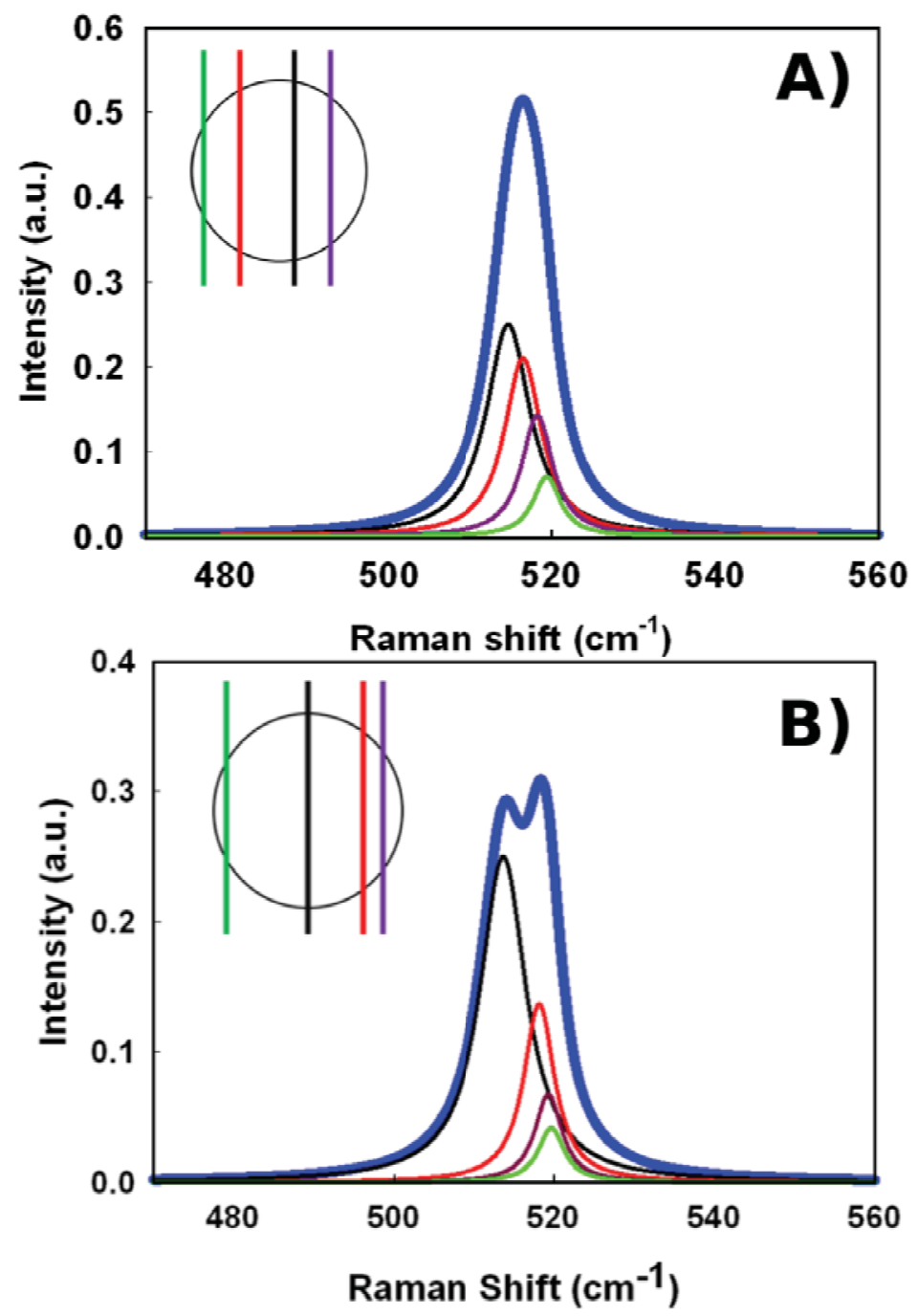

Figura 3.16: Espectro Raman simulado para un conjunto de NWs de $25 \mathrm{~nm}$ de diámetro y $5 \mu \mathrm{m}$ de longitud situados a una distancia del centro del área de iluminación de $-0.40,-0.20,0.10$ y $0.30 \mu \mathrm{m}$ para A) y $-0.45,-0.01,0.30$ y $0.40 \mu \mathrm{m}$ para B). Los espectros resultantes muestan diferencias notables dependiendo de como están distribuidos los NWs en el área de iluminación, pese a que la muestra iluminada es la misma. Observesé como incluso aparece una separación del espectro Raman en dos picos únicamente por el efecto de las diferentes temperaturas alcanzadas por cada NW. 


\section{ESTUDIO TEÓRICO DE LA DISTRIBUCIÓN DE TEMPERATURA Y SU EFECTO EN LA SEÑAL RAMAN DE NWS SEMICONDUCTORES BAJO UN HAZ LÁSER}

no se puede asumir directamente que bajando la potencia del haz láser por debajo de un determinado umbral va a garantizar que no se eleve la temperatura de los NWs, ya que dada la enorme dependencia de la absorción de energía con el diámetro, lo que para unos NWs del conjunto puede ser una potencia baja, para otros puede ser suficiente para elevar considerablemente su temperatura. Por tanto, para realizar un correcto análisis experimental de la señal Raman proveniente de NWs semiconductores, lo más recomendable es analizar NWs individuales y verificar que en ellos no se induce una temperatura capaz de modificar su espectro Raman característico. 


\subsection{Bibliografía}

[1] Cui Y, Lieber C.M. Functional Nanoscale Electronic Devices Assembled using Silicon Nanowire Building Blocks. Science, 2001;291, 851

[2] Hochbaum A.I, Chen R, Delgado R.D, Liang W, Garnett E.C, Najarian M, Majumdar A, Yang P. Enhanced thermoelectric performance of rough silicon nanowires. Nature, 2008;451, 163

[3] Kayes B.M, Atwater H.A, Lewis N.S. Comparison of the device physics principles of planar and radial p-n junction nanorod solar cells. J. Appl. Phys. 2005;97, 114302

[4] Nakayama Y, Pauzauskie P. J, Radenovic A, Onorato R. M, Saykally R. J, Liphardt J, Yang P. Tunable nanowire nonlinear optical probe. Nature 2007;447, 1098 [5] Singh N, Buddharaju K.D, Manhas S.K, Agarwal A, Rustagi S.C, Lo G.Q, Balasubramanian N, Kwong D.L. Si, SiGe Nanowire Devices by Top-Down Technology and Their Applications. IEEE Trans.Electron Devices 2008;55, 3107

[6] Richter H, Wang Z.P, Ley L.The one phonon Raman spectrum in microcrystalline silicon. Solid St. Commun. 1981;39, 625

[7] Campbell I.H, Fauchet P.M. The Effects Of Microcrystal Size And Shape On The One Phonon Raman Spectra Of Crystalline Semiconductors. Solid St. Commun. 1986;58, 739

[8] Li D, Wu Y, Kim P, Shi L, Yang P, Majumdar A. Thermal conductivity of individual silicon nanowires. Appl.Phys.Lett. 2003;83, 2934

[9] Park Y, Kim J, Kim H, Kim I, Lee K, Seo D, Choi H, Kim W. Thermal conductivity of VLS-grown rough Si nanowires with various surface roughnesses and diameters Appl. Phys. A 2011;104, 7

[10] Guthy C, Nam C, Fischer J.E. Unusually low thermal conductivity of gallium nitride nanowires. J. Appl. Phys. 2008;103, 064319

[11] Lee E. K, Yin L, Lee Y, Lee J. W, Lee S. J, Lee J. , Cha S. N, Whang D, Hwang G. S, Hippalgaonkar K, Majumdar A, Yu C, Choi B. L, Kim J. M, Kim K. Large thermoelectric figure of merits from SiGe nanowires by simultaneously measuring electrical and thermal transport properties. Nano Lett. 2012;12, 2918

[12] Westover T, Jones R, Huang J. Y, Wang G, Lai E, Talin A. A. Photoluminescence, Thermal Transport, and Breakdown in Joule-Heated GaN Nanowires. 


\section{ESTUDIO TEÓRICO DE LA DISTRIBUCIÓN DE TEMPERATURA Y SU EFECTO EN LA SEÑAL RAMAN DE NWS SEMICONDUCTORES BAJO UN HAZ LÁSER}

NanoLett. 2009;9, 257

[13] Ma D.D.D, Lee S.T, Shinar J. Strong polarization-dependent photoluminescence from silicon nanowire fibers. Appl. Phys. Lett. 2005;87, 033107

[14] Martelli F, Piccin M, Bais G, Jabeen F, Ambrosini S, Rubini S, Franciosi A. Photoluminescence of Mn-catalyzed GaAs nanowires grown by molecular beam epitaxy. Nanotechnol. 2007;18, 125603

[15] Demichel O, Oehler F, Calvo V, Noé P, Pauc N, Gentile P, Ferret P, Baron T, Magnea N. Photoluminescence of silicon nanowires obtained by epitaxial chemical vapor deposition. Physica E 2009;41, 963

[16] Xie X. Q, Liu W. F, Oh J. I, Shen W. Z. Optical absorption in c-Si/a-Si:H core/shell nanowire arrays for photovoltaic applications. Appl. Phys. Lett. 2011;99, 033107

[17] Cao L, White J. S, Park J. S, Schuller J. A, Clemens B. M, Brongersma M. L. Engineering light absorption in semiconductor nanowire devices. Nature mater. 2009;8, 643

[18] Brönstrup G, Jahr N, Leiterer C, Csaki A, Fritzsche W, Chistiansen S. Optical properties of individual silicon nanowires for photonic devices. ACS Nano 2010;4, 7113

[19] Scheel H, Reich S, Ferrari A. C, Cantoro M, Colli A, Thomsen C. Raman scattering on silicon nanowires: The thermal conductivity of the environment determines the optical phonon frequency. Appl. Phys. Lett. 2006;88, 233114

[20] Bhattacharya S, Samui S. Phonon confinement in oxide-coated silicon nanowires. Appl. Phys. Lett. 2004;84, 1564

[21] Gupta S. R, Xiong Q, Adu C. K, Kim U. J, Eklund P. C. Laser-induced Fano resonance scattering in silicon nanowires. Nano Lett. 2003;3, 627

[22] Adu K. W, Gutiérrez H. R, Kim U. J, Eklund P. C. Inhomogéneous laser heating and phonon confinement in silicon nanowires: A micro-Raman scattering study. Phys.Rev. B 2006;73, 155333

[23] Zhou G. W, Zhang Z, Yu D. P. Transmission electron microscopy study of Si nanowires. Appl. Phys. Lett. 1998;73, 677

[24] Torres A, Martín-Martín A, Martínez O, Prieto A. C, Hortelano V, Jiménez J, Rodríguez A, Sangrador J, Rodríguez T. Micro-Raman spectroscopy of Si nanowires: Influence of diameter and temperature. Appl. Phys. Lett 2010;96, 011904 
[25] Zhang L, Ding W, Yan Y, Qu J, Li B, Li Le-Yu, Yue K. T, Yu D. P. Variation of Raman feature on excitation wavelength in silicon nanowires. Appl. Phys. Lett. 2002;81, 4446

[26] Zardo I, Abstreiter G, Fontcuberta A. in Nanowires, ch.12, p.227.(ed. By P. Prete) (INTECH Croatia, 2010) (ISBN978-953-7619-79-4)

[27] Ahn Y, Dunning J, Park J. Scanning Photocurrent Imaging and Electronic Band Studies in Silicon Nanowire Field Effect Transistors. Nano Lett. 2005;5, 1367 [28] Chen H. Y, Chen R. S, Rajan N. K, Chang F. C, Chen L. C, Chen K. H, Yang Y. J, Reed M. A. Size dependent persistent photocurrent and surface band bending in m axial GaN nanowires. Phys. Rev. B 2001;84, 205443

[29] Jalilian R, Sumanasekera G. U, Chandrasekharan H, Sunkara M. K. Phonon confinement and laser heating effects in Germanium nanowires. Phys. Rev. B 2006;74, 155421

[30] Doerk G. S, Carraro C, Maboudian R. Temperature dependence of Raman spectra for individual silicon nanowires. Phys. Rev. B 2009;80, 073306

[31] Alarcón-Lladó E, Ibañez J, Cuscó R, Artús L, Prades J. D, Estradé S, Morante J. R. Ultraviolet Raman scattering in $\mathrm{ZnO}$ nanowires: quasimode mixing and temperature effects. J. Raman Spectrosc. 2011;42, 153

[32] Roodenko K, Goldthorpe I. A, MacIntyre P. C, Chabal Y. J. Modified phonon confinement model for Raman spectroscopy of nanostructured materials. Phys. Rev. B 2010;82, 115210

[33] Hsu I. K, Kumar R, Bushmaker A, Cronin S. B, Pettes M. T. Optical measurement of thermal transport in suspended carbon nanotubes. Appl. Phys. Lett. 2008;92, 063119

[34] Soini M, Zardo I, Uccelli E, Funk S, Koblmuller G, Fontcuberta A, Abstreiter G. Thermal conductivity of GaAs nanowires studied by micro-Raman spectroscopy combined with laser heating. Appl. Phys. Lett. 2010;97, 263107

[35] Doerk G. S, Carraro C, Maboudian R. Single Nanowire Thermal Conductivity Measurements by Raman Thermography. ACS Nano 2010;4, 4908

[36] Zhang Y, Cristofferson J, Shakouri A, Li D, Majumdar A, Wu Y, Fan R, Yang P. Characterization of heat transfer along a silicon nanowire using thermoreflectance technique. IEEE Trans. Nanotechnol. 2006;5, 67 


\section{ESTUDIO TEÓRICO DE LA DISTRIBUCIÓN DE TEMPERATURA Y SU EFECTO EN LA SEÑAL RAMAN DE NWS SEMICONDUCTORES BAJO UN HAZ LÁSER}

[37] Liu X. F, Wang R, Jiang Y. P, Zhang Q, Shan X. Y, Qiu X. H. Thermal conductivity measurement of individual CdS nanowires using microphotoluminescence. J. Appl. Phys. 2010;108, 054310

[38] Cao L, Fan P, Vasudev A. P, White J. S, Yu Z, Cai W, Schuller J. A, Fan S, Brongersma M. L. Semiconductor nanowire optical antenna solar absorbers. Nano Lett. 2010;10 439

[39] Cao L, Park J, Fan P, Clemens B, Brongersma M. L. Resonant germanium nanoantenna photodetector. Nano Lett. 2010;10, 1229

[40] Backhurst J. R, Harker J. H, Richardson J. F, Coulson J. M. Chemical Engineering ch.9. Vol 1, Sixth Edition (Butter worth -Heinemann, Oxford 1999).

[41] Sun B. K, Zhang X, Grigoropoulos C. P. Spectral optical functions of silicon in the range of 1.13-4.96 eV at elevated temperatures. Int. J. Heat Mass Transfer, 1997;40, 1591

[42] Kerker M. The scattering of light and other electromagnetic radiation; Ch.3 and Ch.8. (Academic Press, New York 1969).

[43] Kim H, Kim L, Choi H, Kim W. Thermal conductivities of Sil-xGex nanowires with different germanium concentrations and diameters. Appl. Phys. Lett. 2010;96, 233106

[44] Chen R, Hochbaum A. I, Murphy P, Moore J.E, Yang P, Majumdar A. Thermal Conductance of Thin Silicon Nanowires. Phys. Rev. Lett. 2008;101, 105501

[45] Wingert M. C, Chen Z. C. Y, Dechaumphai E, Moon J, Kim J, Xiang J, Chen R. Thermal Conductivity of Ge and Ge-Si Core-Shell Nanowires in the Phonon Confinement Regime. Nano Lett. 2011;11, 5507

[46] Lim J, Hippalgaonkar K, Andrews S. C, Majumdar A, Yang P. Quantifying Surface Roughness Effects on Phonon Transport in Silicon Nanowires. Nano Lett. 2012;12, 2475

[47] Kim H, Park Y, Kim I, Kim J, Choi H, Kim W. Effect of surface roughness on thermal conductivity of VLS-grown rough Sil-x Ge x nanowires. Appl. Phys. A 2011;104, 23

[48] Anaya J, Rodríguez T, Jiménez J. Nanowires, recent advances. (INTECH, ISBN980953-307-525-4, 2012 (to be published)).

[49] Duquesne J. Y. Thermal conductivity of semiconductor superlattices: Experimental study of interface scattering. Phys. Rev. B 2009;79, 153304 
[50] Banerjee R, Bhattacharya A, Ratan R, Shah A. P, Arora B. M, Genc A, Kar A. Root-like Structure at the Nanowire/substrate interface in GaAs Nanowires, Appl. Phys. Lett. 2006;88, 031919.

[51] Aspnes D. E, Studna A. A. Dielectric Functions and Optical Parameters of Si, Ge, GaP, GaAs, GaSb, InP, InAs, and InSb from 1.5 to $6.0 \mathrm{eV}$, Phys. Rev. B. 1983;27, 985.

[52] Zhang Y, Ram M. K, Stefenakos E. K, Goswami D, Y. Synthesis, Characterization and Application of ZnO Nanowires, Journal of Nanomaterials, 2012; DOI: 10.1155/2012/624520. [53] Raman C.V, The vibration spectra of crystals. Part I. Basic Theory. Proceedings of the Indian Academy of Sciences - Section A, 1947;26, 339

[54] Narayanaswamy P. K, Influence of temperature on the Raman spectra of crystals. Proceedings of the Indian Academy of Sciences - Section A, 1948;28, 40 [55] Spanier J. E, Robinson R. D, Zhang F, Siu-Wai C, Herman I. P, Size-dependent properties of CeO2-y nanoparticles as studied by Raman scattering. Phys.Rev. B. 2001;64, 245407

[56] Arora A. K, Rajalakshmi M, Ravindran T. R, Sivasubramanian V. Raman spectroscopy of optical phonon confinement in nanostructured materials. Journal of Raman Spectroscopy 2007;38, 60

[57] Wang R, Zhou G, Liu Y, Pan S, Zhang H, Yu D, Zhang Z. Raman spectral study of silicon nanowires: High-order scattering and phonon confinement effects. Phys. Rev. B 2000;61, 16827

[58] Belitsky V.I, Cantarero A, Cardona M, Trallero-Giner G, Pavlov S. Feynman diagrams and Fano interference in light scattering from doped semiconductors. J. Phys. Condens. Matter. 1997;9, 5965

[59] Doerk G.S, Carraro C, Maboudian R. Raman Spectroscopy for Nanomaterials Characterization. Ch. 17, Firsth Edition (edit. Kumar C. Springer - Heidelberg 2012). [60] Wua X, Yuc J, Rena T, Liua L. Micro-Raman spectroscopy measurement of stress in silicon. Microelectronics Journal 2007;38, 87

[61] Nilsson G, Nelin G. Study of the Homology between Silicon and Germanium by Thermal-Neutron Spectrometry. Phys. Rev. B. 1972;6, 3777

[62] Wu J.Polarized ligth scattering from individual semiconductor nanowires. Tésis, 


\section{ESTUDIO TEÓRICO DE LA DISTRIBUCIÓN DE TEMPERATURA Y SU EFECTO EN LA SEÑAL RAMAN DE NWS SEMICONDUCTORES BAJO UN HAZ LÁSER}

Universidad de Pensilvania, 2008. [63] Jiménez J, de Wolf I, Landesman J. P. Microprobe Characterization of Semiconductors; Optoelectronic properties of semiconductors and superlattices. Ch 2, Vol 17, ( ed. by J. Jiménez, Taylor and Francis, New York 2002)

[64] Burke H. H, Herman I. P. Temperature dependence of Raman scattering in Ge1-xSix alloys. Phys. Rev. B. 1993;48, 15016

[65] Balkanski M, Wallis R. F, Haro E. Anharmonic effects in light scattering due to optical phonons in silicon. Phys. Rev. B 1983;28, 1928 
The first principle is that you must not fool yourself - and you are the easiest person to fool.

Richard Feynman

\section{Espectroscopia Raman en NWs de Silicio y Silicio-Germanio}

\subsection{Introducción}

La espectroscopia Raman es una de las herramientas de caracterización más utilizadas en caracterización de semiconductoras, habiendo sido extensamente utilizada para la caracterización de NWs semiconductores $[1-7]$. En muchos de estos estudios,y con el objetivo de ganar en intensidad de señal, la adquisición del espectro Raman se realiza sobre muestras compuestas por múltiples NWs simultáneamente excitados [1,2,8 - 19]. Cuando se procede de esta manera, NWs de distintos diámetros, longitudes y características superficiales contribuyen simultáneamente a la señal Raman recolectada. En el capítulo anterior, se ha mostrado como la intensidad con la que contribuye cada NW simultáneamente excitado al espectro final es muy dependiente de las características intrínsecas de cada NW y de su posición en el área de iluminación. Típicamente, el espectro Raman de los NWs aparece ensanchado y desplazado frente al espectro Raman procedente del mismo material volúmico $[1,2,13,18,19]$. Estos efectos pueden ser atribuidos a la temperatura o 


\section{ESPECTROSCOPIA RAMAN EN NWS DE SILICIO Y SILICIO-GERMANIO}

al confinamiento fonónico, que a su vez dependen de las dimensiones de los NWs analizados y de sus condiciones de excitación. El confinamiento fonónico tiene el efecto de ensanchar el pico Raman, desplazarlo y asimetrizarlo hacia el lado de las bajas frecuencias $[1,20,21]$, pero no introduce un efecto relevante para NWs cuyos diámetros son mayores a $20 \mathrm{~nm}$, siendo su influencia cada vez mayor según se reduce el diámetro de los NWs [22]. Por otro lado, la temperatura tiene un efecto similar, ya que ensancha el pico y lo desplaza hacia bajas frecuencias, pero de forma simétrica y causando unos desplazamientos de pico Raman más acusados [22]. Estos efectos provocan que la interpretación del espectro Raman de los NWs semiconductores no sea un proceso exento de confusión, convirtiéndose dicho análisis en una tarea mucho más compleja que cuando se analiza el espectro Raman del mismo semiconductor volúmico.

Los NWs de silicio y silicio-germanio analizados en este capítulo han sido fabricados por el método VLS por varios colaboradores ajenos al grupo de semiconductores OptronLab de la universidad de Valladolid (ver capítulo 1), y con diferentes condiciones de crecimiento, teniendo en todo caso unos diámetros típicos entre 50 y $100 \mathrm{~nm}$, de tal modo que el efecto del confinamiento cuántico no tiene relevancia en la señal obtenida de ellos. El espectro Raman ha sido adquirido con un espectrómetro Labrham UV-HR 800 de Jovin Yvon como el descrito en el capítulo 1. Se han utilizado dos fuentes láser diferentes, una de $532.2 \mathrm{~nm}$ de longitud de onda, procedente de un láser Nd:YAG doblado, y otra procedente de un láser de $\mathrm{He}-\mathrm{Ne}$ con $632.8 \mathrm{~nm}$ de longitud de onda. El objetivo utilizado en ambos casos es un Olympus 100X con una apertura numérica de 0.95 , resultando en un diámetro para el área de iluminación efectiva de entre 0.9 y $1 \mu \mathrm{m}$ [23]. El equipo utilizado permite variar la potencia con la que se ilumina la muestra mediante un juego de filtros, permitiendo un rango de entre 5 y $500 \mu \mathrm{W}$ para el láser Nd:YAG y de entre 14 y $1300 \mu \mathrm{W}$ para el He-Ne, siendo estas potencias medidas en el plano focal.

En este capítulo, se resumen los resultados obtenidos en NWs de silicio y siliciogermanio de los que se ha realizado la caracterización Raman teniendo en cuenta la posición de los NWs en el área de iluminación, la longitud de onda del haz láser, las condiciones de disipación térmica, para muestras compuestas por varios NWs ó NWs individuales, mostrando claramente el efecto del calentamiento inducido por el láser que ilumina los NWs. Además de eliminar la confusión que rodea la 
caracterización Raman en NWs semiconductores, se muestra también una serie de resultados experimentales que no tienen equivalencia en la caracterización Raman de semiconductores volúmicos.

\subsection{Espectroscopia Raman en NWs de silicio.}

\subsubsection{Señal Raman procedente de conjuntos de NWs}

Un problema crucial cuando se realiza espectroscopia Raman en muestras de reducido volumen es la baja señal Raman debido al reducido volumen de scattering (ver ecuación 1.11). Una forma simple de solventar este problema cuando se trabaja con NWs consiste en realizar la medida en varios NWs simultáneamente, de este modo se aumenta mucho el volumen de scattering y, a priori, se consigue que la señal se intensifique, facilitando por tanto su análisis. Esta ha sido la manera de caracterizar NWs semiconductores mediante espectroscopia Raman escogida por diversos autores [1,2,8 - 19], y se ha justificado aduciendo a que pese a la dispersión en los diámetros de los NW simultáneamente probados, la señal Raman que procede de cada uno es independiente y además prácticamente idéntica. De este modo, la señal resultante es una señal más potente y que aporta la misma información que la obtenida en un único NW. El problema de esta metodología emerge cuando la señal de cada NW no es idéntica a la de sus vecinos simultáneamente excitados por el haz láser, como ya hemos puesto de manifiesto en capítulos anteriores. Cuando se realiza la caracterización Raman en múltiples NWs, típicamente se realiza sobre el mismo substrato en el que se han crecido los NWs [1, 2, 8 - 19]. En nuestro caso, estos substratos presentan una densidad de entre $5 \times 10^{6}$ y $2 \times 10^{6}$ NWs por centímetro cuadrado, lo que implica que bajo el área de iluminación efectiva del haz láser se pueden excitar como mucho algo más de una decena de NWs (ver figura 4.1). Al realizar un experimento de caracterización Raman en una muestra como la mostrada en la figura 1, en la que son excitados unos cuantos hilos simultáneamente, tenemos que tener en cuenta que la potencia del haz láser en el área de iluminación no es homogénea. En la sección 3.3 del capítulo anterior, mostramos teóricamente como las diferentes condiciones de excitación a las que 


\section{ESPECTROSCOPIA RAMAN EN NWS DE SILICIO Y SILICIO-GERMANIO}

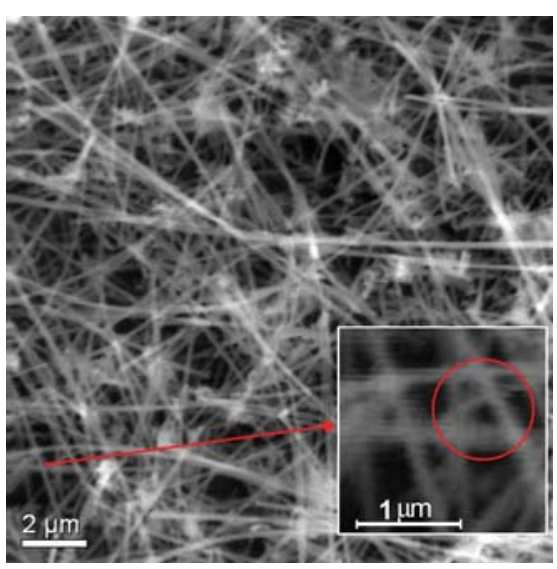

Figura 4.1: Imagen SEM de una muestra de NWs de silicio tomada sobre el substrato en el que han sido crecidos. En la ampliación se muestra la escala del área de iluminación efectivo del haz láser, puede verse como incluso en una muestra tan densamente poblada como esta, solo unos pocos NWs son iluminados simultáneamente

está sometido cada NW pueden deformar el espectro Raman, bien sea por temperatura o por efecto de confinamiento cuántico; incluso cuando se iluminan NWs del mismo diámetro, su redistribución dentro del área de iluminación modificaba la forma del espectro Raman obtenido. Este resultado teórico puede confirmarse experimentalmente en la figura 4.2, donde puede observarse como al ir barriendo la muestra en pasos de $0.5 \mu \mathrm{m}$, los espectros que se obtienen son muy diferentes. Puede observarse como los espectros obtenidos presentan un gran desplazamiento hacia bajas frecuencias y son mucho más anchos que el pico Raman característico del silicio volúmico. Además, los espectros son asimétricos. Típicamente, esta deformación podría atribuirse a la existencia de confinamiento cuántico y de calentamiento de los NWs. Sin embargo, en la muestra analizada los NWs tienen unos diámetros cercanos a los $100 \mathrm{~nm}$, y por tanto muy superiores al límite en el que el confinamiento fonónico tiene un efecto relevante en la forma de los NWs. Además, puede observarse como al barrer la muestra, llega a producirse incluso un desdoblamiento en dos picos, reduciéndose la asimétrica en los espectros al producirse dicha separación. Este comportamiento es el equivalente al mostrado en la sección 3.3 del capítulo 3 para NWs que son calentados por el haz láser de forma muy diferente; es decir, en el área iluminada del que procede la señal Raman coexisten NWs a muy diferente temperatura y que por tanto contribuyen de muy diferente 

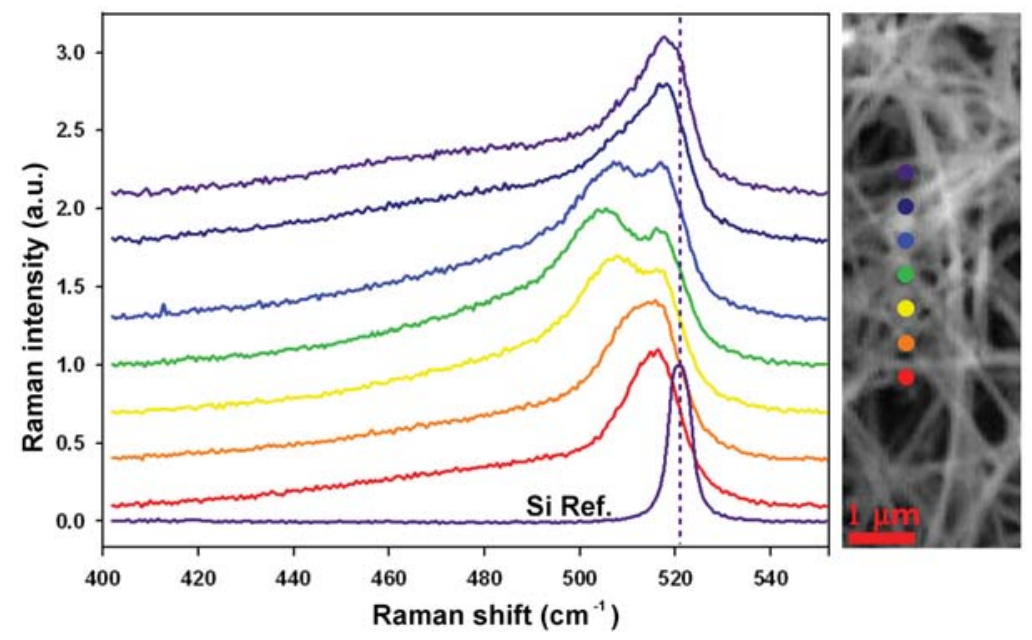

Figura 4.2: Espectros Raman obtenidos de una muestra donde son excitados múltiples NWs simultáneamente y el haz láser es enfocado en distintos puntos de la muestra siguiendo una linea con un paso de $0.5 \mu \mathrm{m}$. El haz láser utilizado es el He-Ne de 632.8 $\mathrm{nm}$ de longitud de onda y con una potencia de $1300 \mu \mathrm{W}$. Los puntos que representan donde está centrado el haz láser deben ser tomados como una guía para el ojo; conviene recordar que el punto donde se sitúa el haz láser se fija por microscopia óptica, y el alineamiento entre ambas técnicas no es perfecto. 


\section{ESPECTROSCOPIA RAMAN EN NWS DE SILICIO Y SILICIO-GERMANIO}

forma al espectro resultante. Este comportamiento es completamente compatible con los resultados teóricos mostrados en el capítulo 3 y pone de manifiesto como la coexistencia de distintos NWs bajo el haz láser puede introducir confusión en la interpretación de los espectros obtenidos. De hecho para explicar resultados similares a los mostrados en la figura 4.2, diversos autores han propuesto complejos modelos que recurren a hipótesis como son la existencia de inmensos gradientes de temperatura en la región iluminada de cada NW $(\delta T>600 \mathrm{~K} / \mu \mathrm{m})$ o a la iluminación simultanea de varios cientos de NWs [14 - 16], que a la vista de lo mostrado en la figura 4.1 y a los resultados teóricos de la sección 3.2.4 resultan del todo improbables.

\subsubsection{Influencia del medio en el que se encuentran los NWs}

La muestra sobre la que se ha obtenido el espectro Raman mostrado en la figura 4.2 puede considerarse completamente inmersa en aire, salvo por uno de los extremos donde se se produce el contacto de los NWs con el substrato. Este contacto puede ir desde un buen contacto térmico a una situación en la que alguno de los NWs iluminados esté seccionado de dicho substrato, i.e. sólo esté en contacto con otros NWs, pudiendo considerarse un NW aislado. Por tanto, las temperaturas que alcanza cada NW simultáneamente iluminado pueden diferir enormemente. La temperatura alcanzada por los NWs puede extraerse mediante la señal Raman de dos formas diferentes, bien mediante el cociente entre las intensidades Stokes /antiStokes [24, 25], o bien mediante la variación que se observa en la posición del pico Raman y en la anchura a media altura de dicho pico [2, 26, 27, 28]. La obtención de la temperatura por el cociente de intensidades Stokes/anti-Stokes tiene la dificultad añadida de que la intensidad anti-Stokes es muy débil salvo que la temperatura sea muy elevada, introduciendo cierta indeterminación en la medida. Además, en nuestro equipo resulta imposible obtener dicha intensidad anti-Stokes dado que para obtenerla haría falta un filtro de tipo Notch y el equipo utiliza un filtro pasabanda. El segundo método para estimar la temperatura encierra menos indeterminación y permite estimar de forma simple las temperaturas alcanzadas en la muestra. El desplazamiento del pico Raman a bajas frecuencias por efecto de la temperatura sigue 
un patrón lineal dado por [26]:

$$
\omega(T)=521( \pm 1,2)-0,022( \pm 0,001) \times(T-273,15)
$$

Mientras que la temperatura determinada a través de la anchura a media altura, $\Gamma(T)$, se puede obtener como [27]:

$$
\Gamma(T)=2,8+0,0102 \times(T-273,15)
$$

La determinación de la temperatura mediante la variación de la anchura a media altura es un proceso mucho más delicado que la determinación a través del pico Raman, ya que para determinar la anchura real del espectro Raman uno debe obtener el perfil de Voigt asociado [23, 28, 29], procedente de la deconvolución del pico Raman, de perfil Loretziano, y de la función de apertura experimental que presenta un perfil Gaussiano.

En la muestra de la figura 4.3-1 se presenta un espectro obtenido en la muestra de la 4.1 y con las mismas condiciones de excitación utilizadas para adquirir los espectros mostrados en 4.2. En este caso, se ha buscado una zona en la cual el espectro Raman no presenta la separación en dos picos tan representativa del calentamiento inhomogéneo de los distintos NWs iluminados. Puede observarse como el espectro obtenido es claramente asimétrico, lo que podría atribuirse al efecto del confinamiento fonónico. Por otro lado, si uno calcula las temperaturas mediante el desplazamiento de pico y mediante la anchura a media altura, se obtiene una gran discrepancia; la temperatura calculada por el desplazamiento de pico es de apenas $430 \mathrm{~K}$, mientras que la temperatura procedente de la anchura es cercana a los 1000K. Bajo nuestra interpretación teórica, esto se justifica mediante la existencia de NWs con distintos diámetros a unas temperaturas muy diferentes que contribuyen al espectro con diferente intensidad y forma. Para demostrar esto, podemos recurrir a otro resultado mostrado en el capítulo 3, donde se observaba como al introducir los NWs en un medio mucho más denso, como puede ser el agua, las temperaturas alcanzadas por los NWs disminuían drastícamente. En el espectro 2 de la figura 4.3 se muestra el efecto de depositar una gota de agua en la zona iluminada. Dada la pequeña cantidad de agua depositada, las condiciones de iluminación no varían sensiblemente, introduciéndose una potencia equivalente en los 


\section{ESPECTROSCOPIA RAMAN EN NWS DE SILICIO Y SILICIO-GERMANIO}

NWs iluminados. Puede observarse como el espectro 2, que se ha obtenido sobre los mismos NWs que el espectro 1, se asemeja mucho más al espectro obtenido del silicio de referencia, apareciendo simétrico y ligeramente desplazado a una menor frecuencia; demostrando por tanto, que la asimétria, el ensanchamiento y el gran desplazamiento de pico desaparecen al introducir los NWs en un medio en el que los NWs pueden disipar de una manera más eficiente la energía que el haz láser deposita en ellos.

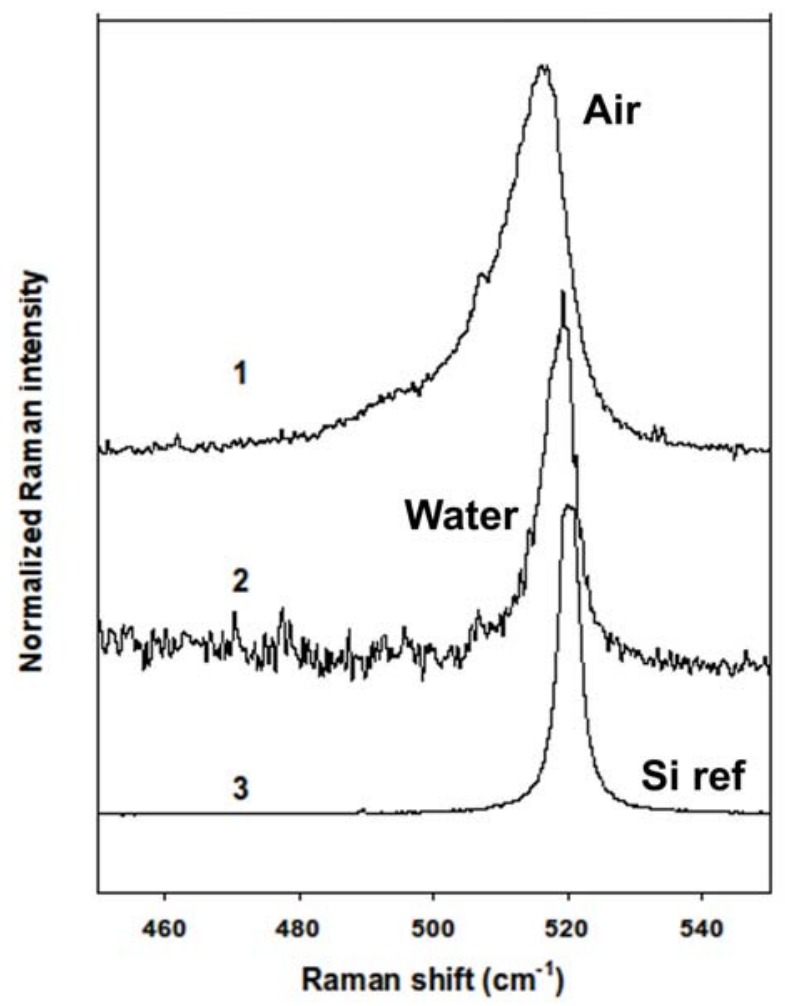

Figura 4.3: Espectros Raman tomados en la muestra de la figura 4.1 cuando está inmersa en aire (1) y en agua (2). Se muestra también el espectro Raman característico del silicio volúmico para comparar. La potencia utilizada es de $1300 \mu \mathrm{W}$ con el láser de He-Ne de $632.8 \mathrm{~nm}$ de longitud de onda 


\subsubsection{Análisis del espectro Raman procedente de un conjunto de NWs en función de la potencia del haz incidente}

En muestras donde se están iluminando decenas de NWs es muy difícil realizar un análisis detallado de los diferentes efectos que pueden contribuir al espectro Raman final que se obtiene de ellos. La dispersión en diámetros, longitudes y características superficiales de cada NW, unido a que los NWs no siempre están en el mismo plano focal, y que de estarlo ocupan posiciones muy diferentes en el área de iluminación, complican mucho la extracción de conclusiones claras de su espectro Raman. Por ello, en vez de analizar una muestra muy densa de NWs como la mostrada en la figura 4.1, se ha analizado la muestra mostrada en la figura 4.4A) compuesta por solo dos NWs. Para componer esta muestra se han separado los NWs de una muestra similar a la mostrada en la figura 4.1 mediante un raspado. El substrato sobre el que se han depositado es aluminio, de forma que el contacto térmico entre los NWs y el substrato sea bastante eficiente a la hora de disipar la energía depositada por el haz láser en los NWs. Los NWs son de silicio crecidos utilizando oro como catalizador y las bolas que se observan en la superficie del substrato en la figura 4.4-A) son contaminaciones de origen polimérico procedentes de a capsula en la que se conservan los NWs sumergidos en etanol y que no contribuyen a la señal Raman en el rango de interés. Los NWs analizados tienen diámetros de $\approx 50 \mathrm{~nm} \mathrm{y} \approx 80 \mathrm{~nm}$ respectivamente, están situados muy próximos y paralelos de forma que se eliminen efectos introducidos por la orientación de cada NW respecto al eje de polarización del haz láser incidente (ver capítulo 5, efectos de polarización en NWs bajo un haz linealmente polarizado). En esta muestra se ha recolectado el espectro Raman cuando el haz láser se centra en diferentes puntos siguiendo una linea que cruza transversalmente ambos NWs en pasos de $100 \mathrm{~nm}$. Este experimento es equivalente al análisis teórico realizado en el capítulo 3 sección 3.2.5, y más concretamente en la figura 3.13. La excitación se ha realizado con el láser de Nd:YAG de $532.8 \mathrm{~nm}$ de longitud de onda utilizando dos potencias, una de $500 \mu \mathrm{W}$ con la que se obtiene una buena señal pero que introduce un calentamiento nada despreciable en la muestra, y otra mucho más baja de $5 \mu \mathrm{W}$. Para obtener una señal perceptible con esta potencia tan baja, el tiempo de adquisición de cada espectro Raman ha sido cerca de 100 veces más largo que con la potencia de $500 \mu \mathrm{W}$, resultando en un experimento muy delicado de llevar a cabo debido a la dificultad 


\section{ESPECTROSCOPIA RAMAN EN NWS DE SILICIO Y SILICIO-GERMANIO}

de mantener las condiciones de medida durante periodos de tiempo mayores a 20 horas. Para identificar la zona sobre la que se ha realizado el corte transversal, primero se ha realizado un mapa con la potencia más alta y un paso de $100 \mathrm{~nm}$ x 200 $\mathrm{nm}$, de este modo puede situarse con un error relativamente pequeño la posición de la muestra (Este método se detalla un poco más adelante, en la figura 4.6). Una vez posicionada la muestra, se han realizado dos medidas sucesivas sobre la misma linea transversal representada en la figura 4.4-A), una para cada potencia de láser escogida, de modo que podamos asegurar que se está midiendo sobre la misma configuración en ambos casos. En la figura 4.4-B) podemos observar como moviendo el láser a través de la linea transversal a los dos NWs, los espectros obtenidos van modificando su forma, haciéndose más anchos y con un mayor desplazamiento de pico Raman cuanto más cerca del centro del spot láser están situados. Por otro lado, cuando el haz se aleja de los NWs la forma del espectro obtenido va haciéndose más estrecha y el pico se desplaza a mayores frecuencias. Incluso podemos observar como en 4 y 5 aparece levemente un doble pico que nos indica que unos de los NWs está a una temperatura mucho mayor que el otro. En la figura 4.4-C) podemos observar como al trabajar con una potencia tan baja, todos los espectros tienen la misma forma, con independencia de la posición que los NWs ocupan en el área de iluminación. Dado que ninguno de los NWs presenta efectos de confinamiento fonónico, esto nos demuestra claramente el origen exclusivamente térmico de la forma anómala observada en los espectros Raman adquiridos en muestras donde múltiples NWs son excitados. En la figura 4.4-D) se muestra el perfil de intensidades para ambas potencias, mostrando el mismo perfil gaussiano dado por el perfil de intensidades del haz láser. Este perfil gaussiano de intensidades está ensanchado sobre el tamaño real del spot láser por efecto de que la muestra ocupa una región de cerca de $150 \mathrm{~nm}$, y que aparece un acoplamiento electromagnético que modifica su comportamiento [30]. Esta configuración geométrica tan peculiar nos garantiza que al coincidir los perfiles de intensidad para ambas potencias, estos han sido adquiridos sobre los mismos puntos. Por último, en la figura 4.5 mostramos dos espectros adquiridos en la misma muestra para las dos potencias anteriormente referidas. En este caso hemos buscado el punto donde más claramente se puede apreciar la aparición de dos picos Raman diferenciados al utilizar la potencia más alta. El efecto de la temperatura puede observarse claramente en los picos correspondientes a los 

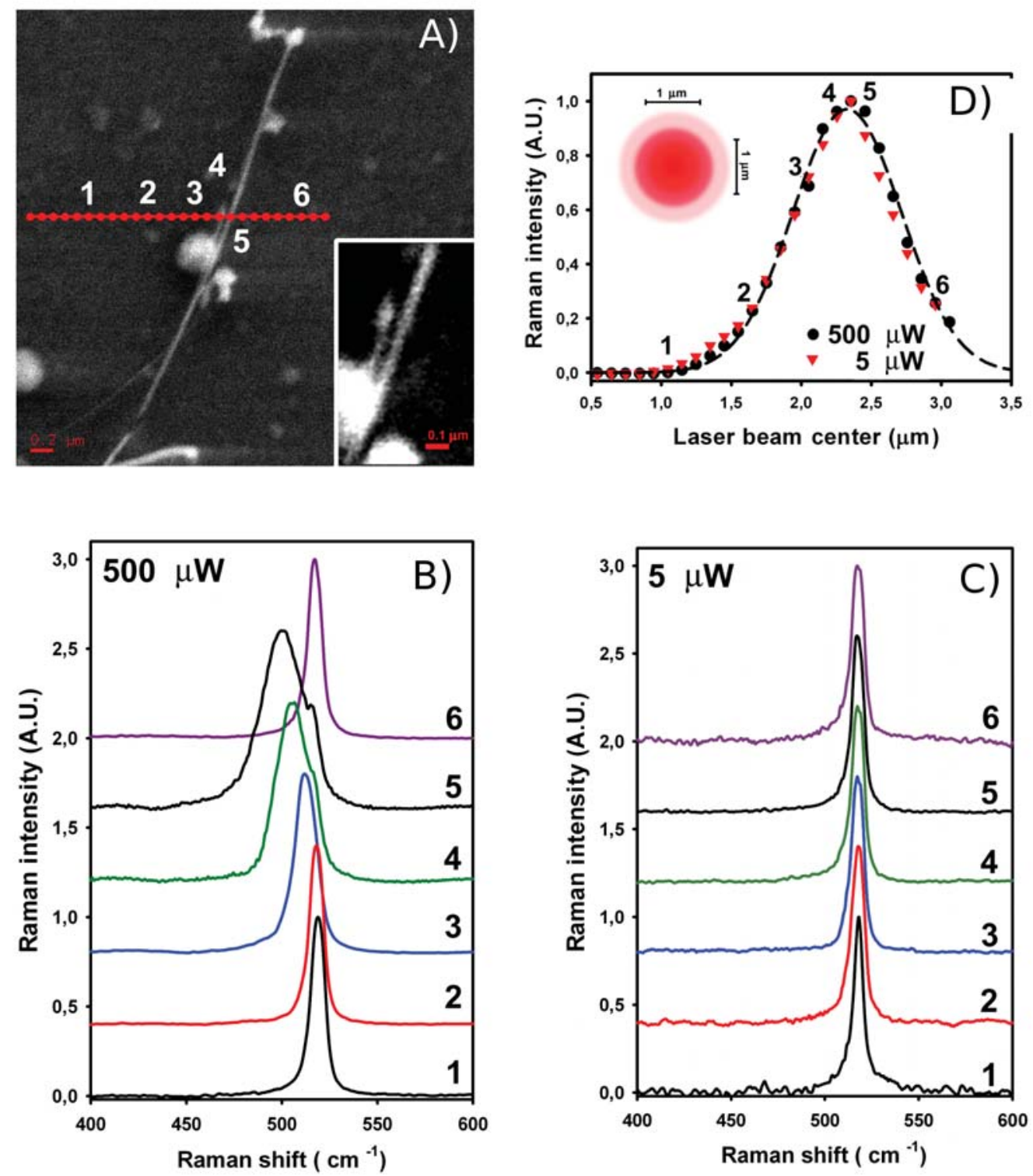

Figura 4.4: A) Imagen SEM de dos NWs de silicio paralelos, la linea de puntos corresponde a una guía para ojo que representa el movimiento de láser. Los números representan la posición del láser para los espectros mostrados posteriormente. B) Espectros Raman obtenidos al desplazar el láser a lo largo de la linea transversal mostrada en A) utilizando el láser de Nd:YAG de $532.8 \mathrm{~nm}$ de longitud de onda y 500 $\mu \mathrm{W}$ de potencia. C) Espectros Raman adquiridos en las mismas posiciones que en $\mathrm{A}$ ) con el láser a una potencia de $5 \mu \mathrm{W}$. D) Intensidad Raman normalizada obtenida en cada punto de la linea mostrada en A) para ambas potencias de láser utilizadas. Nótese como tienen un comportamiento gaussiano dado por la distribución de intensidad del haz láser y como al normalizarse los puntos se superponen perfectamente para las dos potencias utilizadas, mostrando que los espectros han sido adquiridos sobre la misma configuración espacial de la muestra. 


\section{ESPECTROSCOPIA RAMAN EN NWS DE SILICIO Y SILICIO-GERMANIO}

segundos ordenes del silicio y que aparecer entorno a $300 \mathrm{~cm}^{-1}$ y $900 \mathrm{~cm}^{-1}$. Cuando se compara con el espectro adquirido a baja potencia, puede observarse como el doble pico desaparece y los segundos ordenes recuperan su aspecto normal cuando los NWs no están siendo ultra-calentados.

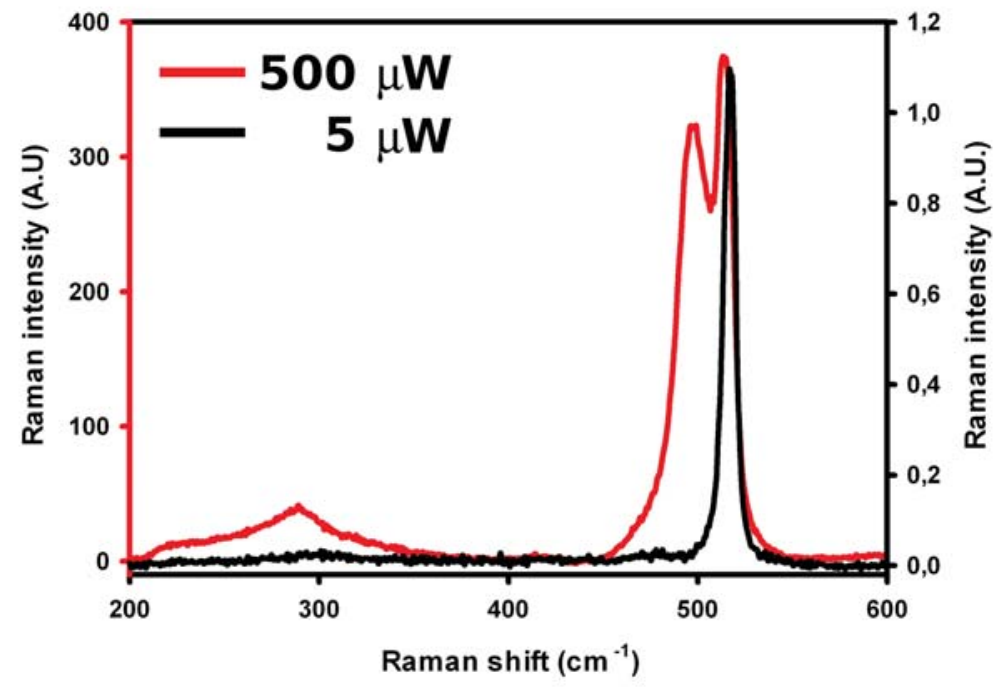

Figura 4.5: Espectros Raman adquiridos en la muestra que aparece en la figura 4.4-A) y en el mismo punto para ambas potencias. Puede observarse como para la potencia más elevada aparece una separación en dos picos del espectro Raman y como los segundos ordenes se deforman ostensiblemente por efecto de la temperatura. Cuando se utiliza la potencia más baja puede observarse como ninguno de estos efectos aparece, obteniéndose un espectro prácticamente idéntico al del silicio volúmico.

\subsubsection{Señal Raman procedente de NWs individuales}

En la sección anterior se ha mostrado como el análisis de la señal Raman procedente de conjuntos de NWs de silicio encierra un alto grado de incertidumbre debido a la diferente contribución de cada NW al espectro final. Incluso cuando la muestra tiene una configuración tan simple como la mostrada en 4.4-A), y los NWs están depositados en un substrato con buenas cualidades de conducción térmica, es necesario bajar mucho la potencia del haz láser para obtener una información más clara de los NWs. Esto implica alargar los tiempos de medida enormemente, resultando en una técnica experimental muy delicada y poco eficiente en escala temporal. Por 
lo tanto, la técnica de caracterización Raman de NWs semiconductores basada en la excitación simultáneamente de múltiples NWs no parece la más adecuada, siendo necesario caracterizar los NWs individualmente.

Cuando caracterizamos NWs individualmente, uno de los primeros problemas que es necesario resolver para realizar un correcto análisis del espectro Raman es determinar perfectamente su posición con respecto al haz láser incidente. Esto resulta complicado a priori, dado que dos de las dimensiones de los NWs están muy por debajo del límite de difracción que caracteriza el poder de resolución del microscopio confocal acoplado al espectrómetro Raman. Esto puede observarse al comparar las imágenes de un NW obtenidas mediante microscopia electrónica y la correspondiente imagen obtenida por microscopia óptica del mismo NW en la figura 4.6-A) y 4.6-B). Mediante la imagen SEM podemos atribuir unas dimensiones para el NW de $90 \mathrm{~nm}$ en diámetro y cerca de 6 micras en longitud, mientras que la imagen obtenida con el microscopio óptico, sólo nos permite dilucidar su geometría (ver 4.6-A) esquina superior con fondo rosa), y una vez ampliada y tratada la imagen nos muestra como el diámetro del NW está sobredimensionado en un orden de magnitud con respecto a sus dimensiones reales. Sin embargo, a través de los mapas de intensidad Raman y de la distribución gaussiana de intensidad del haz láser, puede determinarse perfectamente la localización del NW dentro del área de iluminación. En la figura 4.6- C) se muestran dos mapas de intensidades Raman realizadas a distinta potencia sobre el mismo NW, puede observarse como los mapas dibujan los mismos perfiles y como los máximos de intensidad se obtienen en los mismo puntos en ambos mapas, ubicando la posición en la que el NW está situado en el centro del spot láser.

\subsubsection{Señal Raman procedente de un NW inmerso en aire}

Una vez que tenemos establecida una forma de identificar la posición del NW, y que trabajamos sobre un único NW de silicio, es posible realizar un análisis de la señal Raman obtenida atendiendo a los diversos factores que pueden afectar a la señal, comenzando en esta subsección por analizar el efecto que tiene la posición que ocupa el NW en el área de iluminación cuando el NW no puede disipar de forma eficiente la energía depositada por el haz láser que lo ilumina.

En la figura 4.7-A) se muestra un NW de silicio de $\sim 100 \mathrm{~nm}$ de diámetro y varias 


\section{ESPECTROSCOPIA RAMAN EN NWS DE SILICIO Y SILICIO-GERMANIO}

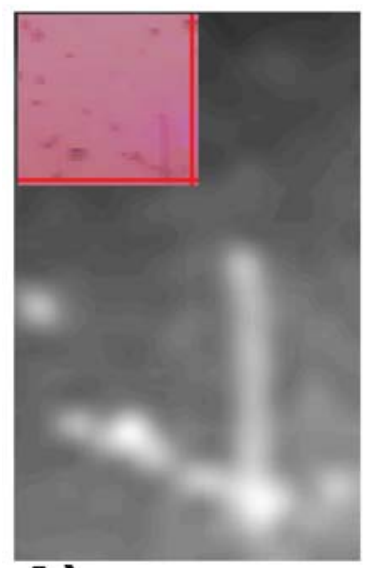

A)

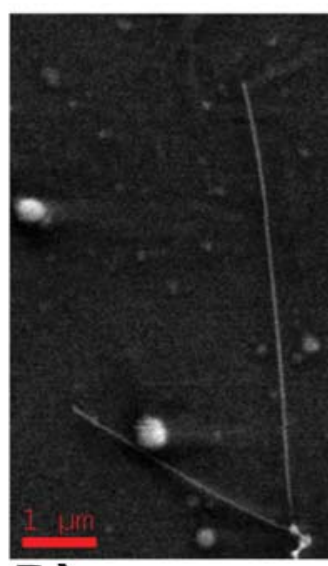

B)

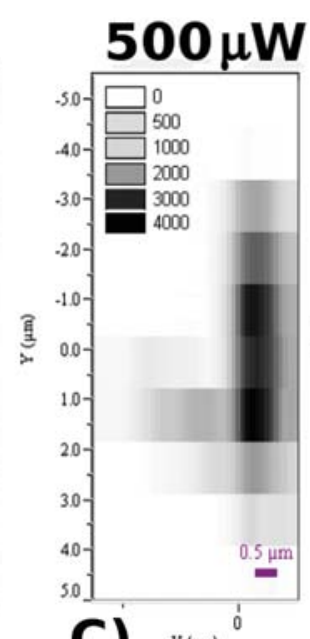

C)

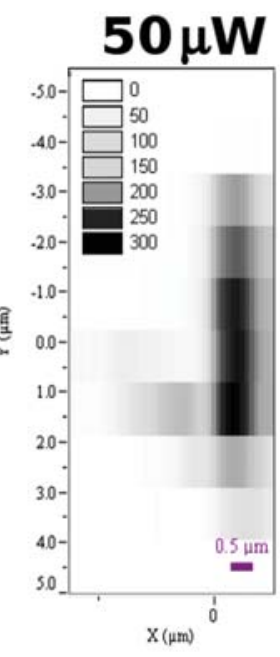

Figura 4.6: A) Arriba a la izquierda y con fondo rosa, se muestra la imagen obtenida mediante microscopia óptica confocal y un objetivo 100X de la zona donde se encuentra el NW. En escala de grises se muestra la misma imagen tratada y ampliada. B) Imagen SEM del mismo NW, puede observarse como los perfiles coinciden, si bien las dimension radial estimada de la imagen SEM es muy diferente de lo observado en A). C) Mapas de intensidad Raman obtenidos para el mismo NW utilizando dos potencias diferentes con el láser de Nd:YAG y $532.8 \mathrm{~nm}$ de longitud de onda. Puede observarse como en ambos casos se dibuja el mismo perfil, apareciendo los máximos de intensidad en la misma zona, identificando el punto donde el NW esta centrado en el spot láser gaussiano. 
decenas de micras en longitud, crecido por el método VLS utilizando oro como catalizador. El NW pertenece a una muestra donde los NWs están anclados al substrato donde han crecido, sin embargo el NW analizado se encuentra en otro plano focal diferente a los NWs que emergen anclados del substrato, y suficientemente separado de cualquier otro NW como para poder analizar su espectro de forma independiente. La unión con el substrato no es limpia, produciéndose en una bola de silicio y no directamente al substrato. Esto causa que la unión sea una barrera térmica que hace que el NW pueda considerarse como aislado del substrato y por tanto prácticamente inmerso en aire en su totalidad. El NW se ilumina con el láser de He-Ne de $632.8 \mathrm{~nm}$ y una potencia de $1300 \mathrm{muW}$. Con esta potencia y longitud de onda, el modelo teórico presentado en el capítulo 3 predice unas temperaturas de entre 900 y $1000 \mathrm{~K}$ en un NW de este diámetro, aunque la temperatura alcanzada dependerá de la conductividad térmica del NW y de su longitud. Con estas temperaturas, el espectro Raman adquirido debe aparecer muy ensanchado y desplazado hacia valores de frecuencia en más de $15 \mathrm{~cm}^{-1}$ según la ecuación 4.1 .

Cuando sobre este NW se realiza una serie de medidas en las que el haz láser se desplaza de forma transversal al NW (ver línea en la ampliación de la figura 4.7-A), puede observarse como la forma del espectro se modifica al cambiar la posición relativa del NW respecto del centro del haz láser (Figura 4.7-B)). Dado que no existen otros NWs cerca, este efecto se debe exclusivamente al NW iluminado, correspondiéndose bien con lo esperado para un NW cuya temperatura se incrementa en cada paso y posteriormente vuelve a la temperatura ambiente. En la figura 4.7C) podemos observar el espectro obtenido en la zona de máximo desplazamiento y ensanchamiento de pico, y su comparación con el espectro obtenido para el silicio volúmico. El espectro del NW presenta un gran desplazamiento de pico Raman a bajas frecuencias y un ensanchamiento del mismo, pero como puede observarse en la figura, se conserva la simetría del pico. Esto se corresponde perfectamente con lo esperado para una NW cuya temperatura se ha elevado considerablemente frente a la temperatura ambiente. En la figura 4.8-A) podemos observar la variación de la posición del pico Raman de los espectros mostrados en la figura 4.7-B) en función de la posición relativa del NW en el área iluminada por el haz láser. El desplazamiento hacia bajas frecuencias es muy brusco cuando el NW ocupa posiciones más próximas al centro del spot láser. Cuando el centro del haz láser se aleja del NW, la 


\section{ESPECTROSCOPIA RAMAN EN NWS DE SILICIO Y SILICIO-GERMANIO}
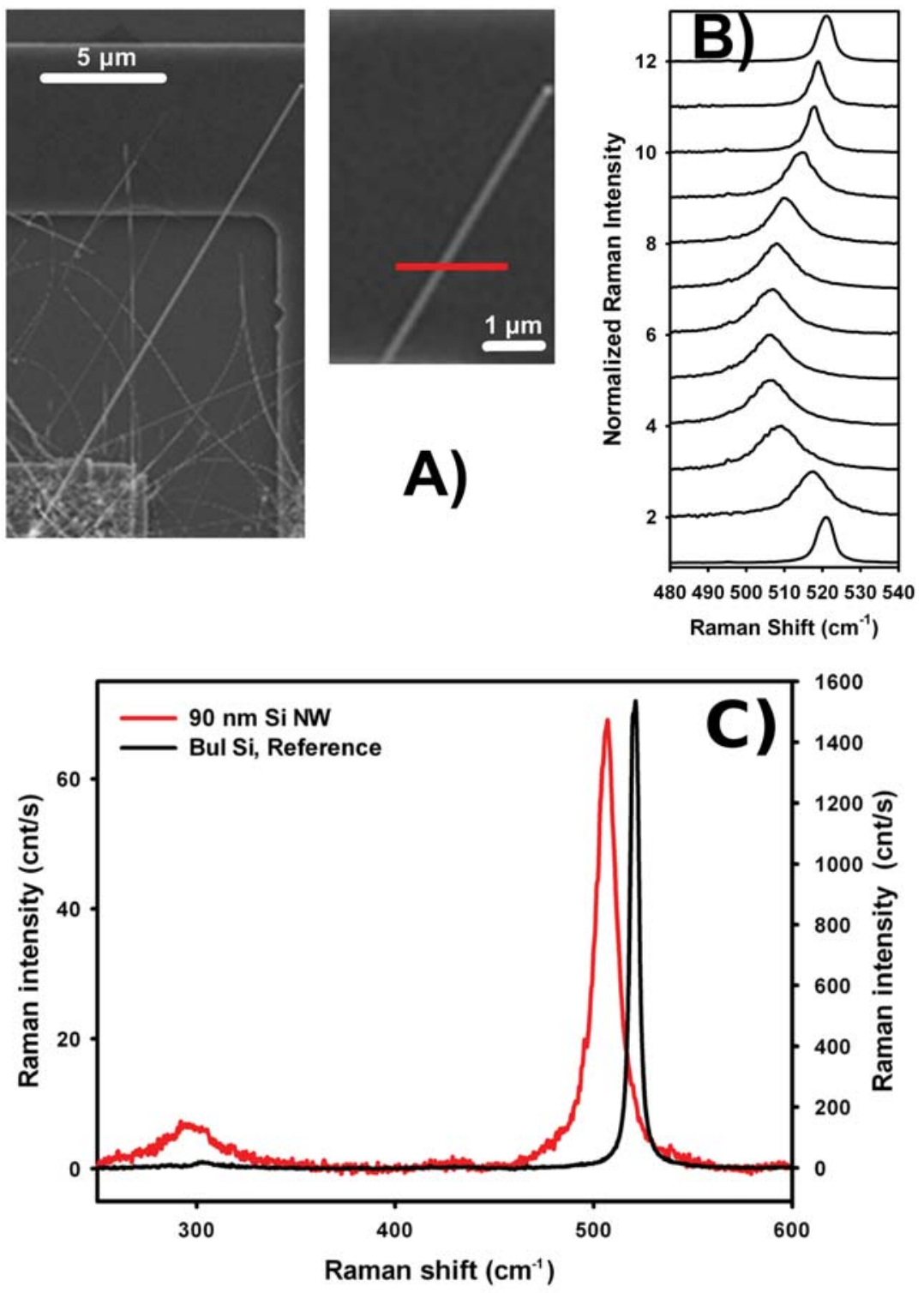

Figura 4.7: A) Imagen SEM del NW de silicio analizado y zona ampliada sobre la que se ha adquirido el espectro. Puede observarse como el NW esta completamente aislado. B) Serie de espectros tomados al variar el haz láser a los largo de la linea transversal que aparecer en a figura A) atravesando el NW. C) Comparación entre uno de los espectros en la zona de máxima intensidad de señal y el espectro correspondiente a al silicio volúmico. Observese como el pico Raman es simétrico pese al desplazamiento y ensanchamiento del pico frente al espectro correspondiente al silicio volúmico. 
posición del pico Raman se desplaza más lentamente hacia frecuencias más altas. Utilizando la ecuación 4.1 para obtener la temperatura del NW a través de la posición del pico Raman, se obtiene el resultado mostrado en la figura 4.8-B). Puede observarse como la temperatura calculada sigue un perfil gaussiano equivalente al calculado teóricamente en la figura 3.12-A) del capítulo 3, donde la anchura del spot láser es $\sim 0,9 \mu \mathrm{m}$, en perfecto acuerdo con lo calculado en la referencia [23]. Por otro lado las temperaturas alcanzadas son acordes a lo calculado mediante el modelo FEM descrito en el capitulo 3 para este diámetro de NW y características del láser que lo ilumina. Finalmente, cabe destacar que el ajuste a la curva de temperatura teórica es muy bueno en la parte izquierda de la figura 4.8-B), desviándose en la parte derecha. En la parte izquierda de la curva, el NW se calienta progresivamente al ser iluminado por una mayor potencia del haz láser, mientras que en la parte derecha, el NW cada vez es iluminado por una menor potencia. Dado que las medidas se hacen de forma consecutiva, i.e. el NW está continuamente iluminado por el haz láser, ambas partes sólo pueden ser idénticas si la velocidad a la que el NW se calienta es igual a la velocidad a la que el NW disipa la temperatura al medio que lo rodea. Como el medio que rodea al NW es aire, y sabemos que la disipación no es eficiente, el NW guarda parte del calor procedente de la medida anterior, y por tanto la desviación en la parte derecha muestra el efecto de la inercia térmica del NW.

\subsubsection{Espectro Raman procedente de un NW con buena disipación térmica en función de la potencia y longitud de onda del haz láser incidente}

Las condiciones de disipación son fundamentales para minimizar la temperatura inducida en los NWs al ser iluminados por el haz láser. En esta sección, se ha depositado un NW individual sobre un substrato de aluminio, de modo que si el contacto entre NW y substrato es bueno, la temperatura alcanzada por el NW va a ser mucho menor que cuando el NW se encuentra completamente rodeado por aire. El NW de silicio analizado ha sido crecido mediante el método VLS utilizando una aleación de oro-galio al $50 \%$ como catalizador, tiene un diámetro de $\sim 65 \mathrm{~nm}$ y cerca de 6 $\mu \mathrm{m}$ de longitud. Sobre este NW se ha analizado el efecto de la longitud de onda del haz incidente en el espectro Raman, y como afecta la variación de la potencia del haz al espectro obtenido. Las longitudes de onda utilizadas son $632.8 \mathrm{~nm}$ y 532.8 


\section{ESPECTROSCOPIA RAMAN EN NWS DE SILICIO Y SILICIO-GERMANIO}
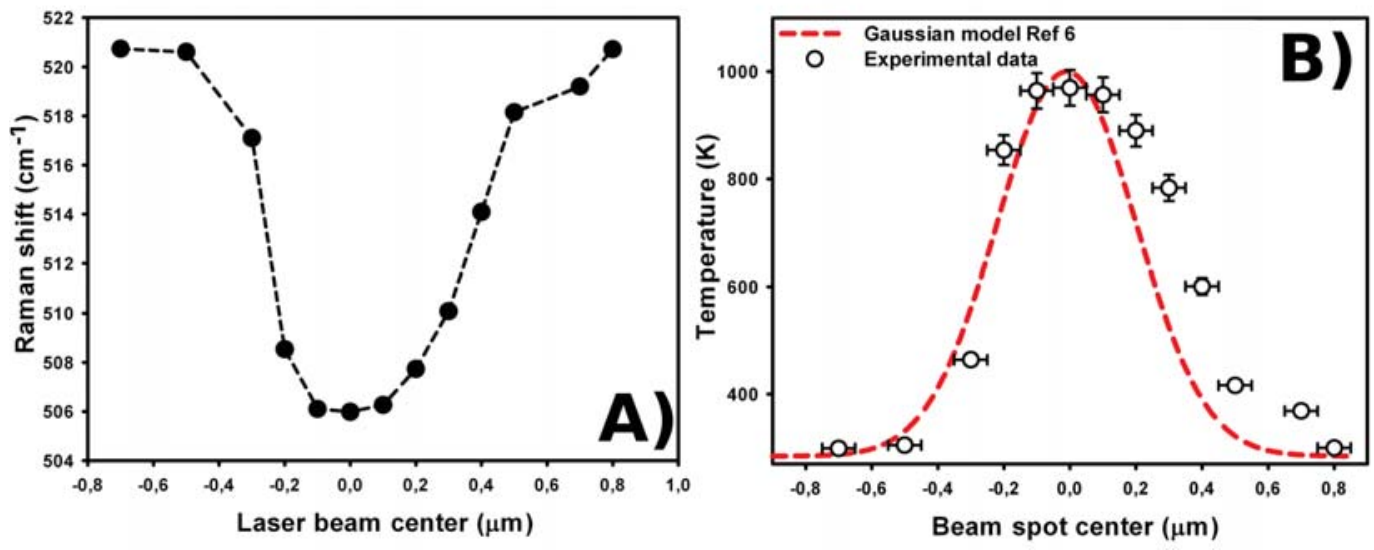

Figura 4.8: A) Perfil de posición del pico Raman en función de la posición que ocupa el NW mostrado en la figura 4.7-A) en el área iluminada por el haz láser. B) Perfil de temperaturas alcanzadas por el NW en función de la posición que ocupa en el área iluminada. Nótese el efecto de la inercia térmica del NW debido al ineficiente intercambio de energía con el aire que lo rodea en la desviación del perfil gaussiano observada en la parte derecha de la figura.

nm,, la potencia utilizada corresponde a $1300 \mu \mathrm{W}$ para el láser rojo, mientras que para el láser verde se han utilizado potencias de 5, 50 y $500 \mu \mathrm{W}$. De los cálculos realizados en en capítulo 3, sabemos que los NWs de silicio absorben mucha más energía del láser verde que del rojo, por tanto es esperable que si el contacto térmico entre el NW y el substrato no es perfecto, los espectros adquiridos con el láser verde a máxima potencia reflejen una mayor temperatura que los adquiridos con el láser rojo.

Para garantizar que para una potencia determinada del láser no pueden inducirse temperaturas suficientes en el NW para que el espectro Raman aparezca deformado, es necesario realizar un barrido transversal cruzando el NW. Cuando independientemente de la posición relativa que ocupa el NW en el área de iluminación, el espectro Raman obtenido tiene la misma forma, se garantiza que no hay efectos de temperatura para esa potencia. En la figura 4.9-B) y 4.9-C) se muestran los espectros obtenidos para distintas potencias y longitudes de onda en cuatro posiciones distintas del NW en el área de iluminación. Estas medidas están separadas en pasos de $0.2 \mu \mathrm{m}$, y van desde que el haz apenas ilumina al NW, al punto de máxima intensidad en el que el NW está prácticamente centrado en el área de iluminación. En 
la figura 4.9-B) se puede observar como a pesar de existir un buen contacto térmico entre el substrato y el NW, para una potencia de $500 \mu \mathrm{W}$ aparece un desplazamiento del pico Raman a bajas frecuencias que no se observa cuando se baja la potencia hasta $5 \mu \mathrm{W}$. En la figura 4.9-B), se muestran los espectros obtenidos con el láser de $632.8 \mathrm{~nm}$, siendo todos ellos independientes de la posición del haz con respecto al NW; dado que el láser rojo es mucho más potente que el verde, y no se observa un aumento de la temperatura en el NW, se confirma lo obtenido teóricamente a través del modelo FEM del capítulo 2. Este resultado revela el buen contacto térmico que existe entre el substrato y el NW, dado que con esta misma configuración para el láser y para un NW de un diámetro no muy distinto rodeado por aire, la temperatura escalaba hasta casi 1000K (ver figura 4.8-B) ). Las posiciones del pico Raman en función de la posición del haz láser respecto al NW en pasos de $100 \mathrm{~nm}$ se muestran en la figura 4.10-A) para todas las potencias y longitudes de onda. Cuando la potencia es suficientemente baja la posición del pico Raman es prácticamente constante a lo largo de todo el corte transversal y por tanto independiente de la posición relativa del NW con respecto al haz láser. Sin embargo, para el láser de Nd:YAG y una potencia de $500 \mu \mathrm{W}$ se aprecia como aparece un desplazamiento hacia bajas frecuencias a medida que el NW se aproxima al centro del spot láser. En la figura 4.10-B) se muestra las temperaturas alcanzadas en cada caso utilizando la ecuación 4.1. El perfil de temperaturas para el láser verde más potente se ajusta perfectamente al perfil gaussiano obtenido en el capítulo 3, apareciendo además el mismo comportamiento observado en la figura 4.8-B) para el NW aislado. Si bien la escala de temperaturas es menor, se aprecia perfectamente el efecto de la inercia térmica del NW en la asimetría de la parte derecha de la curva de temperaturas. Cuando se baja la potencia, puede observarse como la curva de temperatura se aplana hasta hacerse prácticamente independiente de la posición que ocupa el NW en el área iluminada para $5 \mu \mathrm{W}$ en el láser verde, observándose un comportamiento similar cuando se ilumina con el láser rojo. Que las curvas mostradas en 4.10-A) y B) no presenten variaciones implica que los espectros obtenidos no están afectados por aumentos de temperatura, y dado que los NWs tienen unos diámetros por encima del confinamiento fonónico, la forma del espectro tampoco está afectada por estos efectos. Esto se confirma además por la forma simétrica del pico Raman. Por tanto los espectros obtenidos en estas condiciones son los espectros Raman propios del 


\section{ESPECTROSCOPIA RAMAN EN NWS DE SILICIO Y SILICIO-GERMANIO}
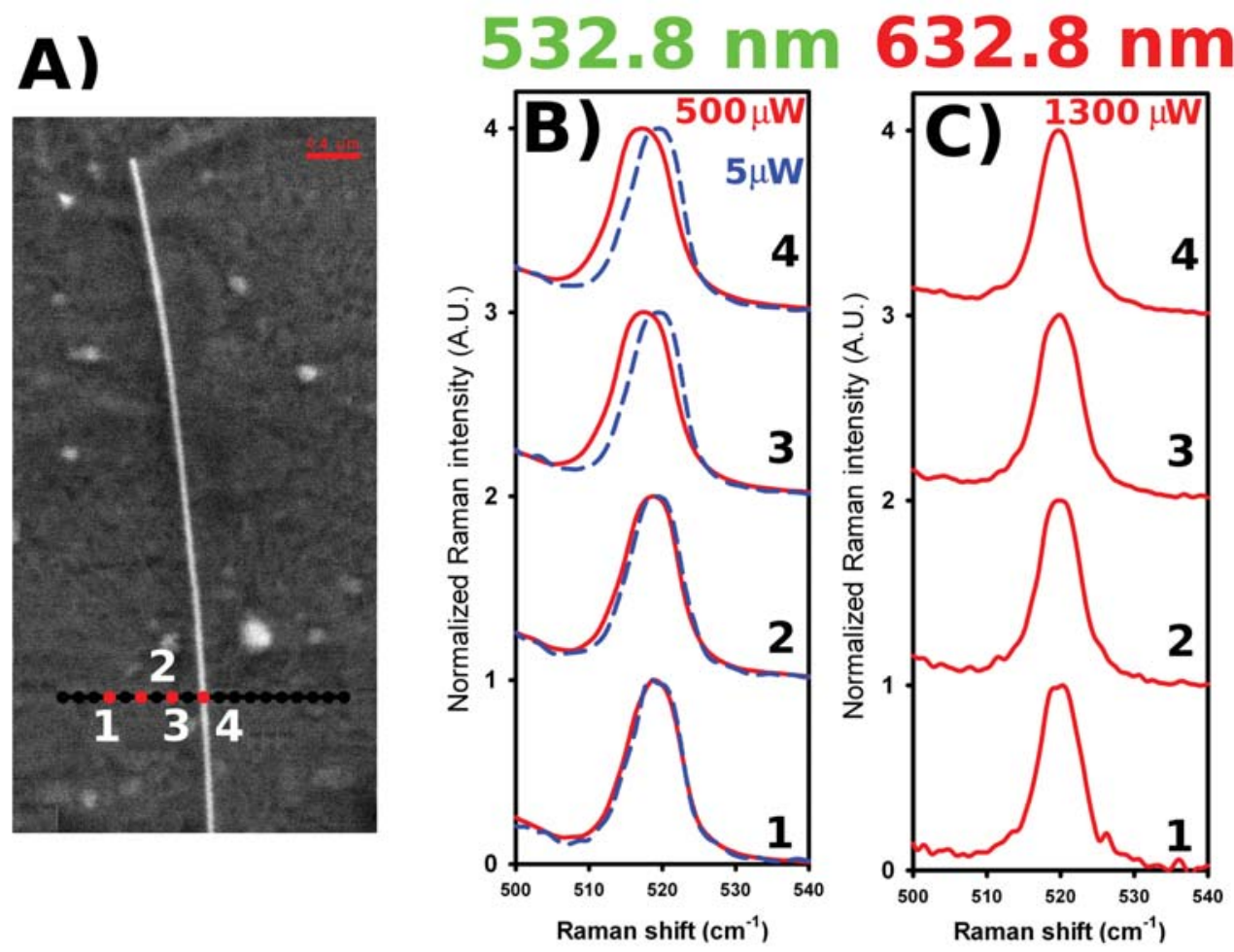

Figura 4.9: Imagen SEM de un NW de silicio depositado sobre un substrato de aluminio. La línea de puntos representa donde se ha centrado el haz láser durante el barrido transversal. B) Espectros obtenidos en los puntos numerados en A) utilizando el láser de Ne:YAG para $500 \mu \mathrm{W}$ y $5 \mu \mathrm{W}$. Puede observarse como para baja potencia los espectros son independientes de la posición de NW dentro del área de iluminación, mientras que para la potencia más alta se observa un desplazamiento hacia bajas frecuencias y un ensanchamiento por efecto de la temperatura alcanzada en el NW. C) Serie de espectros tomados en los mismos que en B) pero utilizando un láser de He-Ne con una potencia de $1300 \mu \mathrm{W}$. Pude observarse como los espectros son independientes de la posición relativa del NW dentro del área de iluminación, evidenciando que el NW no se está calentando apreciablemente. 

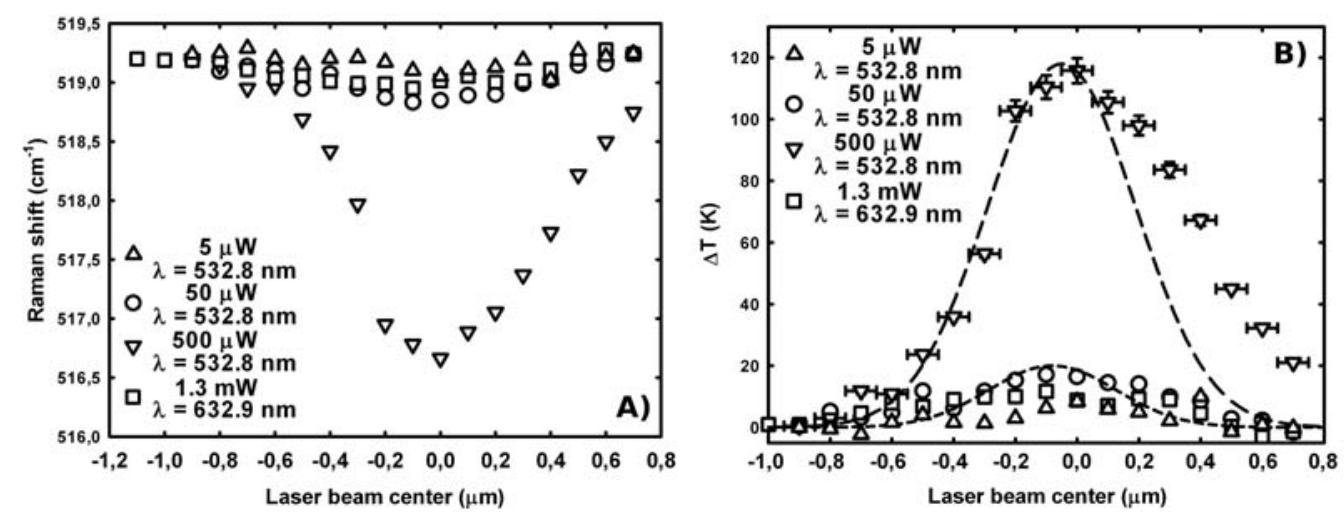

Figura 4.10: A) Posición del pico Raman en función de la posición del NW respecto al centro del spot láser para diferentes potencias y longitudes de onda. Puede observarse como al bajar la potencia, la posición del pico Raman se va haciendo independiente de la posición que ocupa el NW en el área de iluminación. B) Perfil de temperaturas obtenido de la figura A). La temperatura inducida cuando se utiliza el láser verde más potente se ajusta perfectamente en la parte izquierda a una distribución gaussiana como la predicha en el capítulo 3, mientras que en la parte derecha se puede apreciar el efecto de la inercia térmica mostrado para otros NWs. Nótese la gran diferencia existente al variar la longitud de onda del láser, no apreciándose incremento de temperatura alguno para el láser rojo incluso cuando su potencia nominal es superior a la del láser verde. 


\section{ESPECTROSCOPIA RAMAN EN NWS DE SILICIO Y SILICIO-GERMANIO}

NW de silicio. Estos son muy similares a los obtenidos del silicio de referencia, pero en todos los casos analizados la posición del pico Raman aparece desplazada hacia bajas frecuencias cerca de $1 \mathrm{~cm}^{-1}$. Este desplazamiento no se debe a temperatura como ya hemos demostrado, y dado que los picos son simétricos tampoco se debe al efecto de confinamiento. Esta discrepancia entre la posición del pico Raman para el material volúmico y el material nanoestructurado ha sido observada por otros autores [26], obteniendo unos desplazamientos similares a los aquí observados. Una posible explicación a este efecto puede encontrarse en la referencia [31], donde se muestra como el parámetro de red en los NWs de silicio no tiene por qué ser igual al parámetro de red del silicio volúmico. De hecho, en esta referencia se muestra como el parámetro de red es ligeramente mayor para los NWs de silicio, aumentando cuanto menor es el diámetro del NW. Un mayor parámetro de red implica una menor energía de vibración y por tanto un desplazamiento hacia bajas frecuencias del pico Raman característico [32,33]. Por tanto, esta pequeña variación de la posición del pico Raman en los NWs puede encontrar explicación bajo esta hipótesis. Dado que esta pequeña variación del parámetro de red es dependiente del diámetro de los NWs, dicha hipótesis podría confirmarse mediante un análisis del espectro Raman en función del diámetro de los NWs, eliminando por completo el efecto de temperatura. Un estudio de este tipo requiere disponer de NWs de diámetro controlado entre $25 \mathrm{~nm}$ y algunas centenas de $\mathrm{nm}$, de modo que pueda analizarse la posición del pico Raman de forma sistemática. Lamentablemente, las muestras disponibles en el momento de realizar esta investigación no han permitido un experimento de este tipo.

\subsubsection{Anomalías observadas en los espectros Raman obtenidos de NWs de silicio}

Al ampliar el rango espectral para el NW mostrado en la figura 4.9 de la sección anterior, puede observarse como los espectros mostrados en las figura 4.11-A) y 4.11-B), incorporan ciertas anomalías en comparación con el espectro Raman de silicio volúmico, las cuales requieren ser analizadas. La más evidente es la aparición de un pico adyacente al pico Raman del silicio, pero también resulta llamativa 
la enorme intensidad en la señal Raman procedente del NW. Estos dos efectos anómalos son el foco de interés de esta sección.

\subsubsection{Pico adyacente a $495 \mathrm{~cm}^{-1}$}

La aparición de un segundo pico adyacente al pico Raman característico del silicio, situado entorno a $495 \mathrm{~cm}^{-1}$, puede resultar a priori similar a lo ya observado en la figura figura 4.5 cuando se iluminaban varios NWs y eran inhomogéneamente calentados . Sin embargo, en este caso sabemos que estamos iluminando un NW individual (Ver imagen SEM en 4.9-A) ), por lo que la procedencia de este pico no puede deberse a la existencia de contribuciones pertenecientes a otros NWs. Para asegurar que este pico no está relacionado con un calentamiento inhomogéneo, se ha iluminado el NW de la figura 4.9-A con distintas potencias del láser de Nd:YAG, pudiéndose observar los espectros Raman obtenidos en la figura 4.12. Para potencias suficientemente bajas como para no inducir un calentamiento apreciable en el $\mathrm{NW}$, el pico adyacente aparece a la misma frecuencia $\left(\sim 495 \mathrm{~cm}^{-1}\right)$, y tal y como podemos observar en la figura 4.12-B), la intensidad relativa entre el pico Raman del silicio y el adyacente se mantiene constante con independencia de la potencia cuando no hay un efecto de temperatura apreciable. Por otro lado, cuando la potencia induce una temperatura apreciable en el pico Raman del silicio (ver Fig 4.12-A), $500 \mu \mathrm{W})$, i.e. aparece un desplazamiento a bajas frecuencias del pico Raman perteneciente al silicio, el pico adyacente también se desplaza en la misma medida.

Dado que en el NW de la figura 4.7-A) no hay rastro de este pico suplementario, uno podría pensar que su aparición es algo exclusivo de este NW depositado en el substrato de aluminio. Sin embargo, la aparición de picos suplementarios en el espectro Raman de NWs de silicio es un efecto que ha sido reportado por otros autores, aunque no tiene una explicación clara [9, 34-37]. Para investigar si el pico adyacente aparece en otros NWs de nuestras muestras, y si depende de algún modo del substrato, se depositaron sobre un substrato de germanio puro NWs crecidos con oro-galio como catalizador con el fin de analizar su espectro Raman. La elección del germanio como substrato se basa en que conserva las buenas propiedades térmicas que presenta el substrato de aluminio, por lo que si el contacto entre el NW y el substrato es bueno, la disipación de energía debe ser eficiente. En la figura 4.13 se muestran los espectros obtenidos en dos NWs de silicio diferentes a los 


\section{ESPECTROSCOPIA RAMAN EN NWS DE SILICIO Y SILICIO-GERMANIO}
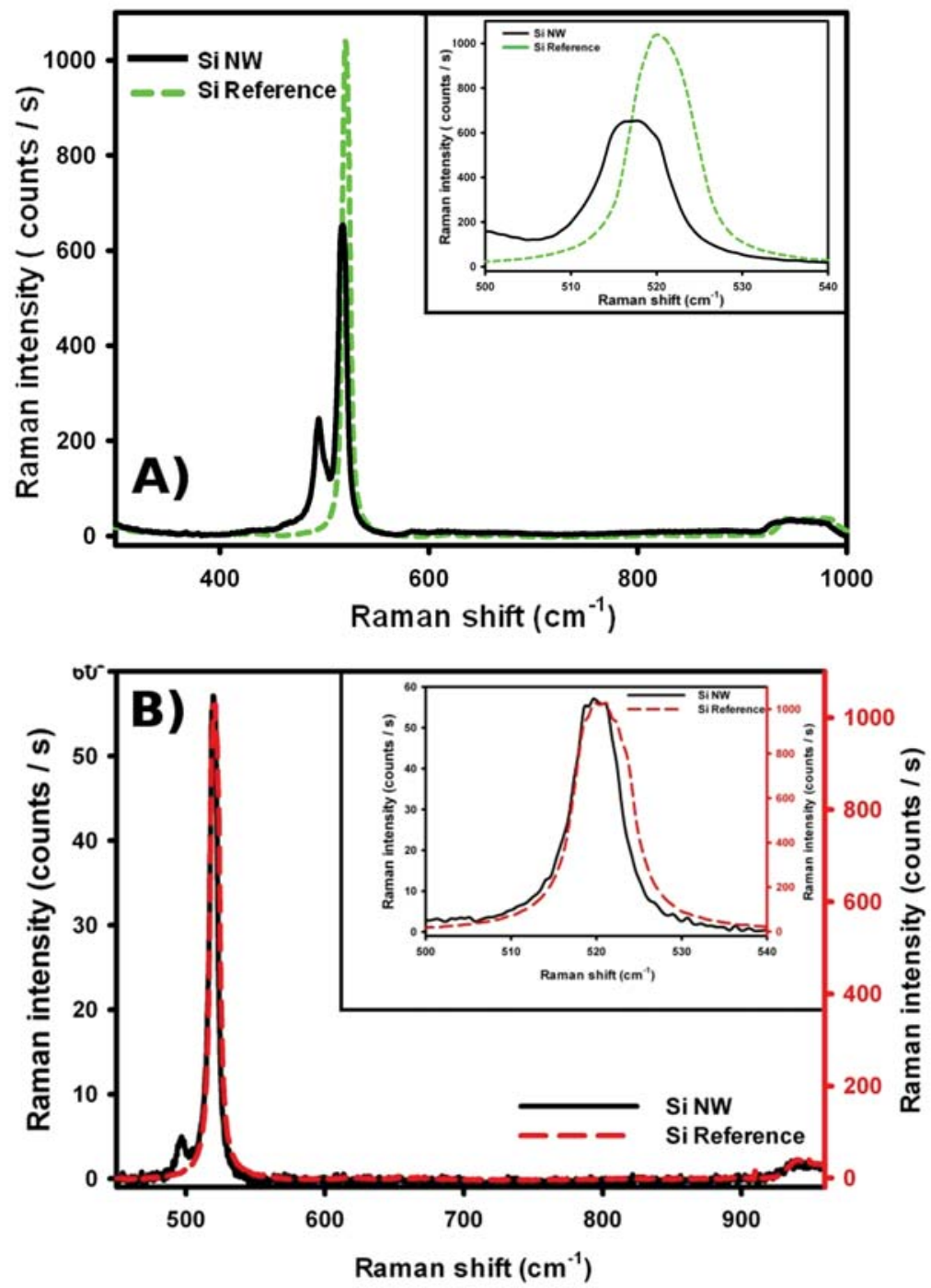

Figura 4.11: Espectros Raman procedentes del NW mostrado en la figura 4.9-A). En A) se muestra el espectro adquirido con el láser de Ne:YAG y una potencia de $500 \mu \mathrm{W}$, mostrando como aparece un pico secundario entorno a $495 \mathrm{~cm}^{-1}$ que no tiene correspondencia en el espectro Raman del silicio volúmico, mostrado en en verde en la misma figura. Por otro lado, las intensidades obtenidas para los espectros del NW y del silicio de referencia están representadas en la misma escala, mostrando la enorme intensidad Raman procedente del NW. B) Espectro Raman en el mismo punto del NW en el que se ha obtenido A) pero utilizando en este caso el láser de $\mathrm{He}-\mathrm{Ne}$ y $1300 \mu \mathrm{W}$ de potencia. Puede observarse como aparece el mismo pico adyacente, pero con una intensidad mucho menor que en A). Se muestra también la comparación de intensidades para la misma potencia entre el NW (escala izquierda) y el silicio referencia (escala derecha), mostrando la debilidad de la señal procedente del NW en este caso. 

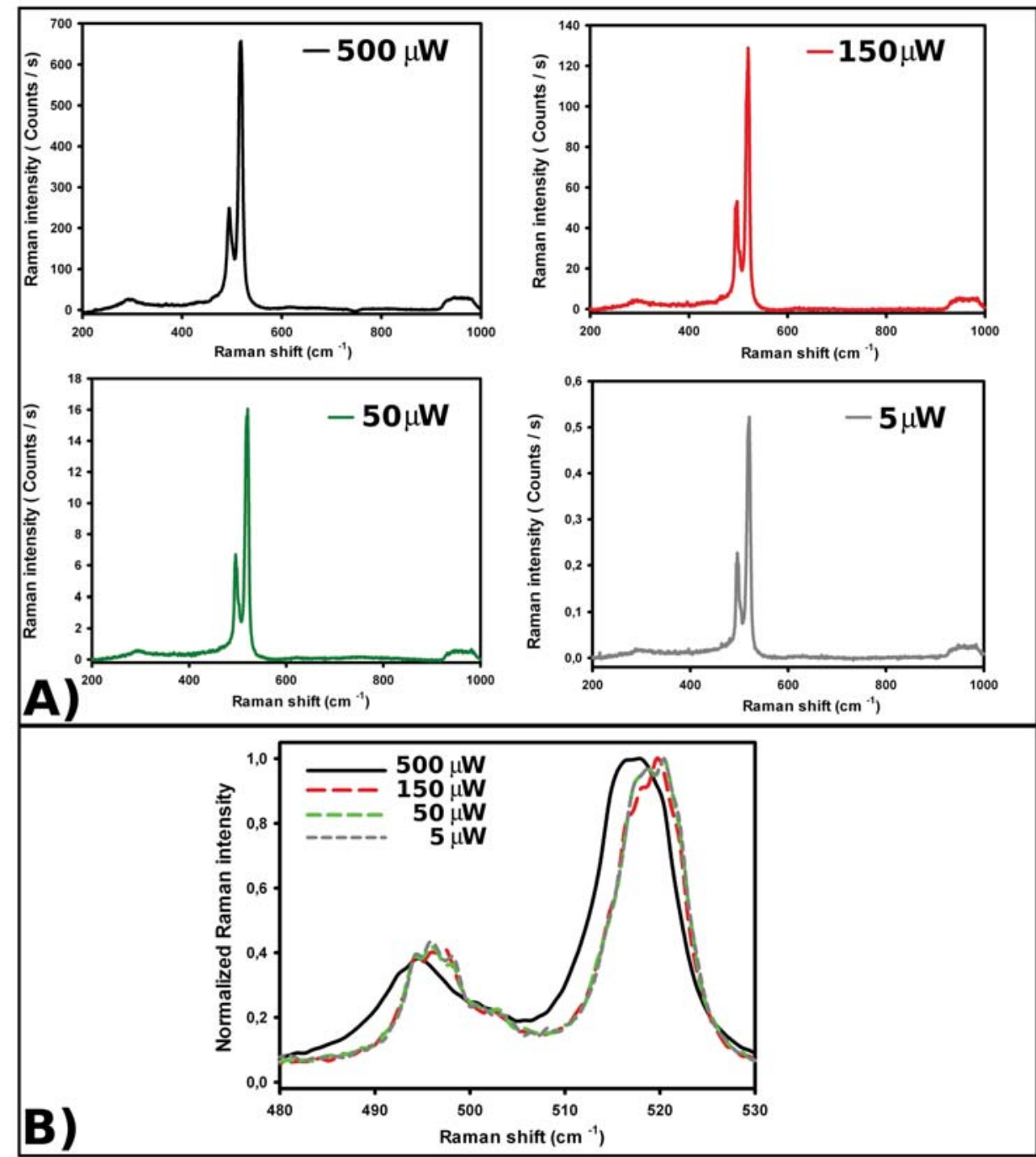

Figura 4.12: A) Espectros Raman en función de la potencia del haz láser. Puede observarse como la relación entre las posiciones del pico Raman del silicio y del pico adyacente se mantiene invariable con independencia de la potencia del haz láser. B) Espectros Raman en un rango espectral reducido para observar mejor como para bajas potencias el espectro Raman obtenido es independiente de la potencia del haz láser incidente. 


\section{ESPECTROSCOPIA RAMAN EN NWS DE SILICIO Y SILICIO-GERMANIO}

mostrados hasta el momento, uno de $52 \mathrm{~nm}$ y algo menos de $10 \mu \mathrm{m}$ de longitud que se encuentra sobre un substrato de aluminio y otro de $125 \mathrm{~nm}$ de diámetro y entorno a $5 \mu$ de longitud depositado en un substrato de germanio puro. Ambos se encuentran aislados y han sido iluminados con el láser de Nd:YAG con una potencia de $500 \mu \mathrm{W}$, estando situados en el centro del spot láser. En 4.13-A) podemos observar como para el NW de $52 \mathrm{~nm}$ depositado sobre aluminio aparece de nuevo el pico adyacente a la misma distancia del pico Raman del silicio. Este NW presenta un ligero calentamiento, ya que el pico Raman aparece desplazado y ensanchado con respecto al pico procedente del silicio de referencia, (espectro en verde). En 4.13-B) se demuestra la independencia del pico adyacente con el substrato, dado que en el NW de $125 \mathrm{~nm}$ depositado sobre germanio también aparece claramente el pico adyacente. El contacto térmico en este caso tampoco es suficientemente eficiente como para eliminar el efecto de la temperatura en el espectro Raman del NW, apareciendo ambos picos desplazados varios $\mathrm{cm}^{-1}$ y ensanchados por efecto de la temperatura. Debido a este ensanche simétrico de los picos, el hueco entre ambos es más pequeño, pero la distancia entre máximos se mantiene invariable. Con esto se demuestra que la procedencia del pico adyacente a $495 \mathrm{~cm}^{-1}$ no se ve afectada por el substrato, y si bien la intensidad relativa de este pico frene al del silicio cambia de un NW a otro, la aparición del pico adyacente es común a los distintos NWs procedentes de nuestros colaboradores de la Universidad Politécnica de Madrid. Sin embargo, el NW dela figura 4.7-A que no presenta este pico adyacente proviene de nuestros colaboradores del "InstitutedePhysique" de la Universidad de Rennes. Por tanto, la aparición de este pico debe estar relacionada con como están fabricados los NWs, i.e. de su estructura cristalina o de sus características superficiales o geométricas. Precisamente, la aparición de este pico secundario ha sido atribuida a la existencia de defectos en la estructura cristalina de los NWs por diversos autores. Lopez et al. mostraron en [34] como en NWs de silicio crecidos por el método VLS, y con una gran densidad de stacking faults aparecen picos suplementarios; en concreto, cuando las stacking faults forman dominios periódicos y aparecen politipos $2 \mathrm{H}$ (wurtzita) de silicio en el NW, se observa claramente la aparición de un pico adyacente en el espectro Raman entorno a $496 \mathrm{~cm}^{-1}$. Cuanto mayor es la fase $2 \mathrm{H}$, más próximo a $495 \mathrm{~cm}^{-1}$ y más intenso aparece el pico Raman. La atribución del pico adyacente a la fase wurtzita en los NWs estudiados en 


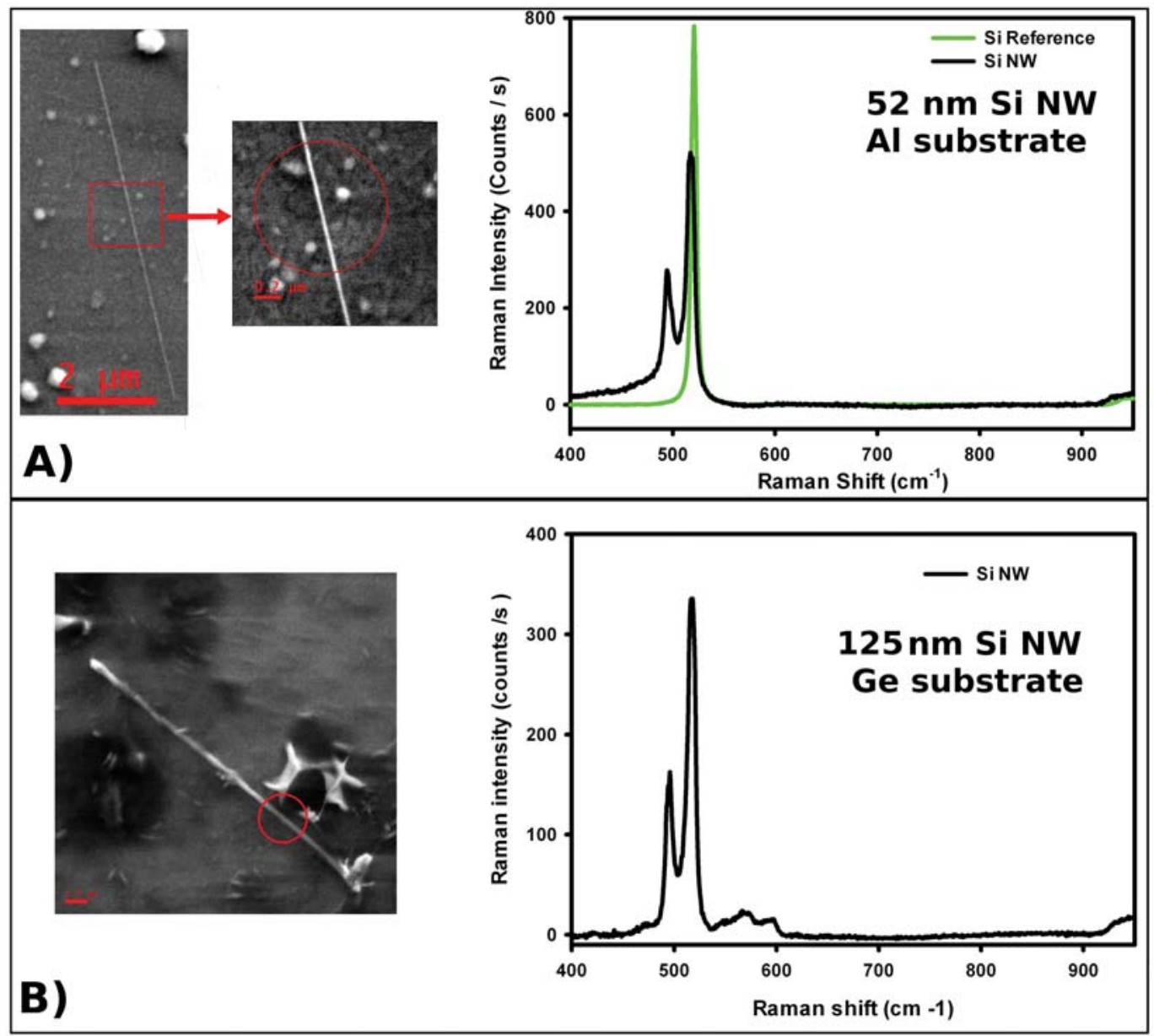

Figura 4.13: A) Espectro Raman de un NW de silicio de $52 \mathrm{~nm}$ y $10 \mu \mathrm{m}$ de longitud depositado en un substrato de aluminio. En verde se muestra el espectro procedente del silicio de referencia en la misma escala de intensidades. La imagen SEM corresponden al NW analizado. B) Espectro Raman de un NW de $125 \mathrm{~nm}$ y $5 \mu \mathrm{m}$ de longitud depositado sobre un substrato de germanio. A la izquierda su correspondiente imagen SEM 


\section{ESPECTROSCOPIA RAMAN EN NWS DE SILICIO Y SILICIO-GERMANIO}

[34] parece muy evidente, dado que para un mismo NW que presenta una mitad sin stacking faults y otra con una gran cantidad de wurtzita, el pico Raman adyacente solo aparece en el espectro tomado en la parte rica en wurtzita [34]. Por otro lado, Prades et al. ya habían mostrado con anterioridad que la existencia de distintas fases hexagonales de Silicio en los NWs pueden causar la aparición de picos Raman adyacentes en el espectro Raman procedente de los NWs de silicio[36]; sin embargo su pico adyacente se situaba a $506 \mathrm{~cm}^{-1}$. Anteriormente a estos resultados, Li et al. habían advertido la aparición de un pico adyacente en los NWs de silicio también a $495 \mathrm{~cm}^{-1}$ [9], el cual atribuyeron a la existencia de silicio amorfo en la superficie de los NWs.

En nuestro caso, la atribución del pico adyacente que observamos en nuestras medidas experimentales a una contribución dada por el silicio amorfo está fuera de lugar, ya que el pico del silicio amorfo es una banda muy ancha [38], que no corresponde con los picos bien definidos que se observan en las figuras 4.12 y 4.13. Por tanto, atribuir este pico a la presencia de politipos debidos stacking faults o twins parece una explicación más plausible. Para probar si este pico adyacente está relacionado con defectos (stacking faults, twins...) en los NWs, se han analizado dos tipos, diferentes en cuanto a su cristalinidad, de NWs de silicio mediante espectroscopia Raman. Nuestros colaboradores de la Universidad Politécnica de Madrid utilizan dos métodos para crecer los NWs, uno basado en utilizar nanoparticulas de oro como catalizador y otro basado en nanoparticulas de una aleación de oro-galio como catalizador. En a figura 4.14 podemos ver la diferencia a nivel de cristalinidad existente entre los NWs crecidos con ambos métodos. En los NWs crecidos con oro como catalizador (Figura 4.14-A)), la bola de oro no siempre se mantiene alineada durante el crecimiento del NW, lo que puede generar staking faults ó twins equivalentes a las vistas en los NWs en los que aparece el pico secundario en la bibliografía [34,39]. Además el análisis del TEM de estos NWs revela que a lo largo de todo el volumen existe silicio en fase wurtzita. Por tanto, atendiendo a la explicación dada en $[34,36]$, en estos NWs uno puede esperar que aparezca el pico adyacente de forma muy intensa. Mientras tanto, los NWs crecidos utilizando nanoparticulas de oro-galio tienen unas propiedades cristalinas mucho mejores (ver figura 4.14-B)). En ellos la bola de oro-galio se mantiene perfectamente alineada 


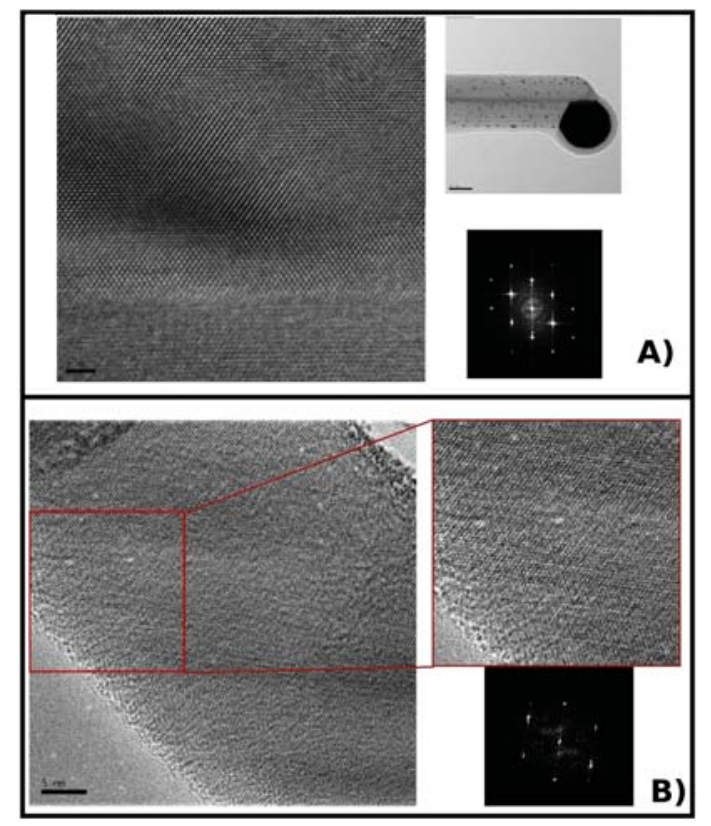

Figura 4.14: Imágenes TEM de NWs de silicio crecidos mediante el método VLS sobre substratos de silicio (111). A) NWs crecidos utilizando oro como semilla, en ellos se observan regiones amorfas y densamente pobladas de defectos, especialmente en la zona central. B) NWs crecidos utilizando $A u_{0,5} G a_{0,5}$ como semilla. En este tipo de NWs se observa una estructura monocristalina y libre de defectos. 


\section{ESPECTROSCOPIA RAMAN EN NWS DE SILICIO Y SILICIO-GERMANIO}

durante todo el proceso de crecimiento, por lo que no introduce defectos en el crecimiento [39]; además, el análisis del TEM realizado en estos NWs revela que la cristalinidad es muy buena, sin defectos ni presencia relevante de wurtzita u otros politipos. Por tanto, en estos NWs no debería de aparecer el pico adyacente, o de hacerlo debería de ser mucho menos intenso que en el espectro adquirido en NWs crecidos únicamente con oro como catalizador. El resultado de este experimento puede verse en la figura 4.15. En 4.15-A) se muestra el espectro obtenido en un NW de silicio crecido utilizando oro como catalizador, el láser utilizado es el Nd:YAG con una potencia de $146 \mu \mathrm{W}$ para no perturbar el espectro con el calentamiento del NW. Puede observarse como en este NW aparece el pico adyacente a $495 \mathrm{~cm}^{-1}$, lo que podría confirmar su relación con los defectos del NW. Sin embargo, la figura 4.15-B), muestra que cuando se excita con el mismo láser un NW de silicio crecido con oro-galio aparece el pico adyacente en la misma posición $\left(495 \mathrm{~cm}^{-1}\right)$, pero con una intensidad mucho mayor, lo que contradice está hipótesis. Por tanto, dado que estos NWs crecidos con oro-galio no incorporan los defectos a los que se atribuye la aparición del pico adyacente, debemos suponer que la aparición de esté pico tiene otro origen. Además, la atribución del pico 495 aquí observado a la existencia de fases wurtzita en el interior del NW tiene otro problema; la frecuencia del pico Raman correspondiente a la wurtzita es mucho más sensible a la potencia/temperatura que el pico correspondiente al silicio cúbico como se demuestra en [40], sin embargo en la figura 4.12 y en 4.13-B) podemos observar como ambos picos tienen una dependencia muy similar con la temperatura, manteniéndose la separación espectral entre los picos adyacente y Si prácticamente invariable.

Otra posible causa que podrían originar la aparición de este pico adyacente junto al pico Raman del silicio sería la existencia de una componente muy tensionada en la estructura, sin embargo nada justifica que en los NWs existan tensiones tan grandes como para tener un desplazamiento del pico tensionado hasta $495 \mathrm{~cm}^{-1}$ [41]. Por ultimo, el estudio realizado por Peng et al. en [37] constituye el análisis más detallado del origen de este pico adyacente a $495 \mathrm{~cm}^{-1}$ del que se tiene noticia. Dado que el pico no puede pertenecer a una contribución de silicio amorfa del interior del NW, Peng et al. estudiaron la posibilidad de que el pico fuese un efecto de superficie, centrándose en la posibilidad de que el silicio en superficie formase enlaces con hidrógeno, que dan un pico Raman entorno a $495 \mathrm{~cm}^{-1}[42,43]$. 

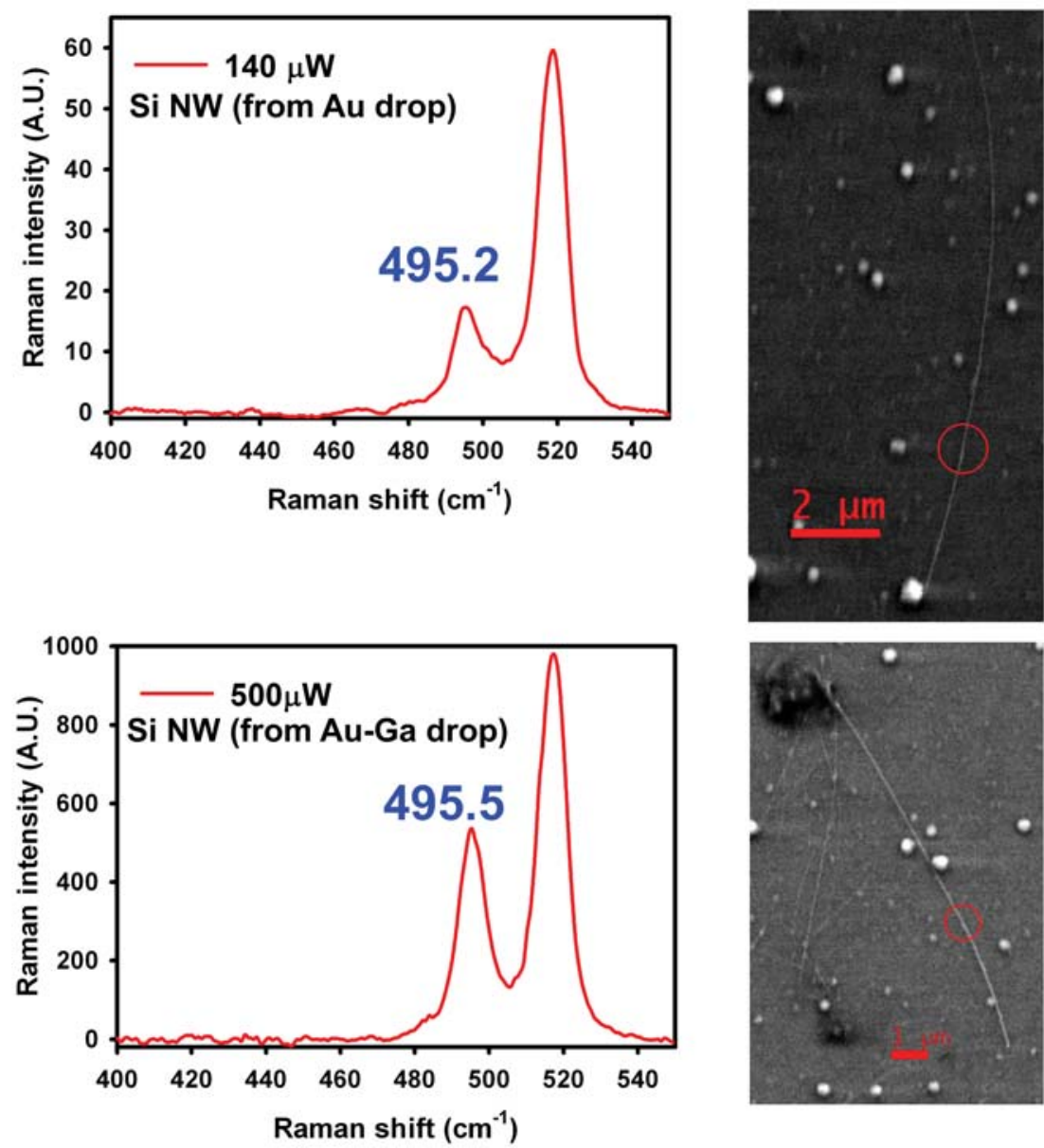

Figura 4.15: A) Espectro Raman adquirido de un NW de silicio crecido con oro como catalizador y depositado sobre aluminio. B) Espectro Raman adquirido sobre un NW de silicio crecido con oro-galio como catalizador. 


\section{ESPECTROSCOPIA RAMAN EN NWS DE SILICIO Y SILICIO-GERMANIO}

Para eliminar este efecto, sometieron a los NWs a diferentes tratamientos superficiales destinados a romper cualquier enlace de este tipo. Sin embargo, y pese a los distintos ataques, no consiguieron hacer desparecer del espectro Raman el pico adyacente, por lo que finalmente concluyeron que la aparición del pico adyacente se debe a una combinación de la existencia de pequeños nanocristales de silicio y a zonas policristalinas próximas a la superficie [37]. Sin embargo, la contribución de los nanocristales presentaría una asimétrica por confinamiento fononico en el pico adyacente que no se observa en nuestros espectros [21]; además, si el pico estuviera relacionado con politipos locales, cuanto más intensamente aparezca el pico adyacente más desplazado a bajas energías y más ancho debería aparecer el pico principal, sin embargo en todos los espectros mostrados se puede apreciar la independencia de ambos picos en esos términos, por lo que esta explicación también tiene sus puntos débiles a la hora de explicar el origen del pico adyacente observado en estos NWs. Un efecto que puede tener una gran relevancia a la hora de explicar el origen de este pico mostrado en [37], es que el pico adyacente solo aparece cuando someten el NW a un ataque por medio de ácido hidrofluorídrico (HF) [37], no apareciendo en NWs de silicio cuya superficie no es atacada. Como ya se ha visto en capítulo 2, la superficie juega una papel muy relevante en el comportamiento de los fonones en los NWs semiconductores, siendo especialmente relevante el papel jugado por la rugosidad de la superficie. Cuando se realiza un ataque HF en la superficie de los NWs, se está modificando la morfología de la superficie, y por tanto la rugosidad del NW. Por tanto, puede que ese sea el origen de la aparición del pico 495 en [37], y la justificación de por que solo aparece en determinados NWs de silicio. Ahondando en esta hipótesis, se sabe que en NWs semiconductores del grupo III-V se han reportado numerosos casos en los que en espectro Raman muestra una banda adyacente, estando relacionada su aparición con la existencia de fonones de superficie/polaritones $[4,44,45,46]$. Estos se producen por un acoplamiento electromagnético entre los fonones y los fotones de haz láser, y son muy dependientes del campo electromagnético local [46]; además, la rugosidad de la superficie puede aumentar la intensidad Raman con la que observamos estos picos Raman debidos a efectos de superficie $[4,47]$. En el silicio, aunque más débil en principio, también tiene un fonón de superficie característico que aparece en el espectro Raman precisamente a $495 \mathrm{~cm}^{-1}$ [48]; por tanto, teniendo en cuenta la enorme influencia 
de la morfología superficial de los NWs de silicio en el comportamiento fonónico, no podemos descartar que la aparición del pico adyacente observado en algunos NWs de silicio este relacionada con una contribución debida a este fonón de superficie, cuya intensidad Raman está siendo amplificada bebido a una resonancia del campo electromagnético localizada en la superficie del NW [46]; precisamente, la existencia de este efecto de resonancia del campo eléctrico en superficie para determinados diámetros del NW será demostrada en el capítulo 5.

La interpretación de este pico adyacente como una contribución de un fonón de superficie implica que la intensidad del pico tiene que depender fuertemente de las condiciones que afectan al campo electromagnético en la superficie del NW, como pueden ser el diámetro del NW, o las características del campo electromagnético incidente. Dentro de estas últimas, cabe esperar una gran dependencia de la intensidad de este pico con la frecuencia de los fotones incidentes. En la figura 4.11, se mostró entre otras cosas que la intensidad relativa del pico adyacente frente al pico Raman del silicio dependía de la longitud de onda del láser que ilumina al NW. En la figura 4.16 podemos ver que este comportamiento se confirma para otros NWs de silicio crecidos utilizando oro-galio como catalizador. La caracterización Raman se ha realizado empleado los láseres de Nd:YAG y de He-Ne, con unas potencias de $500 \mu \mathrm{W}$ y $1300 \mu \mathrm{W}$ respectivamente. Puede observarse en la figura 4.16-A) y 4.16-B) como para este NW también aparece el pico adyacente a $\sim 495 \mathrm{~cm}^{-1}$ con independencia de la longitud de onda utilizada. Sin embargo, la relación de intensidades entre el pico Raman del silicio y el adyacente no se conserva al cambiar la longitud de onda, al igual que se observaba en el NW de la figura 4.11; por tanto, la intensidad de este pico es dependiente de la longitud de onda con la que se ilumina el NW, demostrando por tanto que el efecto que lo causa tiene que depender de la longitud de onda del haz láser incidente.

\subsubsection{Intensidad Raman procedente de los NWs comparada con la intensi- dad esperada.}

Otro punto importante concerniente al espectro Raman procedente de los NWs de silicio, y que resulta anómalo frente al espectro obtenido en el silicio de referencia, es la gran intensidad con la que se observa el pico Raman en los NWs. La 


\section{ESPECTROSCOPIA RAMAN EN NWS DE SILICIO Y SILICIO-GERMANIO}
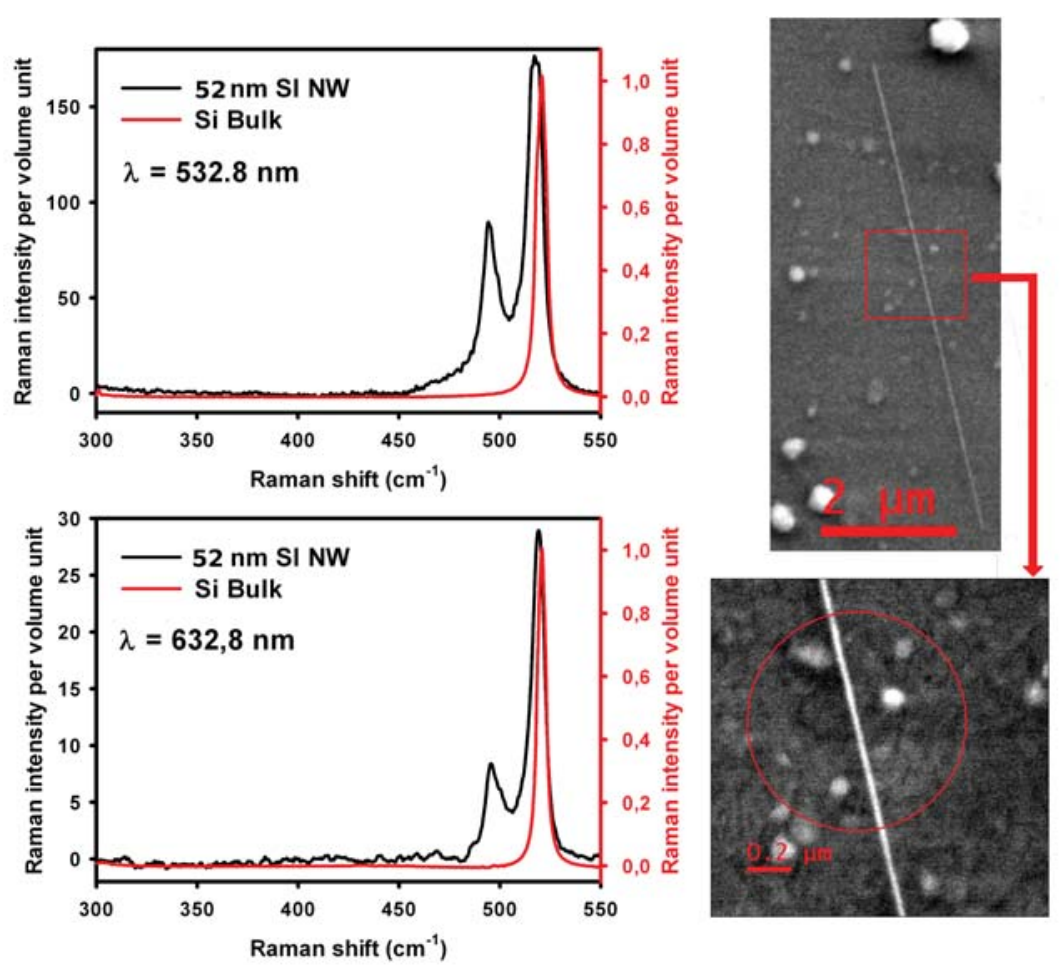

Figura 4.16: Espectros Raman adquiridos sobre un NW de silicio crecido con orogalio y depositado en un substrato de aluminio. Los espectros han sido adquiridos utilizando dos longitudes de onda para el haz láser, $532.8 \mathrm{~nm}$ (Nd:YAG) y $632 \mathrm{~nm}$ (He-Ne) con unas potencias de $500 \mu \mathrm{W}$ y $1300 \mu \mathrm{W}$ respectivamente. La escala de intensidad Raman se muestra en intensidad Raman por unidad de volumen iluminado. La escala perteneciente a los NWs se encuentra a la izquierda, (negro), mientras que los espectros tomados del silicio de referencia se muestran en la escala de la derecha (roja). 
señal Raman obtenida en el NW mostrado en la figura 4.16 muestra una intensidad comparable a la obtenida en el silicio de referencia cuando se utiliza el láser de Nd:YAG. Este mismo comportamiento se observaba también en los distintos NWs de las figuras 4.11-A), 4.13 y 4.15; resultando en un comportamiento muy llamativo, dado que el volumen de material excitado en los NW es mucho menor que el volumen de material excitado en una muestra volúmica. Dado que el scattering Raman es una interacción de segundo orden, y por tanto de relativamente escasa probabilidad de ocurrencia [49], el disponer de un mayor volumen de material susceptible de ser excitado implica obtener una mayor cantidad de fotones Raman, y por tanto una mayor intensidad de la señal recogida. Dado que el volumen iluminado en los NWs es muy pequeño en comparación con el volumen de material iluminado para obtener el espectro de referencia, la señal Raman procedente de los NWs debería ser mucho más débil en comparación. Para poder ver mejor este efecto, vamos a calcular la intensidad Raman por unidad de volumen en los NWs , para posteriormente, compararla con la intensidad Raman por unidad de volumen obtenida en la muestra de referencia. El volumen excitado en una muestra macroscópica está determinado en el plano por el área de muestra iluminada por el láser (diámetro del spot), mientras que en profundidad lo está por el coeficiente de absorción. Además, uno debe tener en cuenta que los fotones procedentes del scattering Raman en la muestra también son absorbidos al atravesar el material, de tal modo que de acuerdo con la expresión dada por De Wolf et al. [23] la profundidad efectiva del volumen que contribuye a la señal Raman puede calcularse como:

$$
d_{p}=\frac{-\log (0,1)}{2 \alpha}
$$

Utilizando esta profundidad puede hacerse la relación entre los volúmenes excitados en el NW y en la muestra de referencia para cada longitud de onda utilizada, y con ello podemos encontrar la intensidad Raman por unidad de volumen excitado. En la figura 4.16, la escala en la que se muestran las intensidades Raman se encuentra expresada utilizando la intensidad Raman por unidad de volumen, pudiéndose observar que este NW el pico Raman tiene una intensidad muy superior a lo que le correspondería por el volumen de material que está siendo excitado. Este efecto es mayor para el láser de Nd:YAG, el cual presenta una intensidad Raman 150 veces 


\section{ESPECTROSCOPIA RAMAN EN NWS DE SILICIO Y SILICIO-GERMANIO}

mayor que lo esperado por su volumen, mostrando que el efecto es dependiente de la longitud de onda que ilumina al NW. Gracias a este efecto, se explica que haya sido posible realizar una caracterización Raman utilizando unas potencias tan bajas como $5 \mu \mathrm{W}$ en un tiempo de adquisición razonable. Además, cuando se compara la señal obtenida bajo las mismas condiciones de iluminación para un NW de 52 nm de diámetro (Figura 4.13-A) ) y para otro de $125 \mathrm{~nm}$ (Figura 4.13-B)) se puede observar como ambos tienen una intensidad similar pese a que el volumen iluminado en el segundo es casi 6 veces superior al del primero. Por tanto, la intensidad Raman de los NWs parece depender fuertemente del diámetro del NW. Resultados similares para NWs de silicio y nanoconos de silicio han sido reportados por Cao et al. [50,51], donde experimentalmente muestran la existencia de una dependencia de la intensidad Raman por unidad de volumen con el diámetro de los NWs de silicio, y con la longitud de onda del haz utilizado. En este trabajo, muestran como la intensidad por unidad de volumen aumenta rápidamente en los NWs al disminuir el diámetro, apreciándose valores próximos compatibles con los calculados en nuestros NWs. Por otro lado, la intensidad por unidad de volumen de los NWs aumenta cuando la longitud de onda del láser incidente es mayor [50,51]; sin embargo, en la figura 4.16 puede observarse como en nuestro caso esto no sucede así, siendo mucho más intensa la señal obtenida con el láser Nd:YAG de $532 \mathrm{~nm}$ que con el He-Ne de $632.8 \mathrm{~nm}$. El efecto de la gran intensidad Raman observada en NWs de silicio es descrito por Cao et al. en términos de la interacción electromagnética del NW (dieléctrico) con el haz láser (campo electromagnético), asumiendo que este puede describirse a través del formalismo del scattering de Mie para nanopartículas esféricas $[50,51]$. El cálculo del campo electromagnético mediante este formalismo en el interior del NW se realiza asumiendo que este es infinito, y conlleva a la aparición de una serie de resonancias dependientes del diámetro que elevan el campo eléctrico en el interior del NW, justificando así la aparición de una señal tan intensa en los NWs. Sin embargo, la solución teórica resulta en una curva para la intensidad por unidad de volumen de los NWs con fuertes oscilaciones en función del diámetro y de la longitud de onda. Este efecto impide establecer que la intensidad relativa sea siempre mayor para la longitud de onda 632.8 que para la 532, ya que el ratio entre ellas va a ser muy dependiente del radio del NW [50,51]. Por otro lado, y dado que el aumento de la señal Raman parece estar relacionado con 
un efecto electromagnético, uno podría pensar que el substrato de aluminio puede contribuir a que la señal sea tan grande, ya que al ser un metal podría aparecer algún efecto de tipo Raman-resonante en la interacción láser-NW-substrato. Además, el aluminio ha sido utilizado como substrato por sus características superficiales para obtener efectos resonantes (SERS) en la señal Raman de muestras depositadas sobre él [52]. Para eliminar/cuantificar este posible efecto introducido por el substrato, se ha realizado un experimento consistente en cambiar el substrato sobre el que se depositan los NWs por otro que no es susceptible de presentar estos acoplamientos electromagnéticos; eligiéndose para tal fin un semiconductor no dopado como es el germanio puro. Podemos ver el resultado de este experimento en la figura 4.17, donde un NW de silicio de $110 \mathrm{~nm}$ de diámetro se encuentra depositado sobre un substrato de germanio puro. En el espectro aparece simultáneamente el pico Raman del germanio a $\sim 300 \mathrm{~cm}^{-1}$ perteneciente al substrato, y el pico Raman procedente del NW de silicio a $\sim 520 \mathrm{~cm}^{-1}$ junto al pico suplementario. Incluso teniendo en cuenta que para el láser de Nd:YAG el germanio tiene una señal Raman mucho mas intensa que el silicio, las intensidades del germanio de referencia y del NW de silicio se encuentra en un orden de magnitud comparable, resultando en una intensidad Raman por unidad de volumen $\sim 168$ veces la del silicio de referencia, muy próximo a lo observado para el NW de silicio de $52 \mathrm{~nm}$ sobre un substrato de aluminio. Por tanto, la enorme intensidad obtenida de los NWs de silicio no parece estar relacionada con un efecto SERS introducido por el substrato de aluminio bajo el que se encuentra el NW. Sin embargo, en el capítulo 5 podremos ver como el substrato, a través del acoplamiento electromagnético en la interacción láser-NW-substrato, tiene un papel dominante en la enorme intensidad Raman observada.

\subsection{Espectroscopia Raman en NWs de $S i_{1-x} G e_{x}$ •}

El espectro Raman procedente de las aleaciones de silicio-germanio es más complejo de analizar que el obtenido de sus componentes por separado. El espectro Raman de la aleación presenta un carácter multimodo [53 - 55], caracterizado por la aparición de tres lineas principales situadas entorno a $\sim 290 \mathrm{~cm}^{-1}, \sim 407 \mathrm{~cm}^{-1} \mathrm{y}$ $481 \mathrm{~cm}^{-1}$ cuando la aleación contiene un $50 \%$ de cada elemento, atribuidas a las vibraciones de los enlaces Ge-Ge, Si-Ge y Si-Si [55,56]. Además de estos picos 


\section{ESPECTROSCOPIA RAMAN EN NWS DE SILICIO Y SILICIO-GERMANIO}
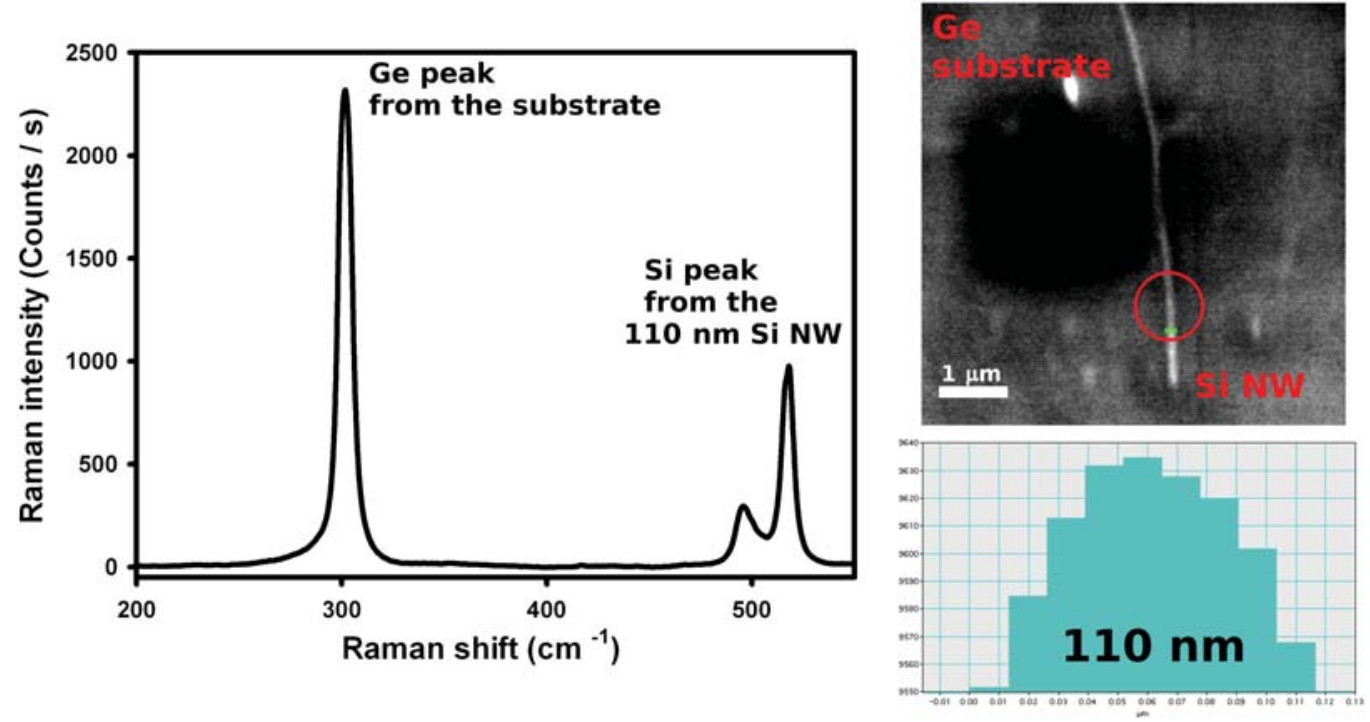

Figura 4.17: Espectro Raman tomado en un NW de silicio de $110 \mathrm{~nm}$ de diámetro depositado en un substrato de germanio puro. El láser utilizado ha sido el Nd:YAG con una potencia de $500 \mu \mathrm{W}$, encontrándose el NW centrado en el spot láser. Nótese la intensidad Raman del NW en comparación con el pico Raman procedente del substrato de germanio 
principales, pueden aparecer otros con menor intensidad entre los picos del $\mathrm{Ge}-\mathrm{Ge}$ y Si-Si, de cuya naturaleza no se se tiene un conocimiento claro; algunos autores los atribuyen al desorden de la aleación [55], mientras que otros autores los atribuyen al efecto contrario, atribuyéndolos a la existencia de ciertas estructuras ordenadas correspondientes a zonas con una alta densidad de enlaces Si-Si rodeadas de átomos de germanio [56]. Por otra lado, las intensidades relativas y las frecuencias

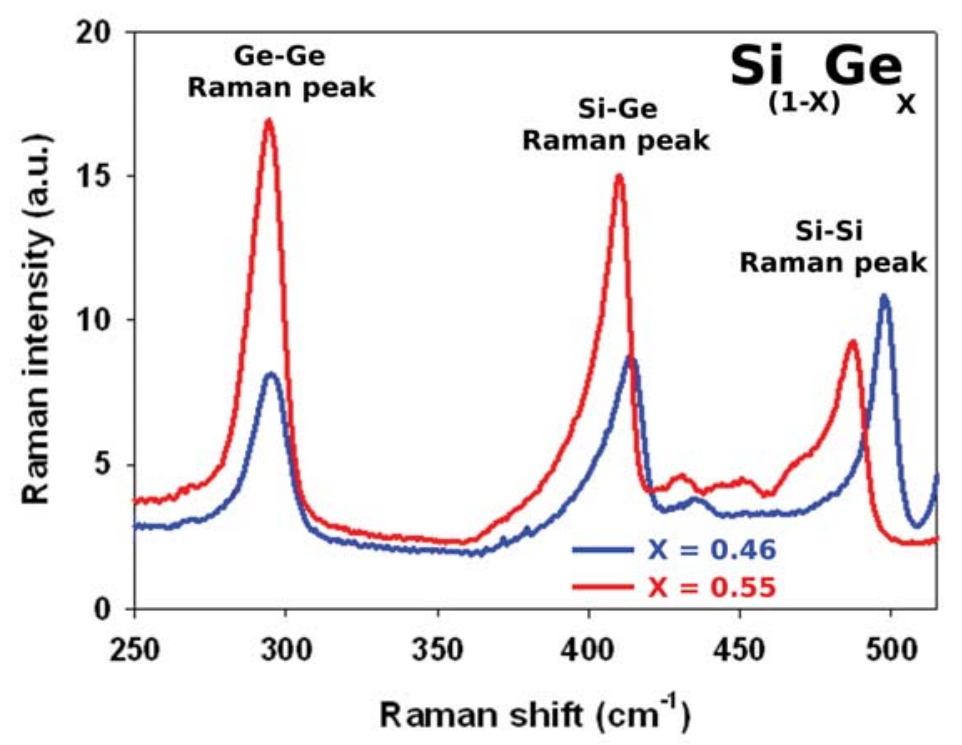

Figura 4.18: Espectro Raman adquirido sobre dos muestras de silicio-germanio con dos composiciones diferentes, $S i_{56} G e_{44}$ y $S i_{45} G e_{55}$. Puede apreciarse la aparición de tres picos diferenciados, correspondientes a los enlaces $\mathrm{Si}-\mathrm{Si}$, Si-Ge y Ge-Ge, y la existencia de otros picos mucho más débiles entre los picos $\mathrm{Si}-\mathrm{Si}$ y Si-Ge. Nótese como al aumentar la cantidad de germanio el pico Si-Si se debilita y se desplaza a bajas frecuencias, mientras que el Ge-Ge aumenta de intensidad y se desplaza a latas frecuencias.

correspondientes a los picos principales son fuertemente dependientes de la composición de aleación; podemos ver este efecto en la figura 4.18, donde se muestran los espectro Raman adquiridos en muestras volúmicas de silicio-germanio con dos composiciones muy cercanas de $S i_{56} G e_{44}$ y $S i_{45} G e_{55}$. El pico correspondiente a los enlaces Si-Si aumenta en intensidad y se desplaza a la frecuencia del silicio puro cuando se disminuye la proporción de germanio en la aleación, al mismo tiempo que el pico Ge-Ge se debilita y se desplaza a bajas frecuencias. Esto se debe al des- 


\section{ESPECTROSCOPIA RAMAN EN NWS DE SILICIO Y SILICIO-GERMANIO}

censo del parámetro de red del cristal cuanto menos germanio tiene la aleación, y por otro lado a la aparición de clusters de silicio puro [57]. Las posiciones de estos picos en función de la composición de la aleación fueron cuidadosamente estudiadas por Brya [54], a partir de cuyos resultados se ha construido la figura 4.19. A
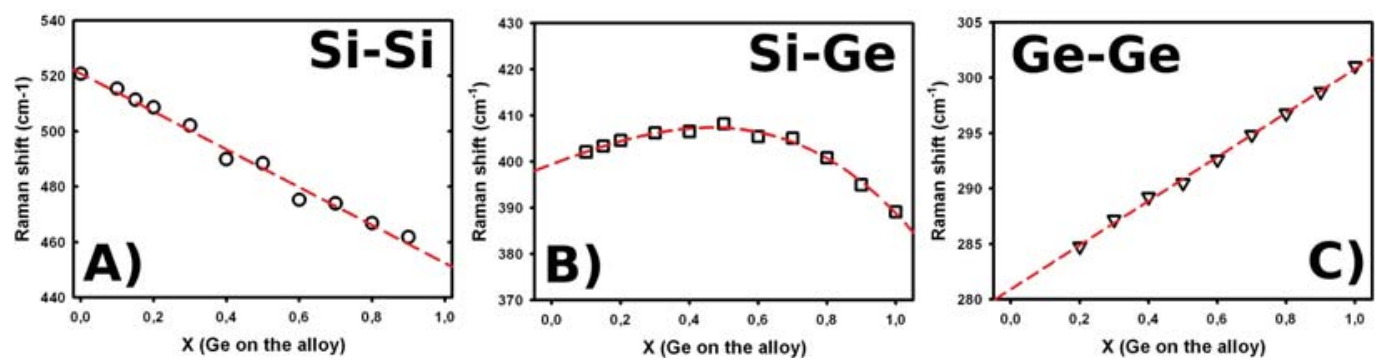

Figura 4.19: Frecuencias a las que aparecen los picos Raman correspondientes a los enlaces $\mathrm{Si}-\mathrm{Si}$ (A), Si-Ge (B) y Ge-Ge (C) en función de la cantidad de germanio en la aleación (símbolos). Las curvas muestran los ajustes por mínimos cuadrados que permiten interpolar en cada caso la frecuencia en función de la composición. Los datos experimentales proceden de la referencia [54]

partir de los datos experimentales que aparecen en la figura 4.19, puede estimarse las frecuencias a las que aparecen los picos principales para cualquier proporción de los elementos que conforman la aleación en ausencia de tensiones:

$$
\begin{array}{r}
\omega_{S i-S i}=520,7-67,1 X \\
\omega_{S i-G e}=399,5+28,2 X-11,0 X^{2}-27,8 X^{3} \\
\omega_{G e-G e}=280,9+19,9 X
\end{array}
$$

Estas expresiones son muy similares a las encontradas por otros autores para los picos Si-Si y Ge-Ge [58 - 62], mientras que la frecuencia del pico Si-Ge encierra más controversia en su determinación, existiendo diferentes valores tanto experimentales como teóricos para las posiciones a las que aparece este pico [59-61]. Por tanto, si no tenemos en cuenta al pico SiGe, a partir de las expresiones para $\omega_{S i-S i}(X)$ y $\omega_{G e-G e}(X)$ dadas en 4.4 , uno puede estimar de una forma muy simple la composición de una muestra de silicio-germanio a partir del espectro Raman, permitiendo una caracterización composicional de las muestras mediante espectroscopia Raman. Un factor importante a la hora de analizar el espectro Raman 
procedente de una aleación de silicio-germanio es el papel jugado por las tensiones en la red. El parámetro de red del germanio es entorno a un $4 \%$ más grande que el del silicio, y para muestras con al menos una dimensión muy reducida, pueden aparecer tensiones que desplazan la posición de los picos Raman en muestras de baja dimensionalidad frente a las posiciones características de la aleación no tensionada [63]. Sin embargo, M.Holtz et al. mostraron como al menos por encima de un espesor de $55 \mathrm{~nm}$, no se observa ningún efecto apreciable en las posiciones de los picos Raman adquiridos sobre capas epitaxiales de $S i_{1-X} G e_{X}$ para cualquier valor de $X$ [64]. Por tanto, uno puede utilizar las expresiones dadas en 4.4 para caracterizar composionalmente NWs de $S i_{1-X} G e_{X}$ cuyos diámetros sean del order de los $50 \mathrm{~nm}$ o más sin temor a introducir un error por efecto de las tensiones en la red.

El crecimiento de NWs semiconductores de una aleación requiere un control muy preciso de las condiciones de crecimiento para obtener una composición determinada. Para determinar la composición de los NWs, típicamente se recurre a técnicas de caracterización como el HRTEM+EDX (High-Resolution-Transmision-ElectronMicroscopy + Energy-Dispersive-X-Ray-Spectroscopy), que resultan muy costosas de realizar y que requieren de una delicada interpretación [61,65 - 67]. Por tanto, la posibilidad de realizar un análisis composicional mediante una técnica relativamente más simple, como es la espectroscopia Raman supone un gran paso adelante en la caracterización de estas estructuras.

\subsubsection{Efecto de la temperatura inducida por el haz láser en NWs de $S i_{1-X} G e_{X}$}

Cuando se intenta caracterizar la composición de los NWs de una aleación como el $S i_{1-X} G e_{X}$ a través de la frecuencia característica de los picos Raman, lo primero que uno debe asegurar es que no existen otros efectos que modifiquen la forma de dicho espectro. Ya hemos visto como en NWs de $S i_{1-X} G e_{X}$ cuyos diámetros sean superiores a $50 \mathrm{~nm}$, los efectos de las tensiones introducidas por la diferencia de parámetros de red pueden despreciarse; sin embargo, existe un efecto que desplaza las frecuencias de los picos Raman de un modo para nada despreciable y que no 


\section{ESPECTROSCOPIA RAMAN EN NWS DE SILICIO Y SILICIO-GERMANIO}

es otro que la temperatura [68]. Tal y como hemos visto tanto teórica, como experimentalmente para NWs de silicio, la temperatura que se induce en los NWs al ser iluminados por un haz láser puede modificar por completo la forma de su espectro. La temperatura inducida en los NWs depende de diversos factores, como son la potencia del haz, las condiciones de disipación, la longitud de onda del láser, las dimensiones de los NWs, la conductividad térmica - que para NWs de $S i_{1-X} G e_{X}$ es mucho más baja que para NWs de Si, ver capítulo 2 sección 2.2.3.5, y sobre todo de la posición que ocupan los NWs en el área de iluminación. Por tanto, para caracterizar correctamente los NWs de silicio-germanio, uno tiene que asegurar que no se está modificando el espectro por efecto de temperatura. En la figura 4.20 se muestra una serie de espectros Raman tomados sobre una muestra compuesta por dos NWs cruzados de $S i_{\sim 85} G e_{\sim 15}$ que se encuentran depositados sobre un substrato de aluminio; por tanto, podría pensarse que las condiciones de disipación son buenas. Sin embargo, podemos observar como dependiendo de la posición que ocupan los NWs en el área iluminada por el haz láser, los espectros obtenidos son completamente diferentes. Al estar uno de los NWs montado sobre otro, no tiene un buen contacto con el substrato, y por tanto la energía absorbida del haz láser no puede disiparse, resultando en un incremento de la temperatura muy elevado. Por otro lado, el NW que se encuentra en contacto con el substrato se calienta mucho menos, resultando en un caso donde la señal procede de dos NWs que se encuentran a muy distinta temperatura, apareciendo un desdoblamiento de la banda $\mathrm{Si}-\mathrm{Si}$ en dos picos bien diferenciados; uno muy ancho y desplazado a baja frecuencia, perteneciente al NW sobrecalentado, y otro más estrecho y situado a una frecuencia mayor perteneciente al NW menos caliente.

A la vista de la figura 4.18, queda patente que realizar la caracterización Raman de NWs de silicio-germanio en muestras compuestas por varios NWs no resulta una buena idea. Además del efecto introducido por las diferentes temperaturas que pueden alcanzar cada NW simultáneamente iluminado en función de su posición en el spot láser, cualquier diferencia en la composición de los NWs va a falsear el espectro final; en función de donde se encuentren los NWs y sin considerar el efecto de temperatura, la intensidad con la que cada uno contribuye al espectro final va a ser diferente por estar iluminados de diferente forma dependiendo de donde se situé el haz láser, obteniendo diferentes espectros de la misma muestra. Una vez 

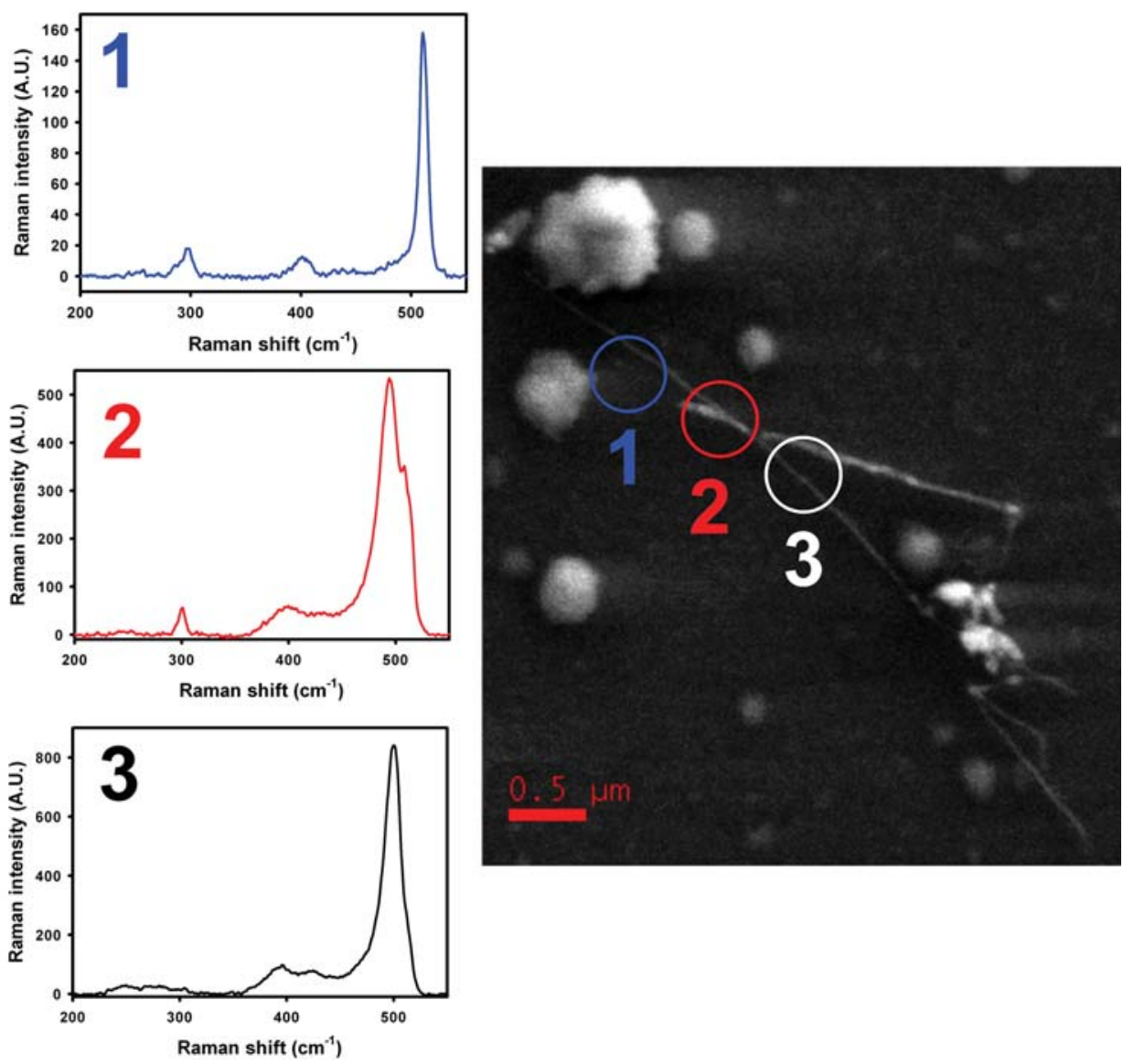

Figura 4.20: Espectros Raman tomados en la muestra compuesta por dos NWs cruzados de $S i_{\sim 85} G e_{\sim 15}$ y mostrada a la derecha (imagen SEM). Puede observarse como dependiendo de la posición que ocupan los NWs en el área iluminada se obtienen unos espectros completamente diferentes. En 1 el láser ilumina de forma exclusiva el NW que se encuentra en contacto con el substrato, mostrando un espectro muy diferente al observado en 2, cuando el láser ilumina a los dos NWs simultáneamente. En este caso se puede apreciar como las diferentes temperaturas alcanzadas por los NWs causan una separación del pico Si-Si en dos. Por último, en 3 el láser ilumina casi exclusivamente al NW que no tiene contacto con el substrato, puede observarse como el pico Si-Si es mucho más grueso que el que aparece en 1 y se encuentra muy desplazado en comparación. El láser utilizado ha sido el Nd:YAG de 532 nm de longitud de onda y con una potencia de $500 \mu \mathrm{W}$. 


\section{ESPECTROSCOPIA RAMAN EN NWS DE SILICIO Y SILICIO-GERMANIO}

establecido que la caracterización de los NWs de silicio-germanio debe realizarse sobre NWs individuales, uno debe probar que las condiciones experimentales no pueden introducir un efecto de temperatura relevante que falsee el espectro propio del NW. Como ya vimos para NWs de silicio, una forma perfectamente válida de asegurar que con una determinada potencia del láser no se induce una temperatura relevante en el NW consiste en barrer transversalmente el NW con el haz láser. Cuando se ilumina el NW con un haz láser gaussiano, la temperatura y la posición del pico Raman en función de la posición que ocupa en el área iluminada siguen el perfil gaussiano del haz (ver figuras 4.8 y 4.10). Por tanto, cuando el perfil del desplazamiento del pico $\mathrm{Si}-\mathrm{Si}$ es independiente de la posición que ocupa el NW en el área de iluminación,e independiente de como se este iluminando el NW, la potencia utilizada no deforma el espectro obtenido por efecto de temperatura, y el espectro Raman es el característico del NW. En la figura 4.21, podemos observar experimentalmente el proceso descrito para determinar una potencia de trabajo que asegure la obtención del espectro Raman del NW eliminando la indeterminación introducida por las condiciones de iluminación. En ella, un NW de $S i_{\sim 88} G e_{\sim 12}$ crecido por el método VLS, con oro-galio como catalizador y $\sim 95 \mathrm{~nm}$ de diámetro, ha sido iluminado por el haz láser de Nd:YAG. La longitud de onda del láser es de $532.8 \mathrm{~nm}$ y se han utilizado dos potencias, $146 \mu \mathrm{W}$ y $500 \mu \mathrm{W}$. El NW se encuentra depositado en un substrato de aluminio, permitiendo una buena disipación de la energía absorbida por del láser. En la figura 4.21-A), podemos ver como cuando el NW se encuentra en el borde del área de iluminación, los espectros mostrados en 1 son idénticos con independencia de la potencia utilizada. Sin embargo, cuando el NW se encuentra en el centro del área de iluminación, el espectro adquirido con la potencia más alta se encuentra desplazado y ensanchado frente al espectro adquirido a una potencia más baja (2). Las frecuencias del pico Raman Si-Si en función de la posición del NW en el área de iluminación se muestra en 4.21-B) para las dos potencias. Puede observarse como cuando se usa la potencia más alta, la frecuencia a la que aparece el pico $\mathrm{Si}-\mathrm{Si}$ depende fuertemente de la posición relativa del NW frente al centro del haz láser; mientras tanto, cuando se ilumina con una potencia de $146 \mu \mathrm{W}$ la frecuencia es independiente de dicha posición relativa. Por lo tanto, realizar un análisis de los cortes transversales es un herramienta fundamental para interpretar correctamente el espectro Raman de los NWs en general, y 

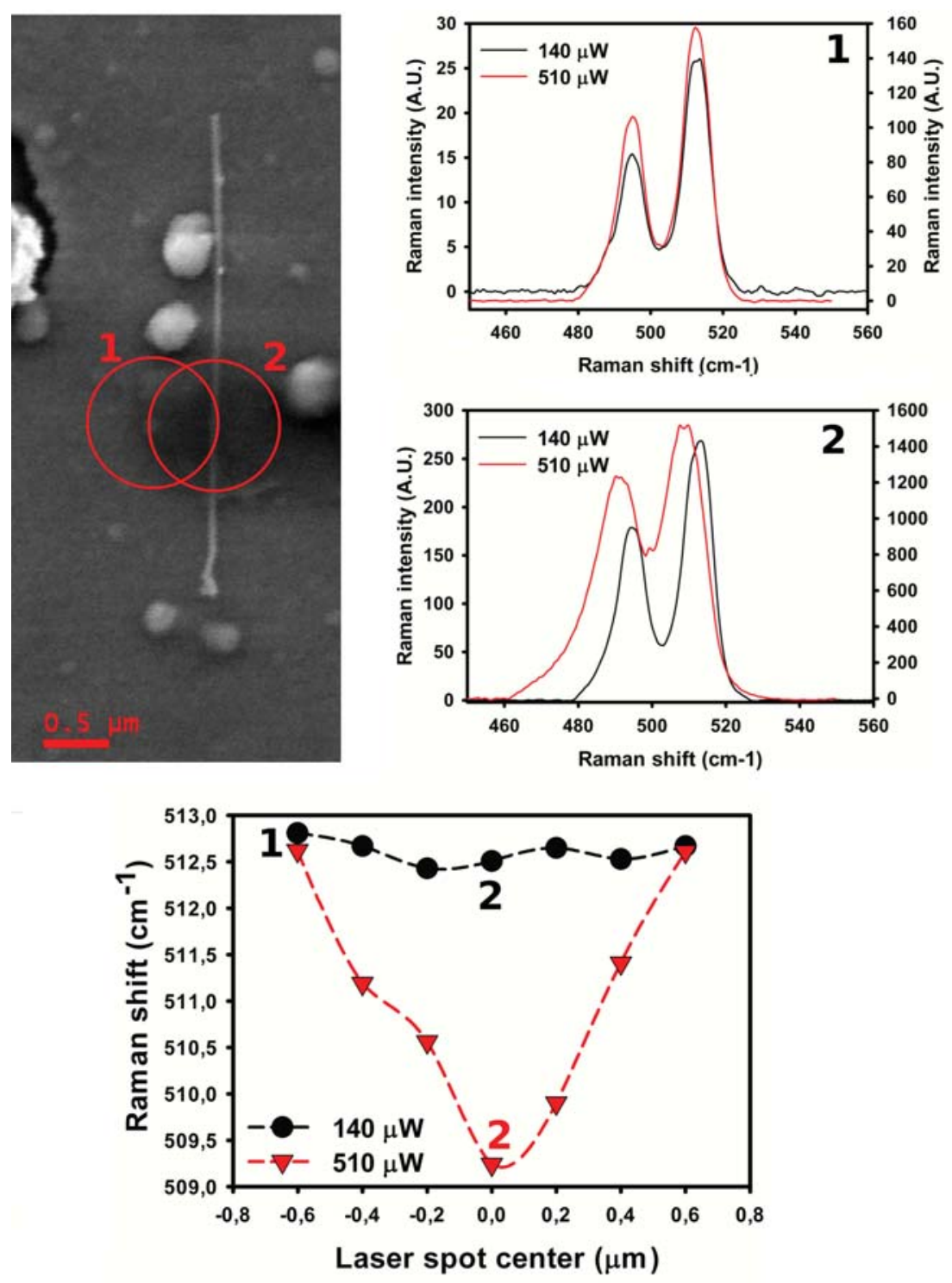

Figura 4.21: A) Espectros Raman adquiridos en el NW de $S i_{\sim 88} G e_{\sim 12}$ mostrado en la imagen SEM. Los espectros se han adquirido a para dos potencias de láser de Nd:YAG, $146 \mu \mathrm{W}$ y $500 \mu \mathrm{W}$ y el NW se encuentra depositado sobre un substrato de aluminio. En 1 se muestra como cuando el NW se se encuentra en el borde del área de iluminación del láser, los picos Si-Si coinciden en posición y forma para ambas potencias. En 2, el NW se encuentra en el centro del área de iluminación, y por tanto la intensidad es mucho mayor que en 1. Puede observarse como para la potencia más alta aparece un desplazamiento muy apreciable hacia bajas frecuencias. B) Posición del pico Si-Si en función de la posición del NW en el área de iluminación. Puede observarse como para la potencia más alta aparece un perfil de temperatura dominado por la distribución gaussiana de la potencia del láser. El perfil para la potencia más baja es independiente de la posición en el área, lo que indica que para esa potencia no se induce una temperatura apreciable en el NW, siendo óptima para la caracterización del NW. Nótese como aparece de nuevo el pico adyacente que se observaba en los espectros adquiridos en NWs de silicio. Esto será tratado en un sección posterior. 


\section{ESPECTROSCOPIA RAMAN EN NWS DE SILICIO Y SILICIO-GERMANIO}

en particular de los NWs silicio-germanio si se quiere realizar una caracterización composicional de los mismos. De no hacerlo, y debido a la fuerte dependencia de la temperatura inducida con la posición del NW en el área de iluminación, uno puede malinterpretar la señal Raman adquirida, de tal modo que se atribuya a un efecto de composición un desplazamiento de pico debido a la temperatura. Por otro lado, puede apreciarse en los espectros mostrados en la figura 4.21 que de nuevo aparece el pico adyacente visto para los NWs de silicio. Esto es un resultado muy relevante y será analizado posteriormente.

\subsubsection{Caracterización composicional de NWs de $S i_{1-X} G e_{X}$ por espectroscopia Raman}

En esta sección se van a analizar NWs de silicio-germanio crecidos bajo diferentes condiciones por nuestros colaboradores de la Universidad Politécnica de Madrid. Los NWs han sido fabricados utilizando el método VLS con oro-galio como precursor. El oro-galio se ha depositado mediante LPCVD y se ha recocido durante 30 minutos a $773 \mathrm{~K}$ para formar las nanopartículas precursoras de los NWs. Posteriormente se ha inyectado en la cámara de crecimiento $\mathrm{Si}_{2} \mathrm{H}_{6}$ y $\mathrm{GeH}_{4}$ a 400 mTorr de presión para generar los NWs de silicio-germanio. Las proporciones de estos gases se han variado de acuerdo a la tabla 4.1 para obtener distintas composiciones de NW, de tal modo que la composición media de las tres muestras disponibles de NWs son $S i_{\sim 91} G e_{\sim 9}, S i_{\sim 88} G e_{\sim 12}$ y $S i_{\sim 85} G e_{\sim 15}$. La determinación de estas composiciones se ha realizado mediante HRTEM+EDX por nuestros colaboradores, pudiéndose observar un análisis composicional a lo largo de un NW de la muestra $S i_{\sim 88} G e_{\sim 12}$ mediante EDX en la figura 4.22.

Como podemos observar en la figura 4.22, podemos esperar una ligera variación

\begin{tabular}{|c|c|c|c|c|c|}
\hline Composición (\%Ge) & $\mathrm{Si}_{2} \mathrm{H}_{6} \mathrm{sccm}$ & $\mathrm{GeH}_{4} \mathrm{sccm}$ & $\mathrm{H}_{2} \mathrm{sccm}$ & Temperatura (K) & Tiempo de crecimiento (s) \\
\hline$\sim 9 \%$ & 8 & 4 & 88 & 718 & 300 \\
\hline$\sim 12 \%$ & 8 & 8 & 84 & 703 & 300 \\
\hline$\sim 15 \%$ & 8 & 16 & 76 & 683 & 300 \\
\hline
\end{tabular}

Cuadro 4.1: Condiciones de crecimiento para cada una de los NWs de siliciogermanio analizados. 
de la composición del NW a los largo de su longitud, siendo la zona más próxima a la nanoparticula de oro-galio la más débil en germanio, hasta el punto de no contener germanio en las últimas decenas de nanometros donde se forma aprecia la formación de un codo en el NW.
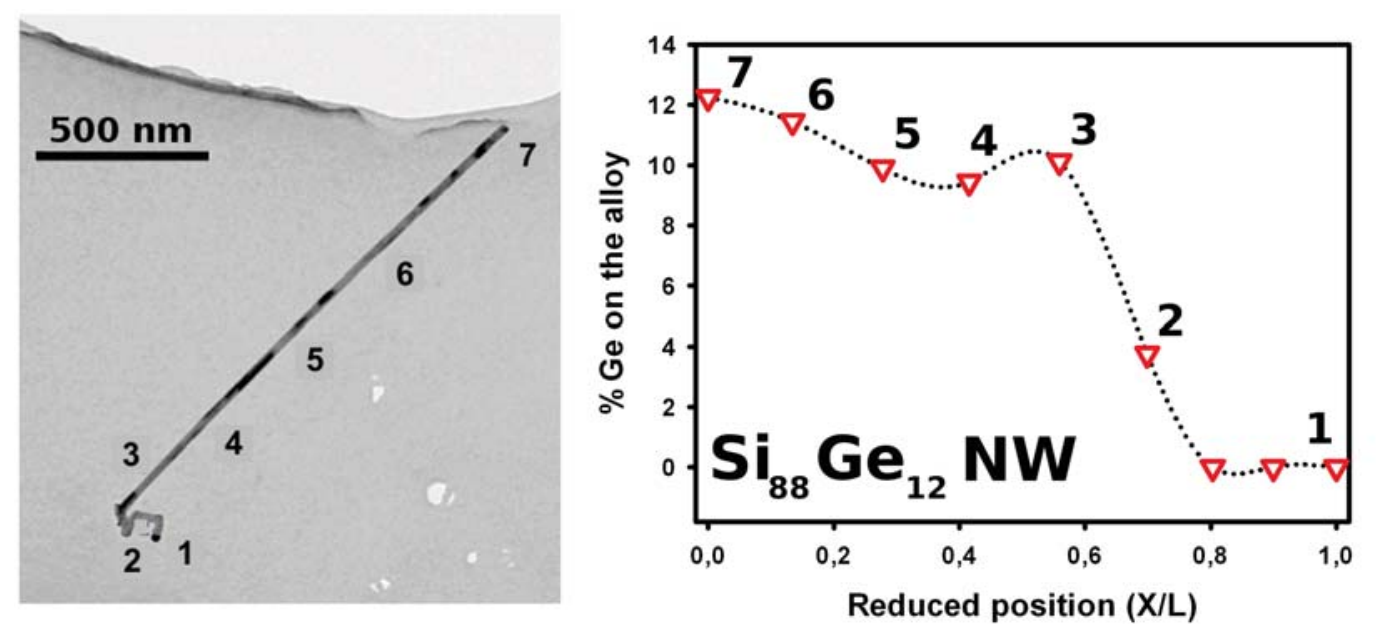

Figura 4.22: Imagen TEM de un NW silicio-germanio de la muestra caracterizada por una composición media de $\mathrm{Si}_{\sim 88} \mathrm{Ge}_{\sim 12}$. Puede observarse como la composición no es homogénea a lo largo del NW; la aleación es más débil en germanio cuanto más nos aproximamos a la bola de oro-galio. Debido al proceso de crecimiento la parte más próxima a la bola no contiene nada de germanio.

\section{Muestra con una composición media de $9 \%$ de germanio}

En la figura 4.23, se muestra un NW procedente de la muestra con una composición media del $9 \%$ de germanio. El NW esta aislado en su mayor parte, aunque aparece otro NW muy próximo y perpendicular a éste en uno de sus extremos. Sin embargo la existencia de este NW adyacente no afecta al análisis, ya que esta suficientemente separado como para poder desechar la parte en que ambos NWs son iluminados simultáneamente. La forma de determinar con precisión la posición que ocupan los NWs en el área iluminada sobre la que se adquiere el espectro Raman se realiza mediante un mapa Raman equivalente a los mostrados en la figura 4.6. El NW mostrado en la figura 4.23 tiene $90 \mathrm{~nm}$ de diámetro y $5.5 \mu \mathrm{m}$ de longitud, y está depositado sobre un substrato de aluminio. Se ha iluminado con el láser de 


\section{ESPECTROSCOPIA RAMAN EN NWS DE SILICIO Y SILICIO-GERMANIO}

Nd:YAG con una longitud de onda de $532.8 \mathrm{~nm}$, y se ha establecido la potencia óptima mediante el análisis de los cortes transversales. En este NW el contacto con el substrato es muy eficiente en términos de disipación, de tal modo que incluso para una potencia de $500 \mu \mathrm{W}$, no aparece un efecto de temperatura apreciable; obsérvese como los espectros adquiridos con el NW situado en el centro del área de iluminación son idénticos para $500 \mu \mathrm{W}$ y $50 \mu \mathrm{W}$ (Ver espectros mostrados en 4.6-1)). Con el fin de asegurar una completa eliminación del efecto de temperatura, uno podría pensar que la mejor opción es coger la menor potencia utilizada; sin embargo, los picos pertenecientes a los enlaces Si-Ge y Ge-Ge son tan débiles para está composición de la aleación, que al utilizar una potencia muy baja los picos son difíciles de caracterizar. Para solventar está dificultad, se ha escogido este NW de entre todos los depositados, ya que permite una caracterización a mayor potencia, sin que ello signifique comprometer la medida gracias a sus excelentes condiciones de disipación. En el espectro numerado como 1 en 4.23, puede apreciarse como éste es invariante frente a la potencia, evidenciando que no hay un efecto de temperatura apreciable; mientras tanto, en la zona ampliada a los picos $\mathrm{Si}-\mathrm{Ge}$ y $\mathrm{Ge}-\mathrm{Ge}$, se aprecia como el pico Ge-Ge aparece muy poco desplazado hacia bajas frecuencias, cuando lo esperado sería una aparición a más baja frecuencia para una composición tan pobre en germanio como esta. Por otro lado, es preciso advertir como aparece el pico adyacente a $\sim 495 \mathrm{~cm}^{-1}$, y además la intensidad del espectro Raman obtenido es mucho mayor de lo esperado, comportamientos ya observados en los NWs de silicio y que posteriormente trataremos. Como ya se ha dicho, la posición de los picos $\mathrm{Si}$-Si es constante a lo largo del corte transversal descrito en la figura 4.21 para cada punto de adquisición mostrado en 4.22, de tal modo que podemos asegurar que las posiciones de los picos solo van a depender de la composición que cada zona de iluminación del NW. En la figura 4.23-A), se represente el perfil de frecuencias para los picos Si-Si y Ge-Ge a lo largo del NW, pudiéndose observar como dicho perfil depende ligeramente de la zona del NW que este siendo iluminada, lo que refleja la existencia de una ligera inhomogéneidad en la composición del NW a lo largo de su longitud. La mayor parte del NW, analizada en los puntos 2,3 y 4 que mapean la parte central del NW, muestran una composición de entre el $\sim 8.5 \%$ y el $\sim 9 \%$, cuando se extrae su composición de la frecuencia a la que a 

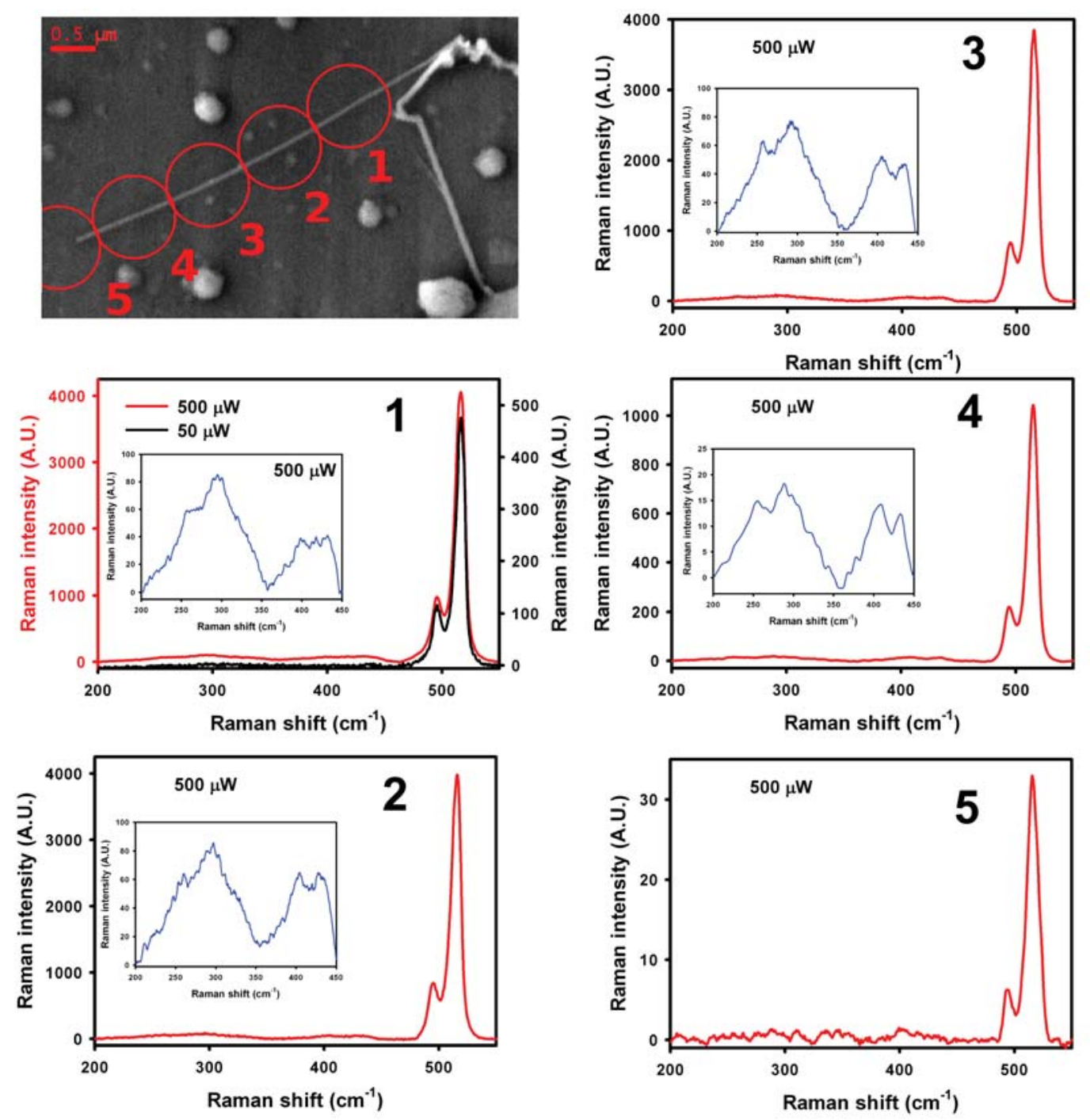

Figura 4.23: Imagen SEM del NW de silicio-germanio la muestra con una composición media del $12 \%$ de germanio. Las imágenes numeradas de 1 al 5 muestran los espectros obtenidos en los puntos señalados en la imagen SEM, separados aproximadamente $1 \mu \mathrm{m}$ para asegurar que se iluminan zonas independientes en cada paso. En 1 se muestran los espectros adquiridos a $500 \mu \mathrm{W}$ y a $50 \mu \mathrm{W}$, mostrando que el espectro no cambia al modificar la potencia, por lo que no se induce una temperatura apreciable en el NW con ninguna de las dos potencias. En azul aparece ampliada la ventana espectral correspondiente a los picos Ge-Ge y Si-Ge ya que su intensidad es muy débil para esta composición de la aleación. 


\section{ESPECTROSCOPIA RAMAN EN NWS DE SILICIO Y SILICIO-GERMANIO}

parece el pico $\mathrm{Si}-\mathrm{Si}$, en perfecto acuerdo con la composición media estimada para los NWs de esta muestra por HRTEM+EDX. La parte más cercana a la bola de oro-galio muestra una composición más débil en germanio, $\sim 7 \%$, un resultado también esperado dado que sabemos que la parte final del NW es más pobre en germanio. Por último, la composición en el punto 1 también muestra una menor composición en germanio, $\sim 7 \%$. La explicación de esta caída en la composición no pude establecerse tan claramente como cuando se ilumina la punta del NW, ya que en este caso existe un NW próximo a la zona de iluminación. Si bien se ha intentado adquirir el espectro en una zona en la que aparentemente no se ilumina el NW adyacente, distinguir categóricamente entre un efecto de composición y un efecto debido a la iluminación de 2 NWs puede resultar aventurado.

La composición determinada por la posición del pico Si-Si se corresponde perfectamente con a determinada por otros métodos para los NWs de esta muestra; sin embargo, cuando uno determina la composición mediante la posición del pico Ge-Ge el resultado es muy distinto. La composición calculada a través de las frecuencias mostradas en 4.24-A) para el Ge-Ge muestran que la aleación es muy rica en germanio y muy inhomogénea a lo largo del NW, variando entre el $\sim 75 \%$ y el $85 \%$ de germanio. En la misma zona ( 2 a 4 en figura 4.22), la composición obtenida mediante el pico Si-Si apenas oscilaba entre el $\sim 8.5 \%$ y el $\sim 9 \%$, coincidiendo con la composición media de la muestra; por tanto, la explicación mas probable nos indica que algo sucede en el pico Ge-Ge que falsea la medida en este NW.

\section{Muestra con una composición media de $12 \%$ de germanio}

Cuando se aumenta la cantidad de germanio en la aleación, los picos Ge-Ge y SiGe se van intensificando respecto al $\mathrm{Si}-\mathrm{Si}$, lo que permite que sean analizados de forma precisa incluso a baja potencia. En la figura 4.25 podemos observar la imagen SEM de un NW de silicio-germanio tomado de la muestra caracterizada por una composición media del $12 \%$ en germanio. Este NW esta completamente aislado y depositado en un substrato de aluminio. Tiene un diámetro de $\sim 90 \mathrm{~nm}$ y una longitud de $\sim 4 \mu \mathrm{m}$ y se ha iluminado con el láser de Nd:YAG, estableciéndose una potencia óptima de $146 \mu \mathrm{W}$, para la cual las posiciones del pico Si-Si en todos los cortes transversales son independientes de la posición del NW en el área de iluminación. Con el fin de obtener algo más de resolución en la determinación 


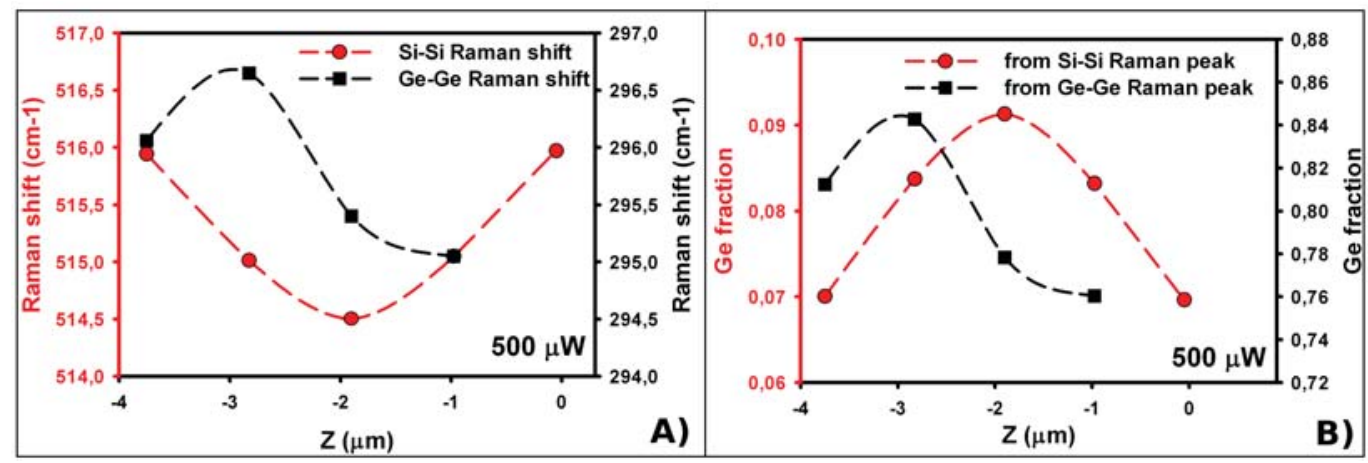

Figura 4.24: A) Perfiles de posición de los picos Si-Si y Ge-Ge en función del punto a los largo de la longitud del NW en el que se focaliza el haz láser. El pico Si-Si se lee en la escala de la izquierda (roja) y el Ge-Ge en la derecha (negra). Las lineas son únicamente una guía para el ojo. B) Composiciones obtenidas de las frecuencias mostradas en la figura A) para los picos Si-Si y Ge-Ge. Puede observarse la enorme discrepancia entre las composiciones obtenidas de cada pico. Nótese también la buena correspondencia obtenida entre la determinación de la composición mediante el pico Si-Si y la composición determinada para los NWs de esta muestra por EDX.

de la composición a lo largo del NW, cada punto de adquisición está separado del siguiente $600 \mathrm{~nm}$. Esto implica que hay una pequeña superposición en las partes iluminadas del NW entre puntos de adquisición, pero al ser una distancia mayor a la resolución lateral RL deducida en el capítulo 1, puede asumirse que se distingue perfectamente entre las zonas iluminadas. En la figura 4.25, aparecen diferentes espectros tomados en las zonas numeradas en la imagen SEM, pudiéndose observar a simple vista, como cuando se ilumina la zona donde está la bola de oro-galio existe una menor cantidad de germanio en la aleación (figura numerada como 1); además, los picos Ge-Ge y Si-Ge son mucho más débiles que los observados en el resto de espectros de la figura. Esto no es debido únicamente a estar iluminado con una menor potencia del láser, ya que el espectro 5 está iluminado de una forma similar (solo una parte del NW se encuentra dentro del área de iluminación) y aun así el pico Ge-Ge es más evidente. Otra cosa que llama la atención es que los picos Si-Ge y Ge-Ge aparecen más intensos que lo observado típicamente en aleaciones de silicio-germanio de una composición similar, siendo especialmente evidente en el espectro 4, donde se puede observar perfectamente el pico Ge-Ge con una posición y forma muy similar a la del germanio puro. En la figura 4.26-A), 


\section{ESPECTROSCOPIA RAMAN EN NWS DE SILICIO Y SILICIO-GERMANIO}
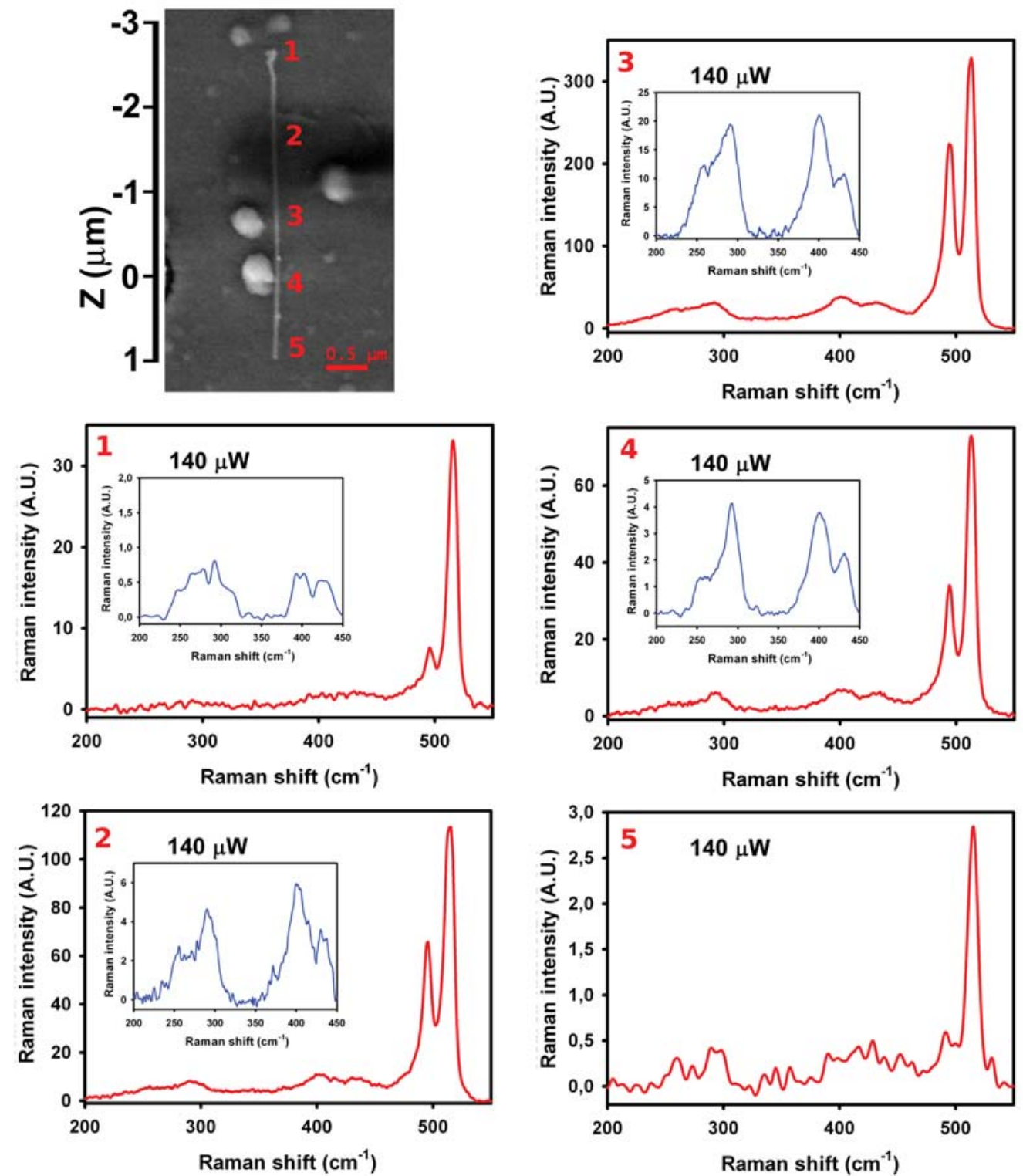

Figura 4.25: Imagen SEM de un NW de silicio-germanio de la muestra correspondiente a una composición del $12 \%$ de germanio. Los números muestran la zona iluminada en donde se han tomado los espectros numerados que aparecen a continuación. En azul aparece una representación en el rango espectral correspondiente a los picos Ge-Ge y Si-Ge para su mejor apreciación. 
se representan las posiciones de los picos representativos en función de la sección de NW que está siendo iluminada en cada caso. Resulta especialmente llamativo que los perfiles seguidos por las frecuencias para el pico Si-Si y Ge-Ge no parecen estar correlacionados; sin embargo, sabemos que en aleaciones volúmicas de silicio-germanio ambos están anticorrelacionados, tal y como aparece en la figura 4.19. Cuando se extraen las composiciones de estas frecuencias para los picos $\mathrm{Si}-\mathrm{Si}$ y Ge-Ge, podemos observar como la composición calculada a través del pico Si-Si se corresponde muy bien con la composición media determinada para esta muestra por EDX, obteniendo un porcentaje de germanio de $\sim 12 \%$ a lo largo de todo el NW, salvo cuando nos aproximamos a la zona donde está la bola de oro-galio, donde el NW se empobrece en germanio. Cuando el análisis composicional se realiza a través del pico Ge-Ge, se obtienen unos porcentajes de germanio en la aleación que oscilan entre el $\sim 59 \%$ y el $\sim 73 \%$. Estos valores tan elevados no son compatibles con lo determinado a través de pico $\mathrm{Si}-\mathrm{Si}$, ni tampoco con la forma del espectro obtenido. Una aleación con una composición del $\sim 73 \%$ debería resultar en un espectro con un pico $\mathrm{Ge}-\mathrm{Ge}$ mucho más intensos que el pico $\mathrm{Si}-\mathrm{Si}$, sin embargo en los espectros de la figura 4.25 el pico Ge-Ge es muy débil. Por otro lado, si comparamos el perfil de composición obtenido con el pico Si-Si con el determinado por EDX, mostrado en la figura 4.22 para un NW procedente de la misma muestra que este, podemos ver como la composición determinada por ambos métodos es muy similar. Esto nos indica que lo observado en el pico $\mathrm{Si}-\mathrm{Si}$ es correcto, pero que de nuevo, algo sucede con el pico Ge-Ge que falsea su composición.

\section{Muestra con una composición media de $15 \%$ de germanio}

Hasta el momento hemos observado como para los NWs de silicio-germanio de dos composiciones diferentes, la espectroscopia Raman se muestra como una herramienta muy potente para determinar la composición a lo largo del NW, siempre y cuando la composición se extraiga de la frecuencia del pico Si-Si. En la figura 4.27 se muestra la imagen SEM de un NW de una composición diferente, más rica en germanio que las anteriores. La composición media para la muestra de la que procede se ha determinado como $S i_{85} G e_{15}$ por EDX, y el NW se encuentra depositado en un substrato de aluminio. El diámetro del NW es muy similar al de 

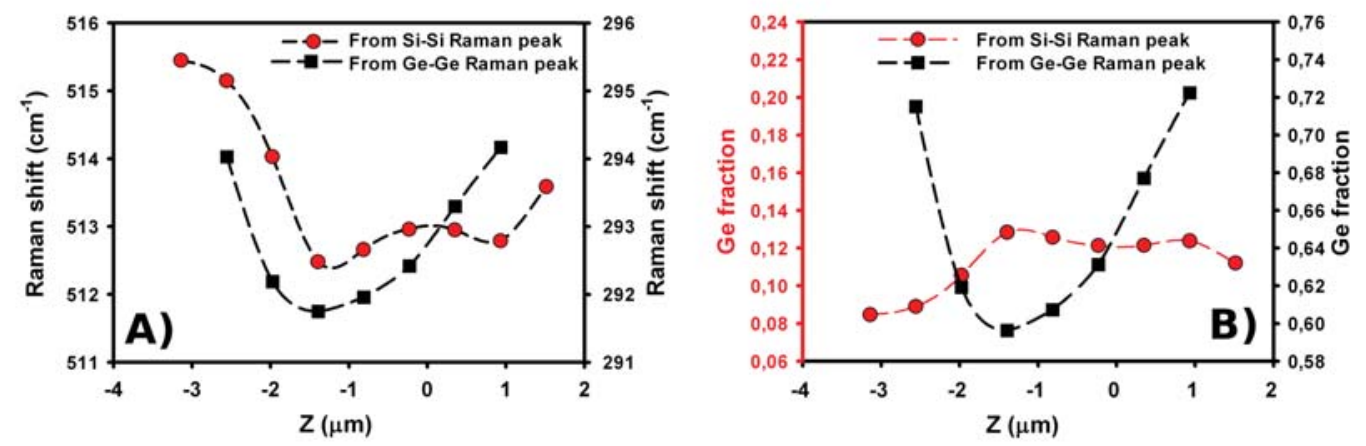

Figura 4.26: A) Frecuencia a la que aparecen los picos Si-Si (círculos rojos) y GeGe (cuadrados negros) en función de la posición a la que se centra el spot láser a lo largo del NW. Nótese la falta de correlación evidente entre las posiciones de ambos picos. B) Perfil de composición del NW a lo largo de su longitud calculada a través de las frecuencias de los picos Si-Si (rojo) y Ge-Ge (negro). Puede observarse la gran discrepancia entre las composiciones determinadas a través de cada pico. En ambas figuras el pico Si-Si se lee en la escala izquierda (roja) y el Ge-Ge en la derecha.

los anteriores NWs analizados, i.e. $\sim 90 \mathrm{~nm}$; mientras que su longitud es algo menor a $4.5 \mu \mathrm{m}$. Para eliminar cualquier efecto de temperatura que pudiese falsear el análisis de los espectros se ha tenido que bajar la potencia del láser de Nd:YAG hasta los $50 \mu \mathrm{W}$, obteniéndose con esta potencia unos espectros completamente independientes de la posición del NW el área de iluminación para todos los cortes transversales realizados a lo largo del NW. Este NW tiene la particularidad de que no ha sido seccionado limpiamente del substrato, ya que en una de sus puntas conserva parte de del substrato donde ha crecido. Por tanto, el espectro Raman obtenido de la punta contaminada por el substrato no se tiene en cuenta para el análisis. En los espectro numerados según la zona iluminada, llama especialmente la atención la gran intensidad relativa al pico Si-Si con la que aparece el pico $\mathrm{Ge}$ Ge en algunos espectros (1,5), y lo inhomogénea que es dicha intensidad relativa a lo largo del NW. Además, la posición y la forma del pico corresponden más al espectro Raman del germanio puro que al débil pico que uno esperaría encontrar para una composición tan pobre en germanio. Por otro lado llama la atención que en este NW no aparece el pico adyacente a $\sim 495 \mathrm{~cm}^{-1}$. Este resultado no es único de este NW, ya que en los dos NWs mostrados en la figura 4.20, que también pertenecían a esta muestra, tampoco aparecía este pico adyacente. Cuando uno ob- 

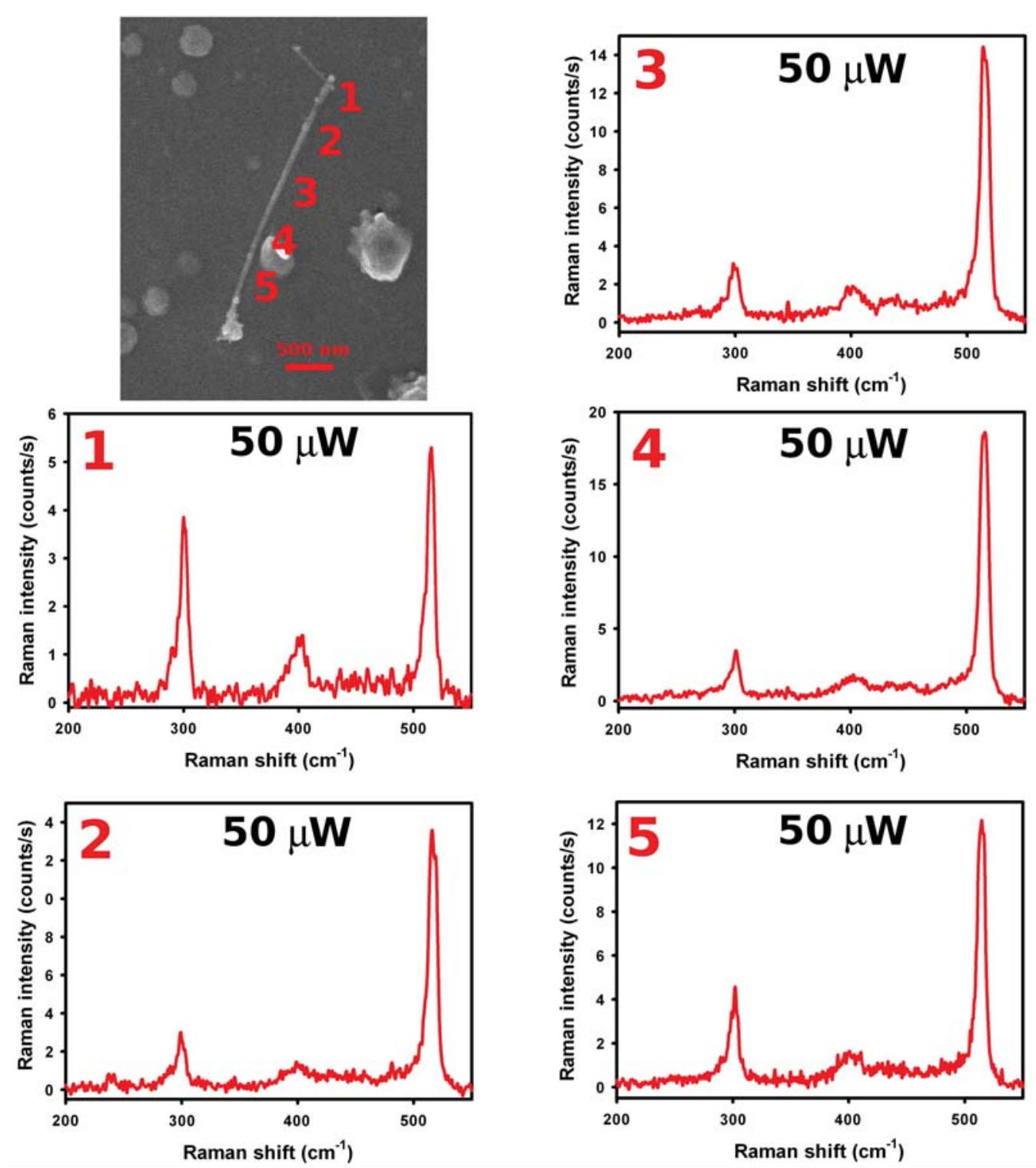

Figura 4.27: Imagen SEM de un NW de silicio-germanio procedente de la muestra con una composición media del $15 \%$ de germanio. El NW se encuentra depositado en un substrato de aluminio, tiene unas dimensiones de $90 \mathrm{~nm}$ de diámetro en su parte más gruesa y $\sim 80 \mathrm{~nm}$ en su parte más fina, por otro lado su longitud es de $\sim 4.5$ $\mu \mathrm{m}$. En los espectros obtenidos en las distintas partes del NW podemos observar la gran intensidad relativa del pico Ge-Ge respecto al pico Si-Si y la inhomogéneidad de dicha intensidad relativa a lo largo del NW. Por otro lado llama la atención que no esté presente el pico suplementario a $\sim 495 \mathrm{~cm}^{-1}$. 


\section{ESPECTROSCOPIA RAMAN EN NWS DE SILICIO Y SILICIO-GERMANIO}

serva las frecuencias a las que aparecen los picos $\mathrm{Si}-\mathrm{Si}$ y $\mathrm{Ge}-\mathrm{Ge}$ en en este NW en función de la zona del NW que es iluminada (figura 4.28-A), se observa que la intensidad relativa del pico Ge-Ge frente al Si-Si no parece estar correlacionada con la posición del pico $\mathrm{Si}$-Si; la posición menos desplazada corresponde a la zona de la bola de oro-galio, donde la intensidad relativa del pico Ge-Ge era más alta. Por otro lado, el pico Ge-Ge aparece a una frecuencia más alta cuando se ilumina el centro del NW, donde la intensidad relativa del Ge-Ge es menor. Cuando nos fijamos en las composiciones extraídas de las frecuencias (figura 4.28-B)), podemos observar como la zona menos rica en germanio corresponde a la zona de la punta que tiene la bola de oro-galio, tal y como se espera del crecimiento de estos NWs [65]. La composición calculada a través del pico Si-Si apenas alcanza el $13 \%$ en su punto máximo, mientras que sabemos que debería estar cerca del $15 \%$. Por otro lado, la composición determinada por el pico Ge-Ge muestra que la composición es próxima al $100 \%$ de germanio. Uno podría pensar que estos resultados tan extraños
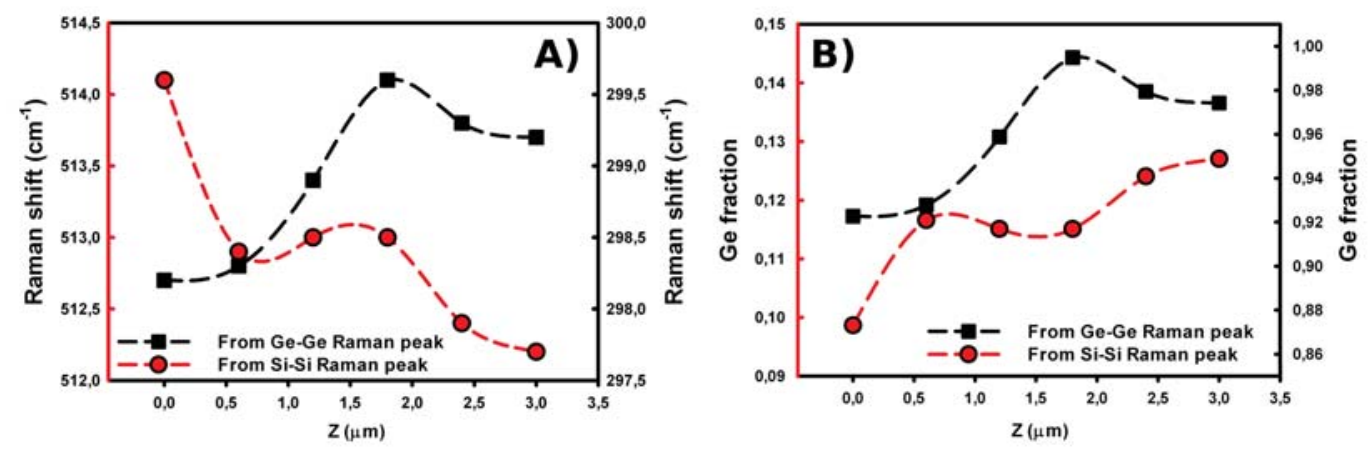

Figura 4.28: A) Frecuencias a las que aparecen los picos $\mathrm{Si}-\mathrm{Si}$ (círculos rojos) y GeGe (cuadrados negros) en función del punto donde se centra el haz láser a lo largo del NW. B) Composiciones determinadas a través de las frecuencias mostradas en A). Pude observarse como la composición determinada por el pico GE-Ge muestra que tenemos un NW de germanio puro, mientras que la determinada por el Si-Si muestra una composición menor a la determinada por EDX para esta muestra.

corresponden exclusivamente al NW mostrado en 4.27. Sin embargo, para todas las composiciones se han analizado distintos NWs, obteniendo siempre el mismo comportamiento en cada caso, seleccionándose para este trabajo los NWs más limpios y que ofrecen unos resultados más claros. En la figura 4.29 se muestra un grupo 
de NWs pertenecientes a la muestra con un $15 \%$ de germanio y que pueden ser analizados individualmente. Podemos observar que tanto en el NW de $\sim 60 \mathrm{~nm}$, al que corresponde el espectro 1, como para el NW de $\sim 90 \mathrm{~nm}$ al que corresponden los espectros 2 y 3 , se observa un pico Ge-Ge más intenso que lo correspondiente a esta composición. Además el pico secundario, que si que aparece en estos NWs, es muy débil. Si nos fijamos en las composiciones extraídas de estos NWs, pode-
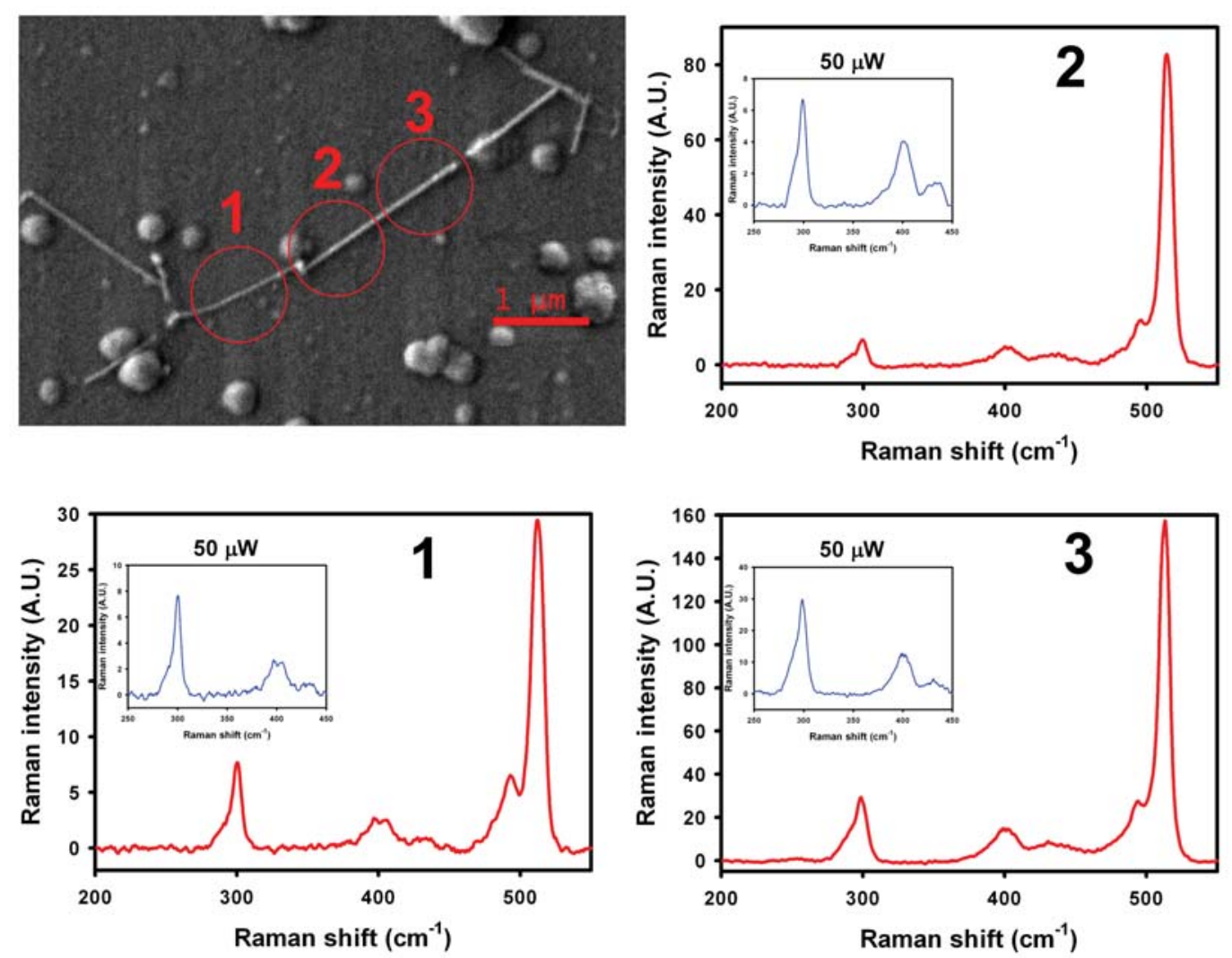

Figura 4.29: Imagen SEM de un grupo de NWs de silicio-germanio pertenecientes a la muestra caracterizada por un $15 \%$ de germanio. Puede observarse como los NWs están alineados longitudinalmente y suficientemente separados como para poder analizar por separado sus espectros. Nótese como el pico Ge-Ge aparece a una frecuencia muy próxima al germanio puro y con una intensidad muy elevada para la poca cantidad de germanio presente en la aleación.

mos observar como de nuevo aparece una composición de entorno el 12\%-13\% en las zonas medias de los NWs cuando se analiza el pico Si-Si, menores por tanto que la composición esperada del 15\% (El punto 2 ilumina las zonas próximas a 


\section{ESPECTROSCOPIA RAMAN EN NWS DE SILICIO Y SILICIO-GERMANIO}

la bola de oro-galio en ambos NWs, por lo que su composición es más pobre en germanio). Por otro lado, ambos NWs muestran una composición cercana al $100 \%$ de germanio cuando se analiza el pico Ge-Ge (ver figura 4.30-B)). Mostrando que este comportamiento es característico de la muestra y no un evento esporádico. Por

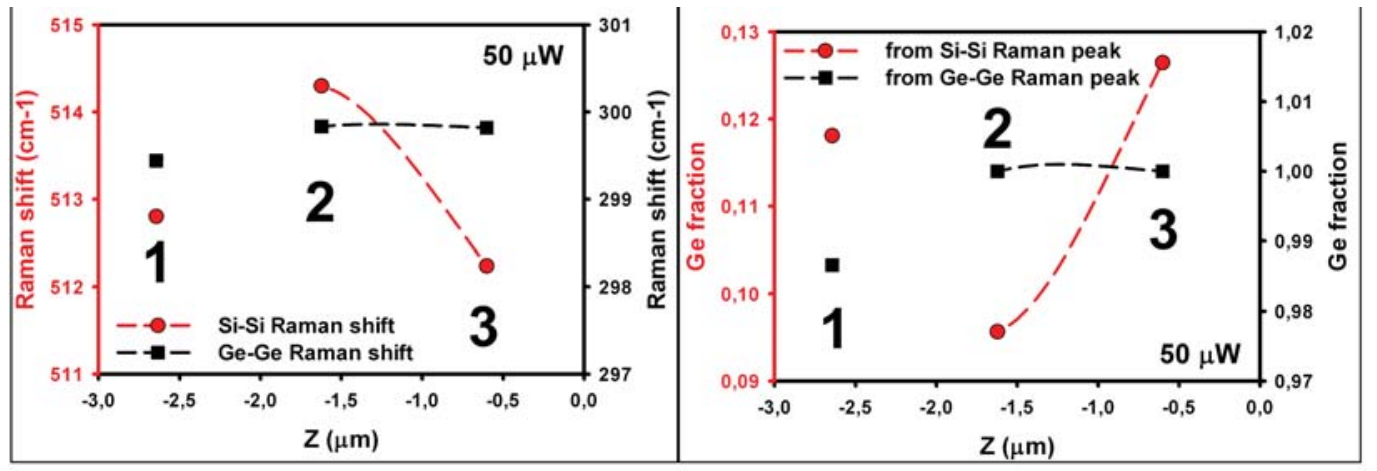

Figura 4.30: A) Frecuencias a las que aparece los picos Si-Si y Ge-Ge para cada región iluminada mostrada en 4.19. Composiciones calculadas a través de las frecuencias mostradas en A)

tanto, puede concluirse que el análisis Raman de estos NWs atribuye una menor proporción de germanio que la esperada cuando se caracteriza a través del pico Si$\mathrm{Si}$, mientras que cuando se caracteriza por el Ge-Ge lo que se obtiene es una señal perteneciente a una aleación donde prácticamente todo es germanio.

\subsubsection{Anomalías encontradas al analizar el espectro Raman de los NWs de $S i_{1-X} G e_{X}$.}

Los espectros Raman obtenidos de los distintos NWs de silicio-germanio analizados encierran bastantes resultados que no se corresponden con lo que uno podría esperar. Dos de estos resultados ya habían sido advertidos cuando se analizaron los NWs de silicio:el primero el la gran intensidad de señal Raman que se obtiene al iluminar los NWs, y el segundo la aparición de un pico suplementario entorno a los $495 \mathrm{~cm}^{-1}$. Además de estos efectos, los NWs de silicio-germanio incorporan un efecto anómalo más, ya que existe una gran discrepancia entre la determinación de la composición a través del pico correspondiente a los enlaces $\mathrm{Si}$-Si y el pico 
correspondiente a los enlaces Ge-Ge. En esta sección se analizaran las posibles causas que justifican la aparición de estos resultados anómalos.

\subsubsection{Segregación del Germanio en la aleación y existencia de una coraza rica en Ge}

Hemos podido observar como en mayor o menor medida, todos los NWs de siliciogermanio de nuestras muestras tienen un espectro Raman con una forma que no se corresponde con el espectro Raman esperado para una aleación de $S i_{1-X} G e_{X}$ volúmica de igual composición.

En las aleaciones de silicio-germanio, bien por oxidación o bien por las condiciones de crecimiento, puede aparecer una segregación del germanio de la aleación hacia las capas externas de la muestra [67,69-71]. En la figura 4.31 se muestra un perfil transversal de composiciones de un NW de $\sim 55 \mathrm{~nm}$ de diámetro medido por EDX y tomado de la muestra caracterizada por una composición media del $12 \%$. Puede observarse como la cantidad de germanio en la aleación no es constante a lo largo del corte, apareciendo unas zonas enriquecidas en germanio en los bordes del NW. En las figura 4.31 se muestra también una representación esquemática de la estructura del NW; los NWs, por efecto de la oxidación/condiciones-de-crecimiento, tienen una capa muy rica de germanio en la zona cercana a la superficie (apenas unos nanometros), que disminuye rápidamente al penetrar en el NW, de tal modo que el NW no es homogéneo en composición, si no que muestran una estructura que recuerda levemente a un NW "core - shell" [67]. Además, esta estructura no es simétrica, pudiendo ser más ricas en germanio determinadas partes aisladas de la superficie en una misma sección del NW. Como podemos observar en la figura 4.32, el espectro que obtenemos en los NWs de la muestra $S i_{85} G e_{15}$ es muy similar al que se obtiene de un NW de silicio-germanio core-shell en el que la coraza exterior solo está compuesta por germanio [67]. Por tanto, la distribución inhomogénea de germanio dentro de nuestros NW es la causante de que veamos tan intenso el pico Ge-Ge y por que se falsea una aleación tan rica en germanio cuando determinamos la composición mediante el pico Ge-Ge. Sin embargo, el espectro Raman procedente de la referencia 37 y mostrado en 4.32-A) corresponde a un NW con una coraza de $\sim 40 \mathrm{~nm}$ de germanio puro y un núcleo de silicio-germanio muy rico en silicio y de $\sim 20 \mathrm{~nm}$; mientras que el NW mostrado en la figura 4.32-A), dada 

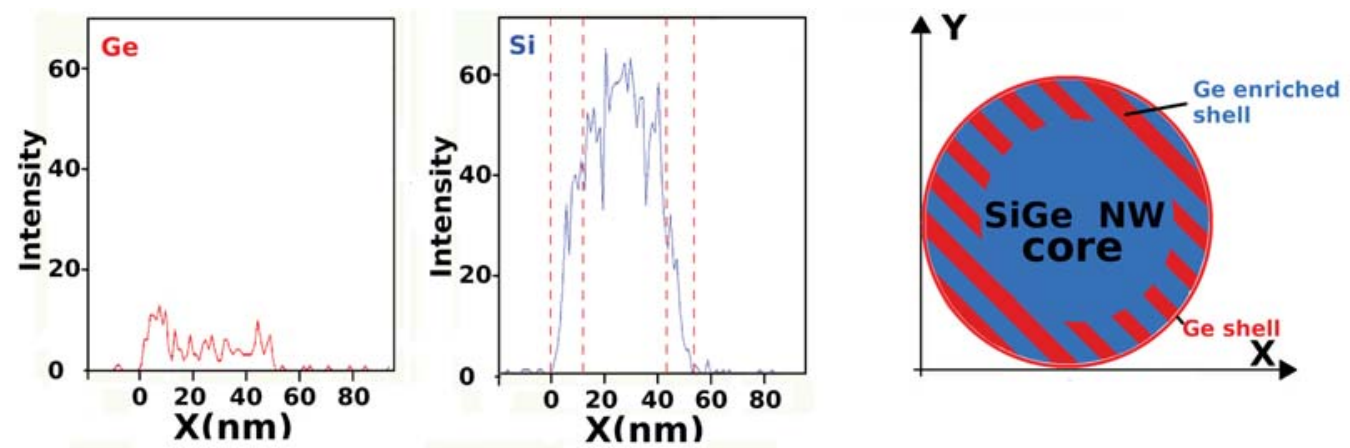

Figura 4.31: Proporciones de germanio y de silicio en un NW perteneciente a la muestra caracterizada por una composición media del $12 \%$ de germanio. Puede observarse como el germanio no está distribuido de forma homogénea, sino que aparecen zonas ricas en germanio en la zona cercana a la superficie (ver esquema de la estructura core-shell que presentan los NWs).

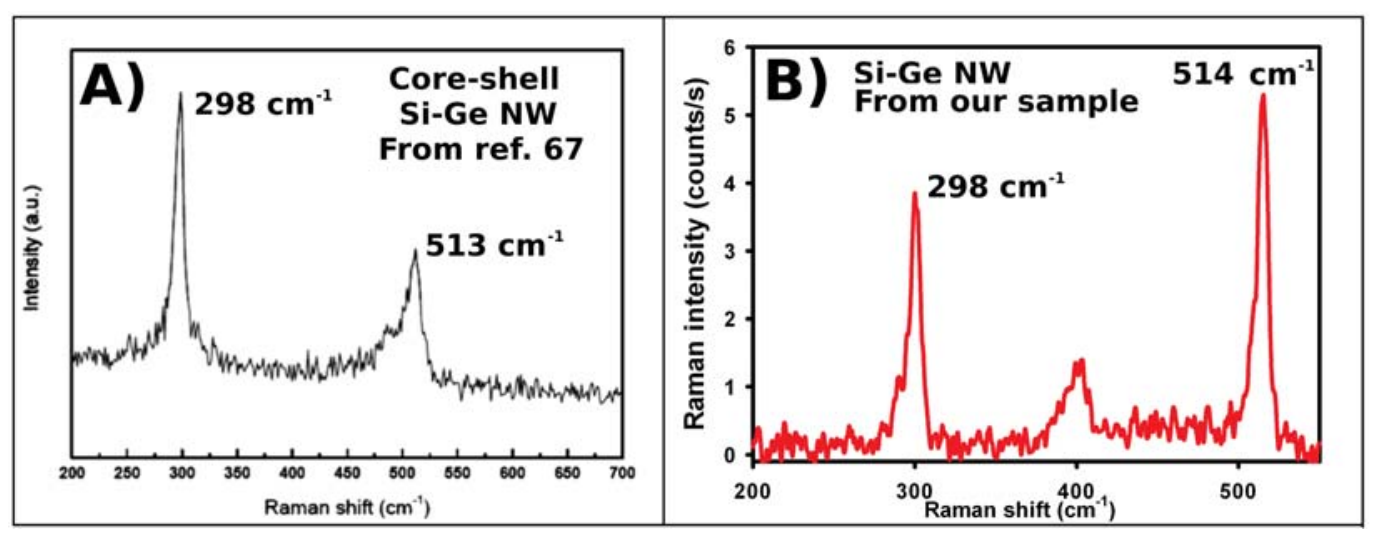

Figura 4.32: A) Espectro Raman procedente de la referencia [67] adquirido sobre un NW de silicio-germanio con estructura core-shell donde la corteza (shell) tiene cerca de $40 \mathrm{~nm}$ de grosor y esta compuesta únicamente por germanio, mientras que el núcleo (core) tiene menos de $20 \mathrm{~nm}$ y está compuesto por una aleación de silicio-germanio muy rica en silicio. B) Espectro Raman adquirido en un NW de la muestra $S i_{85} G e_{15}$. Nótese la similitud entre ambos espectros pese a las enormes diferencias estructurales y composicionales que existen entre ambos 
su composición pobre en germanio (15\%), de tener una coraza de germanio puro, ésta no podría ser mayor a unos pocos nanométros. Por tanto, estamos viendo una contribución muy grande al espectro Raman procedente de un volumen de material muy pequeño en comparación con el resto del volumen del NW, y localizado en la superficie del NW. Este mismo efecto puede comprobarse en la figura 4.33A), donde podemos ver como es el espectro obtenido en una lamina de $S i_{88} G e_{12}$ adquirido con el láser de Nd:YAG. Este espectro muestra que la vibración correspondiente al pico Ge-Ge es muy débil y aparece entorno a $283 \mathrm{~cm}^{-1}$. Sin embargo, en los espectros obtenidos para un NW con la misma composición (ver figura 4.25), el pico correspondiente al Ge-Ge tiene una mayor intensidad y además aparece por encima de $292 \mathrm{~cm}^{-1}$, frecuencia que corresponde a una composición de al menos un $60 \%$ de germanio. En la figura 4.33-A) se muestra el espectro obtenido de una lamina de $S i_{40} G e_{60}$ con el láser de Nd:YAG. Este espectro no guarda ningún parecido con lo observado en la figura 4.25, sin embargo si se combinan los espectros de ambas laminas, dando un mayor peso a la señal obtenida del $S i_{88} G e_{12}$, podemos observar como se obtiene un espectro muy similar a lo que obtenemos en el NW (Figura 4.33.B) ). Por tanto, todo sucede como si estuviéramos viendo la superposición de dos contribuciones, una procedente del núcleo del NW, que se corresponde a una composición más cercana a la composición característica de la muestra, y otra procedente de la superficie que es mucho más rica en germanio por el efecto de segregación de este elemento hacia la superficie. A tenor de lo visto en los espectros adquiridos en cada muestra, cuanto mayor es la cantidad de germanio en la aleación, mayor es la cantidad de germanio que se segrega, y más cantidad de germanio puro aparece en la superficie. En la figura 4.33, puede observarse como los espectros obtenidos en diferentes zonas a lo largo del NW no tienen la misma forma, lo que implica que la segregación del germanio es muy inhomogénea a lo largo del NW (Ver figura 4.33 espectros identificados con letras). Por otro lado, cuando se hace un corte transversal, i.e. el NW ocupa distintas posiciones dentro del área de iluminación, básicamente se está iluminando la misma sección del NW. Esto implica que salvo por la intensidad de la señal global y una vez eliminados efectos de temperatura, los espectros obtenidos deben ser idénticos con independencia de la posición que ocupe el NW dentro del área de iluminación; sin embargo, mientras 


\section{ESPECTROSCOPIA RAMAN EN NWS DE SILICIO Y SILICIO-GERMANIO}

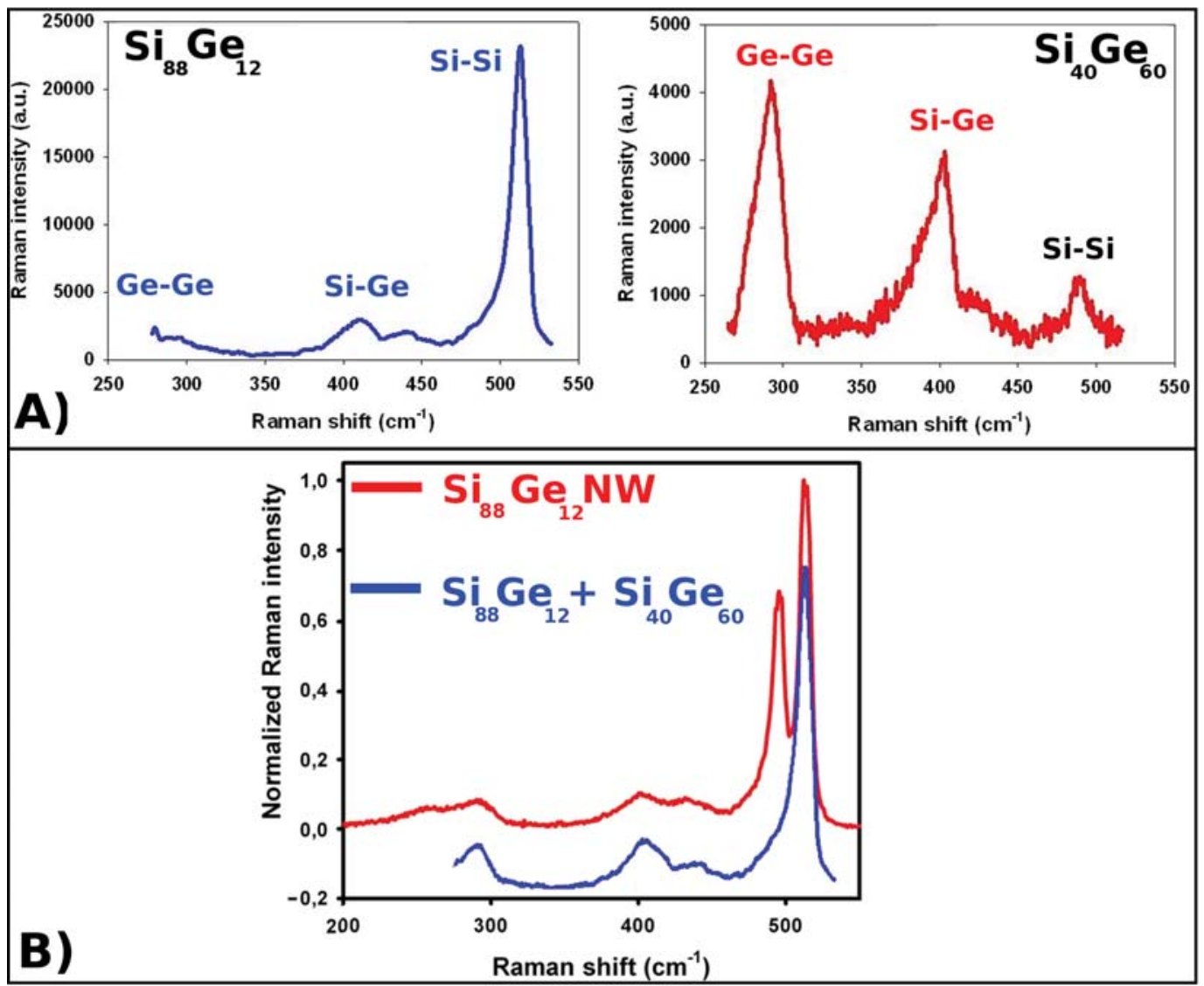

Figura 4.33: A) Espectros Raman obtenidos en laminas de silicio-germanio con un $12 \%$ y un $60 \%$ de germanio respectivamente. Puede observarse como ninguna de las dos se corresponde con los espectros obtenidos en los NWs con un porcentaje de germanio determinado por el pico Si-Si del $12 \%$ y del $60 \%$ mediante el pico Ge-Ge. B) Comparación del espectro obtenido para el NW de la figura 4.25 y del espectro resultante de dos contribuciones, una con un $12 \%$ de germanio y otra con un $60 \%$ de germanio. Puede observarse la similitud de este espectro con el obtenido del NW pese a la brusquedad de la aproximación, ya que el peso dado a las señales es aproximado. 
que la posición del pico Si-Si se mantiene, la posición del pico Ge-Ge y su intensidad relativa frente al pico Si-Si dependen fuertemente de la posición que ocupa el NW en el área de iluminación (Figura 4.33 espectros numerados). El origen de estas anomalías solo puede ser explicado si la intensidad Raman procedente de la superficie de los NWs está contribuyendo de una forma muy intensa a la señal Raman final, y por tanto tiene que existir un efecto que magnifica la señal procedente de esta zona de los NWs. La explicación a este fenómeno va a estar relacionada con la distribución del campo electromagnético en el interior de los NWs cuando son iluminados por el haz láser, pero su explicación detallada no puede adelantarse hasta que no se estudie en profundidad la interacción láser-NW-substrato vista desde un punto de vista electromagnético en el capítulo 5.
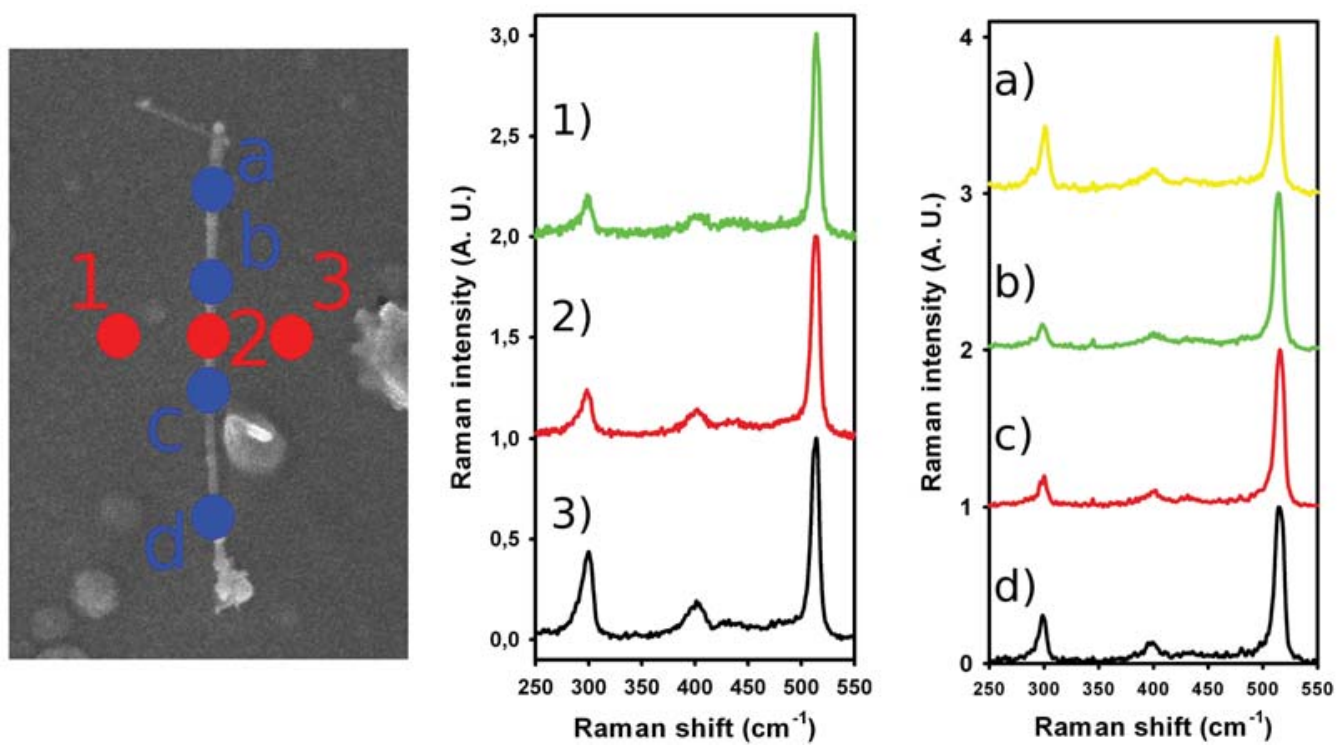

Figura 4.34: Variación de los espectros Raman obtenidos cuando el haz láser barre transversalmente un NW de $S i_{85} G e_{15}$ (números) y cunado el barrido se hace longitudinalmente (letras). Nótese la diferencia en las intensidades relativas del pico Ge-Ge frente al Si-Si en el corte transversal.

\subsubsection{Pico adyacente localizado a $\sim 495 \mathrm{~cm}^{-1}$}

En todos los espectros Raman adquiridos en los NWs de silicio-germanio pertenecientes a las muestras con un $9 \%$ y $12 \%$ de germanio, y raramente en NWs de 


\section{ESPECTROSCOPIA RAMAN EN NWS DE SILICIO Y SILICIO-GERMANIO}

la muestra con $15 \%$ de germanio, puede apreciarse la aparición de un pico adyacente muy similar al ya reportado en los NWs de silicio. La aparición de este pico en el espectro Raman de los NWs de silicio había sido atribuida a diversas causas [9, 34 - 37], siendo la más común la atribución de este pico suplementario a la presencia de politipos hexagonales en el NW [34,36,37]. Dado que todos los NWs de silicio-germanio han sido crecidos con oro-galio como catalizador, su estructura cristalina es muy buena, y no se ha observado en ellos la presencia masiva de estos politipos a lo largo del NW, salvo en una zona próxima a la partícula de oro-galio donde el NW está compuesto íntegramente por silicio [65](ver imagen 4.22, zonas 1 y 2 de la imagen TEM). Por otro parte, el pico Raman correspondiente al politipo $2 \mathrm{H}$ del silicio tiene que ser sensible a la cantidad de germanio en la aleación por la misma razón que lo es el pico correspondiente al silicio cúbico, el germanio modifica el parámetro de red causando un descenso a bajas energías en los enlaces Si-Si. Sin embargo, y como podemos observar en la figura 4.34-A), el pico adyacente aparece en el espectro Raman de los NWs de silicio-germanio analizados a $\sim 495 \mathrm{~cm}^{-1}$, con independencia de la cantidad de germanio que exista en la aleación. En la figura 4.34-B) se puede apreciar como el pico Raman de los enlaces Si-Si se desplaza entre $\sim 4$ y $\sim 7 \mathrm{~cm}_{-1}$ por el efecto de la aleación respecto a la frecuencia a la que aparece en NWs de silicio, mientras que el pico adyacente no muestra una variación relevante. Por tanto, la atribución de este pico a la presencia de politipos en el NW no parece justificable en nuestro caso. En la figura 4.35, se puede apreciar como depende la posición del pico suplementario con la posición que ocupa el NW de silicio-germanio en el área de iluminación para dos potencias del láser Nd:YAG diferentes. Podemos comprobar como existe una correlación entre los desplazamientos de las frecuencias por efecto de temperatura en el pico Si-Si y los desplazamientos que aparecen en el pico adyacente por el mismo motivo. Por tanto, dado que ambos picos tienen la misma dependencia con la temperatura, parece que ambos picos proceden de los enlaces $\mathrm{Si}$-Si presentes en la aleación. Si el pico 495 está causado por los enlaces Si-Si en el interior del NW, en las zonas más ricas en silicio del NW debería aparecer más intenso que en las zonas donde hay una mayor presencia de germanio. Por tanto, uno podría esperar que en la punta del NW, donde hay una mayor cantidad de silicio, la intensidad relativa del pico 495 frente al del Si-Si sea máxima. El resultado de este experimento se muestra 


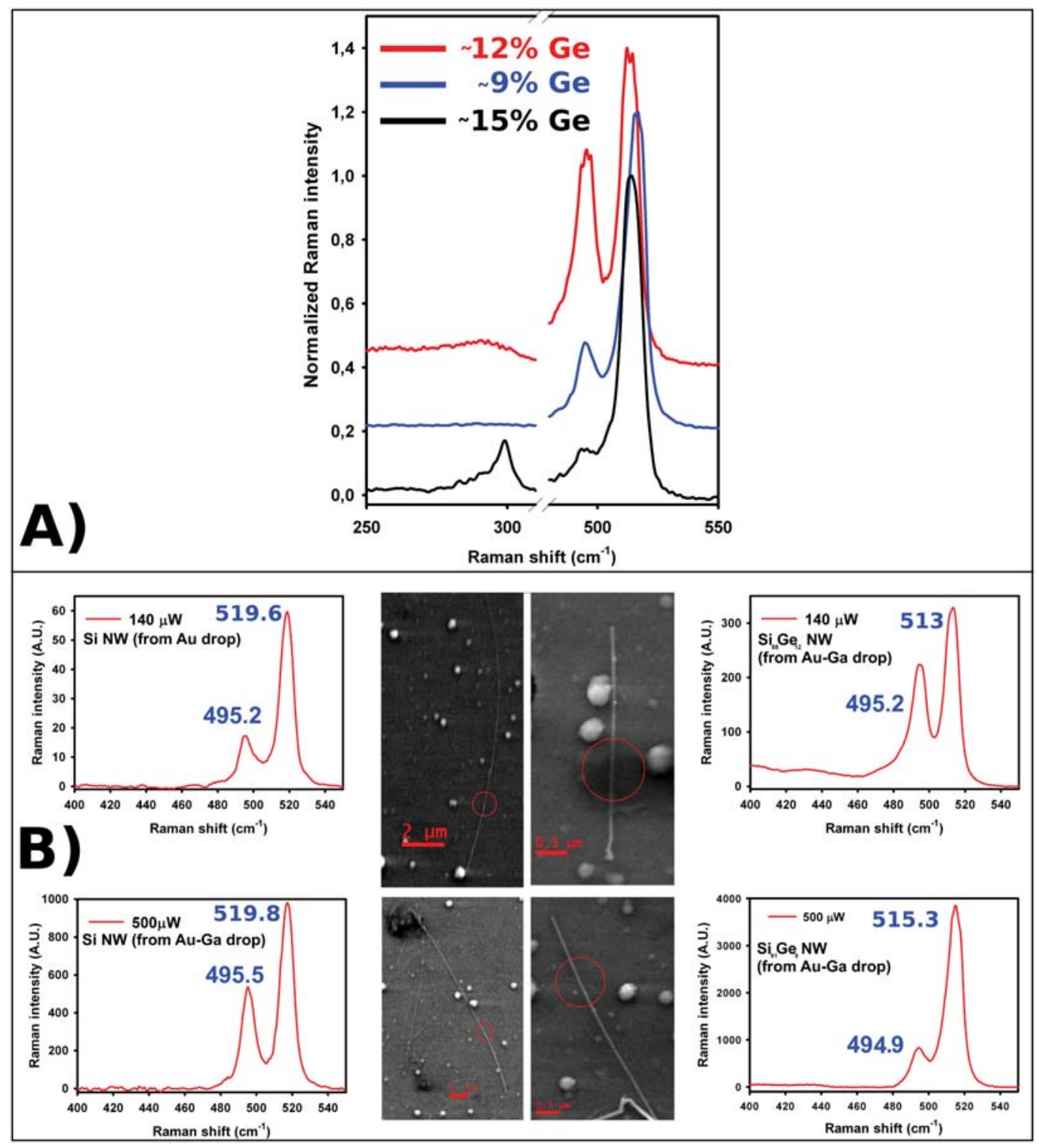

Figura 4.35: A) Espectros Raman correspondientes a tres NWs de silicio-germanio pertenecientes a muestras con tres proporciones de germanio en la aleación diferentes. Puede observarse como el pico suplementario aparece en la misma posición en cada caso $\left(495 \mathrm{~cm}^{-1}\right)$. B) Espectros Raman obtenidos en los NWs de silicio y siliciogermanio mostrados en las imágenes SEM. Nótese como las posiciones de las frecuencias de los picos Si-Si se desplazan en función de la composición con respecto a los picos obtenidos los NWs de silicio, mientras que los picos suplementarios se muestran invariantes a la existencia de germanio 


\section{ESPECTROSCOPIA RAMAN EN NWS DE SILICIO Y SILICIO-GERMANIO}
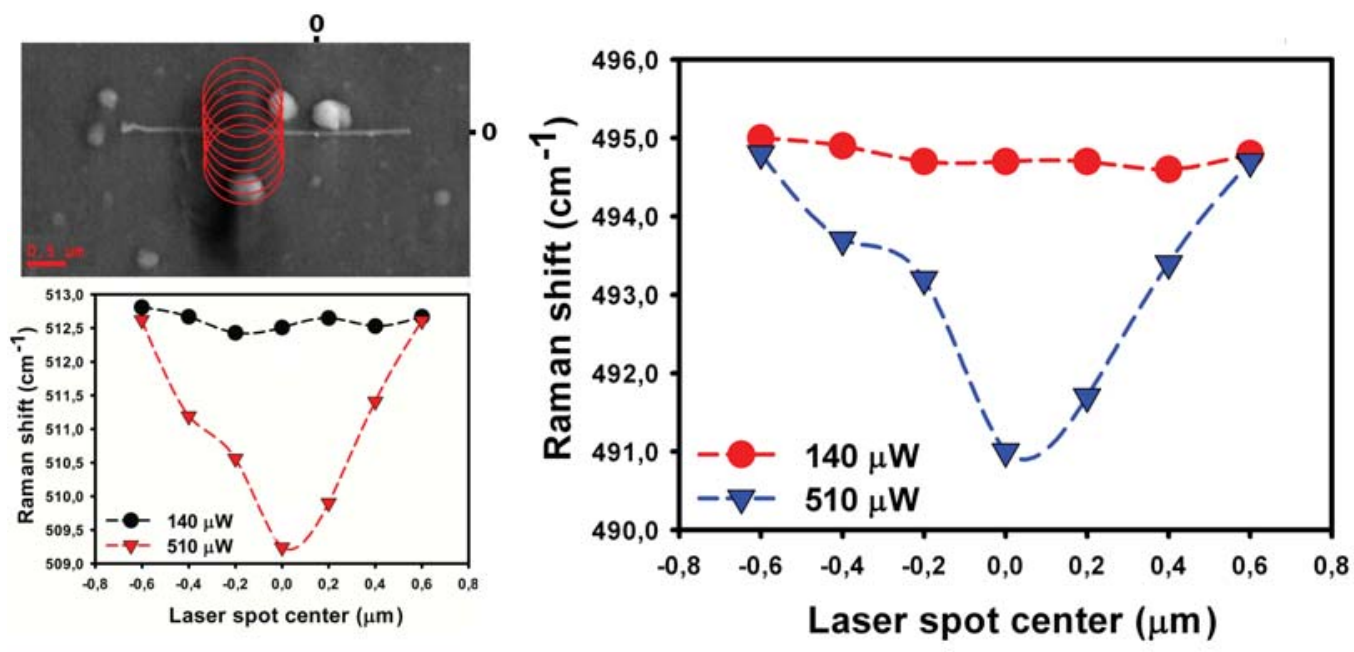

Figura 4.36: Posición del pico adyacente en un NW de silicio-germanio en función de la posición que ocupa el NW dentro del área de iluminación para dos potencias del láser Nd:YAG diferentes. Puede observarse como aparece el mismo perfil para el pico adyacente que para el pico Si-Si (Izquierda bajo la imagen SEM), por lo que ambos presentan la misma dependencia con la temperatura

en la figura 4.37-A), donde aparece representada la relación de intensidades entre el pico Si-Si y el pico suplementario a lo largo de todo el NW de silicio-germanio analizado en la figura 4.25. Este NW presentaba una composición muy homogénea $(\sim 12 \%)$ en su mayor parte, cayendo hasta un $8 \%$ en la zona de la punta del NW. Sin embargo, la máxima intensidad del pico 495 aparece en el centro del NW donde la composición es más rica en germanio, siendo casi tan intenso como el pico $\mathrm{Si}-\mathrm{Si}$; por tanto,todo apunta a que el origen del pico 495 no está relacionada directamente con los enlaces $\mathrm{Si}-\mathrm{Si}$ en el interior del NW. Este resultado ya se podía intuir al comparar las intensidades con las que aparece el pico secundario en los distintos NW analizados, puesto que pico 495 es más intenso en el NW compuesto por un $12 \%$ de germanio que en el NW compuesto por un $9 \%$, y sin embargo en NWs de la muestra del $15 \%$ o bien no aparece o bien es mucho menor que en la de $9 \%$ de germanio. En 4.36-B) se muestra la intensidad relativa en función del lugar que ocupa el NW en el área de iluminación. Dado que se está iluminando la misma zona del NW, los espectros deberían coincidir y por tanto la intensidad relativa ser constante en el corte transversal. Sin embargo, y al igual que sucedía 
para la intensidad relativa del pico Ge-Ge segregado en la figura 4.33, el espectro obtenido depende de la posición que ocupa el NW en el área de iluminación. Por tanto, ambos picos (Ge-Ge segregado y 495) se ven afectados por la posición del NW en el área de iluminación; dado que el Ge-Ge segregado se encuentra en superficie, puede pensarse que el pico adyacente en los NWs de silicio-germanio también esté relacionado con la superficie, tal y como se aventuró para los NWs de silicio. Por otro parte, el pico adyacente es más débil cuanto más rica en germanio aparece la aleación cuando se extrae la composición mediante la frecuencia del pico Ge-Ge en todos lo NWs analizados. Hasta tal punto que cuando se observa el pico Ge-Ge como germanio puro (completamente segregado) el pico adyacente desaparece por completo. Que el pico Ge-Ge aparezca completamente segregado indica que existe mucho germanio segregado en la superficie, y por tanto si el pico adyacente es un efecto de superficie de los enlaces $\mathrm{Si}$-Si, i.e. un polaritón de superficie, su existencia estaría condicionada a que no existiese una corteza de germanio. Se Volverá a incidir en este tema en el siguiente capitulo, cuando se analice la interacción electromagnética entre los NWs y el haz láser. 


\section{ESPECTROSCOPIA RAMAN EN NWS DE SILICIO Y SILICIO-GERMANIO}

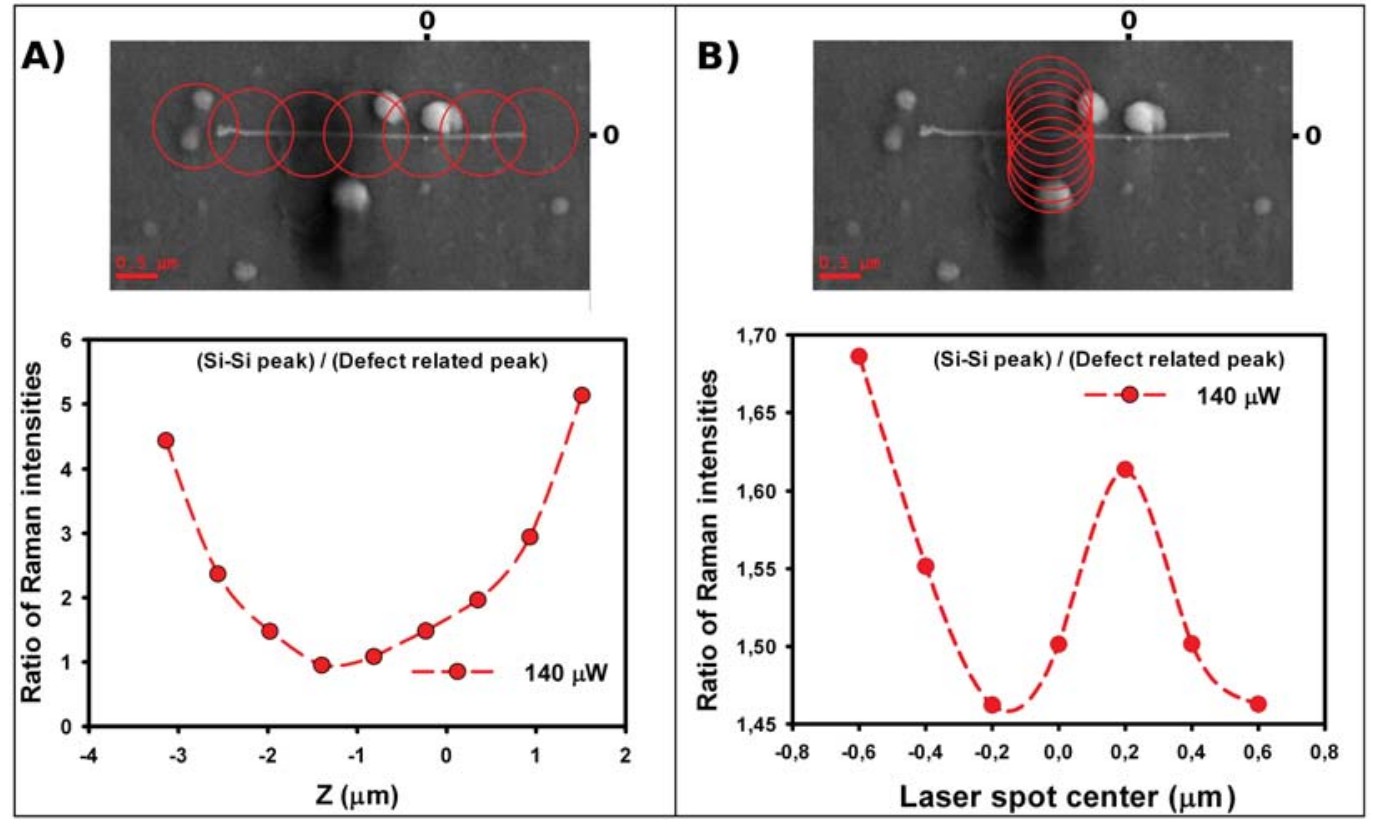

Figura 4.37: A) Intensidad relativa del pico 495 frente al pico Si-Si a lo largo de un NW de $S i_{88} G e_{12}$. B) Intensidad relativa del pico 495 frente al pico Si-Si en función de la posición que ocupa el NW en el área de iluminación habiéndose eliminado los efectos de temperatura en el experimento 


\subsection{Bibliográfia}

[1] Adu K. W, Gutierrez H. R, Kim U. J, Sumanasekera G. U, Eklund P. C. Confined Phonons in Si Nanowires, Nano Lett., 2005;5, 3.

[2] Su Z, Sha J, Pan G, Liu J, Yang D, Dickinson C, Zhou W. Temperature-Dependent Raman Scattering of Silicon Nanowire, J. Phys. Chem. B, 2006;110, 1229.

[3] Livneh T, Zhang J, Cheng G, Moskovits M. Polarized Raman scattering from single GaN nanowires, Phys. Rev. B 2006;74, 035320.

[4] Begum N, Bhatti N. S, Jabeen F, Rubini S, Martelli F. Lineshape analysis of Raman scattering from LO and SO phonons in III-V nanowires, J. Appl. Phys. 2009;106, 114317.

[5] Spirkoska D, Arbiol J, Gustafsson A, Conesa-Boj S, Glas F, Zardo I, Heigoldt M, Gass M. H, Bleloch A. L, Estrade S, Kaniber M, Rossler J, Peiro F, Morante J. R, Abstreiter G, Samuelson L, Fontcuberta i Morral1 A. Structural and optical properties of high quality zinc-blende/wurtzite GaAs nanowire heterostructures, Phys. Rev B 2009;80, 245325.

[6] Xiong Q, Chen G, Gutierrez H. R, Eklund P. C. Raman scattering studies of individual polar semiconducting nanowires: phonon splitting and antenna effects, Appl. Phys. A 2006;85, 299.

[7] Kawashima T, Imamura G, Fujii M, Hayashi S, Saitoh T, Komori K. Raman and electron microscopic studies of Si1-xGex alloy nanowires grown by chemical vapor deposition, J. Appl. Phys. 2007;102, 124307.

[8] Xiong Q, Wang J, Reese O, Lew Yan Voon L. C, Eklund P. C. Raman Scattering from Surface Phonons in Rectangular Cross-sectional w-ZnS Nanowires, Nano Lett., 2004;4, 10.

[9] Li B, Yu D, Zhang S. Raman spectral study of silicon nanowires, Phys. Rev. B 1999;59, 1645.

[10] Prades J. D, Arbiol J, Cirera A, Morante J. R, Fontcuberta i Morral A. Concerning the $506 \mathrm{~cm}-1$ band in the Raman spectrum of silicon nanowires, Appl. Phys. Lett. 2007;91, 123107.

[11] Zhou G, Liu Y, Pan S, Zhang H, Yu D, Zhang Z. Raman spectral study of silicon nanowires: High-order scattering and phonon confinement effects, Phys. Rev. B 2000;61, 16827. 


\section{ESPECTROSCOPIA RAMAN EN NWS DE SILICIO Y SILICIO-GERMANIO}

[12] Scheel H, Reich S, Ferrari A. C, Cantoro M, Colli A, Thomsen C. Raman scattering on silicon nanowires: The thermal conductivity of the environment determines the optical phonon frequency, Appl. Phys. Lett. 2006;88, 233114.

[13] Scheel H, Khachadorian S, Cantoro M, Colli A, Ferrari A. C, Thomsen C. Silicon nanowire optical Raman line shapes at cryogenic and elevated temperatures, Phys. Stat. Sol. B 2008;245, 2090.

[14] Bhattacharya S, Samui S. Phonon confinement in oxide-coated silicon nanowires. Appl. Phys. Lett. 2004;84, 1564

[15] Gupta S. R, Xiong Q, Adu C. K, Kim U. J, Eklund P. C. Laser-induced Fano resonance scattering in silicon nanowires. Nano Lett. 2003;3, 627

[16] Adu K. W, Gutiérrez H. R, Kim U. J, Eklund P. C. Inhomogeneous laser heating and phonon confinement in silicon nanowires: A micro-Raman scattering study. Phys.Rev. B 2006;73, 155333

[17] Jalilian R, Sumanasekera G. U, Chandrasekharan H, Sunkara M. K. Phonon confinement and laser heating effects in Germanium nanowires. Phys. Rev. B 2006;74, 155421

[18] Niu J, Shab J, Yang D. Temperature dependence of the first-order Raman scattering in silicon nanowires, Scripta Materialia 2006;55 183

[19] Adu K, Williams M.D, Reber M, Jayasingha R, Gutierrez H. R, Sumanasekera G. U. Probing Phonons in Nonpolar Semiconducting Nanowires with Raman Spectroscopy, Journal of Nanotechnology 2012;2012, 264198.

[20] Faraci G, Gibilisco S, Pennisi A. R, Faraci C. Quantum size effects in Raman spectra of Si nanocrystals, J. Appl. Phys. 2011;109, 074311

[21] Arora A. K, Rajalakshmi M, Ravindran T. R, Sivasubramanian V. Raman spectroscopy of optical phonon confinement in nanostructured materials, J. Raman Spectrosc. 2007;38, 604.

[22] Torres A, Martín-Martín A, Martínez O, Prieto A. C, Hortelano V, Jiménez J, Rodríguez A, Sangrador J, Rodríguez T. Micro-Raman spectroscopy of Si nanowires: Influence of diameter and temperature. Appl. Phys. Lett 2010;96, 011904 [23] Jiménez J, de Wolf I, Landesman J. P. Microprobe Characterization of Semiconductors; Optoelectronic properties of semiconductors and superlattices. Ch 2 , Vol 17, ( ed. by J. Jiménez, Taylor and Francis, New York 2002) 
[24] Hart T. R, Aggawal R.L, Lax B. Temperature Dependence of Raman Scattering in Silicon, Phys. Rev. B 1970;1, 638.

[25] Kip J. B, Meier R. J. Determination of the Local Temperature at a Sample during Raman Experiments Using Stokes and Anti-Stokes Raman Bands, Appl. Spectrocopy 1990;74, 707.

[26] Doerk S. G, Carraro C, Maboudian R. Temeperature Dependence of Raman spectra for individual silicon nanowires, Phys. Rev. B 2009;80, 073306.

[27] Chen Y, Peng B, Wang B. Raman Spectra and Temperature-Dependent Raman Scattering of Silicon Nanowires, J. Phys. Chem. C, 2007;111, 5855.

[28] Menéndez J, Cardona M. Temperature Dependece of the First-oder Raman Scattering by Phonons in Si, Ge, and $\alpha$-Sn: Anharmonic effects, Phys. Rev. B 1984;29, 2051.

[29] DulovÃ E.N, Khripunov D. M, Voigt lineshape function as a solution of the parabolic partial differential equation, Journal of Quantitative Spectroscopy \& Radiative Transfer 2007;107, 421.

[30] Cao L, Fan P, Brongersma M. L, Optical Coupling of Deep-Subwavelength Semiconductor Nanowires, Nano Lett. 2011;11, 1463.

[31] Omar M.S, Taha H.T. Lattice dislocation in Si nanowires. Physica B 2009;404, 5203. [32] Raman C.V, The vibration spectra of crystals. Part I. Basic Theory. Proceedings of the Indian Academy of Sciences - Section A, 1947;26, 339.

[33] Narayanaswamy P. K, Influence of temperature on the Raman spectra of crystals. Proceedings of the Indian Academy of Sciences - Section A, 1948;28, 40.

[34] Lopez F. J, Hemesath R. R, Lauhon L. J. Ordered Stacking Fault Arrays in Silicon Nanowires, Nano Lett. 2009;9, 2774.

[35] Nikolenko A, Strelchuk V, Klimovskaya A, Lytvyn P, Valakh M, Pedchenko Y, Voroschenko A, Hourlier A. Scanning confocal Raman spectroscopy of silicon phase distribution in individual Si nanowires, Phys. Status Solidi C 2011;8, 1012.

[36] Prades D, Arbiol J, Cirera A, Morante J. R, Fontcuberta i Morra A. Concerning the $506 \mathrm{~cm}^{-1}$ band in the Raman spectrum of silicon nanowires, Appl. Phys. Lett. 2007;91, 123107.

[37] Peng Z, Hu H, Bakti Utama M. I, Wong L. M, Ghosh K, Chen R, Wang S, Shen Z, Xiong Q. Heteroepitaxial Decoration of Ag Nanoparticles on Si Nanowires: A Case Study on Raman Scattering and Mapping, Nano Lett. 2010;10, 3940. 


\section{ESPECTROSCOPIA RAMAN EN NWS DE SILICIO Y SILICIO-GERMANIO}

[38] GaÏsler S. V, Semenova O. I, Sharafutdinov R. G, Kolesov B. A. Analysis of Raman Spectra of Amorphous-Nanocrystalline Silicon Films, Physics of the Solid State, 2004;46, 1528.

[39] Barth S, Boland J. J, Holmes J. D. Defect Transfer from Nanoparticles to Nanowires, Nano Lett. 2011;11, 1550.

[40] Bandet J, Despax B, Caumont M. Vibrational and electronic properties of stabilized wurtzite-like silicon, J. Phys. D: Appl. Phys. 2002;35, 234.

[41] Lee N, Hartschuh R. D, Mehtani D, Kisliuk A, Maguire J. F, Green M, Foster M. D, Sokolov A. P. High contrast scanning nano-Raman spectroscopy of silicon, J. Raman Spectrosc. 2007;38, 789.

[42] Son J, Nam H, Cho N. Nanostructural, Chemical, and Mechanical Features of nc-Si:H Films Prepared by PECVD, International Journal of Photoenergy 2012;2012, 643895.

[43] Viera G, Huet S, Boufendi L. Crystal size and temperature measurements in nanostructured silicon using Raman spectroscopy, J. Appl. Phys.,2001;90, 4174.

[44] Mahan G. D, Gupta R, Xiong Q, Adu C. K, Eklund P. C. Optical phonons in polar semiconductor nanowires, Phys. Rev. B, 2003;68, 073402.

[45] Begum N,1, Piccin M,1, Jabeen F, Bais G, Rubini S, Martelli F, Bhatti A. S. Structural characterization of GaAs and InAs nanowires by means of Raman spectroscopy, J. Appl. Phys. 2008;104, 104311.

[46] Hayashi F. Optical Study of Electromagnetic Surface Modes in Microcrystals, J. J. Appl. Phys. 1984;23, 665.

[47] Ushioda S, Aziza A, Valdez J. B, Mattei G. Effects of Surface Roughness on Surface Polaritons, Phys. Rev. B, 1979;19, 4012.

[48] Fleischer K, Chandola S, Esser N, Richter W, McGilp J. F. Surface phonons of the Si (111), Phys. Reb B 2007;76, 205406

[49] Cardona M, Guntherodt G. Ligth Scattering in Solids, Topics in applied physics, Springer Verlag, Heilderberg, Vols 8, 50, 51, 54, 66, 68.

[50] Cao L, Laim, Valenzuela P. D, Nabet B, Spanier J. E. On the Raman scattering from semiconducting nanowires, J. Raman Spectrosc. 2007;38, 697.

[51] Cao L, Nabet B, Spanier J. E. Enhanced Raman Scattering from Individual Semiconductor Nanocones and Nanowires, Phys. Rev. Lett. 2006;96, 157402.

[52] Haynes C. L, McFarland A. D, Van Duyne R. P. Surface-Enhanced Raman 
Spectroscopy, Anal. Chem., 2005; 77, 338.

[53] Feldman D.W, Ashkin, M, Parker J.H. Raman Scattering by Local Modes in Germanium-Rich Silicon-Germanium Alloys, Phys. Rev. Letters, 1966;17, 1209.

[54] Brya W.J. Raman scattering in Ge-Si alloys, Solid State Commun., 1973;12, 253.

[55] Alonso M.I, Winer K. Raman Spectra of $c-S i_{1-X} G e_{X}$ Alloys, Phys. Rev. B, 1989;39, 10056.

[56] de Gironcoli S, Baroni S. Effects of disorder on the vibrational properties of SiGe alloys: Failure of mean-field approximations , Phys. Rev. Letters, 1992;69, 1959.

[57] Ren S, Cheng W, Yu P. Y. Microscopic Investigation of Phonon Modes in SiGe Alloy Nanocristals, Phys. Rev. B, 2004;69, 235327.

[58] Islam M. R, Yamada M. Raman scattering characterization of Ge-composition in bulk Si1-xGex with compositional variation, J Mater Sci: Mater Electron, 2008;19, S294.

[59] Tsang J. C, Mooney P. M, Dacol F, Chu J.O. Measurements of alloy composition and strain in thin GeXSi1-X layers, J. Appl. Phys., 1994;75, 8098.

[60] Pezzoli F, Martinelli L, Grilli E, Guzzi M, Sanguinetti S, Bollani M, Chrastina H. D, Isella G, von Kanel H, Wintersberger E, Stangl J, Bauer G. Raman spectroscopy of Si 1-xGex epilayers, Materials Science and Engineering B, 2005;124-125, 127.

[61] Perova T. S, Wasyluk J, Lyutovich K, Kasper E, Oehme M, Rode K, Waldron A. Composition and strain in thin Si $1-x G e x$ virtual substrates measured by microRaman spectroscopy and $x$-ray diffraction, J. Appl. Phys. 2011;109, 033502.

[62] Nakashima S, Mitani T, Ninomiya M, Matsumoto K. Raman investigation of strain in Si/ SiGe heterostructures: Precise determination of the strain-shift coefficient of Si bands, J. Appl. Phys. 2006;99, 053512.

[63] Hull J, Bean C, Germanium Silicon: Physics and Materials, SEMICONDUCTORS AND SEMIMETALS Vol 56, Edit by Hull J, Bean C, 1999, ACADEMIC PRESS, San Diego. [64] Holtz M, Duncan W. M, Zollner S, Liu R. Visible and Ultravioles Raman Scattering Studies os Sil-XGeX Alloys, J. Appl. Phys., 2000;88, 2523.

[65] Monasterio M, Rodríguez A, Rodríguez T, Ballesteros C. SiGe Nanowires 


\section{ESPECTROSCOPIA RAMAN EN NWS DE SILICIO Y}

SILICIO-GERMANIO

Grown by LPCVD using Ga-Au Catalyst. Mater. Res. Soc. Symp. Proc. 2012, DOI:10.1557/opl.2012.34.

[66] Wu J. Polarized Ligth Scattering from Individual Semiconductor Nanowires, Tesis de la Universidad de Pennsylvania State, 2008.

[67] Kawashima T, Imamura G, Fuji M, Hayashi S, Saitoh T, Komori K. Raman and Electron Microscopic Studies of Si1-XGeX Alloy Nanowires Grown by Chemical Vapor Deposition, J. Appl. Phys. 2007;102 124307.

[68] Burke H. H, Hereman I. P. Temperature dependence of Raman scattering in Ge1-xSix alloys, Phys. Rev. B 1993;48, 15016.

[69] Godbey D. J, Ancona M. G. Ge Segregation During the Growth of SiGe Buried Layer by Molecular Beam Epitaxy, J. Vac. Sci. Technol. B, 1993;11, 1120.

[70] Min B. G, Pae Y. H, Jun K. S, Ko D. H, Kim H, Cho M. H, Lee T. W. Formation of a Ge-rich layer during the oxidation of strained Sil-xGex, J. Appl. Phys. 2006;100, 016102.

[71] Lew K, Pan L, Dickey E. C, Redwing J. M. Effect of Growth Conditions on the Composition and Structure of Sil-XGeX Nanowires Grown by Vapor-Liquid-Solid Growth, J. Mater. Res. 2006;21, 2876. 
Accurate and minute measurement seems to the non-scientific imagination, a less lofty and dignified work than looking for something new. But nearly all the grandest discoveries of science have been but the rewards of accurate measurement and patient long-continued labour in the minute sifting of numerical results.

Baron William Thomson Kelvin

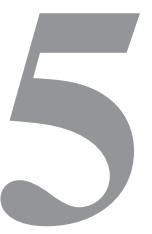

\section{Estudio del Campo electromagnético en el interior de un NW semiconductor excitado con un haz láser polarizado}

\subsection{Introducción}

En los capítulos anteriores se ha descrito el problema de la interacción láser-NW y su influencia en el espectro Raman desde un punto de vista térmico. La descripción teórica de esta interacción ha resultado fundamental a la hora de interpretar correctamente los espectros Raman obtenidos, así como de establecer unas condiciones de experimentación adecuadas para la caracterización Raman en NWs de silicio y silicio-germanio. Sin embargo, cuando se ha realizado la caracterización experimental de los NWs mediante espectroscopia Raman, se han observado otros efectos anómalos que nada tienen que ver con la interacción térmica láser-NW, y que deben ser atribuidos a otros efectos de tipo puramente electromagnético. 


\section{ESTUDIO DEL CAMPO ELECTROMAGNÉTICO EN EL INTERIOR DE UN NW SEMICONDUCTOR EXCITADO CON UN HAZ LÁSER POLARIZADO}

De forma muy resumida, cuando se iluminan los NWs con un haz láser, el campo electromagnético (láser) induce un polarización en los enlaces moleculares del NW. Esta polarización puede separarse en dos componentes: un término estático que oscila con el campo electromagnético incidente, responsable del scattering Rayleigh, y un termino de polarización inducido por los desplazamientos de la red [1] (ver Capítulo 1). Este segundo término engloba el scattering Raman Stokes y anti-Stokes, y la intensidad con la que se irradian los fotones procedentes de este scattering Raman se determina a partir de la expresión [2]:

$$
I_{R}=\left(\frac{\omega^{2} \mu_{0}\left|E_{0}\right|}{4 \pi R}\right)^{2}<\left|e_{\alpha}^{i n c} \alpha e_{\alpha}^{s c a t}\right|^{2}>
$$

Donde $\omega$ es la frecuencia del láser incidente , $\mu_{0}$ la permeabilidad magnética del NW, $|E|$ el modulo del campo eléctrico en el interior del NW, $R$ la distancia entre el detector y la muestra y finalmente $<\left|e_{\alpha}^{i n c} \alpha e_{\alpha}^{s c a t}\right|>$ es el tensor Raman característico del material. Este tensor Raman está caracterizado a su vez por el tensor de polarizabilidad, $\alpha$, y los vectores de polarización incidente y reflejada, $e_{\alpha}^{i n c}, e_{\alpha}^{s c a t}$ respectivamente.

Observando la expresión 5.1 que caracteriza la intensidad Raman, resulta evidente que la señal procedente de los NWs va a estar muy condicionada por cómo es el campo eléctrico en el interior de los NWs cuando estos son iluminados por un haz láser, y por tanto, para entender en profundidad la señal Raman proveniente de los NWs, es necesario realizar un análisis detallado de la interacción electromagnética láser-NW.

Cuando se analizó la interacción láser-NW en el capitulo 3, una de las cosas que se tuvo que tener en cuenta es que el diámetro de los NWs es menor que la longitud de onda de la radiación incidente. Este aspecto va a ser de nuevo determinante a la hora de analizar como es el campo eléctrico en el interior de los NWs [3]. La distribución del campo eléctrico en el interior de NWs semiconductores de diferentes materiales bajo un campo electromagnético ha sido recientemente estudiada por algunos autores $[3-7]$, siendo un campo de investigación muy reciente y poco explorado por el momento. Xiong et al., mediante una simulación realizada mediante DDA (Discrete-Dipole Aprox. ) mostraron en [3] como el campo eléctrico en el interior de NWs de GaP, tanto en la intensidad, como en su distribución en el 
interior de los mismos, dependía fuertemente del diámetro del NW. Con el mismo método (DDA), Chen et al. mostraron como también la polarización del haz incidente era determinante a la hora de evaluar la distribución del campo eléctrico en el interior de los NWs de GaP [4]. Por último, Lopez et al., utilizando un método FDTD (Finite Diference in Time Domain), han estudiado muy recientemente este mismo efecto en NWs de silicio [7], mostrando como el campo eléctrico en el interior de estos NWs se distribuye de forma muy inhomogénea y localizada, dependiendo fuertemente del diámetro del NW. Que el campo eléctrico en los NWs de silicio esté muy localizado en el interior va a tener una gran relevancia a la hora de entender los espectros Raman procedentes de estos NWs, ya que implica que la señal obtenida no proviene de todo el volumen iluminado del NW por igual, sino que procede precisamente de zonas localizados del NW [7].

\subsection{Modelado de la interacción electromagnética para el conjunto láser-NW-substrato}

El modelado de la interacción electromagnética entre el láser, el NW y el substrato sobre el que reposa, requiere resolver completamente las ecuaciones de Maxwell que describen todo el proceso, resultando en un problema muy complejo que sólo pude ser abordado mediante cálculo numérico. Este tipo de problemas que envuelven la propagación de una onda electromagnética y su interacción con diversos obstáculos en su camino de propagación, típicamente se resuelven utilizando métodos FDTD [7, 8], o más raramente métodos DDA [3, 4, 9] con muy buenos resultados. Sin embargo, y dado que no tenemos las herramientas necesarias para desarrollar un modelo bajo estos formalismos, en este trabajo se ha recurrido a un formalismo diferente, y no utilizado hasta el momento para estudiar la interacción en NWs, basado en un método de elementos finitos (FEM), para atacar la resolución del problema que representa la interacción láser-NW-substrato. Para ello, se ha recurrido al modulo $R F$ de COMSOL Multiphysics, el cual permite modelar problemas de propagación de ondas electromagnéticas y el análisis de la interacción de estas ondas cuando se encuentran con un objeto (dieléctrico,metal, etc..) 


\section{ESTUDIO DEL CAMPO ELECTROMAGNÉTICO EN EL INTERIOR DE UN NW SEMICONDUCTOR EXCITADO CON UN HAZ LÁSER POLARIZADO}

en su camino de propagación. El análisis de la interacción láser-NW-substrato se lleva a cabo en un modelo simplificado a dos dimensiones, asumiendo para ello que dadas las enormes diferencias entre las dimensiones radiales y longitudinales, y asumiendo que se ilumina el NW en su zona central, la solución 2D no se aleja demasiado de la realidad. Esta aproximación de NWs infinitos se ha utilizado en los cálculos realizados en $[3,5,6,7,14]$ por diversos autores para resolver un problema equivalente. Los dos láseres utilizados en la espectroscopia Raman llevada a cabo con nuestro equipo, el He-Ne y el Nd:YAG doblado, se caracterizan por emitir en el modo TEM 00, es decir en el modo fundamental transversal electromagnético caracterizado por una distribución gaussiana de la potencia en el foco [10]. Bajo la aproximación paraxial, puede asumirse que en este modo TEM 00, las componentes del campo electromagnético en la dirección de propagación del haz láser son nulas $[10,11]$. Además, la luz que sale por el objetivo de nuestro equipo Raman está linealmente polarizada, por lo que el haz láser gaussiano puede expresarse bajo la aproximación paraxial de orden más bajo como [10 - 12]:

$$
\begin{gathered}
E_{z}(x, y, t)=E_{0} \frac{w_{0}}{w(x)} e^{\left(-\frac{y^{2}}{w(x)^{2}}\right)} e^{\left(i k \frac{y^{2}}{2 R(x)}\right)} e^{-i \zeta} e^{i(k x-\omega t)} \\
H_{y}(x, y, t)=\left(\frac{E_{0}}{c \mu_{0}}\right) \frac{w_{0}}{w(x)} e^{\left(-\frac{y^{2}}{w(x)^{2}}\right)} e^{\left(i k \frac{y^{2}}{2 R(x)}\right)} e^{-i \zeta} e^{i(k x-\omega t)}
\end{gathered}
$$

Donde se asume que la dirección de propagación es a lo largo del eje $X$, tal y como se muestra en la figura 5.1. El término $E_{0}$ es la amplitud del campo eléctrico, que está relacionada con la potencia del láser. La anchura del spot gaussiano del láser se tiene en cuenta en el término $w(x)$ y se obtiene como:

$$
w(x)=w_{0} * \sqrt{1+\left(\frac{\lambda x}{\pi w_{0}^{2}}\right)^{2}}
$$

Donde se ha fijado $w_{0}$ a $1 \mu$ de diámetro para ambos láseres por simplificar el problema sin perdida de generalidad por ello. Por otro lado, $R(x)$ da cuenta del factor de curvatura del haz láser [10], y se obtiene como:

$$
R(x)=x\left(1+\left(\frac{\pi w_{0}^{2}}{\lambda x}\right)^{2}\right)
$$




\subsection{Modelado de la interacción electromagnética para el conjunto láser-NW-substrato}

Finalmente $\eta(x)$ es la denominada fase Guoy característica de los frentes de onda esféricos y gaussianos [13]:

$$
\eta(x)=\arctan \left(\frac{\pi w_{0}^{2}}{\lambda x}\right)
$$

Las ecuaciones 5.2 que describen la propagación del haz láser gaussiano linealmente polarizado se han introducido en el modelo generado con el modulo $R F$ de $C O M S O L$, cuyas características pueden verse en la figura 5.1. En 5.1-A) pueden verse el dominio de simulación establecido para resolver el problema. El haz gaussiano se propaga en el dominio de simulación a través del aire, hasta focalizarse en el NW. Este NW está situado sobre un volumen que dependiendo de las condiciones establecidas para su indice de refracción puede ser cualquier material (Aluminio, oro, germanio, etc...) o bien puede establecerse que tenga idénticas características al volumen por donde se propaga el láser en aire, de modo que se simule la interacción láser-NW en un NW completamente rodeado por aire. Para garantizar que se realiza un modelo equivalente a la situación real que se tiene en la espectroscopia Raman, se ha construido un dominio suficientemente grande para que las fronteras interfieran lo menos posible en la solución, y además se ha rodeado todo el dominio de simulación de PML's (Perfect Matched Layers) cartesianas, las cuales se encargan de absorber perfectamente toda la radiación que les llega, de modo que se eliminan reflexiones secundarias que inducirían un calculo erróneo del campo electromagnético en el dominio de simulación $[15,16]$. En 5.1-B) se puede apreciar como está modelado el NW dentro del dominio de simulación para que sea compatible tanto para NWs de silicio como para NWs de silicio-germanio, para los cuales las medidas experimentales indican que están compuestos por un núcleo de silicio-germanio y por una pequeña coraza de germanio segregado. Finalmente, sobre este dominio de simulación se han resulto las ecuaciones del campo electromagnético dadas por:

$$
\begin{array}{r}
\nabla \times\left(\nabla \times E_{i}\right)-n^{2} k_{0}^{2} E_{i}=0 \\
\nabla \times\left(n^{-2} \nabla \times H_{j}\right)-k_{0}^{2} H_{j}=0
\end{array}
$$




\section{ESTUDIO DEL CAMPO ELECTROMAGNÉTICO EN EL INTERIOR DE UN NW SEMICONDUCTOR EXCITADO CON UN HAZ LÁSER POLARIZADO}

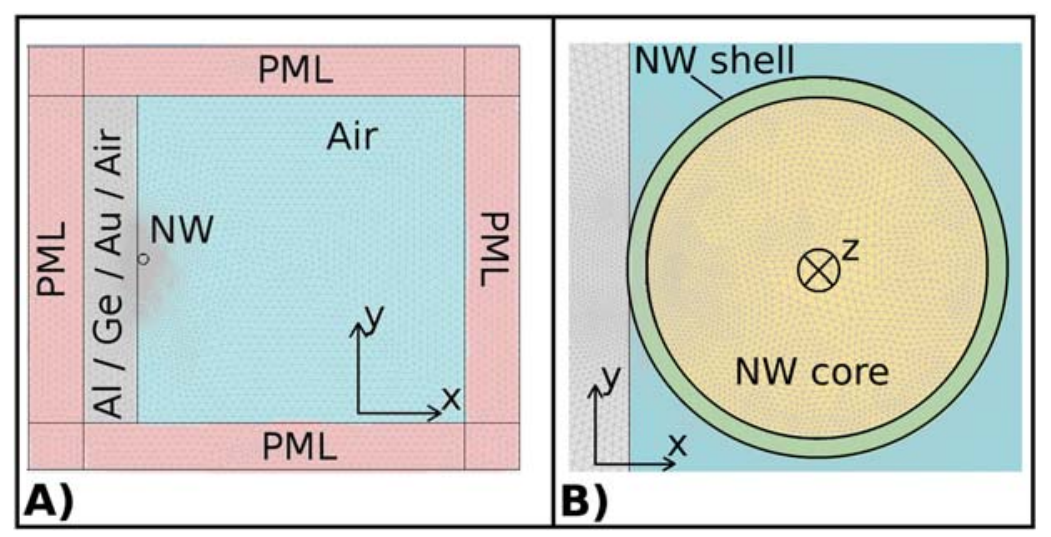

Figura 5.1: A) Dominio de simulación implementado en $C O M S O L$ para la resolución del problema electromagnético resultante de iluminar NWs semiconductores con un haz láser. El NW se encuentra depositado sobre un volumen que hace las veces de substrato, cuando se simula un NW rodeado completamente por aire este volumen desaparece. B) Ampliación del NW mostrado en A), puede observarse como el modelo permite resolver el problema tanto para NWs de silicio (coraza y núcleo idénticos) como para NWs de germanio con germanio segregado (Corteza de germanio y núcleo de silicio-germanio)

Donde $n$ es el indice de refracción complejo y las componentes de $E_{i}$ y $H_{j}$ son determinadas a través de las ecuaciones 5.2 teniendo en cuenta el ángulo que forma el NW con el eje de polarización del haz láser, de tal modo que cuando el eje del NW está alineado con el eje de polarización del Haz láser (i.e. como se muestra en 5.1-A)) $H_{j}=0$, mientras que cuando se gira $90^{\circ}$ el NW (i.e. el eje del NW es paralelo a la dirección $y$ de la figura 5.1-A)) se tiene que $E_{i}=0$. Todos los ángulos intermedios entre estas posiciones se simulan a través de las proyecciones de $E$ y $H$ sobre la sección del NW.

Para validar el modelo así construido, se ha resuelto el problema para NWs de silicio depositados sobre un substrato de oro, iluminados por un haz láser de 532 $\mathrm{nm}$ de longitud de onda y $1 \mu \mathrm{m}$ de diámetro de spot láser linealmente polarizado y con los NWs alineados con el eje de polarización (i.e. $H(z)=0$ ) y para todos los diámetros que fueron resueltos por FDTD en [7] bajo estas mismas condiciones. En 5.2-A) se muestra como la solución para $|E|^{2}$ en el interior de los NWs alcanzada por ambos modelos en idénticas condiciones de simulación ofrecen idéntico resultado, confirmando la validez del modelo FEM desarrollado. Resulta interesan- 

substrato en el valor de $|E|^{2}$ en el interior de los NWs.

te observar como en función del diámetro, la distribución de $|E|^{2}$ en el interior de los NWs cambia notablemente. Además, resulta especialmente interesante que para NWs con diámetros comprendidos entre $\sim 50 \mathrm{~nm} y \sim 100 \mathrm{~nm},|E|^{2}$ está concentrado en las zonas más cercanas a la superficie, mientras que el núcleo del NW tiene un valor de $|E|^{2}$ varios ordenes de magnitud menor. Esto implica que la señal Raman, que recordemos es proporcional a $|E|^{2}$ (ver ecuación 5.1), procede casi exclusivamente de las zonas próximas a la superficie del NW. Cuando tenemos diámetros menores a $40 \mathrm{~nm}$ y superiores a 150 , la distribución de $|E|^{2}$ es más intensa en el interior del NW, por lo que la señal Raman procede mayoritariamente de las partes interiores del NW. Por otro lado, la eficiencia de Absorción y el valor de $|E|^{2}$ son directamente proporcionales [7], por lo que ambos deben seguir el mismo comportamiento en función del diámetro. En 5.2-B) se compara la eficiencia de absorción calculada a través de la ecuación 3.4 procedente de formalismo de Mie [17], con la solución obtenida para el valor de la eficiencia de absorción calculado a través del valor integrado de $|E|^{2}$ cuando el NW está rodeado por aire. Puede observarse como ambas formas ofrecen una solución muy similar en todo el rango de diámetros simulado, sólo apareciendo una pequeña discrepancia para diámetros menores a 30 nm. Cabe destacar que la solución mediante el formalismo de Mie es una aproximación más grosera que la resolución de las ecuaciones completas de la interacción que se lleva a cabo en el modelo FEM [17].

\subsection{Influencia de las dimensiones del NW, longitud de onda y efecto del substrato en el valor de $|E|^{2}$ en el interior de los NWs.}

Una vez que nuestro modelo se ha validado comprobando sus soluciones con las arrojadas por otros métodos sobre los mismos problemas, podemos utilizarlo para analizar que efecto tienen los diversos factores que intervienen en la interacción electromagnética láser-NW-substrato.

Los valores del indice de refracción de cada material utilizados para resolver la ecuación 5.6 en cada caso se muestran en la tabla 5.1. En la figura 5.3-A) se muestra como la distribución de $|E|^{2}$ en el interior de un NW de silicio es prácticamente 


\section{ESTUDIO DEL CAMPO ELECTROMAGNÉTICO EN EL INTERIOR DE UN NW SEMICONDUCTOR EXCITADO CON UN HAZ LÁSER POLARIZADO}

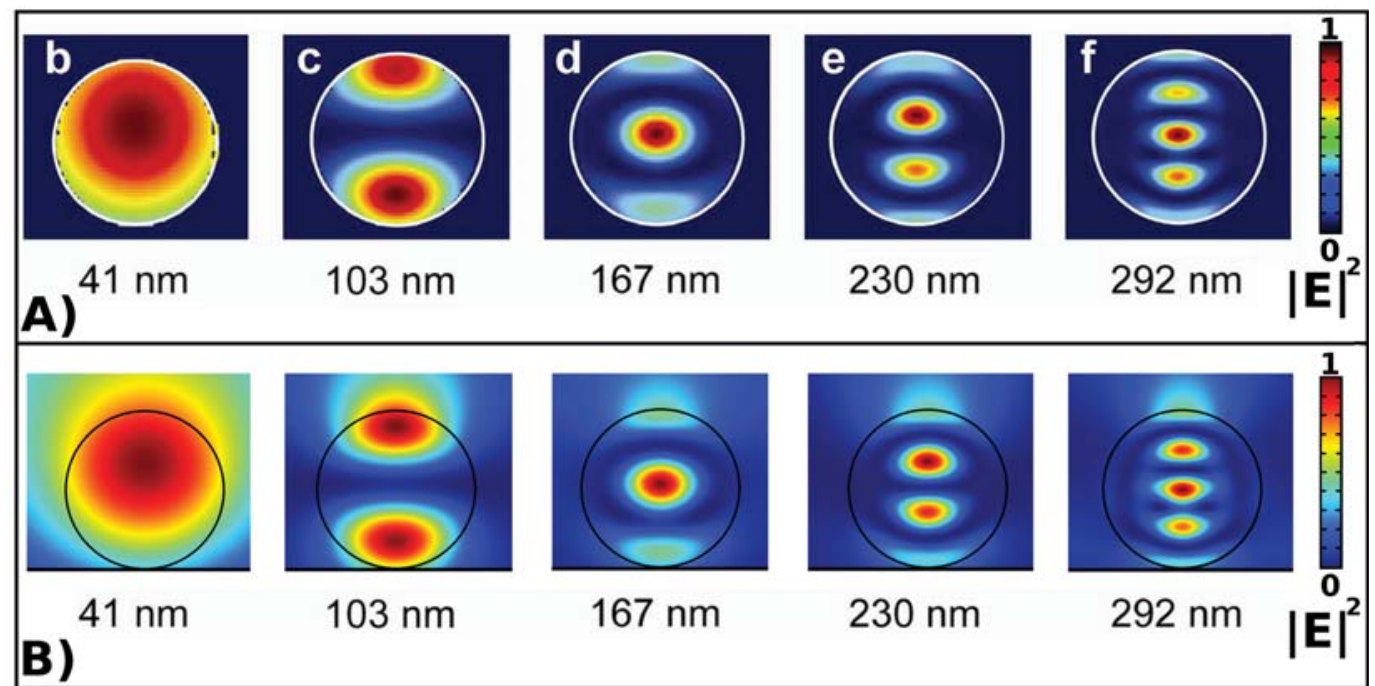

C)

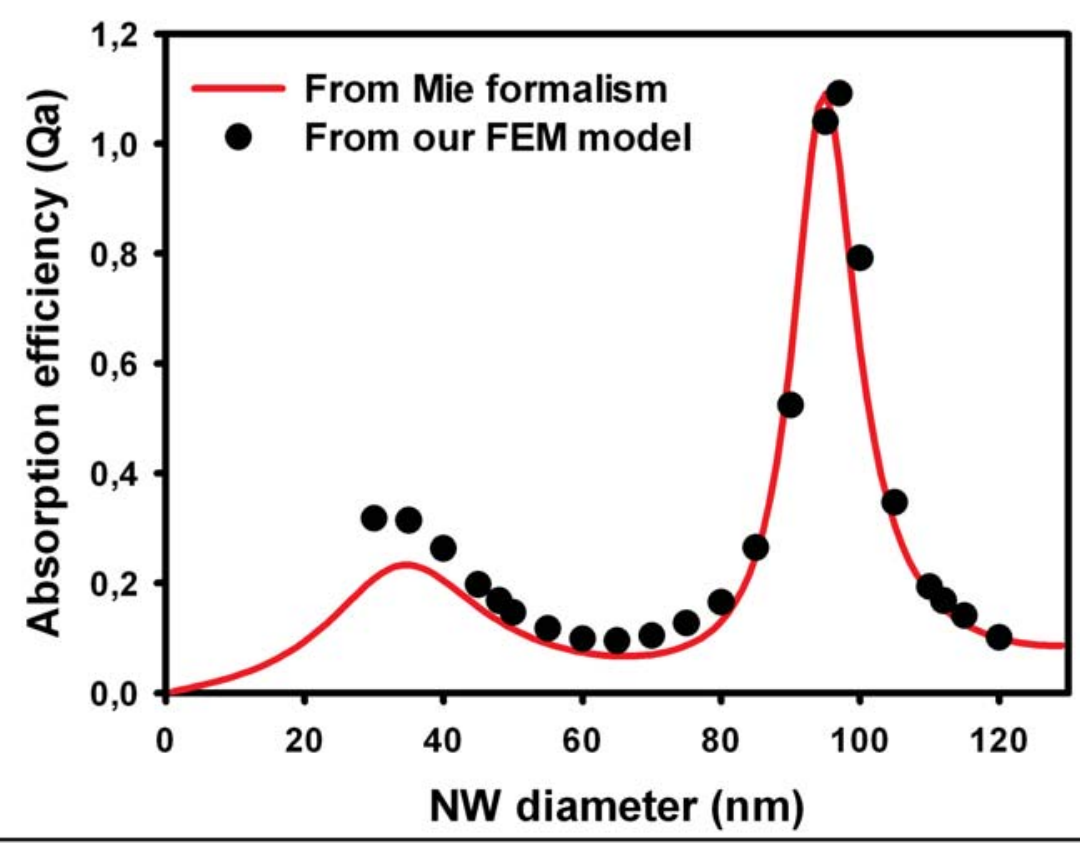

Figura 5.2: A) $|E|^{2}$ normalizado calculado por FDTD en [7] en el interior de NWs de silicio iluminados con un haz láser de $532 \mathrm{~nm}$ y situados sobre un substrato de oro. B) Solución para las mismas condiciones obtenida con el modelo FEM aquí desarrollado. Puede observarse la perfecta correspondencia entre ambas soluciones. C) Eficiencia de absorción calculada a través del formalismo de Mie y valor normalizado de $|E|^{2}$ obtenido mediante el modelo FEM. 
5.3 Influencia de las dimensiones del NW, longitud de onda y efecto del substrato en el valor de $|E|^{2}$ en el interior de los NWs.

\begin{tabular}{|l|c|c|c|c|c|c|}
\hline & Aire & Germanio & Aluminio & Oro & Silicio & $S i_{85} G e_{15}$ \\
\hline $532.8 \mathrm{~nm}$ & 1.00027316 & $4.92436+2.3734 \mathrm{i}$ & $0.93877+6.4195 \mathrm{i}$ & $0.467+2.4075 \mathrm{i}$ & $4.1334+0.033258 \mathrm{i}$ & $4.26993+0.040503 \mathrm{i}$ \\
\hline $632.8 \mathrm{~nm}$ & 1.00027654 & $5.47932+0.7997 \mathrm{i}$ & $1.44308+7.5285 \mathrm{i}$ & $0.1984+3.0875 \mathrm{i}$ & $3.8841+0.01977 \mathrm{i}$ & $3.97514+0.015625 \mathrm{i}$ \\
\hline
\end{tabular}

\section{Cuadro 5.1: .}

]Valores de los indices de refracción utilizados en las simulaciones para cada material procedentes de la referencia [18].

la misma con independencia del substrato sobre el que se apoya. Sin embargo, el efecto del substrato es muy grande en el valor que alcanza $|E|^{2}$ en el interior de los NWs. En la figura 5.3-B), se muestra el valor máximo de $|E|^{2}$ en el interior de los NWs de silicio con diámetros comprendidos entre 30 y 120 nm en función del substrato donde se apoyan en cada caso. Puede observarse como aparecen resonancias para algunos diámetros de NW en las cuales el valor de $|E|^{2}$ llega ser varios ordenes de magnitud superior al que se alcanza cuando el NW está rodeado por aire. Dependiendo del substrato, la resonancia en $|E|^{2}$ se produce a diferentes diámetros. En 5.3-B) se representa el incremento del valor de $|E|^{2}$, integrado a la sección del NW, para los NWs depositados en un substrato frente al mismo valor de $|E|^{2}$ calculado en los NWs rodeados de aire. Dependiendo del substrato y del diámetro del NW pueden obtenerse valores de $|E|^{2}$ hasta 3 ordenes de magnitud superiores con respecto a los valores correspondientes a los mismos NWs rodeados por aire. Por lo tanto, la señal Raman de los NWs cuyo diámetro se encuentra en resonancia con el substrato puede ser hasta mil veces mayor que la obtenida de un NW rodeado por aire (e.g. NWs similares al mostrado en la figura 4.7). En la figura 5.4 se muestra lo mismo que aparece en la 5.3 pero cambiando la longitud de onda del láser a $632.8 \mathrm{~nm}$. Puede observarse como de nuevo aparece el mismo comportamiento ya mostrado para el láser de $532 \mathrm{~nm}$, pero ahora los diámetros para los que aparecen las resonancias en $|E|^{2}$ se han desplazado a diámetros más grandes en todos los casos analizados. Este efecto introducido por el substrato puede explicar porqué veíamos una intensidad tan grande en los NWs analizados en el capitulo anterior.

Una vez entendido el papel del substrato en la señal Raman, se abre la puerta a sintonizar para cada NW de un determinado material un substrato que ofrezca un máximo de $|E|^{2}$ y por tanto de intensidad Raman. Esto puede ser de gran importancia para NWs construidos con materiales que tienen una señal Raman tan baja que 


\section{ESTUDIO DEL CAMPO ELECTROMAGNÉTICO EN EL INTERIOR DE UN NW SEMICONDUCTOR EXCITADO CON UN HAZ LÁSER POLARIZADO}

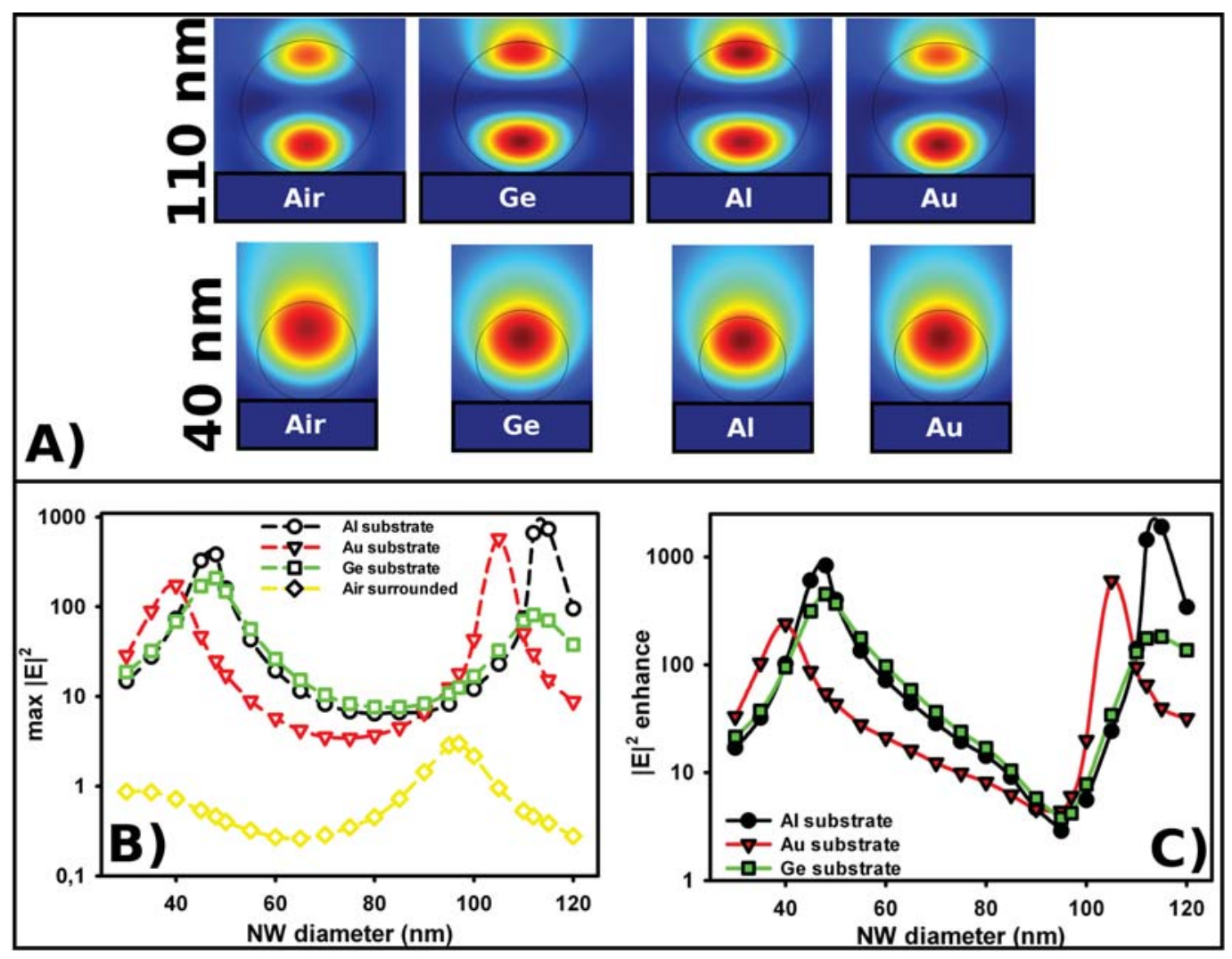

Figura 5.3: A) Distribución de $|E|^{2}$ en función del substrato para dos NWs de silicio de 110 y $40 \mathrm{~nm}$ de diámetro que son iluminados por un haz láser de $532 \mathrm{~nm}$. B) valor máximo de $|E|^{2}$ en el interior de los NWs de silicio en función de su diámetro y del substrato sobre el que se apoyan. Se ha considerado $E_{0}=1$ en la ecuación 5.2 por simplicidad y sin perdida de generalidad del resultado. C) Aumento del valor de $|E|^{2}$ integrado en la sección del NW de los NWs sobre un substrato respecto al valor de $|E|^{2}$ procedente del NW rodeado por aire. Nótese como pueden aparecer incrementos en $|E|^{2}$ de hasta tres ordenes de magnitud para algunos diámetros y substratos, lo que implica un aumento en la señal Raman de tres ordenes de magnitud. 
5.3 Influencia de las dimensiones del NW, longitud de onda y efecto del substrato en el valor de $|E|^{2}$ en el interior de los NWs.

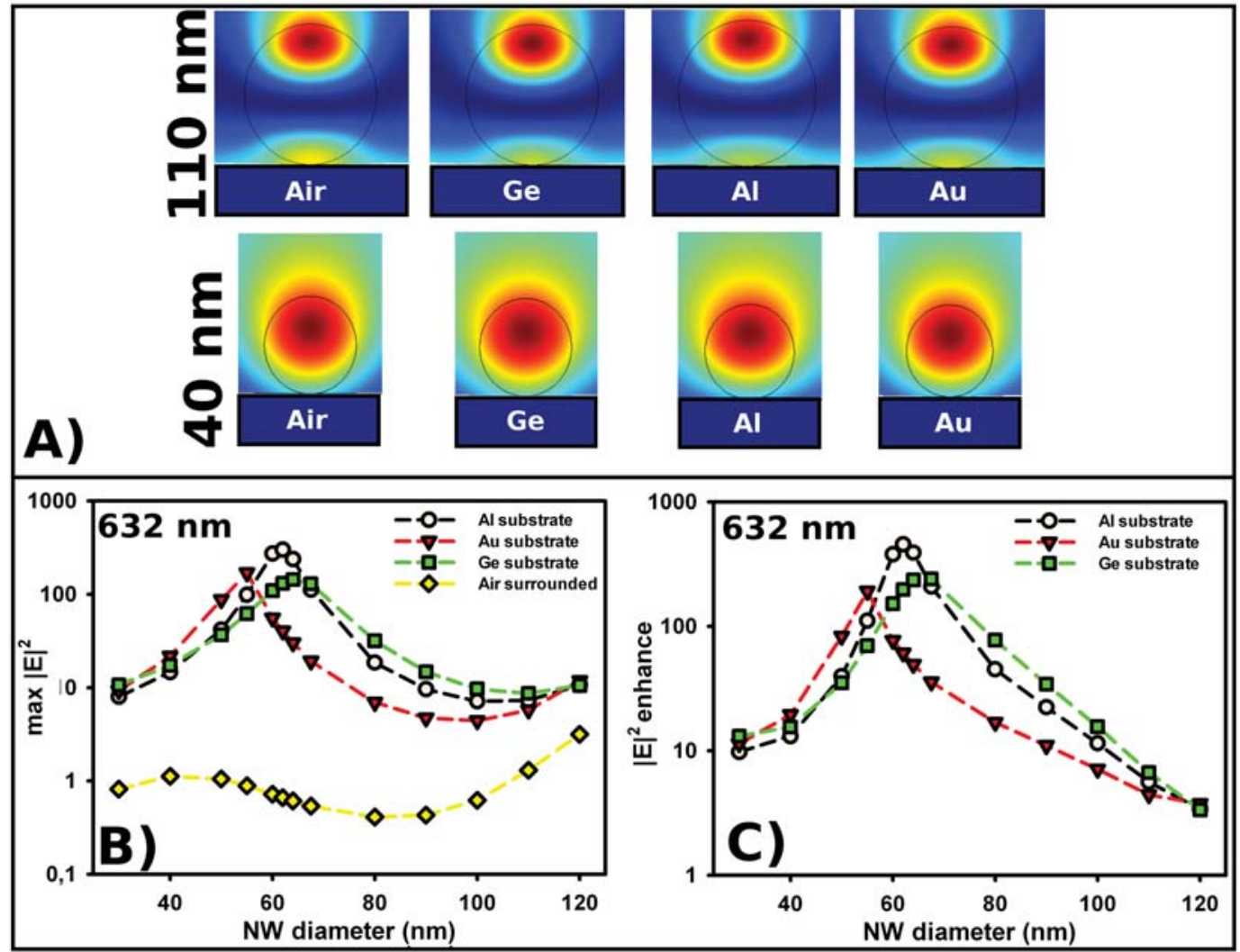

Figura 5.4: Misma figura que la mostrada en 5.3-A, B y C pero cambiando la longitud de onda de la radiación incidente a $632.8 \mathrm{~nm}$. Obsérvese que las resonancias en $|E|^{2}$ siguen apareciendo, pero se desplazan en todos los casos a diámetros mayores. 


\section{ESTUDIO DEL CAMPO ELECTROMAGNÉTICO EN EL INTERIOR DE UN NW SEMICONDUCTOR EXCITADO CON UN HAZ LÁSER POLARIZADO}

no permiten su caracterización, ya que depositándolos en el substrato adecuado el acoplamiento electromagnético podría hacer que la señal fuese lo suficientemente intensa como para analizar su espectro Raman.

\subsection{Intensidad Raman en NWs sobre diferentes subs-}

\section{tratos}

Una de las anomalías detectadas cuando se realizó la caracterización Raman de los NWs de silicio en el capítulo 4 concernía a la intensidad observada en el espectro Raman de los NWs. Cuando se analizó el espectro Raman de un NW de silicio de $52 \mathrm{~nm}$ depositado en aluminio, se observó que tenía una intensidad Raman por unidad de volumen cientos de veces mayor que la intensidad Raman por unidad de volumen del silicio volúmico. Para ver si este efecto provenía del substrato, se realizaron experimentos con NWs de la misma muestra depositados en un substrato de germanio, observándose como la intensidad Raman por unidad de volumen de estos NWs también era mucho mayor que la correspondiente al silicio volúmico. Dado que el germanio es un semiconductor y el aluminio un metal, se asumió a priori que eran suficientemente diferentes como para eliminar al substrato de la ecuación, atribuyendo el efecto de la enorme señal Raman exclusivamente a características intrínsecas de los NWs. Sin embargo, y a la vista de los resultados mostrados en la figura 5.3, debemos reconsiderar esas conclusiones, puesto que si bien es cierto que el diámetro de cada NW juego un papel fundamental en la intensidad Raman, también lo es que la magnitud de esa señal depende del acoplamiento diámetrosubstrato para cada material y longitud de onda.

En concreto, y por pura casualidad, cuando se escogió el germanio y el aluminio como substratos diferenciales, no nos podíamos imaginar que ambos presentasen un comportamiento tan similar en el rango de diámetros que va desde 40 a $120 \mathrm{~nm}$ (ver figura 5.3 y 5.4). Esto es la causa de que al comparar los resultados obtenidos en ambos substratos, no pudiésemos distinguir claramente la enorme importancia que tiene el substrato en la intensidad Raman proveniente de los NWs.

En la figura 5.5 se muestran las distribuciones de $|E|^{2}$ para dos NWs de silicio 


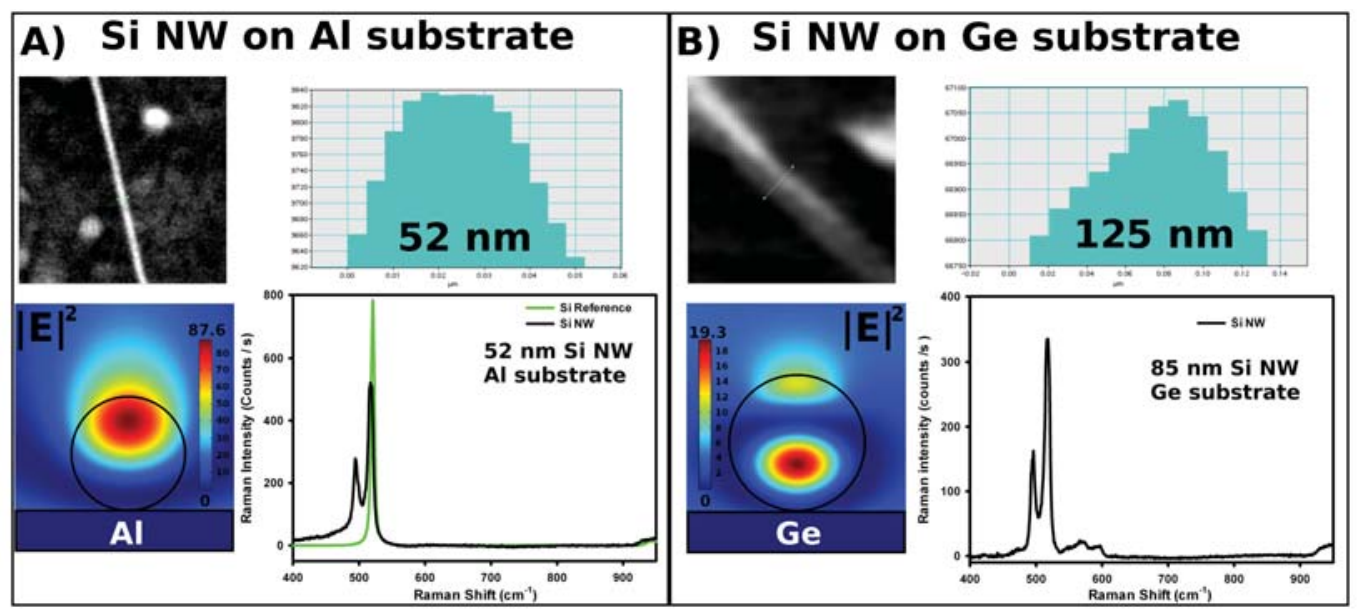

Figura 5.5: Distribución de $|E|^{2}$ y espectros Raman para dos NWs sobre aluminio y germanio. Puede observarse como para el NW de $52 \mathrm{~nm}$ depositado sobre aluminio el valor de $|E|^{2}$ es más de cuatro veces más intenso que para el NW de $125 \mathrm{~nm}$ depositado sobre germanio, lo que corresponde a un valor integrado de $|E|^{2} 1.6$ mayor en el NW de $52 \mathrm{~nm}$ que en el de $125 \mathrm{~nm}$.

depositados en aluminio y germanio respectivamente. La intensidad Raman integrada del NW depositado en aluminio es casi un $77 \%$ mayor que la intensidad Raman procedente del NW depositado en germanio, pese a que el volumen de éste es 5.56 veces mayor. En una visión macroscópica, uno esperaría que el NW con un mayor volumen tuviese una señal mucho más intensa; sin embargo, la distribución del campo eléctrico en el interior de los NWs y el efecto del substrato, hacen que el valor de $|E|^{2}$ integrado a la sección del NW de $52 \mathrm{~nm}$ sea 1.63 veces mayor que el valor de $|E|^{2}$ integrado a la sección del NW de $125 \mathrm{~nm}$. Dado que para para muestras bajo las mismas condiciones de excitación, (i.e. longitud de onda del campo electromagnético incidente), la intensidad Raman es proporcional a la intensidad total de $|E|^{2}$ (que es es el valor integrado en la sección de cada NW), podemos esperar un espectro cerca de un $60 \%$ más intenso para el NW más delgado. En la figura 5.5 puede observarse como experimentalmente se obtiene un comportamiento similar al predicho por el modelo teórico, obteniéndose un espectro entorno a un $\sim 77 \%$ más intenso para el NW más delgado depositado sobre aluminio. La ligera discrepancia frente al valor teórico predicho puede proceder de la diferencia en las condiciones de excitación de cada NW (e.g. diferentes valores de el ángulo que for- 


\section{ESTUDIO DEL CAMPO ELECTROMAGNÉTICO EN EL INTERIOR DE UN NW SEMICONDUCTOR EXCITADO CON UN HAZ LÁSER POLARIZADO}

ma cada NW con el eje de polarización del haz láser), que recordemos se suponen idénticas para poder comparar sus intensidades Raman únicamente a través de $|E|^{2}$ integrado a la sección.

Por otro lado, y bajo la simplificación que supone trabajar en 2D, el valor de la intensidad Raman obtenido de una muestra macroscópica es directamente proporcional al valor de $|E|^{2}$ integrado a la sección iluminada por el láser para cada longitud de onda. Por otro lado, en materiales en los que la luz del láser es fuertemente absorbida, como es el silicio, o el silicio-germanio para estas longitudes de onda, el volumen de material que contribuye a la señal Raman final va a depender del coeficiente de absorción para cada longitud de onda, siendo mayor cuanto menos absorbida sea la luz del láser. Dado que $|E|$ es es proporcional a la intensidad del láser, y que el decaimiento de la intensidad al penetrar en la muestra iluminada se puede evaluar mediante la aproximación dada por la ley de Beer-Lambert de la absorción; para la sección iluminada por el haz láser de distribución gaussiana se puede obtener una aproximación para el valor promedio de $|E|^{2}$ en una muestra volúmica a través de:

$$
|E|_{\text {Volumico }}^{2}=\int_{-\infty}^{\infty} \int_{0}^{\infty}\left\|(1-R) E(z, x, t) e^{- \text {alpha*z }}\right\|^{2} d x d z
$$

Donde $E(z, x, t)$ es el campo eléctrico mostrado en la ecuación 5.2, $R$ el coeficiente de reflexión $(\sim 0.38$ para el verde y $\sim 0.35$ para el rojo [18]) y $\alpha$ y el coeficiente de absorción para el silicio $\left(\alpha \sim 7.9863 \times 10^{5} \mathrm{~m}^{-1}\right.$ para el verde y $3.022 \times 10^{5}$ para el rojo [18]). Mediante esta aproximación, el valor de $|E|^{2}$ integrado a la sección iluminada por el láser de He-Ne en una muestra volúmica de silicio es $\sim 15$ veces mayor que el valor de $|E|^{2}$ integrado en la superficie del NW de $52 \mathrm{~nm}$ para esa longitud de onda e inclinación (El efecto de la orientación del NW puede verse en profundidad en la siguiente sección). Por su parte, la intensidad Raman integrada obtenida de los espectros Raman adquiridos con el láser de 632 nm, que es proporcional a este valor a través de la ecuación 5.1, es 15.5 veces mayor para el silicio de referencia que para el NW, en perfecto acuerdo con lo calculado teóricamente (ver imagen 5.6-B)). Si realizamos el mismo cálculo para el láser Nd:YAG, el valor $|E|^{2}$ integrado a la sección iluminada por el láser resulta ser 1.56 veces mayor que 


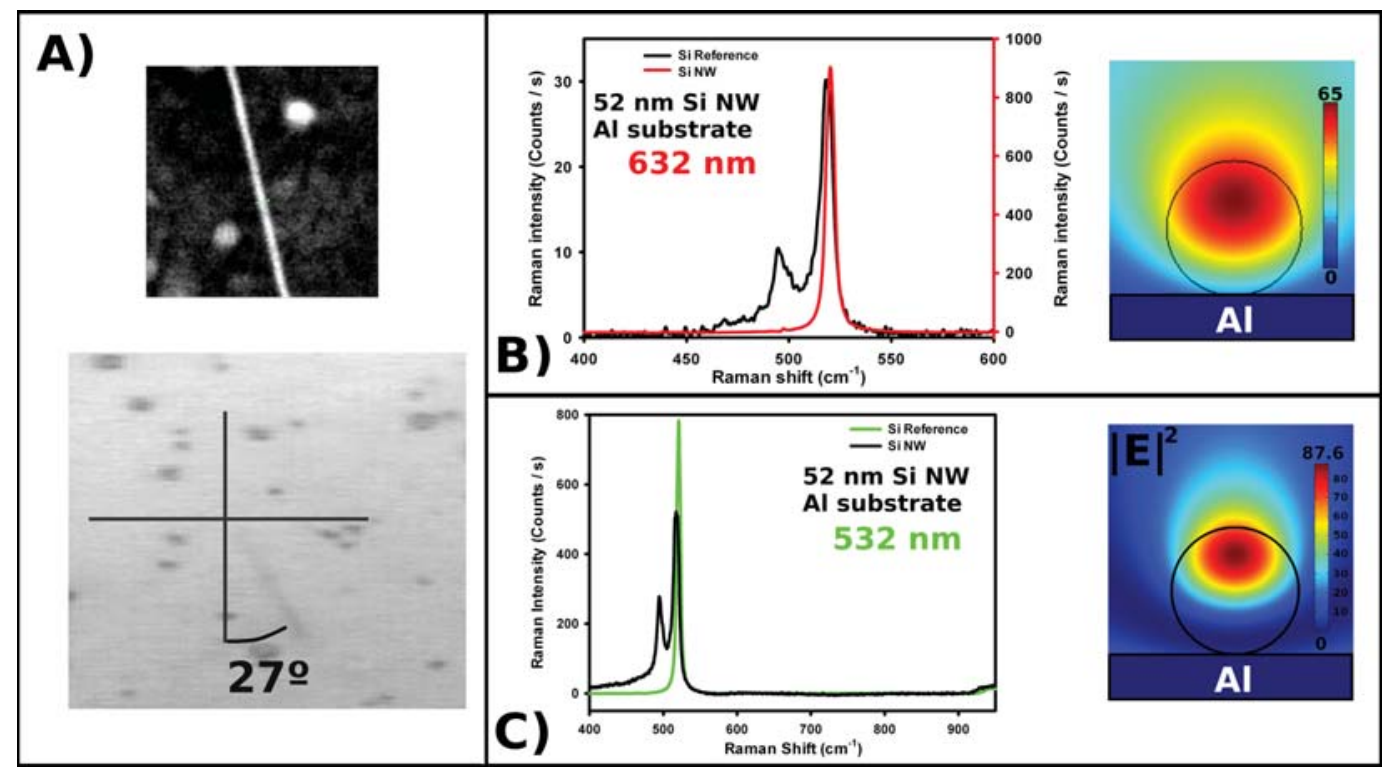

Figura 5.6: A) Imagen SEM ampliada del NW de silicio que aparece en la figura 4.16. Debajo Imagen procedente del microscopio óptico. B) Espectros Raman para el NW de $52 \mathrm{~nm}$ depositado sobre aluminio y para el silicio de referencia, ambos iluminados con láser He-Ne $(\lambda=632 \mathrm{~nm})$ (el silicio de referencia se lee en la escala derecha). Al lado la distribución de $|E|^{2}$ en el NW. C) Espectros Raman para el NW de $52 \mathrm{~nm}$ depositado sobre aluminio y para el silicio de referencia, ambos iluminados con láser Nd:YAG $(\lambda=532 \mathrm{~nm})$. Al lado la distribución de $|E|^{2}$ en el NW. Nótese que sólo son directamente comparables las intensidades de las medidas realizadas en muestras bajo las mismas condiciones de excitación, no siendo directamente comparables medidas realizadas con longitudes de onda diferentes. 


\section{ESTUDIO DEL CAMPO ELECTROMAGNÉTICO EN EL INTERIOR DE UN NW SEMICONDUCTOR EXCITADO CON UN HAZ LÁSER POLARIZADO}

el procedente del NW iluminado con el mismo láser; sin embargo, el cálculo procedente de las intensidades Raman de los espectros mostrados en la figura 5.6-C) arroja un valor para la intensidad del silicio de referencia de 0.998 veces el valor del NW (nótese que debido al calentamiento del NW, el pico Raman del silicio es más ancho que el pico del del substrato). Esta discrepancia entre el resultado teórico y el experimental para el láser de Nd:YAG nos indica que la intensidad Raman de los NWs cuando se iluminan con esta longitud de onda no depende directamente del valor de $|E|^{2}$ en el interior del NW. Por tanto, tiene que existir otro efecto contribuyendo en el scattering Raman para esa longitud de onda. Esta anomalía para el láser de $532 \mathrm{~nm}$ ha sido reportada por otros autores en [7].

Pese a la discrepancia encontrada para el láser Nd:YAG, a través de nuestro modelo teórico se demuestra que la enorme intensidad Raman observada en los espectros Raman de NWs individuales está relacionada con el substrato sobre el que se apoyan; por tanto, uno puede sintonizar el mejor substrato para cada NW de forma que se obtenga la mayor intensidad Raman posible. Esto puede ser un paso muy importante para conseguir obtener señales Raman mensurables en NWs de materiales caracterizados por tener una señal Raman muy débil. En nuestro caso sin este efecto introducido por el substrato no hubiera sido posible caracterizar los NWs con potencias tan bajas como $5 \mu \mathrm{W}$, ya que los tiempos de adquisición, ya largos de por sí para esta potencia de láser, hubiesen sido inabordables.

\subsection{Efectos de polarización en NWs de silicio.}

Dado que el diámetro de los NWs está muy por debajo de la longitud de onda del láser incidente, los NWs semiconductores, especialmente cuando sus diámetros son muy reducidos, presentan una estructura muy similar al la de una antena lineal [24]. Bajo esta aproximación, los NWs absorben la radiación incidente con un patrón dípolar proporcional a $\cos ^{2} \alpha$, siendo $\alpha$ el ángulo formado por el eje del NW y el campo eléctrico de la radiación electromagnética incidente [4]. Este efecto, de existir, debe manifestarse en la intensidad Raman procedente de NWs semiconductores. En este sentido, diversos autores han mostrado que el espectro Raman obtenido al iluminar NWs semiconductores con luz polarizada depende 
fuertemente de la posición de los NWs respecto al eje de polarización del haz incidente $[3,4,7,14,19-24]$. La mayoría de estos estudios concierne a NWs formados por semiconductores polares $[3,4,14,19-21,23]$, por lo tanto el efecto de la polarización en este tipo de estructuras es doble, ya que al posible efecto específico de la nanoestructura se suma la dependencia del tensor Raman con la polarización incidente [2]. En los NWs de materiales polares, los picos LO y TO aparecen separados y cada uno de ellos tiene una dependencia con la polarización de la luz incidente [24]. Esto hace que los patrones de intensidad observados en este tipo de NWs puedan resultar en complejas dependencias multipolares en función de la orientación del NW con respecto al eje de polarización y de sus diámetros [4, 14, 21]. Por su parte, tanto el silicio como el germanio presentan una estructura cúbica centrada en las caras, resultando en un tensor Raman 3-veces degenerado [26, 27], lo que implica que con independencia de la polarización del haz láser, el espectro Raman muestra un único pico principal correspondiente a los fonones LO y TO y siempre a la misma frecuencia. La dependencia de la intensidad del pico principal en el silicio con la polarización de la luz incidente fue estudiada mediante espectroscopia Raman polarizada por Nakashima et al, mostrando que en cristales perfectamente orientados la intensidad del espectro Raman depende de la polarización incidente [28]. Sin embargo, nuestros experimentos se realizan sin analizador, y por tanto no debería observarse una dependencia de la intensidad Raman por este motivo, al igual que no se manifiesta cuando se observa el espectro Raman del silicio de referencia para diferentes posiciones de la muestra. Sin embargo, para una configuración análoga a la de nuestro equipo, en la referencia [22] se muestra como en NWs de Silicio aparece una dependencia en la intensidad Raman en función de la orientación del NW respecto al eje de polarización de la luz incidente. Dado que este efecto no está directamente ligado al tensor Raman de los NWs de silicio, debe manifestarse en otros tipos de interacción entre la luz polarizada incidente y el NW. Este efecto se demuestra en la figura 5.6, donde se puede observar lo que le sucede a las imágenes obtenidas por microscopía óptica confocal (objetivo 100X) de un NW de silicio de $65 \mathrm{~nm}$ de diámetro, y depositado en aluminio, cuando el NW es paralelo a la dirección del campo eléctrico de la luz blanca incidente y cuando el NW es perpendicular a esta dirección. En la imagen SEM se observa que la muestra está compuesta por 3 NWs de silicio, estando dos de ellos a un ángulo cercano 


\section{ESTUDIO DEL CAMPO ELECTROMAGNÉTICO EN EL INTERIOR DE UN NW SEMICONDUCTOR EXCITADO CON UN HAZ LÁSER POLARIZADO}

a $90^{\circ}$ y el tercero a $32^{\circ}$ con respecto al tercero, que es el NW principal de nuestro análisis. Cuando este NW principal esta alineado con el campo eléctrico de la radiación incidente, luz blanca linealmente polarizada en este caso, se puede distinguir perfectamente el NW principal en la imagen obtenida por microscopia óptica confocal, mientras que el NW adyacente a $32^{\circ}$ sólo se vislumbra muy tenuemente. Cuando se rota $90^{\circ}$ la muestra, el patrón observado anteriormente cambia completamente, y el NW principal apenas es distinguible en esta configuración mientras que el NW adyacente a $32^{\circ}$ se aprecia perfectamente. Esta anisotropía introducida
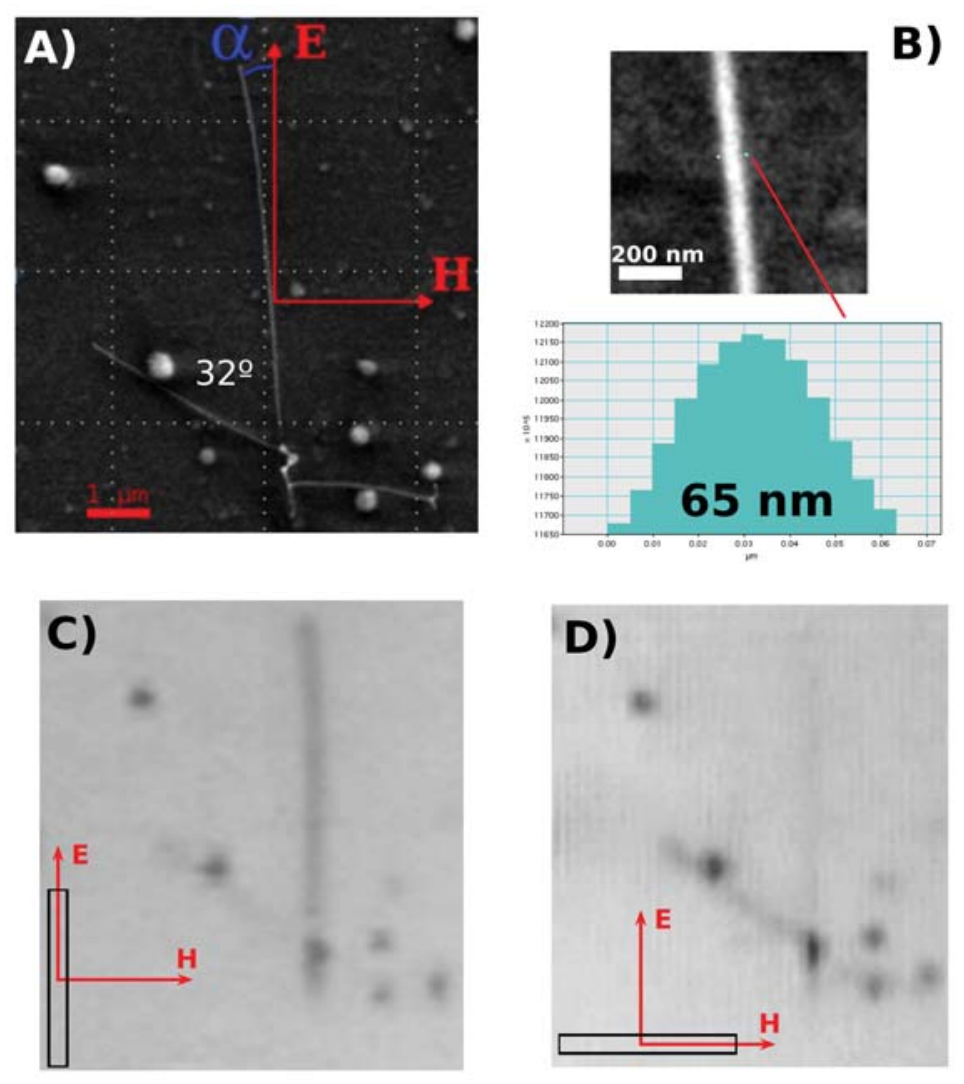

Figura 5.7: A) Imagen SEM de un conjunto de 3 NWs de silicio depositados sobre aluminio, el NW principal aparece próximo a $90^{\circ}$.B)- Diámetro del NW principal medido a partir de la imagen SEM. C)- Imagen obtenida con el microscopio confocal y el objetivo 100X con la muestra dispuesta de tal modo que el NW principal está alineado con el eje de polarización de la luz incidente. D)- Imagen obtenida al rotar la muestra $90^{\circ}$.

por la polarización del campo electromagnético incidente ha sido descrita a través 
de la diferencia entre las funciones dieléctricas del NW y el medio que lo rodea $[4,7,22]$. Por tanto, la existencia de un substrato como los analizados en las figuras 5.3 y 5.4 puede introducir un efecto muy relevante en la señal Raman observada en NWs de silicio cuando estos son iluminados con un haz láser linealmente polarizado. Este efecto se ha estudiado a través de nuestro modelo teórico FEM de la interacción electromagnética láser/NW/substrato, ya que permite definir cualquier orientación entre el eje del NW y eje de polarización del campo electromagnético incidente.

En la figura 5.7 se muestran los resultados de nuestra simulación para el valor de $|E|^{2}$ en el interior de NWs de distintos diámetros y en función del ángulo formado por su eje longitudinal y el eje de polarización del láser. Para diámetros pequeños $(50 \mathrm{~nm})$ y cuando el NW se encuentra perpendicular al eje de polarización, el campo eléctrico en el interior del NW es varios ordenes de magnitud menor que en la configuración paralela, tal y como se predice en [25] para estructuras cuasi-unidimensionales. Por tanto, el patrón que sigue el valor integrado de $|E|^{2}$ en función del ángulo $\alpha$ es prácticamente dipolar, con independencia del substrato y de la longitud de onda. Cuando el diámetro de los NWs se incrementa, el valor de $|E|^{2}$ aumenta cuando el NW se encuentra perpendicular al eje de polarización, alejándose su comportamiento del patrón dipolar dado por $\cos ^{2} \alpha$. Este efecto es más intenso para la longitud de onda de $532 \mathrm{~nm}$ que para la de $632 \mathrm{~nm}$, revelando una dependencia con la longitud de onda del campo electromagnético incidente. Los NWs que están rodeados por aire, tienen una menor dependencia $|E|^{2} \operatorname{con} \alpha$ que los depositados en aluminio, por lo que el substrato también juega un papel fundamental en este comportamiento anisotropíco de $|E|^{2}$. A la vista de estos resultados, y dada la dependencia de la intensidad Raman con $|E|^{2}$, cabe esperar que el espectro Raman obtenido en NWs de silicio con diámetros menores a $90 \mathrm{~nm}$ presenten un patrón casi dipolar en la intensidad para las dos longitudes de onda de los láseres He-NE y Nd:YAG, con su cintura mas abierta cuanto mayor es el diámetro y menor la longitud de onda incidente. Resulta curioso el resultado obtenido para un NW de silicio de $125 \mathrm{~nm}$, puesto que en este caso cuando se ilumina con el haz de $532 \mathrm{~nm}$, el resultado de que el NW esté sobre un substrato de aluminio ó rodeado por aire arroja el mismo comportamiento, mientras que si se ilumina con el láser He-Ne, el efecto del substrato es determinante, obteniéndose un patrón 


\section{ESTUDIO DEL CAMPO ELECTROMAGNÉTICO EN EL INTERIOR DE UN NW SEMICONDUCTOR EXCITADO CON UN HAZ LÁSER POLARIZADO}
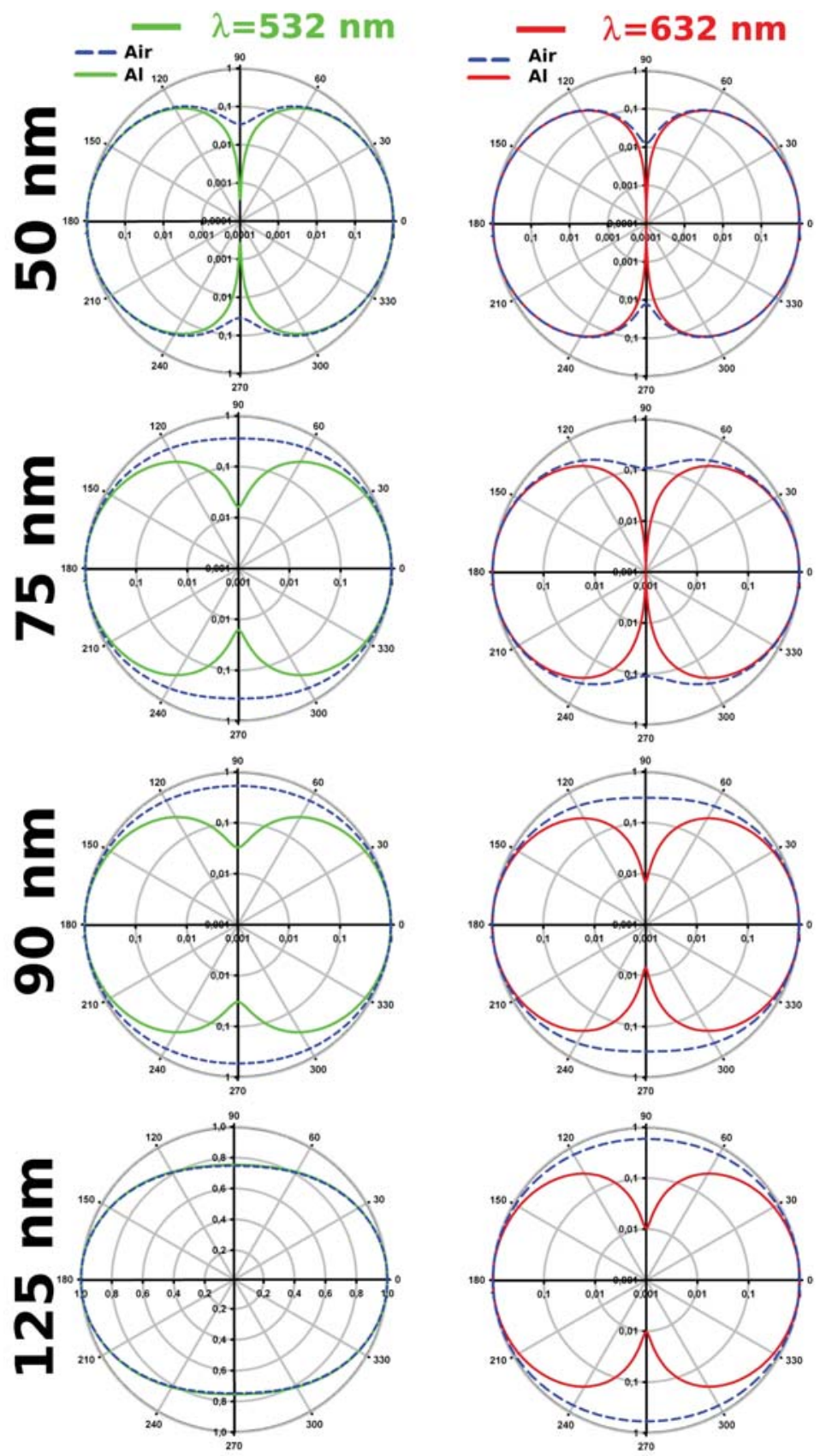

Figura 5.8: Valor de $|E|^{2}$ integrado en función del ángulo que forman NWs de distintos diámetros con el eje de polarización del láser incidente. El estudio se ha realizado para dos longitudes de onda diferentes, $532 \mathrm{~nm}$ y $632 \mathrm{~nm}$. Nótese que todos los gráficos son Log-Log salvo el perteneciente al NW de $125 \mathrm{~nm}$ iluminado por el haz de 532 nm. 
pseudo-dipolar.

A la vista de la figura 5.7, uno podría pensar que para diámetros por encima de $125 \mathrm{~nm}$ el patrón dipolar desaparecerá cuando se utiliza el láser Nd:YAG, de tal modo que $|E|^{2}$ sea constante con independencia del valor de $\alpha$. Sin embargo, el acoplamiento electromagnético entre el láser, el NW, y substrato es más complejo que está extrapolación. A modo de ejemplo, en la figura 5.8 se muestra como pueden aparecer diferentes patrones de $|E|^{2}$ en función de $\alpha$ solamente modificando el material del substrato sobre el que se deposita un NW de silicio de $187 \mathrm{~nm}$ de diámetro. Para este NW y a la vista de la figura 5.7, cabría esperar que el valor de $|E|^{2}$ cuando el NW está depositado en aluminio sea independiente de $\alpha$; sin embargo, lo que aparece es un patrón en el que $|E|^{2}$ es mucho mayor cuando el eje del NW se encuentra perpendicular al eje de polarización del láser; es decir, el comportamiento inverso a lo visto en NWs de diámetros más pequeños. Este comportamiento se invierte cambiando el substrato por germanio u oro, poniendo de manifiesto el papel esencial que juega el substrato en la interacción láser/NW/substrato.

\subsubsection{Efectos de polarización el los espectros Raman adquiridos en NWs de silicio}

La dependencia de $|E|^{2}$ con el ángulo $\alpha$, formado por el eje del NW y el eje de polarización del haz láser incidente, tiene que traducirse en una dependencia de la intensidad Raman procedente de los NWs de silicio al variar $\alpha$.

Para comprobar este efecto, se ha adquirido el espectro Raman del NW principal de $65 \mathrm{~nm}$ de diámetro mostrado en la figura 5.6, rotando la muestra bajo el haz láser de forma controlada para obtener los espectros en función de $\alpha$. El resultado cuando se ilumina con el láser de He-Ne de $632 \mathrm{~nm}$ de longitud de onda se muestra en la figura 5.9. En 5.9-A) se observa como la intensidad de espectro Raman efectivamente es dependiente del ángulo que se ha girado la muestra, y por tanto se muestra como decae la intensidad Raman al rotar el NW respecto al eje de polarización del haz láser. En 5.9-B) se muestra el mapa polar normalizado de la intensidad Raman de los espectros adquiridos al rotar el NW de $65 \mathrm{~nm}$ respecto al eje de polarización del 


\section{ESTUDIO DEL CAMPO ELECTROMAGNÉTICO EN EL INTERIOR DE UN NW SEMICONDUCTOR EXCITADO CON UN HAZ LÁSER POLARIZADO}
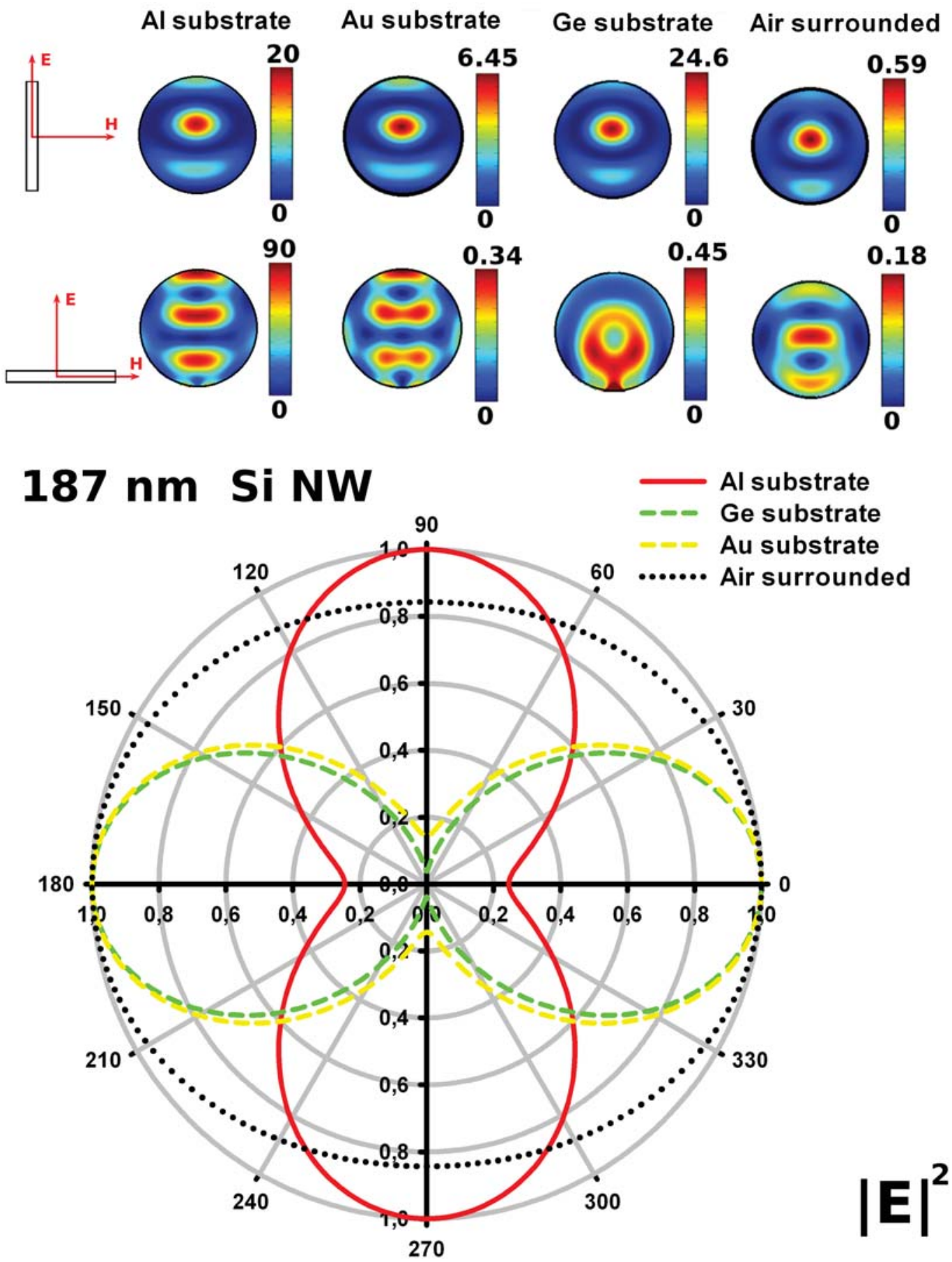

Figura 5.9: $|E|^{2}$ integrado en un NW de silicio de $187 \mathrm{~nm}$ de diámetro. Pueden observarse las diferentes distribuciones de $|E|^{2}$ en el interior del nanohilo en función del substrato en el que está depositado el NW. El gráfico polar muestra el valor de $|E|^{2}$ en función de $\alpha$ para cada substrato. Nótese que enorme efecto que introduce el substrato en la dependencia de $|E|^{2}$ con $\alpha$ llegándose a invertir el comportamiento pseudo-dipolar cuando el substrato es aluminio. 
láser incidente. El patrón que aparece es idéntico al patrón calculado teóricamente para $|E|^{2}$ en las mismas condiciones y para el mismo NW. Ésto está en perfecto acuerdo con el resultado obtenido en la sección anterior para otro NW diferente de silicio, donde se demostraba que la intensidad Raman relativa entre el substrato y el NW cuando son excitados por la misma fuente electromagnética era proporcional únicamente a $|E|^{2}$. Por tanto, todo parece indicar que el único efecto causante de que la intensidad Raman decaiga al rotar el NW es la dependencia predicha por nuestro modelo del campo eléctrico inducido en el NW con $\alpha$. Cuando se repite el experimento para el mismo NW, pero cambiando el láser de He-Ne por el Nd:YAG de $532 \mathrm{~nm}$, se observa como la intensidad Raman de los espectros también depende fuertemente del ángulo $\alpha$ que caracteriza la posición del NW respecto al eje de polarización del haz láser (Ver figura 5.10).

Cuando se representa la intensidad de estos espectros en un mapa polar, llama la atención que la distribución de intensidades Raman en función del ángulo $\alpha$ tiene una forma muy aguda (Ver figura 5.11-A)). De hecho, cuando se compara con la distribución de $|E|^{2}$ en función del ángulo calculada para este NW y en estas condiciones, se observa que a diferencia de lo visto para el láser de He-Ne en este mismo NW, la intensidad Raman no sigue el mismo patrón que $|E|^{2}$ (ver figuras 5.11-B) y 5.11-C) ), sino que la intensidad Raman es mucho más dependiente del ángulo formado por el NW y el eje de polarización que el valor de $|E|^{2}$ calculado. Precisamente, un resultado similar a este ha sido reportado por Lopez et al. en la referencia [7] al iluminar un nanocono de silicio con la longitud de onda de $532 \mathrm{~nm}$. En esté artículo argumentan que, en concreto para esta longitud de onda, la intensidad Raman se ve reforzada por el campo eléctrico correspondiente a la emisión Raman, sino que es proporcional a $|E|^{2} *\left|E_{\text {scatteringRaman }}\right|^{2}$. Ésto está en perfecto acuerdo con la discrepancia encontrada en la sección anterior para el láser Nd:YAG. Recordemos que el NW iluminado por el láser 532 en la figura 5.6 mostraba una intensidad Raman relativa al silicio de referencia mayor que la correspondiente por la relación dada por $|E|^{2}$, y que por lo tanto se intuía que otro efecto estaba contribuyendo al scattering Raman. En la figura 5.11-C), se muestra en un mapa polar Log-Log la distribución de intensidades. Puede observarse como pese a que las distribución calculada para $|E|^{2}$ no describe bien la dependencia de la intensidad 


\section{ESTUDIO DEL CAMPO ELECTROMAGNÉTICO EN EL INTERIOR DE UN NW SEMICONDUCTOR EXCITADO CON UN HAZ LÁSER POLARIZADO}
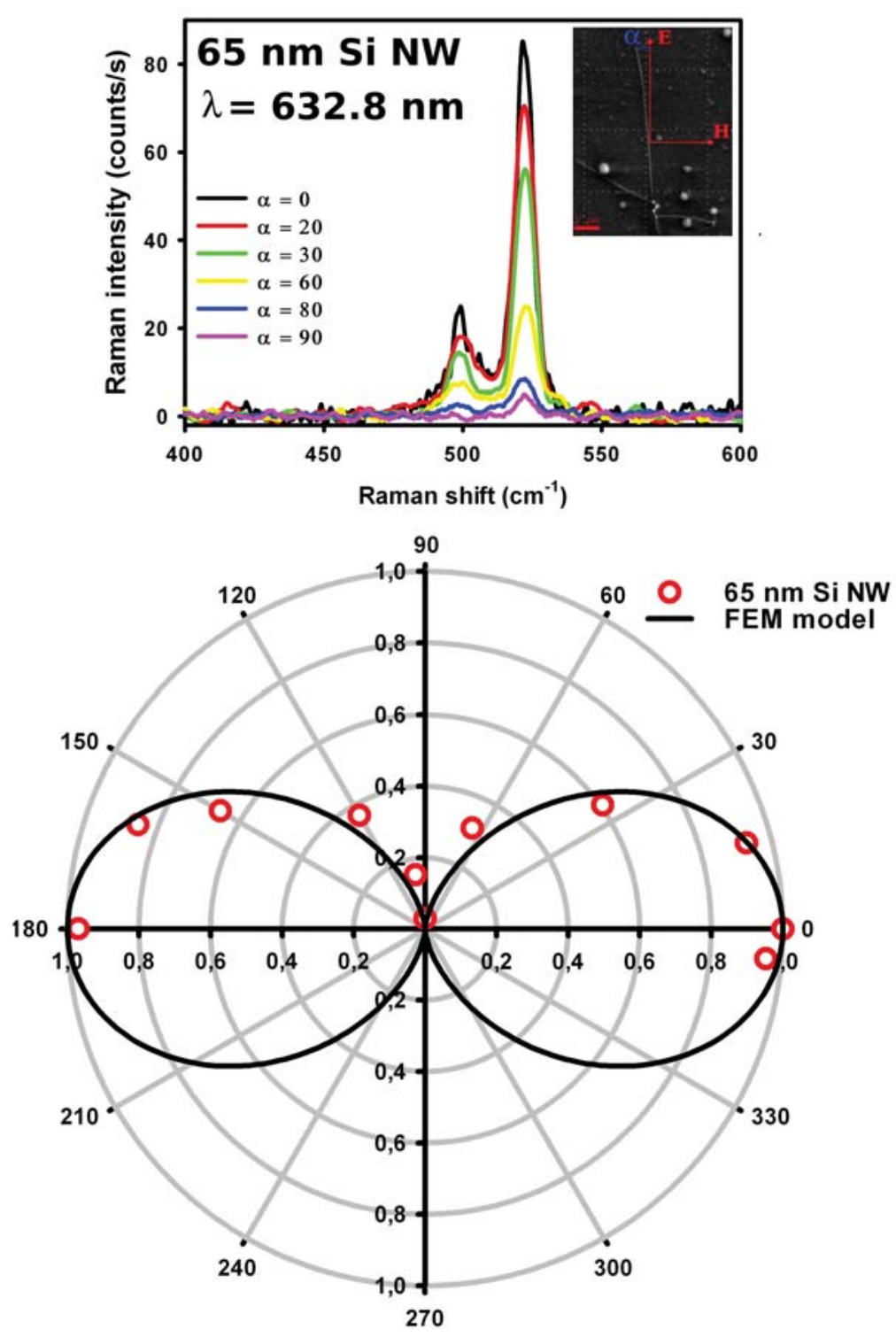

Figura 5.10: A)- Espectros Raman obtenidos en el NW principal de la figura 5.6 en función del ángulo $\alpha$ cuando se ilumina el NW con el láser He-Ne de $632.8 \mathrm{~nm}$ de longitud de onda. B) Patrón de intensidades Raman normalizadas obtenidas al girar la muestra para valores de $\alpha$ entre $0^{\circ}$ y $180^{\circ}$. Nótese como aparece el comportamiento predicho por el modelo teórico del acoplamiento electromagnético 


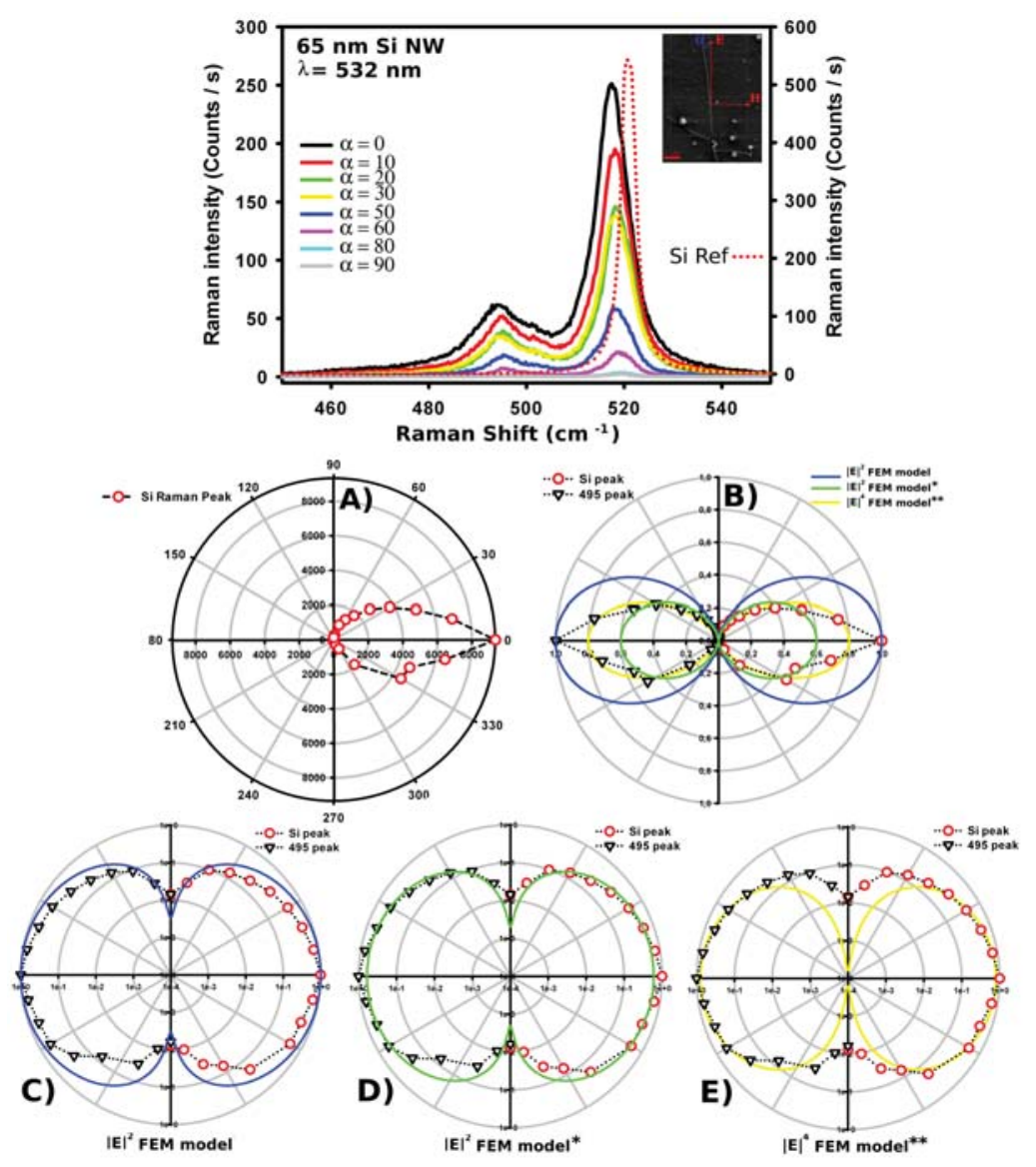

Figura 5.11: Espectros Raman obtenidos en el NW principal de la figura 5.6 en función del $\alpha$ cuando se ilumina el NW con el láser Nd:YAG de $532.8 \mathrm{~nm}$ de longitud de onda. A) Patrón de intensidad Raman del pico principal del silicio en función de $\alpha \in\left[0^{\circ}, 180^{\circ}\right] \cup\left[270^{\circ}, 360^{\circ}\right]$. B) Patrón de intensidad Raman del pico principal del silicio en función de $\alpha \in\left[0^{\circ}, 180^{\circ}\right] \cup\left[270^{\circ}, 360^{\circ}\right]$ y del pico 495 en $\alpha \in\left[180^{\circ}, 270^{\circ}\right]$. Nótese como el patrón de intensidades experimentales difiere del patrón obtenido para $|E|^{2}$ en las mismas condiciones experimentales. C) Representación en escala Log-Log del patrón mostrado en B) y el valor calculado para $|E|^{2}$ normalizado. D) Representación en escala Log-Log del patrón mostrado en B) y mejor ajuste utilizando el patrón de teórico de $|E|^{2}$. F) Representación en escala Log-Log del patrón mostrado en B) y mejor ajuste a $|E|^{4}$ como la propuesta en [4] y [7] 


\section{ESTUDIO DEL CAMPO ELECTROMAGNÉTICO EN EL INTERIOR DE UN NW SEMICONDUCTOR EXCITADO CON UN HAZ LÁSER POLARIZADO}

Raman con $\alpha$, para valores pequeños de $\alpha$ si que se obtiene de forma precisa el comportamiento de la intensidad Raman, puesto que la abertura del patrón de intensidades a $90^{\circ}$ de $|E|^{2}$ y de la intensidad Raman tienen un valor muy similar. Al ir aumentando el valor de $\alpha$, el patrón de la intensidad Raman se va distorsionando, ajustando a otro tipo de dependencia con el ángulo, lo que indica que el efecto que desvía la intensidad Raman de su dependencia normal con $|E|^{2}$ aumenta al disminuir $\alpha$.

\subsection{Interacción electromagnética láser-NW y efectos de polarización en NWs de silicio-germanio}

El modelo teórico para la interacción láser-NW-substrato también puede ser aplicado a los NWs de silicio-germanio, pudiendo incorporar además los efectos que una coraza rica en germanio pueda aportar. La existencia de está capa rica en germanio deformaba los espectros Raman obtenidos en los NWs de tal manera que estos espectros aparecían como la composición de dos aportaciones bien diferencias, una que se correspondía con la composición estequiométrica de los NWs y caracterizada por el pico $\mathrm{Si}-\mathrm{Si}$ del espectro, y otra muy rica en germanio visible a través del pico Ge-Ge. De esta forma, los espectros obtenidos eran muy similares a los procedentes de NWs "core - shell" formados por una capa muy gruesa de germanio puro y un núcleo más pequeño de silicio-germanio. Estos espectros presentaban dos incógnitas difíciles de explicar a través de lo que se sabe de los NWs, lo que nos dejaba dos preguntas abiertas:

1. Dada la baja cantidad de germanio en la aleación, y por tanto la pequeña cantidad de germanio segregado en superficie, ¿cómo es posible que la componente rica en germanio pueda observarse con tanta intensidad?

2. ¿Cómo es posible que la componente rica en germanio de los espectros Raman sea sensible a la posición que ocupa el NW en área de iluminada por el Láser si cuando se hace un barrido transversal se está iluminando la misma zona del NW? 


\subsection{Interacción electromagnética láser-NW y efectos de polarización en NWs de silicio-germanio}

Para tratar de aportar luz a estas cuestiones, se ha simulado como es el campo eléctrico en el interior del NW bajo las mismas condiciones experimentales utilizadas en la adquisición de estos espectros Raman. El resultado de estas simulaciones para un NW de $85 \mathrm{~nm}$ de $S i_{85} G e_{15}$ puede verse en la figura 5.11. El NW se encuentra depositado en aluminio y se ilumina con el láser Nd:YAG de $532 \mathrm{~nm}$, mientras que para la simulación se ha supuesto una coraza de $4 \mathrm{~nm}$ de grosor de germanio puro. Las imágenes de la izquierda muestran la distribución de $|E|^{2}$ en el interior del NW, y revelan que para esta configuración análoga a la experimental, la distribución de $|E|^{2}$ es muy inhomogénea, concentrándose principalmente en dos zonas muy próximas a la superficie, mientras que la mayor parte del interior del NW está caracterizado por unos valores de $|E|^{2}$ al menos un orden de magnitud más bajos que los observados en estas zonas próximas a la superficie. Dado que la intensidad Raman es proporcional a $|E|^{2}$, lo que nos muestran estas figuras es que la señal Raman procede principalmente de estas dos zonas, mientras que el interior del NW contribuye de forma más débil a la señal final. Como sabemos que nuestros NWs tienen una pequeña capa próxima a la superficie muy rica en germanio (incluso germanio puro, ver figura 4.31), esta distribución de $|E|^{2}$ en los NWs magnifica la señal que proviene de este pequeño volumen de NW rico en germanio, tal y como muestran los espectros Raman mostrados en 5.11. Esta es también la razón de que al determinar la composición de los NWs a través de la posición del pico Ge-Ge se obtuviese siempre un valor mucho más alto de germanio en la aleación que cuando se obtenía del pico Si-Si o del EDX.

Por otro lado, el pico 495 suplementario que aparece en los NWs de silicio y siliciogermanio podría tener una explicación similar si está relacionado con algún efecto localizado en la superficie. Para los diámetros analizados experimentalmente la distribución de $|E|^{2}$ siempre tiene un máximo en las proximidades de la superficie (ver figuras 5.5 y 5.11), mientras que gran parte del volumen del NW apenas contribuye a la señal Raman; por tanto, si el pico 495 es un efecto de superficie, en estos NWs siempre se vera mucho más intenso que si $|E|^{2}$ estuviese distribuido de una forma más homogénea en el interior de la muestra.

La imagen 5.11 también encierra la respuesta a por qué la componente segregada de los espectros Raman depende de la posición que ocupa el NW en el área de iluminación. Dada la inhomogeneidad de la distribución de $|E|^{2}$ en el interior del 


\section{ESTUDIO DEL CAMPO ELECTROMAGNÉTICO EN EL INTERIOR DE UN NW SEMICONDUCTOR EXCITADO CON UN HAZ LÁSER POLARIZADO}
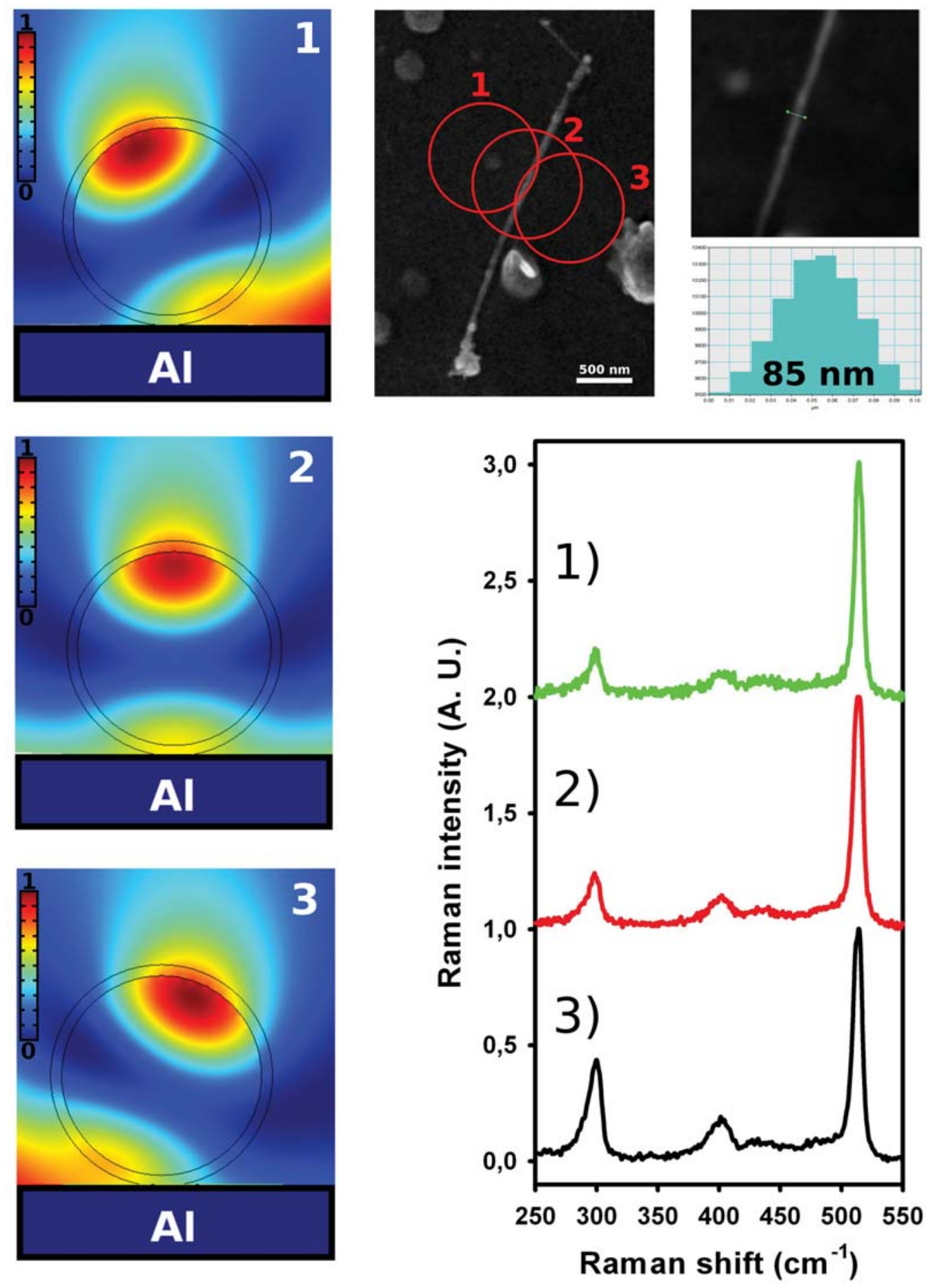

Figura 5.12: Distribución de $|E|^{2}$ en el interior de un NW de $85 \mathrm{~nm}$ de diámetro como el mostrado en la imagen SEM que acompaña a la figura. Las figuras numeradas corresponden a la posición que ocupa el NW en el haz láser en pasos de $500 \mathrm{~nm}$, que son los puntos donde se han adquirido los espectros Raman numerados de idéntica forma (ver esquema de la posición que ocupa el NW en la zona iluminada en la imagen SEM). Nótese como la distribución de $|E|^{2}$ se concentra en distintas partes del NW en función de la posición que ocupa el hilo en el área iluminada por el haz láser 


\subsection{Interacción electromagnética láser-NW y efectos de polarización en NWs}

de silicio-germanio

NW, la señal Raman procede principalmente de una zona muy localizada y próxima a la superficie, y este área localizado depende de la posición del NW en el área iluminada por el haz gaussiano del láser. Por otro lado, la zona rica en germanio no tiene por que ser una corteza homogénea, y la cantidad de germanio segregado puede variar en la zona próxima a la superficie (ver figura 4.31, en ella se aprecia esta inhomogeneidad de la composición en la sección del NW). Estos efectos combinados justifican que la componente Raman procedente de la zona con germanio segregado tenga diferente forma según la posición que ocupa el NW en el área de iluminación, puesto que la señal Raman de la zona segregada procede de diferentes zonas de la superficie donde la cantidad de germanio segregado puede ser muy diferente.

\subsubsection{Efectos de Polarización en NWs de silicio-germanio}

Al igual que los espectros Raman de los NWs de silicio, los cuales muestran una fuerte dependencia con el ángulo que forman los NWs con el eje de polarización del haz láser incidente, los NWs de silicio-germanio también son susceptibles de presentar el mismo efecto. Para estudiar esta posible dependencia, sobre el NW de $S i_{85} G e_{15}$ mostrado en la figura 5.12 se ha realizado el mismo experimento Raman realizado en los NWs de silicio. Es decir, el NW de silicio de $85 \mathrm{~nm}$ de grosor y depositado en aluminio, se ha ido rotando bajo el haz láser Nd:YAG. En este caso se han utilizado dos potencias del láser Nd:YAG, 500 y $50 \mu \mathrm{W}$ para probar si existe algún tipo de dependencia con este parámetro.

Los espectros obtenidos en cada caso pueden observarse en a figura 5.12, donde se muestra como la intensidad Raman de los NWs de silicio-germanio también es muy dependiente del ángulo $\alpha$ formado por el eje del NW y el eje de polarización del haz láser incidente. Este mismo experimento se ha reproducido teóricamente en nuestro modelo FEM tomando las mismas condiciones experimentales y bajo supuestos diferentes, uno en el que el NW de silicio-germanio es homogéneo en su totalidad y otro en el que se asume una coraza de germanio puro en la superficie de $4 \mathrm{~nm}$. El patrón que se obtiene en ambos casos para $|E|^{2}$ integrado en la sección del NW en función $\alpha$ se ha representado en la figura 5.12, donde también aparecen representadas las intensidades Raman integradas obtenidas experimentalmente. En 
este caso, y a diferencia de lo observado para los NWs de silicio iluminados por el láser Nd:YaG, la intensidad Raman sigue perfectamente el comportamiento marcado por $|E|^{2}$, demostrándose además que la correspondencia entre el patrón de $|E|^{2}$ y el de la intensidad Raman es mejor cuando se asume la existencia de la coraza de germanio segregado.

Por tanto, parece que la dependencia con $\alpha$ en este NW de silicio-germanio se debe completamente a como es el campo eléctrico en el interior del NW, al igual que se observaba para el NW de silicio iluminado con el láser He-Ne. 

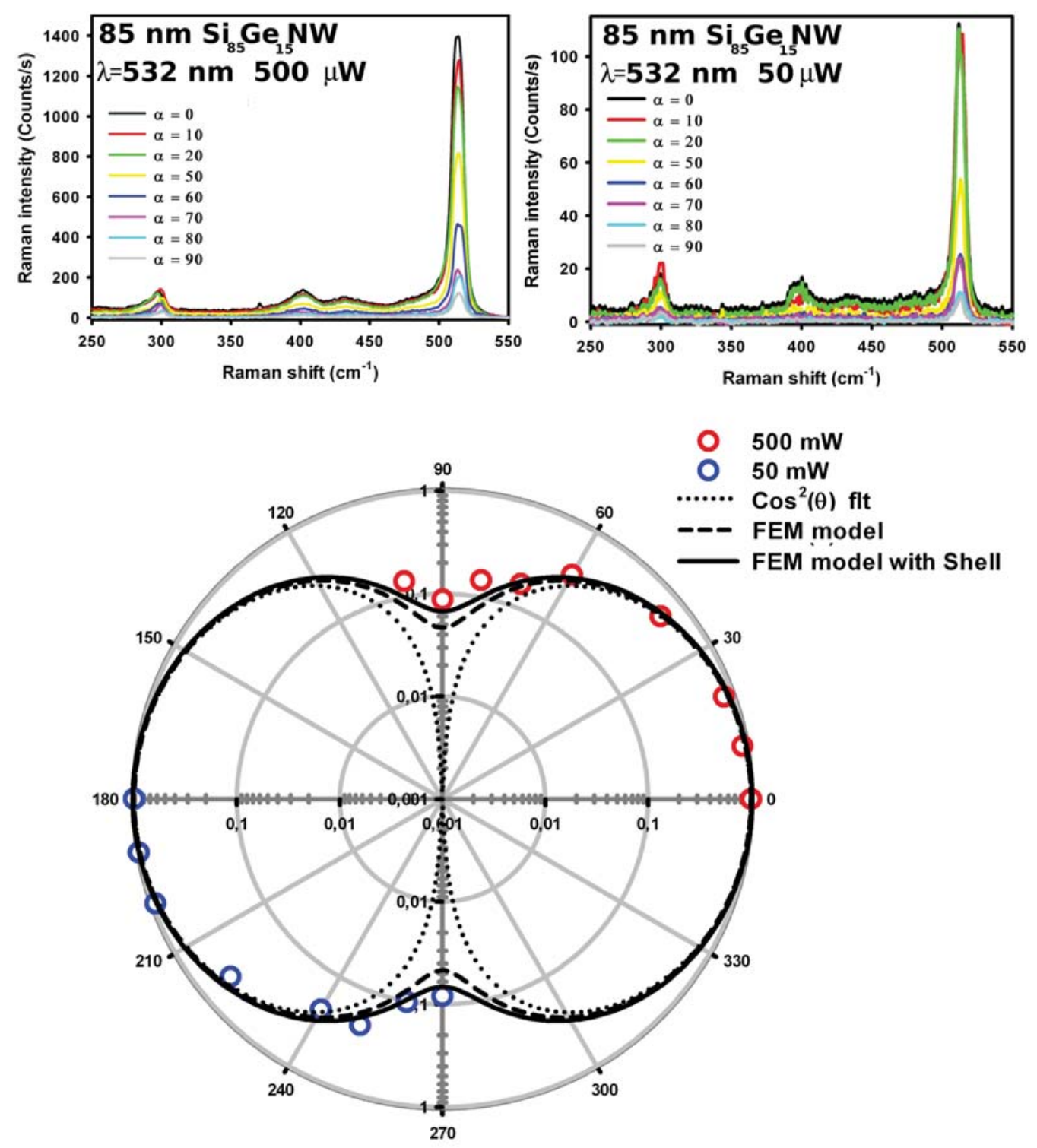

Figura 5.13: Serie de espectros Raman adquiridos para el NW de silicio-germanio de la figura 5.12 para dos potencias distintas del láser Nd:YaG en función del ángulo formado por el eje del NW y el eje de polarización. En el mapa polar puede observarse la dependencia de la intensidad del pico Si-Si con dicho ángulo, mostrándose también el patrón de $|E|^{2}$ simulado para este NW en las mismas condiciones experimentales. Nótese como la intensidad Raman obedece el patrón marcado por $|E|^{2}$, obteniéndose un mejor ajuste cuando se considera que el NW tiene una pequeña coraza de silicio segregado $(4 \mathrm{~nm})$. 



\subsection{Bibliografía}

[1] Derek L. A.The Raman Effect: A Unified Treatment of the Theory of Raman Scattering by Molecules, Ch 3, John Wiley \& Sons, Chichester, 2002.

[2] Nafie L. A. Theory of Raman Scattering, Handbook of Raman Spectroscopy, Edit Lewis, R. I and Edwards H. G. M.Mercel Dekker, New York, 2001.

[3] Xiong Q, Chen G, Gutierrez H. R, Eklund P. C. Raman scattering studies of individual polar semiconducting nanowires: phonon splitting and antenna effects, Appl. Phys. A, 2006;85, 299.

[4] Chen G, Wu J, Lu Q, Gutierrez H. R, Xiong Q, Pellen M. E., Petko J. S, Werner D. H, Eklund P. C. Optical Antenna Effect in Semiconducting Nanowires, Nano Lett., 2008;8, 2008.

[5] Cao L, Fan P, Brongersma M. L, Optical Coupling of Deep-Subwavelength Semiconductor Nanowires, Nano Lett. 2011;11, 1463.

[6] Cao L, Nabet B, Spanier J. E. Enhanced Raman Scattering from Individual Semiconductor Nanocones and Nanowires, Phys. Rev. Lett. 2006;96, 157402.

[7] Lopez F. J, Hyun J. K, Givan U, Kim I. S, Holsteen A. L, Lauhon L. J. Diameter and Polarization-Dependent Raman Scattering Intensities of Semiconductor Nanowires, Nano. Lett., 2012;12, 2266.

[8] Choi D.H, Hoefer W. J. R. The Finite-Difference-Time-Domain Method and its Application to Eigenvalue Problems, IEEE Transactions on Microwave Theory and Techniques, 1986;34, 1464.

[9] Yurkina M.A, Hoekstraa A. G. The discrete dipole approximation: An overview and recent developments, Journal of Quantitative Spectroscopy and Radiative Transfer, 2007;106, 558.

[10] Svelto O, David D, Hanna C. Principles of lasers, 5th edition, Springer, Heilderberg, 2010.

[11] Saleh B. E. A, Teich M. C. Fundamentals of Photonics Ch. 5, John Wiley \& Sons, 1991.

[12] Berry M. V. The electric and magnetic polarization singularities of paraxial waves, J. Opt. A: Pure Appl. Opt. 2004;6, 475.

[13] Gouy L. G, "Sur une propriete nouvelle des ondes lumineuses", C. R. Acad. Sci. Paris 1890;110, 1251. 


\section{ESTUDIO DEL CAMPO ELECTROMAGNÉTICO EN EL INTERIOR DE UN NW SEMICONDUCTOR EXCITADO CON UN HAZ LÁSER POLARIZADO}

[14] Hsu H. C., Hsu G. M, Lai Y. S, Feng Z. C, Tseng S. Y, Lundskog A, Forsberg U, Janzén E, Chen K. H, Chen L. C, Polarized and diameter-dependent Raman scattering from individual aluminum nitride nanowires: The antenna and cavity effects, Appl. Phys. Lett. 2012;101, 121902.

[15] Berenguer J. P. A perfectly matched layer for the absorption of electromagnetic waves, Journal of Computational Physics 1994;114, 185.

[16] Bereguer J. P. Perfectly Matched Layer (PML) for Computational Electromagnetics, Morgan \& Claypol, Arizona State University, 2007.

[17] Kerker M. The scattering of light and other electromagnetic radiation; Ch.3 and Ch.8. (Academic Press, New York 1969).

[18] Datos procedentes de la base de datos pertenecientes a Sopra S.A., Francia.

[19] Qi H, Rendell R. W, Glembocki O. J, Prokes S. M. Polarization Dependence of Surface Enhanced Raman Scattering on a Single Dielectric Nanowire, Journal of Nanomaterials, 2012;2012, 946868.

[20] Schäfer-Nolte E. O, Stoica T, Gotschke T, Limbach F, Sutter E, Sutter P, Calarco R. Highly polarized Raman scattering anisotropy in single GaN nanowires [21] Zardo I,Conesa-Boj S, Peiro F, Morante J. R, Arbiol J, Uccelli E, Abstreiter G, Fontcuberta i Morral A. Raman spectroscopy of wurtzite and zinc-blende GaAs nanowires: Polarization dependence, selection rules, and strain effects, Phys. Rev. B 2009;80, 245324.

[22] Frechétte J, Carraro C. Diameter-dependent modulation and polarization anisotropy in Raman scattering from individual nanowires, Phys. Rev. B 2006;74, 161404.

[23] Livneh T, Zhang J, Cheng G, Moskovits M. Polarized Raman scattering from single GaN nanowires, Phys. Rev B, 2006;74, 035320.

[24] Kim S. K, Day R. W, Cahoon J. F, Kempa T. J, Song K. D, Park H. G, Lieber C. M. Tuning Light Absorption in Core/Shell Silicon Nanowire Photovoltaic Devices through Morphological Design, Nano Lett. 2012;12, 4971.

[25] Ajiki H, Ando T. Aharonov-Bohm effect on magnetic properties of carbon nanotubes, Physica B 1996;216, 358.

[26]Loudon R, The Raman effect in crystals, Adv. Phys. 1964;13, 423.

[27] de Wolf I. Micro-Raman spectroscopy to study local mechanical stress in silicon integrated circuits, Semicond. Sci. Technol. 1996;11, 139. 


\subsection{Bibliografía}

[28] Nakashima S, Hangyo M. Characterization of Semiconductor Materials by Raman Microprobe, IEEE Journal of Quantum Electronics, 1989;25, 965 

It is better to recognise that we are in darkness than to pretend that we can see the light.

Hedley Bull

\section{Resumen y Conclusiones}

Los NWs semiconductores son estructuras de baja dimensión muy prometedoras para la construcción de los futuros nanodispositivos enfocados hacia múltiples ámbitos de aplicación tecnológica. Las reducidas dimensiones de estas estructuras les proporcionan unas características únicas, substancialmente diferentes a sus equivalentes volúmicos. Sin embargo, su implantación tecnológica está condicionada a conseguir que sus propiedades físicas puedan ser controladas, de modo que muestren un comportamiento homogéneo frente a un determinado estímulo. Por tanto avanzar en la comprensión de sus propiedades fundamentales, tanto a través de estudios teóricos como experimentales, es una tarea fundamental para la implantación tecnológica de estas estructuras.

En el primer capítulo de esta tesis, se ha hecho una aproximación teórica a las propiedades de transporte térmico de los NWs de silicio y silicio-germanio. La conductividad térmica de estas estructuras es mucho menor que la de sus homólogos volúmicos, y a diferencia de estos, la conductividad térmica no es una característica intrínseca del material, sino que depende de diferentes características propias de la morfología de la estructura. Para subsanar la falta de información experimental existente sobre esta magnitud física característica de los NWs de silicio y silicio-germanio, se ha desarrollado un completo modelo teórico capaz de predecir la conductividad térmica en NWs semiconductores de forma precisa. Este modelo 


\section{RESUMEN Y CONCLUSIONES}

incorpora todos los parámetros intrínsecos a la conductividad térmica de los NWs, así como los parámetros extrínsecos como el diámetro y la superficie, propios de cada NW. Este modelo permite predecir de forma precisa la conductividad térmica de NWs de silicio lisos en todo el rango de temperaturas, y en un amplio rango de diámetros, estando incluidos los NWs de diámetros más típicos en los estudios relacionados con estas estructuras. Cuando no se conocen las características de la superficie de los NWs, nuestro modelo permite obtener la conductividad térmica de forma precisa a cualquier temperatura con sólo conocer un único valor experimental de la conductividad térmica, simplificando mucho la caracterización de esta propiedad, siempre difícil. En el caso de disponer de información sobre las características de la superficie, i.e. rugosidad media y longitud de correlación, el modelo teórico desarrollado puede predecir la conductividad térmica sin necesidad de otras medidas experimentales. Esto permitiría fabricar NWs con conductividades térmicas ad hoc. En efecto, el disponer de un modelo teórico que incorpora todos los parámetros que intervienen en la conductividad térmica, permite el diseño bajo demanda de las características físicas de los NWs para que estos tengan una conductividad térmica prefijada. Esta es una gran herramienta a la hora de diseñar dispositivos nanométricos con fines termoeléctricos. Por otro lado, el modelo desarrollado también es capaz de trabajar con NWs de aleaciones como el silicio-germanio. Estos NWs tienen una conductividad aún más baja que los NWs de silicio, y para algunas tareas pueden ser mucho más adecuados. Por tanto, disponer de un modelo teórico capaz de predecir cual es la conductividad térmica de estas estructuras resulta de gran ayuda a la hora de la gestión térmica de los nanodispositivos, sin tener que esperar a que existan medidas experimentales para el NW específico utilizado en cada nanodispositivo.

Gracias al estudio realizado en el capítulo 2 para conocer la conductividad térmica de los NWs de silicio y silicio-germanio, se ha podido modelizar en el capítulo 3 por elementos finitos la interacción térmica entre el láser y los NWs desde el punto de vista térmico, mediante la resolución de la ecuación del calor. Entender está interacción es un paso fundamental para poder realizar un correcto análisis de la señal obtenida al caracterizar estos NWs mediante métodos que empelan haces láser como fuente de excitación, en esta memoria el análisis se ha centrado en la espectroscopia micro-Raman. El análisis teórico nos muestra la enorme influencia 
que tiene el hecho de que las dimensiones radiales del NW se encuentren por debajo de la longitud de onda de la luz incidente, así como que también estén por debajo del tamaño efectivo del haz láser. En diversos trabajos científicos, el área de iluminación se describe a través de la densidad de potencia. Sin embargo, se ha mostrado como la distribución gaussiana de la intensad del haz láser provoca que varios NWs simultáneamente iluminados, no estén siendo excitados en las mismas condiciones. Esto, unido a la influencia de las dimensiones de cada NW, y de las características superficiales que modifican su conductividad térmica, hacen que al iluminar simultáneamente diferentes NWs, las temperaturas que estos alcanzan por efecto de la absorción de la luz puedan ser muy diferentes. De este modo, la respuesta Raman de cada NW iluminado puede ser muy diferente a la de los otros NWs simultáneamente iluminados, introduciendo una gran incertidumbre en la señal Raman recogida, e invalidando la interpretación dada a través de densidades de potencia homogéneas en la muestra. Con los datos procedentes del modelo teórico para la interacción térmica láser-NW, se ha caracterizado la incertidumbre introducida por las diferentes condiciones de excitación, simulando para ello los espectros Raman resultantes de diferentes configuraciones de NWs dentro del área de iluminación. De esta manera, se ha demostrado como al realizar una caracterización Raman sobre conjuntos de NWs, tanto los efectos de confinamiento fonónico, como los efectos de temperatura, pueden ser malinterpretados, e incluso confundidos, demostrando teóricamente que analizar conjuntos de NWs por espectroscopia Raman no es un procedimiento de caracterización fiable. En el capítulo 4, se ha demostrado experimentalmente como los espectros Raman tomados en conjuntos de NWs de silicio presentan muchas anomalías que dificultan su caracterización. El efecto de la temperatura se ha demostrado al iluminar una muestra de dos NWs paralelos y distinguir perfectamente como el espectro resultante es una contribución mezclada de ellos; por tanto incluso en muestras compuestas por pocos NWs el espectro Raman encierra una gran incertidumbre. Una vez establecido que la caracterización Raman debe ser realizada en NWs individuales, se han mostrado los efectos de la temperatura en los espectros Raman adquiridos en NWs individuales. El efecto de temperatura es muy difícil de eliminar en NWs que no estén depositados en substratos con buena disipación térmica, y ni aún así puede garantizarse que no aparezcan efectos de temperatura incluso a bajas potencias del láser. Para 


\section{RESUMEN Y CONCLUSIONES}

asegurar que se elimina cualquier efecto debido al calentamiento de los NWs, se ha establecido un método experimental consistente en analizar los espectros tomados en una linea de puntos de adquisición transversal al NW y que lo cruza por completo. Cuando no se aprecia variación en la posición del pico Raman en los espectros adquiridos en esa linea transversal, puede garantizarse que esa potencia láser no introduce ningún efecto apreciable sobre la temperatura del NW .

Una vez que podemos garantizar que no se induce un calentamiento en los NWs, se pueden realizar análisis espectrales complejos, como son los consistentes en determinar la composición de NWs de silicio-germanio a través de las posiciones de los picos Raman. La espectroscopia Raman ha demostrado ser una herramienta muy potente para el análisis composicional en estos NWs, arrojando valores compatibles con los resultados derivados de técnicas de caracterización más laboriosas como es el TEM+EDX.

Durante el análisis de los espectros Raman de los NWs de Silicio y silicio-germanio han aparecido diversos efectos que no están presentes en los espectros Raman de los materiales volúmicos. En la mayoría de los NWs, tanto de silicio como de siliciogermanio, aparece un pico suplementario a $495 \mathrm{~cm}^{-1}$. Diversos autores atribuyen la presencia de este pico a la presencia masiva de politipos de silicio wurtzita en los NWs; sin embargo, en nuestros hilos se ha demostrado que esta interpretación no se ajusta a lo observado. Este pico tiene una dependencia similar con la temperatura de los NWs que el pico Raman del silicio, por lo que se infiere que procede de enlaces Si-Si. Por otro lado, su frecuencia no está afectada por la presencia del germanio en la aleación; por tanto no procede de enlaces Si-Si del interior del NW puesto que su frecuencia estaría desplazada por el efecto de la aleación. Por otro lado su intensidad decae en los NWs de silicio-germanio cuanto mayor es la componente de germanio segregado en la superficie de los NWs. Esto nos indica que su procedencia está relacionada con algún tipo de efecto de superficie en los NWs. Otro efecto anómalo observado en los espectros Raman adquiridos en los NWs concierne a la intensidad Raman de los mismos. El volumen de los NWs es muy reducido en comparación con el volumen excitado en una muestra volúmica; sin embargo, y pese a esta enorme diferencia de volumen, las intensidades observadas en algunos NWs pueden ser incluso mayores que las medidas para el silicio 
volúmico de referencia. Finalmente, se ha observado que al realizar el análisis composicional de los NWs de silicio-germanio a través de las posiciones de los picos Ge-Ge y Si-Si se obtienen resultados muy diferentes. Mientras que la composición determinada a través del pico Si-Si se corresponde perfectamente con la composición determinada por EDX, la composición arrojada por el pico Ge-Ge muestra una composición falseada. Esto se debe a que los espectros obtenidos en estos NWs pueden descomponerse en dos contribuciones, una correspondiente a la aleación y otra correspondiente a una fase muy rica en germanio. Se ha demostrado que este espectro resultante es muy similar al obtenido en NWs çore-shellçon una cantidad de germanio muchísimo más elevada que la presente en nuestros NWs. Además de la anómala intensidad con la que se observa la fase correspondiente al germanio segregado, ésta depende de la posición que ocupa el NW dentro del área de iluminación del láser.

Para responder a estas anomalías observadas, en el capitulo 5 se ha modelizado por elementos finitos la interacción electromagnética existente entre el láser, el NW y el substrato donde se deposita. Mediante el cálculo de la distribución y valor de $|E|^{2}$ en el interior de los NWs, se ha demostrado que la enorme intensidad Raman observada en los NWs está relacionada con un acoplamiento electromagnético dependiente del diámetro de los NWs, la longitud de onda del haz láser y sobre todo de las características del substrato. Las resonancias introducidas por el substrato pueden aumentar la intensidad Raman procedente de los NWs en varios órdenes de magnitud. Este efecto abre la puerta a la sintonización de NWs-substratos, de tal modo que se pueda obtener una señal mensurable de NWs fabricados con materiales de muy baja señal Raman.

Por otro lado, se ha mostrado el efecto que tiene la orientación de los NWs respecto del eje de polarización del láser incidente cuando éste está linealmente polarizado. Se ha mostrado como la intensidad Raman es muy dependiente de la orientación del NW respecto al eje de polarización, pudiendo llegar a prácticamente extinguirse cuando ambos son perpendiculares. A través del modelo teórico, se ha demostrado como esta dependencia se debe principalmente al campo eléctrico en el interior de los NWs para cada orientación. El patrón de intensidades Raman con respecto al ángulo formado por el NW y eje de polarización del láser, se corresponde perfectamente con el patrón calculado teóricamente para $|E|^{2}$ tanto en NWs de silicio 


\section{RESUMEN Y CONCLUSIONES}

como de silicio-germanio, salvo cuando no se ilumina con una longitud de $532 \mathrm{~nm}$ los NWs de silicio. Además, para excitación con $532 \mathrm{~nm}$ existe otro efecto, que contribuye a la intensidad Raman y también parece depender de la orientación del NW. Por otra parte, se ha mostrado teóricamente que el patrón de intensidades Raman en función de su orientación, es un efecto acoplado en el que participan el diámetro del NW, la longitud de onda incidente y el substrato sobre el que se apoyan los NWs.

Por último, a través del estudio de la distribución de $|E|^{2}$ en el interior de los NWs, se ha demostrado como la intensidad Raman no procede de todo el volumen del NW, sino que para los diámetros analizados experimentalmente la distribución de $|E|^{2}$ está muy localizada en las zonas cercanas a la superficie de los NWs. Por tanto, la intensidad Raman procede principalmente de estas zonas mientras que el interior del NW apenas contribuye a la señal Raman final. De este modo puede explicarse que se observe de forma tan intensa el pequeño volumen correspondiente a la fase Ge segregada en NWs de silicio-germanio. Además, que $|E|^{2}$ esté tan concentrado en la superficie, nos indica que la presencia tan intensa del pico 495 se debe a algún efecto localizado en esa zona; téngase en cuenta que no todos los NWs presentan ese pico. Finalmente, se ha demostrado como el hecho de que $|E|^{2}$ este tan localizado en los NWs, justifica el efecto observado al variar la posición que el NW ocupa en el área de iluminación. $|E|^{2}$ se concentra en diferentes zonas de la superficie del NW en función de la posición que este ocupa en el área de iluminación; por tanto, como la distribución de germanio en superficie no es homogénea y como la señal procede de zonas muy localizadas, el espectro resultante muestra diferentes intensidades para la fase de germanio segregado.

Los resultados aquí presentados evidencian que cada hilo debe ser caracterizado individualmente, que la comprensión del comportamiento de los hilos individuales es una etapa necesaria para el diseño y fabricación de estructuras de nanohilos con propiedades orientadas a aplicaciones prediseñadas. Por otra parte, se ha demostrado que las propiedades ópticas de los nanohilos dependen de sus dimensiones, pero también de como se disponen, e.g. orientación, substrato...; en este sentido, podemos sugerir que se puede optimizar notablemente la colección de fotones (photon harvesting) mediante una adecuada selección de diámetros y substratos, lo que abre 
la puerta a mejoras en la eficiencia fotovoltaica, termoeléctrica, ó en fotosíntesis artificial. La interacción nanohilo/ luz es un campo que merece ser explorado en profundidad. 
6. RESUMEN Y CONCLUSIONES 


\title{
Apéndices
}

\subsection{Apéndice 1: Codigo Mathematica para el cálculo} de la conductividad térmica en NWs de silicio, germanio y silicio-germanio

\section{Conductividad térmica en NWs de Si, Ge y Si-Ge lisos y rugosos}

\author{
NWs con diám etros de 30 a $130 \mathrm{~nm}$
}

Parametrosde cada material:

\section{Si-Parameters}

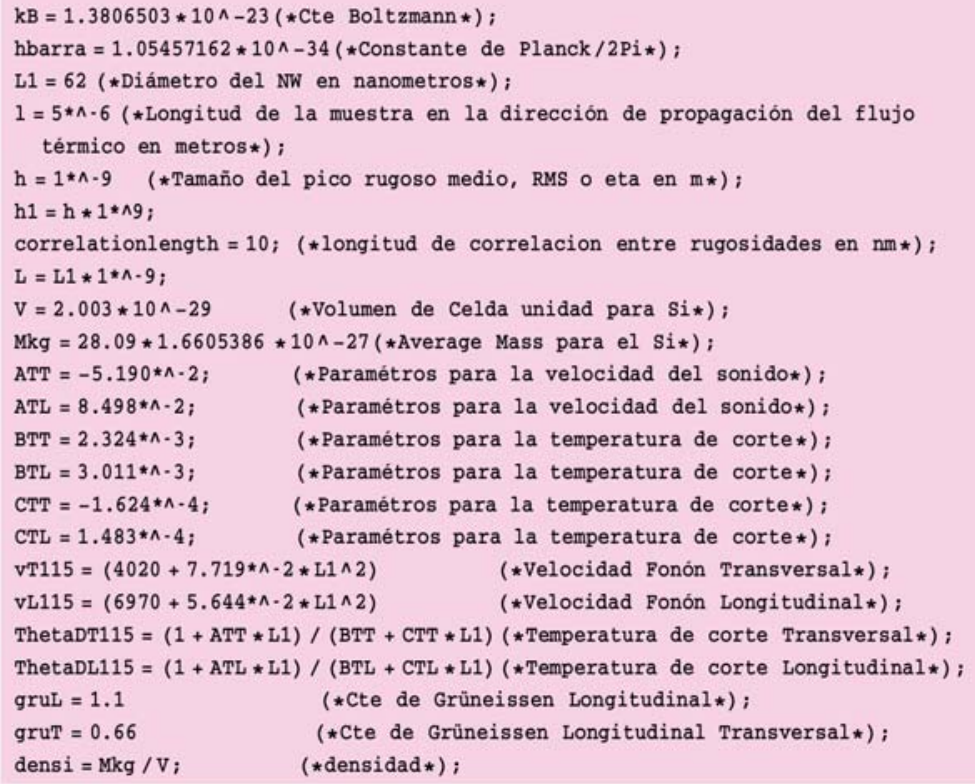




\subsection{Apéndice 1: Codigo Mathematica para el cálculo de la conductividad térmica en NWs de silicio, germanio y silicio-germanio}

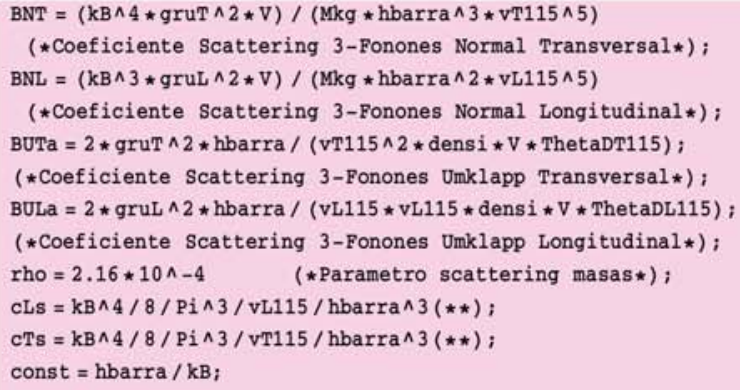

\section{Ge-Parameters}

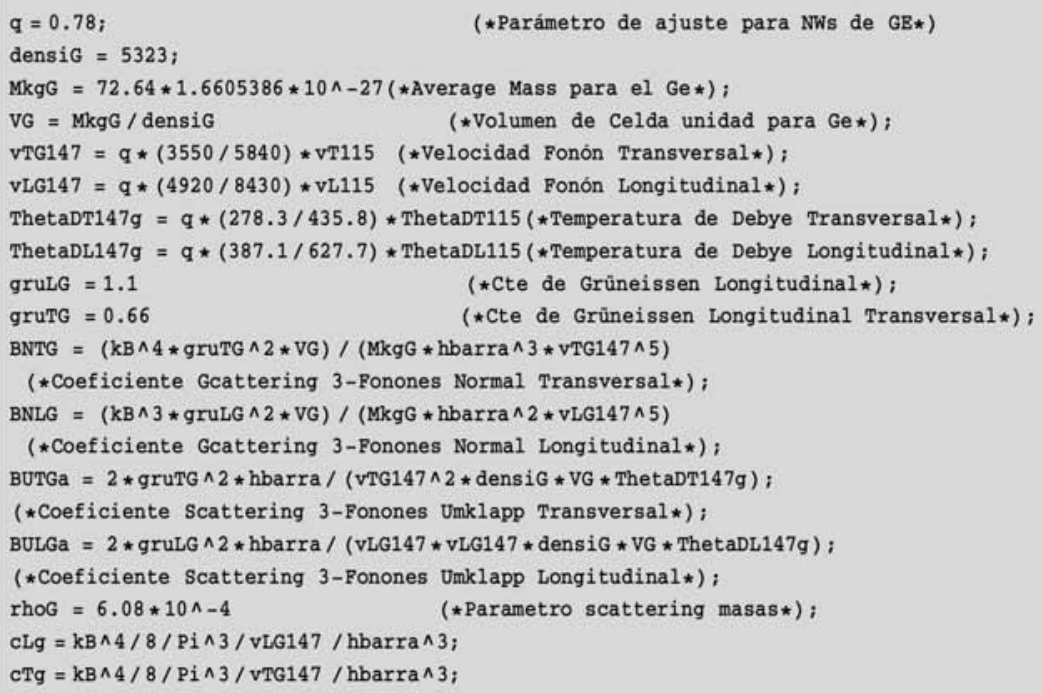

\section{SiGe-Parameters}

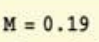

\section{Phonon-PointScattering}

tauLPP147S [Theta_, $\left.x_{-}, T_{-}\right]=1 /((\mathrm{V} *$ rho $* w[x, T] \wedge 4) /(4 * P i * \operatorname{vL} 115 \wedge 3))$;

tauTPP147S [Theta $\left., x_{-}, T_{-}\right]=1 /((V \star r h o \star w[x, T] \wedge 4) /(4 \star P i \star V T 115 \wedge 3))$

\section{Ge}

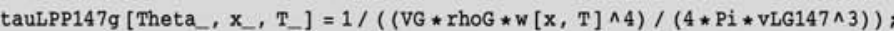

tauTPP147g [Theta $\left., x_{-}, T_{-}\right]=1 /(($ VG $\star \operatorname{rhoG} \star w[x, T] \wedge 4) /(4 \star P i \star V T G 147 \wedge 3))$, 


\section{APÉNDICES}

SiGe

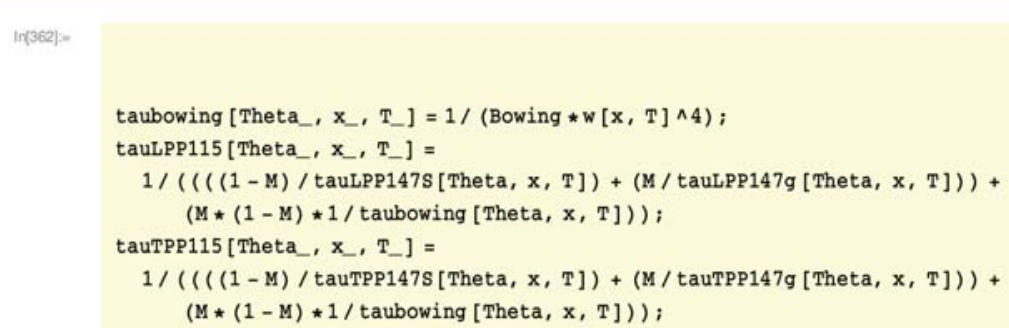

\section{Phonon-BoundaryScattering}

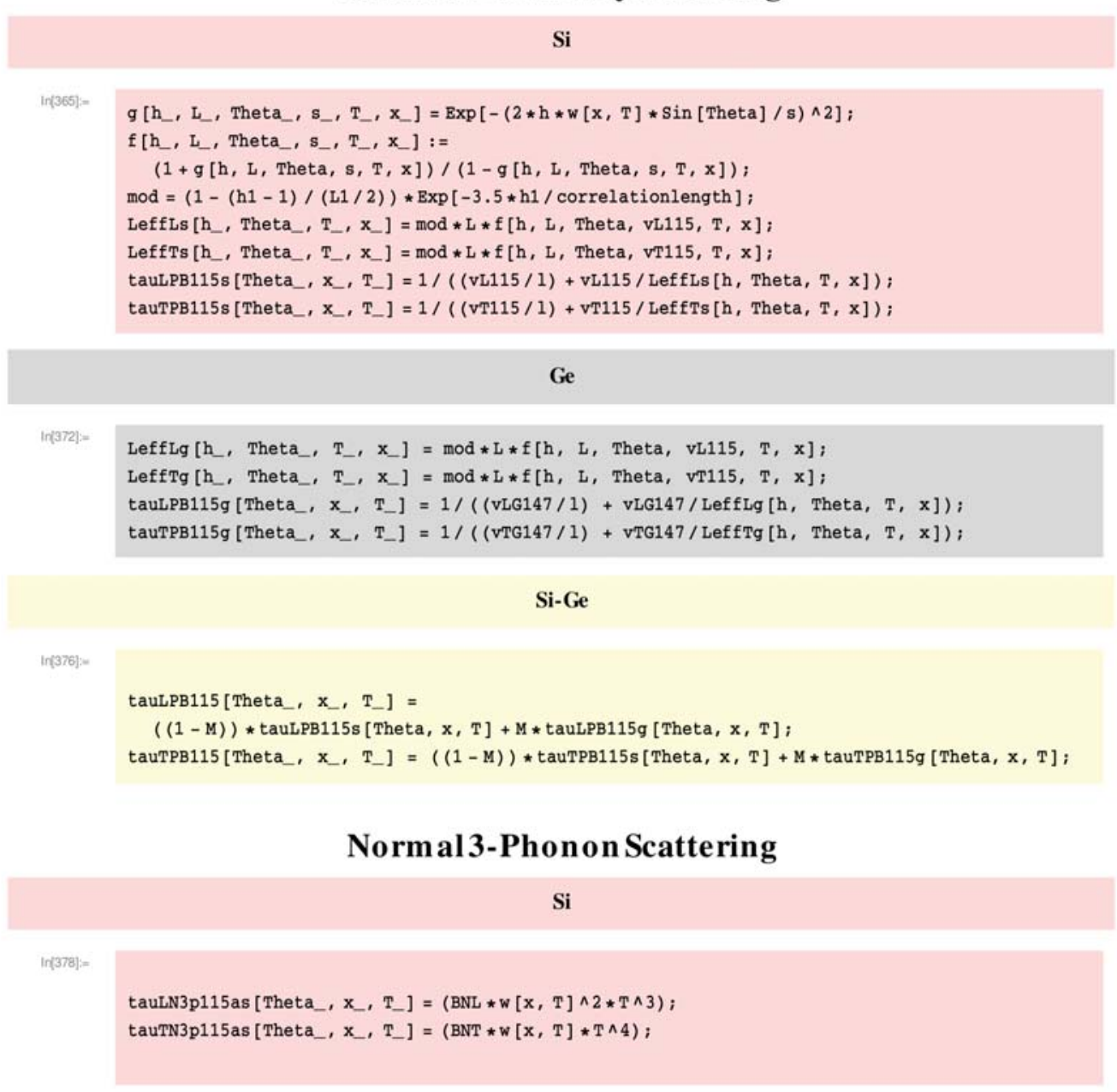


7.1 Apéndice 1: Codigo Mathematica para el cálculo de la conductividad térmica en NWs de silicio, germanio y silicio-germanio

4 / Modelo implementado en Mathematica para el calculo de la conductividad termica en NWs semiconductores.nb4

\begin{tabular}{|c|c|}
\hline \multicolumn{2}{|r|}{ Ge } \\
\hline $\ln [380]=$ & $\begin{array}{l}\left.\text { tauLN3p115ag [Theta_, } x_{-}, T_{-}\right]=(B N L G \star w[x, T] \wedge 2 \star T \wedge 3) ; \\
\text { tauTN3p115ag }\left[\text { Theta_, } x_{-}, T_{-}\right]=(B N T G \star w[x, T] \star T \wedge 4) ;\end{array}$ \\
\hline \multicolumn{2}{|r|}{ Si-Ge } \\
\hline $\operatorname{In}[3 \mathrm{Bg}\})=$ & $\begin{array}{l}\left.\text { tauLN3p115a [Theta_, } x_{-}, T_{-}\right]= \\
1 /((1-M) \star \text { tauLN3p115as }[\text { Theta, } x, T]+M \star \operatorname{tauLN} 3 p 115 a g[\text { Theta, } x, T]) ; \\
\left.\text { tauTN3p115a [Theta_, } x_{-}, T_{-}\right]= \\
1 /((1-M) \star \text { tauTN3p115as }[\text { Theta, } x, T]+M \star \operatorname{tauTN3p115ag~[Theta,~} x, T]) ;\end{array}$ \\
\hline
\end{tabular}

\section{Um klapp 3-Phonon Scattering}

\begin{tabular}{|c|c|}
\hline \multicolumn{2}{|r|}{$\mathrm{Si}$} \\
\hline $\ln (384)=$ & $\begin{array}{l}\text { tauLU3p115s }\left[\text { Theta }, x_{-}, T_{-}\right]=(B U L a * w[x, T] \wedge 2 \star T * \operatorname{Bxp}[-T h e t a D L 115 / 3 / T]) ; \\
\left.\operatorname{tauTU3p115s[Theta}, x_{-}, T_{-}\right]=(\text {BUTa } \star w[x, T] \wedge 2 \star T \star \operatorname{Exp}[-T h e t a D T 115 / 3 / T]) ;\end{array}$ \\
\hline \multicolumn{2}{|r|}{ Ge } \\
\hline $\ln \{386]:=$ & $\begin{array}{l}\left.\text { tauLU3p115g [Theta_, } x_{-}, T_{-}\right]=(\text {BULGa } * w[x, T] \wedge 2 \star T * \operatorname{Exp}[-T h e t a D L 147 g / 3 / T]) ; \\
\left.\text { tauTU3p115g[Theta_, } x_{-}, T_{-}\right]=(\text {BUTGa } * w[x, T] \wedge 2 \star T * \operatorname{Exp}[- \text { ThetaDT147g/3/T }]) ;\end{array}$ \\
\hline \multicolumn{2}{|r|}{ Si-Ge } \\
\hline $\ln (388\})=$ & 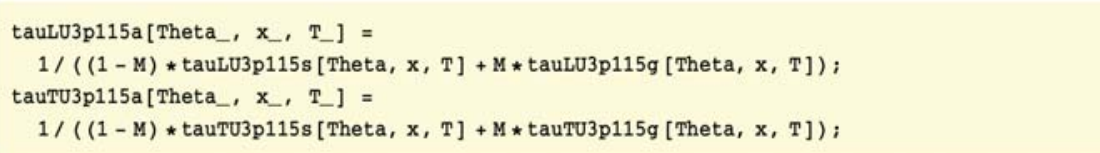 \\
\hline \multicolumn{2}{|r|}{ Cálculo de los tiem pos de relajación de Callaway } \\
\hline \multicolumn{2}{|l|}{ tau_R } \\
\hline $\ln (390)=$ & 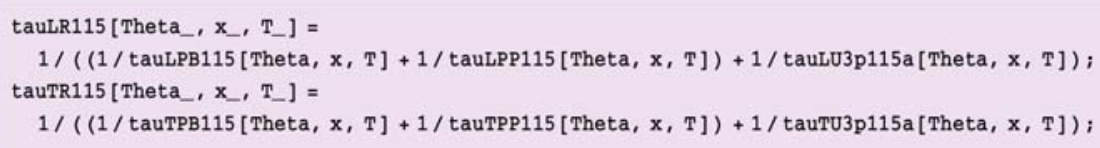 \\
\hline \multicolumn{2}{|l|}{ tau_C } \\
\hline $\ln (392) ;=$ & 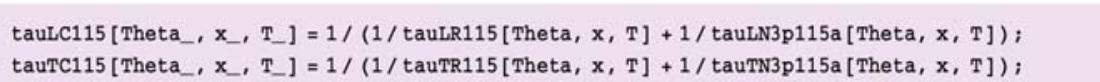 \\
\hline
\end{tabular}




\section{APÉNDICES}

\section{Integrandosde la ecuación 2.17}

\begin{tabular}{|c|c|}
\hline & Integando I1: \\
\hline $\operatorname{Ir}(394)]=$ & $\begin{array}{l}\text { I1L }\left[\text { Theta }, T_{-}, x_{-}\right]=\operatorname{Sin}[\text { Theta }] \star(\operatorname{Cos}[\text { Theta }]) \wedge 2 \star \operatorname{tauLC1} 15[\text { Theta, } x, T] * \\
\quad((x \wedge 4) \star \operatorname{Exp}[x] /(\operatorname{Exp}[x]-1) \wedge 2) ; \\
\text { I1T }\left[\text { Theta_, } T_{-}, x_{-}\right]=\operatorname{Sin}[\text { Theta }] \star(\operatorname{Cos}[\text { Theta }]) \wedge 2 \star \operatorname{tauTC} 115[\text { Theta, } x, T] * \\
\left(\left(x^{\wedge} 4\right) \star \operatorname{Exp}[x] /(\operatorname{Exp}[x]-1) \wedge 2\right) ;\end{array}$ \\
\hline
\end{tabular}

\section{Integando I2:}

$\ln (396) ;-$

I2L $\left[\right.$ Theta $\left., T_{-}, x_{-}\right]=\operatorname{Sin}[$ Theta $] *(\operatorname{Cos}[$ Theta $]) \wedge 2$ *

(taulc115[Theta, $x, T] / \operatorname{tauLN3p115a[Theta,~} x, T]) \star((x \wedge 4) \star \operatorname{Exp}[x] /(\operatorname{Exp}[x]-1) \wedge 2)$;

I2T $\left[\right.$ Theta $\left., T_{-}, x_{-}\right]=\operatorname{Sin}[$ Theta $] *(\operatorname{Cos}[$ Theta $]) \wedge 2 *$

$($ tautc115 $[$ Theta, $x, T] / \operatorname{tauTN} 3 p 115 a[$ Theta $, x, T]) \star((x \wedge 4) \star \operatorname{Exp}[x] /(\operatorname{Exp}[x]-1) \wedge 2)$;

\section{BETA:}

$\ln \{398)$ :

BETAsL [Theta $\left., T_{-}, x_{-}\right]=\operatorname{Sin}[$ Theta $] \star(\operatorname{Cos}[$ Theta $]) \wedge 2$ *

(taulc115[Theta, $x, T] /$ taulN3p115a [Theta, $x, T]) *((x \wedge 4) \star \operatorname{Exp}[x] /(\operatorname{Exp}[x]-1) \wedge 2) ;$

BETAsT [Theta, $\left.T_{-}, x_{-}\right]=\operatorname{Sin}[$ Theta] * $(\operatorname{Cos}[$ Theta $]) \wedge 2$ *

(tauTC115[Theta, $x, T] / \operatorname{tauTN3p115a}[$ Theta, $x, T]) \star((x \wedge 4) \star \operatorname{Exp}[x] /(\operatorname{Exp}[x]-1) \wedge 2)$;

BETAiL [Theta_, $\left.T_{-}, x_{-}\right]=\operatorname{Sin}[$ Theta $] *(\operatorname{Cos}[$ Theta $]) \wedge 2 *(1 /$ tauln $3 p 115 a[$ Theta, $x, T])$ *

$(1-\operatorname{tauLC1} 15[$ Theta, $x, T] /$ tauLN3p115a [Theta, $x, T]) \star((x \wedge 4) \star \operatorname{Exp}[x] /(\operatorname{Exp}[x]-1) \wedge 2)$;

BETAiT [Theta $\left., T_{-}, x_{-}\right]=\operatorname{Sin}[$ Theta] * $(\operatorname{Cos}[$ Theta $]) \wedge 2 *(1 /$ tauTN3p115a [Theta, $x, T])$ *

$(1-\operatorname{tauTc1} 15[$ Theta, $\mathrm{x}, \mathrm{T}] / \operatorname{tauTN} 3 \mathrm{p} 115 \mathrm{a}[$ Theta, $\mathrm{x}, \mathrm{T}]) \star((\mathrm{x} \wedge 4) \star \operatorname{Exp}[\mathrm{x}] /(\operatorname{Exp}[\mathrm{x}]-1) \wedge 2)$; 


\subsection{Apéndice 1: Codigo Mathematica para el cálculo de la conductividad térmica en NWs de silicio, germanio y silicio-germanio}

6 / Modelo implementado en Mathematica para el calculo de la conductividad termica en NWs semiconductores.nb6

\section{Cálculo de la conductividadtérmica porintegración númerica:}

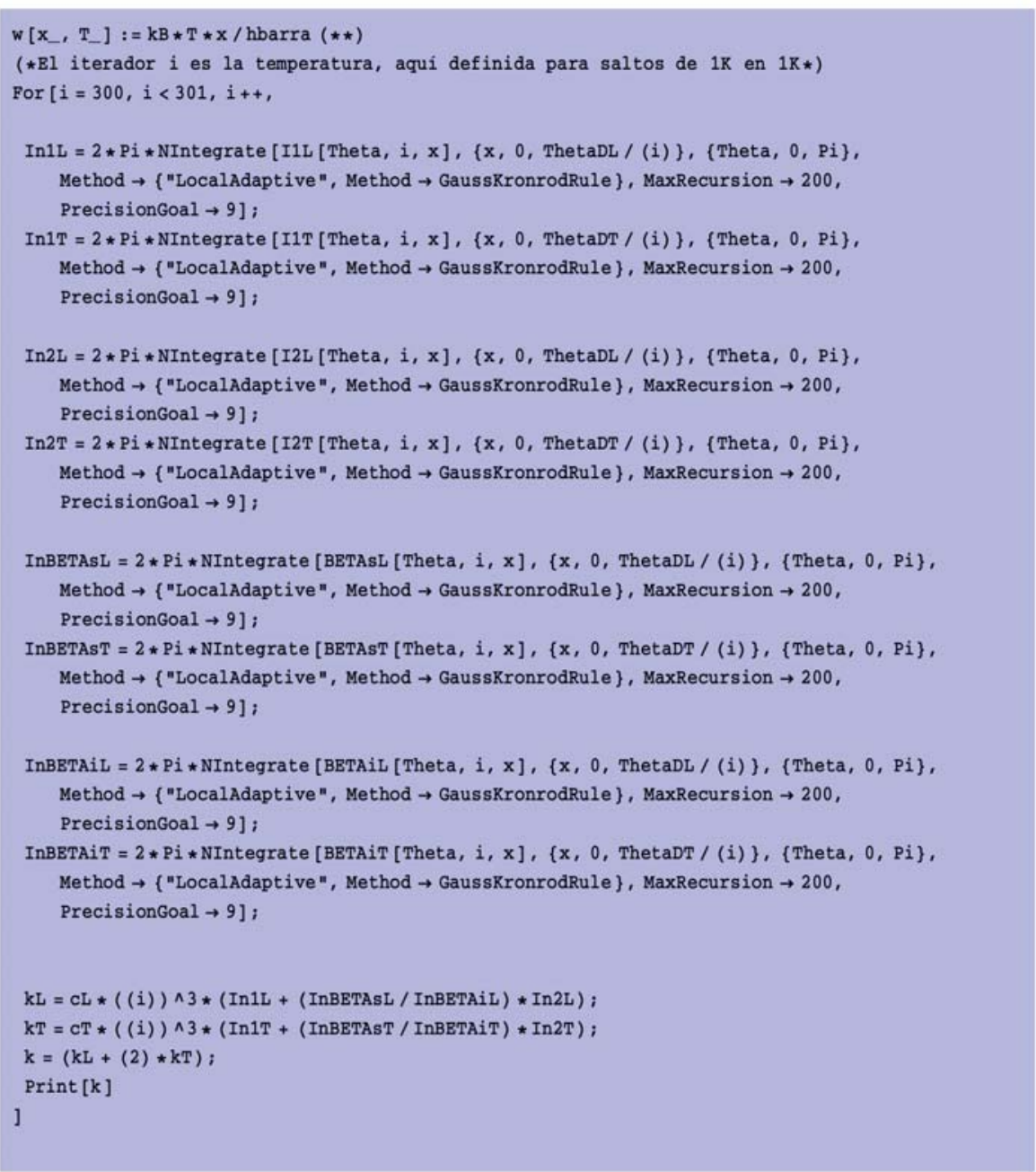




\subsection{Apendice 2: Publicaciones generadas durante la realización de este trabajo de investigación}

\section{Publicaciones en revistas}

"Raman spectroscopy study of group IV semiconductor nanowires"

J. Anaya, A. Torres, A. Martín-Martín, O. Martínez, A.C. Prieto, J. Jiménez, A. Rodríguez, J. Sangrador, T. Rodríguez

Physics Procedia 8, 78-83, 2010

"Si and SixGe1-x NWs studied by Raman spectroscopy"

J. Anaya, A. C. Prieto, O. Martínez, A. Torres, A. Martín-Martín, J. Jiménez, A. Rodríguez, J. Sangrador,T. Rodríguez

Phy. Status Solidi C, 8, 1307-1310, 2011

"MicroRaman Spectroscopy of Si Nanowires: Influence of Size"

J. Anaya, C. Prieto, A. Torres, A. Martín-Martín, J. Souto, J. Jiménez, A. Rodríguez, T. Rodríguez

Materials Science Forum, 725, 255, 2012

"Interaction Between a Laser Beam and Semiconductor Nanowires: Application to the Raman Spectrum of Si Nanowires"

J. Anaya, A. Torres, C. Prieto, J. Jimenez, A. Rodríguez, T. Rodríguez.

Int. J. of Nanoparticles. (in Press)

"Study of the temperature distribution in Si nanowires under microscopic laser beam excitation"

J. Anaya, A. Torres, A. Martín-Martín, J. Souto, J. Jiménez, A. Rodríguez, T. Rodríguez

App. Phys. A, DOI 10.1007/s00339-012-7509-y, 2012

"Defect Signatures in Degraded High Power Laser Diodes"

V. Hortelano, J. Anaya, J. Souto, J. Jimenez, J. Perinet, F. Laurelle. 
Pendiente de publicación en Microelectronics Reliability.

"Raman spectrum of Si nanowires: temperature and phonon confinement effects"

J. Anaya, A. Torres, J. Jiménez, A. Rodríguez, T. Rodríguez, L. Pichon

Pendiente de publicación en Applied Physics A.

\section{Capítulos en Libros}

"Nanowires - Recent Advances, Ch 11, Thermal Transport in Semiconductor Nanowires"

edited by Xihong Peng

J. Anaya, J. Jiménez, T. Rodríguez.

DOI: 10.5772/52588, ISBN 978-953-51-0898-6, 2012.

\section{Proceedings de Conferencias}

"Luminescence studies of $\mathrm{ZnO}$ nanowires for photovoltaic applications: effect of size diameter"

O. Martínez, V. Hortelano, J. Anaya, J. Jiménez, F. Güell, J.R. Morante.

Springer Proceedings in Physics, (accepted), 2011

"Thermal properties of group IV nanowires under laser beam excitation" J.Anaya, C.Prieto, J. Jiménez, A.Rodríguez, T.Rodríguez

Springer Proceedings in Physics, (accepted), 2011

"Raman spectrum of group IV nanowires: influence of temperature"

J. Anaya, C. Prieto, J. Souto, J. Jiménez, A. Rodríguez, J. Sangrador, T. Rodríguez MRS Proceedings, 1305, mrsf10-1305-aa12-03, 2011.

"Laser Induced Heating of Group IV Nanowires" J. Anaya, A. Torres, A. MartínMartín, J. Jiménez, A. Rodríguez and T. Rodríguez. 


\section{APÉNDICES}

MRS Proceedings, 1404, mrsf11-1404-w07-03, 2012.

"Thermomechanical modelling of high power laser diode degradation"

J. Anaya, A. Martin-Martin, J. Souto, P. Iñiguez and J. Jimenez.

MRS Proceedings, 1432, mrss12-1432-g01-05, 2012.

"Modelling the thermal conductivity of semiconductor NWs; A step forward to the increase of the thermoelectric figure of merit"

J. Anaya, J. Jiménez, T. Rodríguez.

IEEE proceedings of the 9th Spanish Conference on Electron Devices. (In press)

"Degradation signatures of high-power laser diodes"

J. Jiménez, J. Anaya, V. Hortelano, J. Souto, A. Martín.

IEEE proceedings of the 9th Spanish Conference on Electron Devices.

\section{Presentaciones en Conferencias}

"Raman spectroscopy study of group IV semiconductor nanowires"

J. Anaya, A. Torres, A. Martín-Martín, O. Martínez, A.C. Prieto, J. Jiménez, A. Rodríguez, J. Sangrador, T. Rodríguez

VI Encuentro Franco-Español de Química y Física del Estado Sólido Tarragona, Spain 17/03/2010

Poster

"Raman Spectrum of Group IV Nanowires: Influence of Temperature."

J. Anaya, C. Prieto, J. Souto, J. Jiménez, A. Rodríguez, J. Sangrador, T. Rodríguez 2010 MRS Fall Meeting

Boston, USA 29/11/ 2010

Oral communication (Prof. J.Jimenez)

"Luminescence studies of $\mathrm{ZnO}$ nanowires for photovoltaic applications: effect of size diameter" 
O. Martínez, V. Hortelano, J. Anaya, J. Jiménez, F. Güell, J.R. Morante.

E-MRS 2011 Spring \& Bilateral Meeting

Nice, France 09/05/ 2011 Poster

"Thermal properties of group IV nanowires under laser beam excitation"

J.Anaya, C.Prieto, J. Jiménez, A.Rodríguez, T.Rodríguez

E-MRS 2011 Spring \& Bilateral Meeting

Nice, France 09/05/ 2011 Oral communication (Prof. J.Jimenez)

"Influence of Temperature and Dimensions on the Micro-Raman Spectrum of Si Nanowires"

J. Anaya, A.C. Prieto, A. Torres, A. Martín-Martín, J. Jiménez, A. Rodríguez, J. Sangrador, T. Rodríguez

14th International Conference on Defects, Recognition, Imaging and Physics in Semiconductors DRIP XIV

Miyazaki, Japan 25/09/ 2011 Oral communication (Prof. J.Jimenez)

\section{"Laser Induced Heating of Group IV Nanowires"}

J. Anaya, A. Torres, A. Martín-Martín, J. Jiménez, A. Rodríguez and T. Rodríguez. 2011 MRS Fall Meeting \& Exhibit

Boston, USA 28/11/ 2011 Oral communication (Prof. J.Jimenez)

"Thermomechanical modelling of high power laser diode degradation"

J. Anaya, A. Martín-Martín, J. Souto, P. Iñiguez and J. Jiménez

2012 MRS Spring Meeting \& Exhibit

Boston, USA 09/04/ 2012 Oral communication (Prof. J.Jimenez)

"In-situ Raman analysis of illustrations of the "Paregon Atlas" (1603) and “Geographicae Enarratione, Libri Octo" (1525)"

Prieto, A. C, Martínez, O, Anaya, J, Jiménez, J, Mastres, M. L, Pastrana, P and Barrera, M

GEORAMAN 10 - Nancy - 11-13 June 2012 


\section{APÉNDICES}

Nancy, France 11/06/ 2012 Poster

"Interaction Between a Laser Beam and Semiconductor Nanowires: Application to the Raman Spectrum of Si Nanowires"

J. Anaya, A. Torres, C. Prieto, J. Jimenez, A. Rodríguez, T. Rodríguez.

11th International Workshop on Beam Injection Assessment of Microstructures (BIAMS 11)

Annaba, Algeria 25/06/ 2012 Invited Oral communication (Prof. J.Jimenez)

"Role of thermal conductivity in the degradation of high power laser diodes" J. Anaya, A. Martín-Martín, J. Souto, P. Iñiguez and J. Jiménez International Symposium on Reliability of Optoelectronics for Space (ISROS 2012) Cagliari, Italy 01/05/ 2012 Oral communication (Prof. J.Jimenez)

"SiGe Nanowires, Structural and MicroRaman Spectroscopy Characterization" J.Anaya, J. Jimenez, A. Rodríguez, T. Rodríguez 2012 MRS Fall Meeting, Boston, USA. 25/11/ 2012 Oral communication (Prof. J.Jimenez)

"Degradation signatures of high-power laser diodes"

J. Jiménez, J. Anaya, V. Hortelano, J. Souto, A. Martín.

9th Spanish Conference on Electron Devices.

Valladolid, Spain 12/02/ 2013 Invited Oral communication (Prof. J.Jimenez)

"Modelling the thermal conductivity of semiconductor NWs; A step forward to the increase of the thermoelectric figure of merit"

J. Anaya, J. Jiménez, T. Rodríguez.

9th Spanish Conference on Electron Devices.

Valladolid, Spain 12/02/ 2013 Poster

\section{Publicaciones en preparación}

"Predictive model for the calculus of the thermal conductivity of Si and Si(1-x)Gex NWs” J. Anaya, J. Jiménez, T. Rodríguez. 
7.2 Apendice 2: Publicaciones generadas durante la realización de este trabajo de investigación

"Raman spectrum of Si and Si(1-x)Gex nanowires"

J. Anaya, A. Torres, J. Jiménez, A. Rodríguez, T. Rodríguez, L. Pichon 


\section{Declaración de originalidad}

Por la presente declaro que he producido esta obra sin recurrir a plagio o aprovechando el trabajo de terceras personas sin reconocimiento explicito, habiendo sido identificadas claramente las fuentes de los resultados externos utilizados en este documento.

Este trabajo no ha sido previamente presentado de manera idéntica o similar a cualquier tribunal examinador.

Este trabajo ha sido desarrollado entre el 2011 y el 2013 bajo la atenta supervisión del profesor Juan Jiménez López en el grupo de semiconductores OptronLab de la Universidad de Valladolid.

Valladolid,

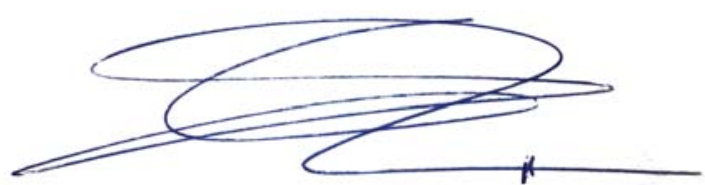




. 